This item was submitted to Loughborough's Research Repository by the author.

Items in Figshare are protected by copyright, with all rights reserved, unless otherwise indicated.

\title{
Ultra-sensitive carbon based molecular sensors
}

PLEASE CITE THE PUBLISHED VERSION

\author{
PUBLISHER \\ (C) Jingfeng Huang

\section{PUBLISHER STATEMENT}

This work is made available according to the conditions of the Creative Commons Attribution-NonCommercialNoDerivatives 4.0 International (CC BY-NC-ND 4.0) licence. Full details of this licence are available at: https://creativecommons.org/licenses/by-nc-nd/4.0/

\section{LICENCE}

CC BY-NC-ND 4.0

\section{REPOSITORY RECORD}

Huang, Jingfeng. 2019. "Ultra-sensitive Carbon Based Molecular Sensors". figshare. https://hdl.handle.net/2134/17963. 


\section{ULTRA-SENSITIVE CARBON BASED \\ MOLECULAR SENSORS}

HUANG JINGFENG

SCHOOL OF MATERIALS SCIENCE AND

ENGINEERING, NTU

SCHOOL OF SPORT, EXERCISE AND HEALTH

SCIENCES, LU

2014 


\title{
ULTRA-SENSITIVE CARBON BASED
}

\author{
MOLECULAR SENSORS
}

\section{HUANG JINGFENG}

\author{
A thesis submitted to the \\ Nanyang Technological University \\ (School of Materials Science and Engineering) \\ $\&$

\section{Loughborough University} \\ (School of Sport, Exercise and Health Sciences) \\ in fulfilment of the requirement for the Joint degree of \\ Doctor of Philosophy
}




\section{$\underline{\text { PROLOGUe }}$}

${ }^{66}$ Carbon is only the fifteenth most common element, accounting for a very modest 0.048 percent of the Earth's crust, but we would be lost without it. What sets the carbon atom apart is that it is shamelessly promiscuous. It is the party animal of the atomic world, latching on to many other atoms (including itself) and holding tight, forming molecular conga lines of hearty robustness... As Paul Davies $^{1}$ has written: If it wasn't for carbon, life as we know it would be impossible.", 2

${ }^{1}$ Paul Davies, The Origin of Life, Penguin UK, 2003, p. 145.

${ }^{2}$ Bill Bryson, A Short History of Nearly Everything, Black Swan, Random House, 2004, p. 309. 


\section{AbSTRACT}

This thesis presented the study of carbon-based materials for ultra-sensitive molecular sensing.

Reduced Graphene Oxide (rGO), a 2-dimensional one-atomic layer thick carbon material, had the advantage of low-cost, aqueous and industrial-scalable production route. Using rGO as the transducer platform could potentially lower the cost of sensors down to a few dollars per chip. However, there were still limitations in rGO that prevented its widespread usage as a biosensor transducer or in electronics: its low electrical conductivity and large electrical deviations. This thesis was structured to understand and solve these problems for transducer application. The thesis could be broken down into 3 parts:

The first part of the thesis presented the critical review of the background and limitations of graphene research, followed by the background and importance of biosensor developments for the detection of sweat sodium ions and circulatory Interleukin-6 proteins.

The second part of the thesis tested the hypothesis that the rGO limitations could be eliminated to create a highly sensitive biosensor transducer via (A) improving rGO synthesis (B) pristine Carbon Nanotubes-rGO hybrid film and (C) growth of rGO. The mechanism of ultra-large graphene oxide synthesis and graphene oxide growth was also elucidated in this section.

The third part of the thesis then presented the fabrication and test of the practical and homogenous carbon-based biosensor using the transducer synthesized earlier. The thesis showed that through proving the hypothesis correct, it enabled the synthesis of an all organic sodium ion sensor with integrated pump and an ultra-sensitive interleukin-6 bio-sensor. Both of these novel sensors were able to detect the respective molecules in their physiological ranges.

Keywords: Reduced Graphene Oxide; Chemically Modified Graphene; Biosensors; Interleukin-6; Sodium. 


\section{ACKNOWLEDGEMENTS}

My acknowledgements and appreciation go to the following people, whose contributions and support helped made the completion of this thesis possible.

Prof. Alfred Tok I.Y. and Prof. Myra A. Nimmo,

for their continuous supervision, time, patience, support, guidance regarding many aspects of my professional development. This thesis would not be possible without both.

Prof. Zhang Hua, Dr. Christoph Nowak, Dr. Melanie Larisika,

Dr. Mark Platt, Dr. Steve Faulkner, Dr. Derrick Fam,

for their mentorship, candid feedback and answering numerous questions during experiments.

Groups, staffs, peers, friends at Nanyang Technological University and

Loughborough University in School of Sport, Exercise and Health Sciences

especially Andres Herrera, Zou Changji, James Harvey, Chen Hu,

for their invaluable advice, encouragement and being great colleagues to work with.

My family,

for believing in me and were always there.

Finally, I would like to acknowledge the Institute for Sports Research of Nanyang Technological University (NTU); School of Sport, Exercise and

Health Sciences of Loughborough University; Austrian Institute of Technology; Centre for Biomimetic Sensor Science of NTU; Singapore Economic Development Board and NTU Research Scholarship for funding.

The author hopes to dedicate this work to the betterment of humanity through technological breakthroughs. 


\section{$\underline{\text { TABLE OF CONTENTS }}$}

Prologue I

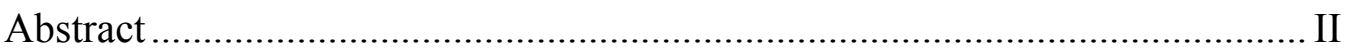

Acknowledgements ...................................................................................II

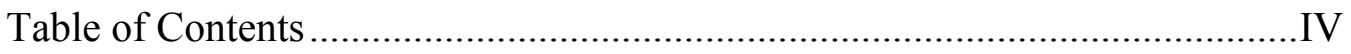

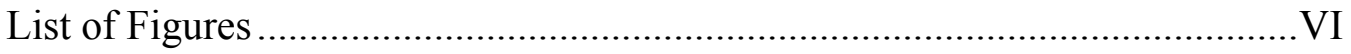

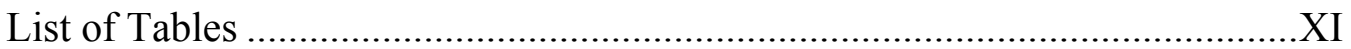

Chapter 1 Literature Review ....................................................................... 1

1.1 Overview of Graphene and Graphene Oxide................................................. 1

1.2 Structure and Properties of Graphene Oxide ......................................................... 3

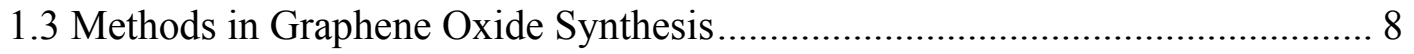

1.4 Structure \& Properties of Reduced Graphene Oxide ......................................... 19

1.5 Methods in Reduced Graphene Oxide Synthesis ........................................... 21

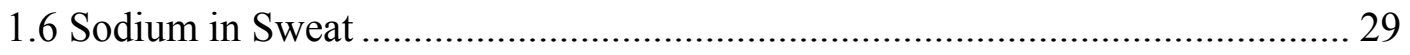

1.7 Interleukin-6 in Circulation and in Sweat .................................................... 35

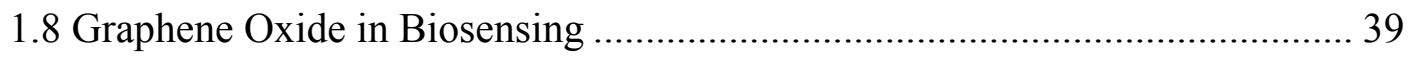

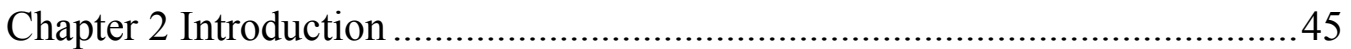

2.1 Current Challenges and Motivations .......................................................... 45

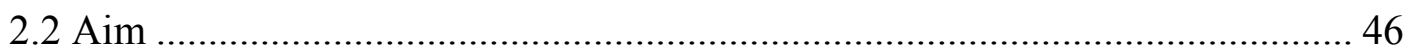

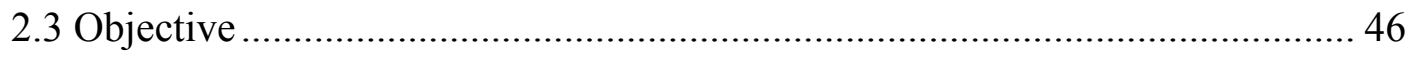

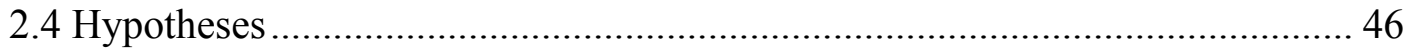

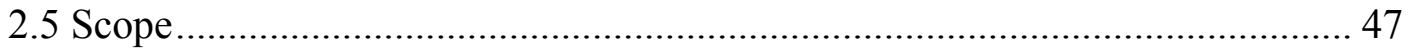

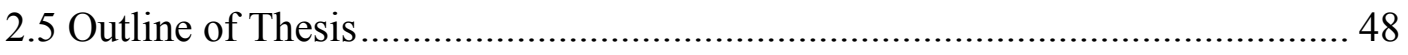

Chapter 3: Preparation and Mechanism of Ultra-Large Graphene Oxide Flakes

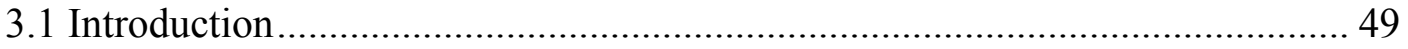

3.2 Improved Procedure for Large GO Flakes................................................... 51

3.3 Mechanism of Large GO Flakes …............................................................. 52

3.4 Characterization of Large GO Flakes ....................................................... 58

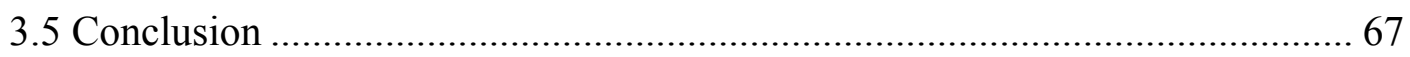

Chapter 4: Development of a Disposable, All Organic Biosensor with Integrated Pump for Sodium Detection .68

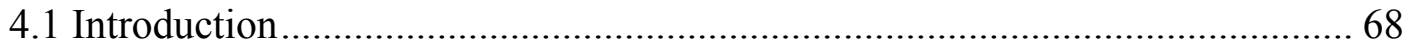

4.2 Idea of Fully Organic Sodium Sensor............................................................ 69 
4.3 Integrating Sweat Collection

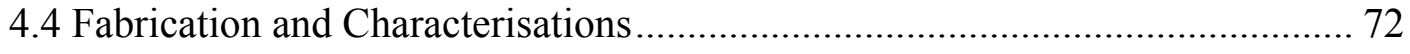

4.5 Pump Testing and Sodium Sensing ............................................................. 75

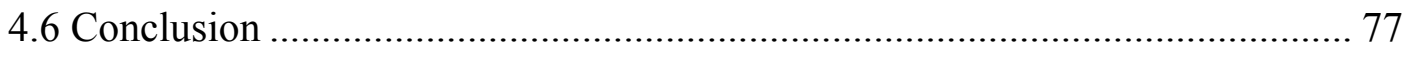

Chapter 5: Novel Carbon Nanotube-Graphene Oxide Hybrid Structure and its Mechanism .78

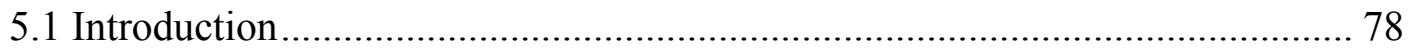

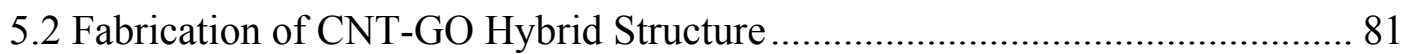

5.3 Characterization and Discussion of CNT-GO Hybrid Structure ..................... 82

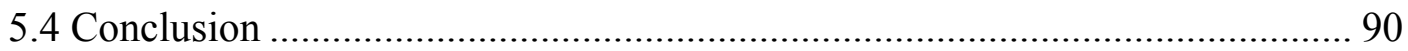

Chapter 6: Reduced Graphene Oxide Growth and its Mechanism ...................91

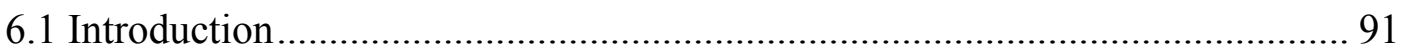

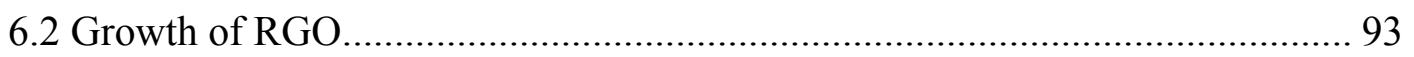

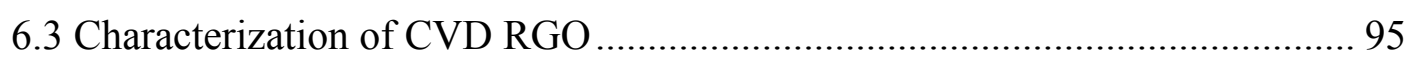

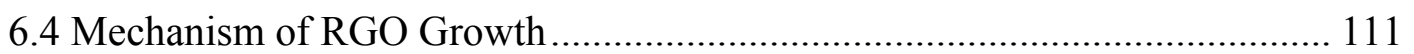

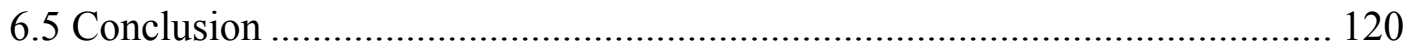

Chapter 7: Development of Novel Biosensor for IL-6 Detection and Determining its Presence in Sweat

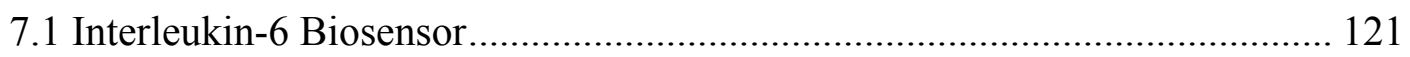

7.2 Ethanol CVD rGO Transducer Fabrication .................................................. 123

7.3 IL-6 Biosensor Characterization and Test .................................................... 124

7.4 Presence of IL-6 in Sweat ............................................................................ 129

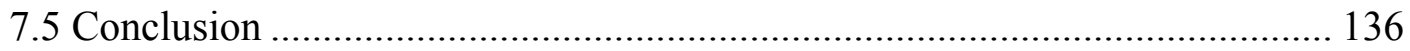

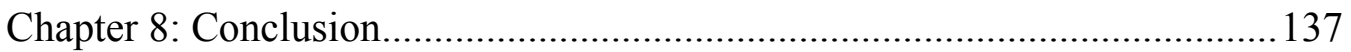

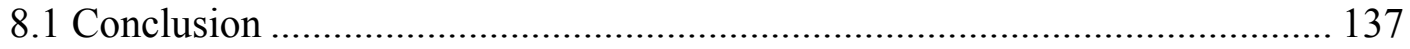

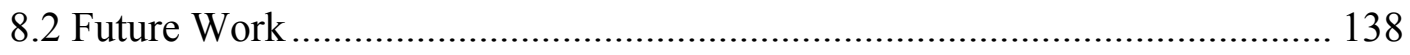

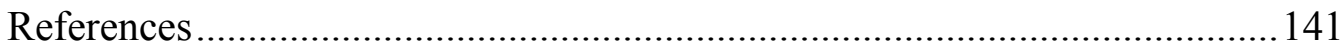

Annex: List of Scientific Publications and Achievements ............................. 163

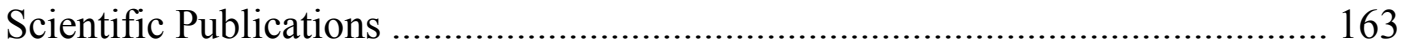

International Conferences and Workshops ...................................................... 166

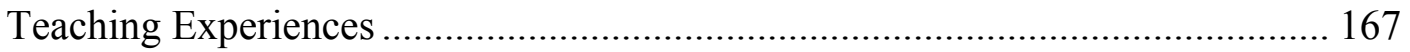

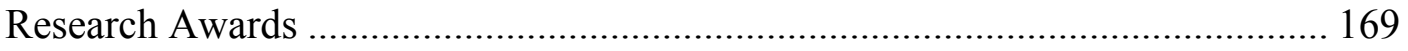

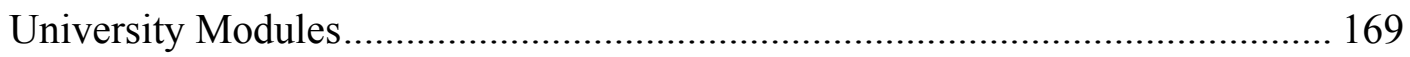




\section{LIST OF FIGURES}

Figure 1: Number of publications on carbon-based materials over past 20 years.

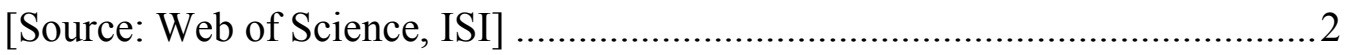

Figure 2: Structural model of GO proposed by Lerf et al. ${ }^{30}$............................... 4

Figure 3: (a) Surface species of GO proposed by Szabo with the inclusion of $(\mathrm{C}=\mathrm{O})$ in basal planes (b) Boundaries between domains of $\mathrm{GO}$ causes folded carbon skeletons ${ }^{36}$

Figure 4: Aberration-corrected TEM image of GO, with scale bar 2nm, showing that $\mathrm{sp}^{2}$ islands were isolated in a $\mathrm{sp}^{3}$ matrix. Magnified images of (a) oxidized $\mathrm{sp} 3$ (b) possible mobile oxygen functionalities (c) graphitic $\mathrm{sp}^{2}$ of $\mathrm{GO}^{46}$..........6 6

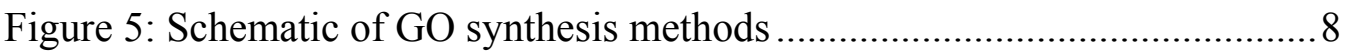
Figure 6: Schematic showing the anodic oxidation, intercalation and exfoliation steps to produce single and multilayer $\mathrm{GO}^{71}$.................................................. 11 Figure 7: Number of publications on modified Hummer's method over past 20 years. [Source: Web of Science, ISI]

Figure 8: Possible oxygen functional groups and carbon arrangements formed after annealing (a) pair of carbonyls, (b) carbon chain, (c) pyran, (d) furan, (e) pyrone, (f) 1,2-quinone, (g) 1,4-quinone (h) five-member carbon ring (i) threemember carbon ring $(j)$ phenol. The darkest shade was oxygen, followed by carbon and no shading in hydrogen atoms. Adapted from ref. ${ }^{93}$

Figure 9: Conductivity of thermally reduced GO as a function of $\mathrm{sp} 2$ fraction from XPS. Experimental data fitting reveals two different regimes for electrical transport. Tunneling/hopping dominate transport at $\mathrm{sp}^{2}$ fractions below 0.6 while percolation dominated above. Three references were given as comparison; values of graphene with minimum conductivity (lower triangle), doped conductivity of ideal graphene (upper triangle) and polycrystalline graphite (square). Insert shows evolution of GO structural model at different stage of reduction. Darker areas represent $\mathrm{sp}^{2}$ clusters and lighter areas represents $\mathrm{sp}^{3}$

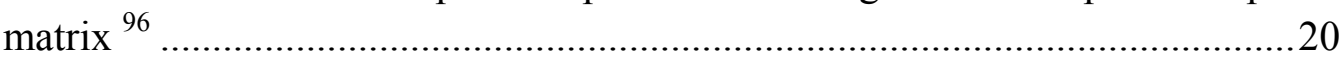

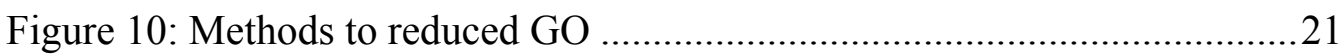

Figure 11: A proposed reaction pathway for epoxide reduction with hydrazine ${ }^{40}$

Figure 12: Various salts' ion concentration vs. sweat rates. With increasing sweat rate, $\mathrm{Na}^{+}$and $\mathrm{Cl}^{-}$increased while the other electrolytes decreased or remained the same ${ }^{144}$

Figure 13: Regression of 5-site and individual RSC sweat $\mathrm{Na}^{+}$concentration. The dashed line was the line of identity. It was observed that the thigh sweat RSC correlated well with WBW value ${ }^{143}$ 
Figure 14: Sweat sodium concentration versus back sweat rate during exercise in heat between untrained and trained groups. Higher aerobic fitness level increased both sweat rate and $\mathrm{Na}^{+}$concentration in sweat ${ }^{155}$

Figure 15: $\mathrm{Na}^{+}$concentration in sweat vs. sweat rate on Day 1 and Day 10 of heat acclimation ${ }^{140}$

Figure 16: Representation of the JAK/STAT (Janus kinase/Signal transducers and activators of transcription) pathway and the MAPK (mitogen-activated protein kinase) cascade by IL- $6^{169}$

Figure 17: Schematic presentation showing effect of strenuous exercise on the levels of interleukin (IL)-6, interleukin-1 receptor antagonist (IL-1ra), macrophage inflammatory protein (MIP-1 $\beta$ ), IL-8, soluble tumour necrosis factor- $\alpha$ receptor (sTNF- $\alpha \mathrm{R}$ ), TNF- $\alpha$, and IL-1. The relative increase in plasma concentrations of cytokines during and after exercise was shown ${ }^{174}$

Figure 18: Increase of IL-6 in plasma and urine after running. Both samples were collected before exercise, directly after finishing ( 2 hours) and at indicated time periods thereafter ${ }^{175}$

Figure 19: Schematic of improved GO synthesis

Figure 20: Water Contact Angle measurement for (a) Graphene oxide, (b) GO

dried under vacuum desiccator and (c) GO dried under mild heating. .54

Figure 21: After main oxidation step, two distinct layers were obtained floating graphite layer and the debris layer.

Figure 22: (a) Mixture after mechanical stirring of the large floating GO flakes, before centrifugation. After centrifugation, the solution showed 3 distinct portions (b) lower graphite portion with un-oxidised graphite, (c) middle portion with some un-oxidised graphite and (d) upper portion with GO flakes 56 Figure 23: SEM images of GO flakes on $\mathrm{SiO}_{2}$ from the (a) top (b) middle and (c) lower portion part of the GO synthesis mixture after centrifugation.

Figure 24: (a) SEM image of a large $\mathrm{GO}$ sheet on $\mathrm{SiO}_{2}$ substrate produced from the modified method. (b) Size distribution of GO flakes.

Figure 25: (Left) AFM image of GO and (Right) AFM height profile for the GO

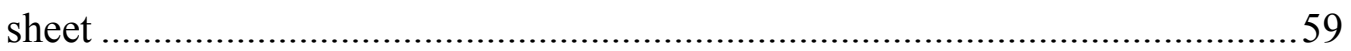

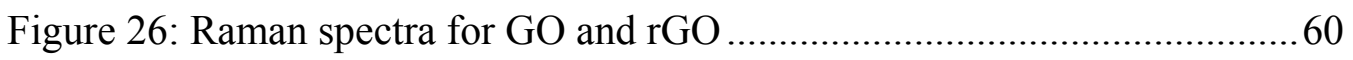

Figure 27: C1s XPS spectra for a) GO, b) reduced GO. Peaks corresponding to different carbon bonds were indicated

Figure 28: (a) Full and (b) magnified, image of Shadow mask design for FET fabrication

Figure 29: (a) Optical micrograph of the fabricated graphene device. (b) Output characteristics of the transistor device under different applied gate voltages. (c) Current - voltage transfer curve of bottom-gated graphene FET at a drainsource bias of $\mathrm{V}_{\mathrm{d}}=0.1 \mathrm{~V}$

Figure 30: Distribution of the hole mobility of graphene FETs .......................64

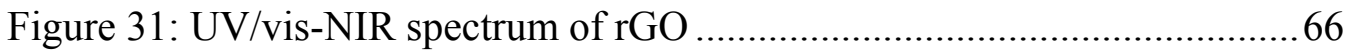

Figure 32: Distribution of the sheet resistance of graphene devices .................66 
Figure 33: Schematic of amperometric field effect transistor (FET) biosensor

setup

Figure 34: Electrical current output vs. sodium ion concentration input of the fully organic sodium sensor in response to (a) target sodium ions and (b) interference potassium ions.

Figure 35: Field emission scanning electron microscopy images of (a) graphene oxide flakes on silicon dioxide wafer (b) cotton thread after plasma treatment (c) graphene oxide on cotton thread taken under similar magnification .73 Figure 36: (a) Graphene oxide based sensor with integrated fabric pump. (b) A zoomed in image of the RGO transducer in the sensor. .74 Figure 37: Electrical resistance output vs. sodium ion concentration input of the fully organic sodium sensor. .75

Figure 38: SEM image of self-terminating carbon nanotubes (CNT) on functionalized $\mathrm{SiO}_{2}$ substrate. .81

Figure 39: Schematic illustration of the fabrication of the CNT-GO layers......83 83

Figure 40: Schematic of CNT-GO layers and its sandwich structure... .83

Figure 41: Time dependent study of GO coverage on CNT under constant reflux of a) 1, b), 3, c) 5 and d) 9 hours .84

Figure 42: SEM image of control experiment conducted with GO and CNT at room temperature after 9 hours.

Figure 43: Photographic image of a) GO solution b) $\mathrm{GO}$ with $\mathrm{NaCl}$ c) $\mathrm{GO}$ with

$\mathrm{NaOH}$ d) $\mathrm{NaOH}$ with 3 hour reflux

Figure 45: SEM images with $1 \mathrm{keV}$ showing CNT-GO hybrid layer at a) $800 \mathrm{x}, \mathrm{b}$ ) 16000x and 55000x magnification

Figure 46: SEM image of a) heavily wrinkling b) and its higher magnification on rGO surface after 5 hours reflux. The wrinkling accounted for $20 \%$ of surface area and was interconnected 88

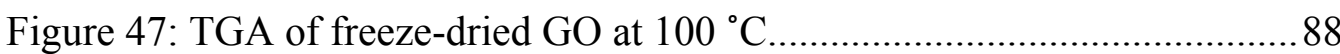

Figure 48: An atmospheric pressure CVD setup with alcohol vapors for growth of $\mathrm{rGO}$ 94

Figure 49: SEM image of a) before ethanol CVD treatment GO flakes [highlighted in purple] on $\mathrm{SiO}_{2}$ substrate with $c a$. $60 \%$ coverage b) after a 30minutes CVD treatment RGO flakes [highlighted purple] and the extended rGO growths [highlighted in yellow] with a total $c a .75 \%$ coverage. c) Highmagnification image on the new growths bridging between two rGO flakes....95 Figure 50: TEM image of pre-existing rGO (darker) and new grown rGO (highlighted in yellow) 96 Figure 51: SEM image of a) GO flakes before and b) rGO flakes and extended rGO growths [highlighted in yellow] after 2 hours ethanol CVD treatment. The size and features of the GO were preserved after the CVD treatment.

Figure 52: SEM images of (a) before ethanol CVD treatment GO flake templates on $\mathrm{SiO} 2$ substrate (b) after a 2 hour CVD treatment rGO flake with rGO new growths completely covering substrate (c) High-magnification image of the new grown flakes bridging a gap between rGO flakes. 
Figure 53: Electron microscope image of 2-hour treated (a) GO on lacey carbon (b) SAED patterns of reduced GO at the newly formed GO. .98 Figure 54: a) Optical micrograph [100x magnifications] of rGO flakes treated with 2-hours of ethanol CVD, showing contrast difference between rGO flake and out of flake areas. Raman spectra [488nm] on b) rGO flake and c) out of flake areas, with different CVD processing times, in comparison to GO and Highly Ordered Pyrolytic Graphite (HOPG)

Figure 55: Raman spectra of 2D band of HOPG with its 2D1 and 2D2 components deconvoluted.

Figure 56: (a) Optical microscope image of 2-hour processed reduced GO flakes on $\mathrm{SiO}_{2}$ substrate (b) Raman mapping at edges of rGO flakes showing $100 \%$ carbon coverage. (c) Raman spectrum of both on and out of rGO flakes.

Figure 57: XPS spectra and identified binding energies for a) GO and b) rGO after 15 mins and c) 120 mins of ethanol CVD treatment ...

Figure 58: C1s XPS spectra $(\mathrm{hv}=1486.6 \mathrm{eV}$ ) collected on (a) GO; and Reduced GO reduced in similar CVD setup with (b) 1 hour standard CVD treatment (c) 15 minutes CVD treatment with no ethanol (d) 1 hour CVD treatment with more $\mathrm{H}_{2}$ gas (e) 12-hour hydrazine reduced sample. The different components related to various chemical shifts of carbon bonds were indicated. 104 Figure 59: First derivative of the X-ray excited C KLL spectra of HOPG, CVD treated samples and diamond. The $\mathrm{D}$ value which corresponded to the area ratio of $\mathrm{sp}^{2}$ increases with increasing ethanol CVD process time.

Figure 60: AFM images with Root Mean Squared (RMS) roughness value and corresponding height profile of GO with different stages of CVD process. ... 108 Figure 61: Output characteristics $I_{d}-V_{d}$ (drain current- drain voltage) of the transistor device under different applied gate voltages and Id-Vg (drain currentgate voltage) transfer curve of bottom-gated graphene FET under sweeping $V_{g}$

Figure 62: Scanning electron microscope image of (a) before ethanol CVD treatment GO flakes with ca. $60 \%$ coverage and (b) after 30-minutes ethanol CVD treatment rGO flakes with ca. $80 \%$ coverage [GO flakes highlighted in purple and new growth highlighted in yellow, please refer to online version for colour] (c) High-magnification image on the new growths. Figure 63: (a) Output graph $\mathrm{I}_{\mathrm{d}}-\mathrm{V}_{\mathrm{d}}$ (drain current- drain voltage) under different applied gate voltages and (b) $\mathrm{I}_{\mathrm{d}}-\mathrm{V}_{\mathrm{g}}$ (drain current-gate voltage) transfer curve of the CVD-processed rGO device under vacuum and room temperature. [Please refer to softcopy version for colour]

Figure 64: Ultra-violet Raman spectra of diamond, GO, different ethanol CVD processed rGO and HOPG. The intensities of diamond and HOPG were scaled down to allow fitting into graph..... 116 Figure 65: Schematic showing the growth sequence of rGO from a GO template during ethanol CVD treatment 119 
Figure 66: SEM images of (a) GO flakes before ethanol CVD treatment with ca. $60 \%$ coverage (b) reduced GO flakes after 30-minutes CVD treatment with ca. $75 \%$ coverage in total. (c) high-magnification on the new growths between two reduced GO flakes. [GO flakes and new carbon growths were highlighted in purple and yellow respectively, please refer to online version for colour]..... 124 Figure 67: Schematic showing (a) top-view and (b) side-view of fabricated FET devices with silicon well suitable for analyte testing.

Figure 68: Detection characteristics of rGO FET biosensors. (a) Source-drain current $\left[\mathrm{I}_{\mathrm{sd}}\right]$ vs. gate voltage $\left[\mathrm{V}_{\mathrm{G}}\right]$ plot with different concentration of IL-6 protein, showing decreasing $\mathrm{I}_{\mathrm{SD}}$ at increasing IL-6 addition; (b) ISD vs. time with addition of $0-300 \mathrm{pg} / \mathrm{ml}$ of IL-6; and (c) real-time $\mathrm{I}_{\mathrm{SD}}$ plot, showing negligible effect when BSA interference protein was added.

Figure 69: The detection of spiked rhIL-6 in real and artificial sweat in study 2 .

a) The standard curve for spiked recovery of IL- 6 in artificially made sweat (filled square) and real sweat (filled triangle) indicated that there was no interference of the sweat matrix on the detection of IL- 6 across a physiological range. b) Plasma IL-6 concentration did not differ between resting and exercise trials or in response to exercise. 


\section{LIST OF TABLES}

Table 1 Electrochemical means of producing GO, its associated conditions, yield and characterizations were summarized. The table was arranged in decreasing percentage yield order and concentration of GO obtained. Table adapted from Ref. ${ }^{71}$

Table 2 Comparison of size and hole mobility of GO synthesized from chemical approach from graphite (arranged according to average and maximum size of obtained flake).

Table 3 The values of $\mathrm{C} 1 \mathrm{~s} / \mathrm{O} 1 \mathrm{~s}$ (atomic ratios) of thermal-reduced and hydrazine-reduced GO samples obtained from XPS survey spectra ${ }^{106}$

Table 4 Advantages and disadvantages of Whole Body Washdown vs. Regional

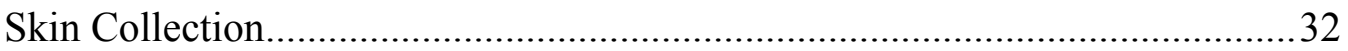

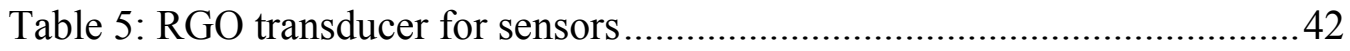

Table 6: A comparison of currently available and practical sodium sensors ....43

Table 7: Comparison of GO yield concentration between oven-dried and vacuum-dried pre-oxidised samples (the GO solution was obtained after centrifuging at $5000 \mathrm{G}$ for $3 \mathrm{mins}$ ).

Table 8: Exfoliated GO concentration obtained after the samples were ovendried, vacuum-dried and membrane-dialysed

Table 9: Comparison of ultrathin electrodes prepared from various reduced GO

sheets.

Table 10: Comparison of existing CNT-rGO hybrid film methods.

Table 11: Comparison of existing CNT-rGO film transparency and sheet resistance

Table 12: Atomic percentage of different carbon bonds identified by XPS on mono-layered $\mathrm{GO}$ and $\mathrm{rGO}$ reduced under different conditions

Table 13: D parameter and $\mathrm{sp}^{2}$ percentage area of different carbon samples .. 107 Table 14: Electrical measurements of rGO processed under different conditions

Table 15: D/G Raman spectrum peak intensity, AFM height profile and 4-point probe sheet resistance for GO and $\mathrm{rGO}$ reduced under different conditions ... 117 Table 16: Comparison of reported biosensors to IL-6 128 


\section{CHAPTER 1 LiTERATURE REVIEW}

This chapter summarized the evolution of the structure, properties and synthesis methods of Graphene Oxide (GO) with an emphasis on sensing application. Then, the theoretical and experimental investigation of recent GO synthesis methods were reviewed and discussed.

Following, the chapter summarized the production of Interleukin-6 (IL-6) and sodium ions in circulation and sweat respectively. Next, their relevance to both sports performance indication and potential health risks were discussed. Finally, the chapter end with summary of GO's potential in biosensors.

\subsection{OVERVIEW OF GRAPHENE AND GRAPHENE OXIDE}

Graphene was a relatively new 2-dimensional material that had attracted significant attention due to its unique physiochemical properties and potential applications. The method for the preparation of this single layer carbon was discovered in $2004^{1}$. Since then, graphene's extraordinary carrier mobility, chemical, thermal and optical properties had been highlighted ${ }^{2-4}$ and researched heavily (Figure 1). However the semi-metal zero band-gap electronic structure of both chemical vapor deposition (CVD) and mechanically-exfoliated graphene limited its use in electronic, sensing and optical applications ${ }^{5}$. Some approaches to create a band-gap includes post-processing of graphene such as lateral confinement ${ }^{6}$, strain engineering ${ }^{7}$, breaking inversion symmetry in bilayered graphene ${ }^{8}$ and the usage of $\mathrm{GO}^{9}$. 


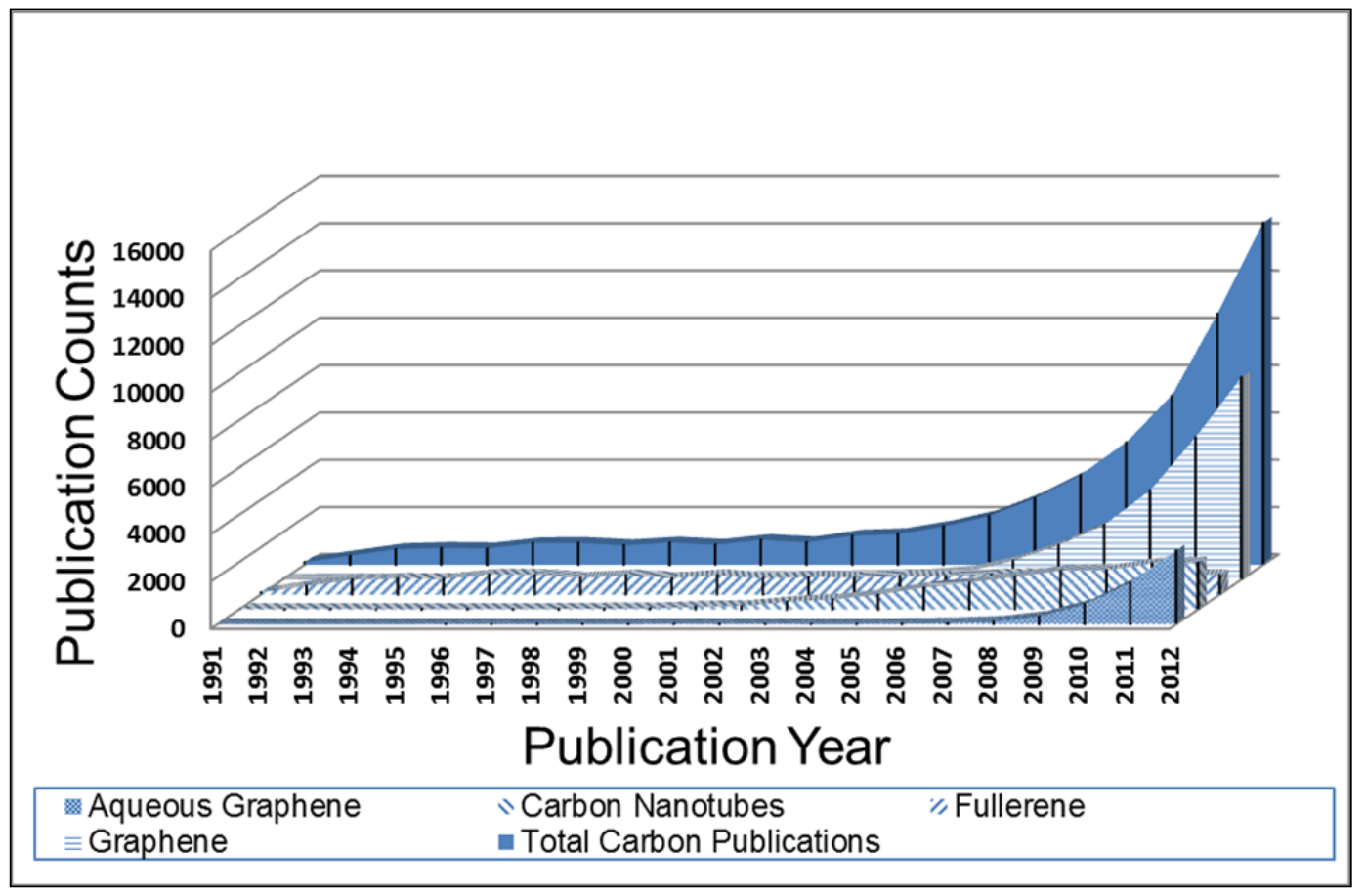

Figure 1: Number of publications on carbon-based materials over past 20 years. [Source: Web of Science, ISI]

In contrast to the other methods, GO stood out particularly due to its solutionbased, scalable, facile and low-cost synthesis. Thus there was an increasing interest in this material in recent years ${ }^{10-13}$. The subsequent sections of this chapter aimed to introduce the reader to the properties of GO and state of the art methods to synthesize them. After GO was obtained, a reduction step was needed to reinstate the structure to reduced GO before it could be used for electronic and bio-sensor applications. Thus a section on the properties of reduced GO and its methods to synthesize them was discussed. Finally at the end of the chapter, the application of reduced GO in biosensor devices was demonstrated.

Before reading further, it is vital to know that one of the earliest known works on GO was documented by Brodie ${ }^{14}$ in 1859. In his work, potassium chlorate $\left(\mathrm{KClO}_{3}\right)$ and nitric acid $\left(\mathrm{HNO}_{3}\right)$ were added to graphite. He found that the product (GO) was dispersible in water, not in acid and termed the product "graphic acid". 
In addition to Brodie's term "graphic acid" for GO, there were a lot of other terms which were used interchangeably in different books, journals and communications to refer to GO, i.e. graphitic acid ${ }^{15}$, graphitic oxide ${ }^{15}$, aqueous graphene $^{16}$, graphon ${ }^{14}$, chemically exfoliated graphene ${ }^{17}$, chemically modified graphene $^{18}$ and graphene oxide ${ }^{9}$. In this thesis, single-layer Graphene Oxide was referred to as $\mathrm{GO}$.

\subsection{STRUCTURE AND PROPERTIES OF GRAPHENE OXIDE}

The chemical structure of GO was still being debated upon. This was because GO was a soft matter and had a non-stoichiometric molecular formula. Soft materials had a large internal degree of freedom caused by a competition between the entropic and the enthalpic contributions to the free energy that occurred even at room-temperature ${ }^{19}$. GO films could undergo spontaneous modifications and reduction with a relaxation time of $\sim 35$ days. After this selflimiting process, the film was deprived of epoxy groups but remained rich in hydroxyl groups ${ }^{20}$. This relaxation corresponded to a drop of $\mathrm{C} / \mathrm{O}$ ratio from 0.44 to $0.38^{20}$. After the self-limiting relaxation, the GO's atomic structure was still metastable. GO's structure would change again with exposure to thermal heat $^{21}$ or ultra-violet light ${ }^{22}$ into a more stable form; thus understanding only their atomic bonding becomes inadequate ${ }^{23,24}$. How the GO behaved in different environment must be understood as well. A general structure but not a definitive one could be used to describe GO. The next few pages presented some well-accepted general structures of GO.

Historically, many models of GO had been proposed since Brodie's original graphic acid synthesis ${ }^{14}$. Brodie stated that GO had a molecular weight of 33, suggesting that it was regular and crystalline ${ }^{14}$. Consequently, many others had supported this notion that the structure of GO was both regular and crystalline $e^{25,}$ ${ }^{26}$. Hofmann ${ }^{27}$ then proposed that only epoxy $(\mathrm{C}-\mathrm{O}-\mathrm{C})$ groups were present on GO. Ruess ${ }^{28}$ suggested the presence of hydroxyl (-OH) thereby accounting for the hydrogen content. Scholz and Boehm ${ }^{29}$ later added that ketone $(\mathrm{C}=\mathrm{O})$ 
groups were present. Then in 1998, Lerf et al. ${ }^{30}$ was one of the first to suggest that GO could not have a regular structure but agreed with earlier suggestions that different hydrogen and oxygen groups were present on GO.

Lerf et al. ${ }^{30}$ used NMR spectroscopy data to suggest a model for GO which was

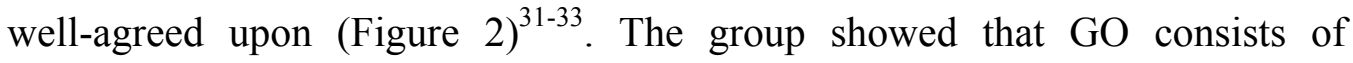
competing areas of un-oxidized benzene rings and aliphatic six-membered rings; with random epoxy (C-O-C) and hydroxyl $(\mathrm{C}-\mathrm{OH})$ groups on the basal planes; and carboxyl $(\mathrm{C}-\mathrm{OOH})$ groups on the edges. The report also assigned the NMR lines around 60, 70 and $130 \mathrm{ppm}$ to those of epoxy (1,2-ether), hydroxyl and $\mathrm{sp}^{2}$ carbon respectively. These values had since been widely accepted ${ }^{34,35}$. Due to the presence and distribution of these oxygen moieties, GO monolayers could be dispersed and suspended in aqueous solutions.

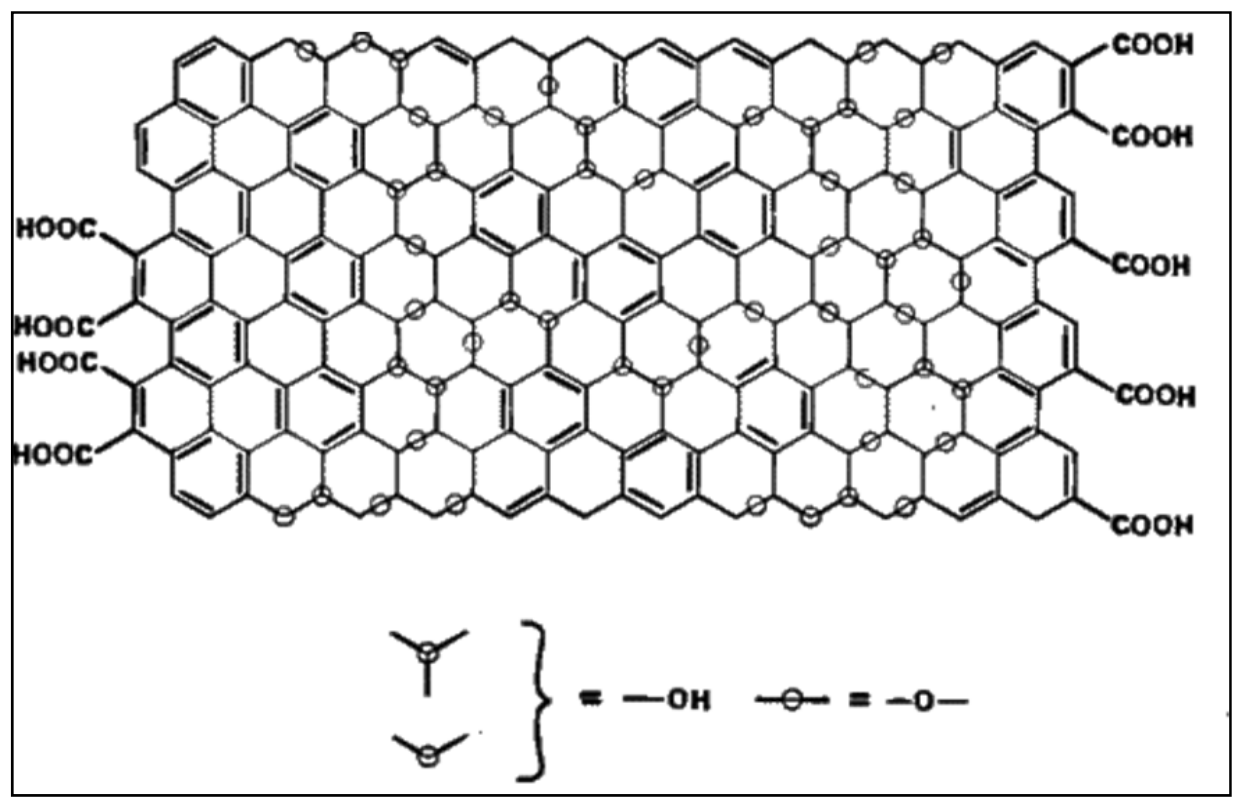

Figure 2: Structural model of GO proposed by Lerf et al. ${ }^{30}$

Then in 2006, Szabo et al. ${ }^{36}$ proposed that since high area of $\mathrm{C}=\mathrm{O}$ satellite was present in the XPS spectra and the carboxylate peaks were lacking in the IR spectrum of sodium salts of $\mathrm{GO}$, therefore the $\mathrm{C}=\mathrm{O}$ groups must also exist as ketones and/or quinones on the basal planes, in contrast to Lerf's model where 
$\mathrm{C}=\mathrm{O}$ groups only exist on the edges. The group also suggested that $\mathrm{GO}$ sheet contained two domains (a trans-linked cyclohexane chairs and a corrugated hexagon ribbons) and that the slight tilting angle between the boundaries of these two regions could cause wrinkling of GO as shown in Figure 3.

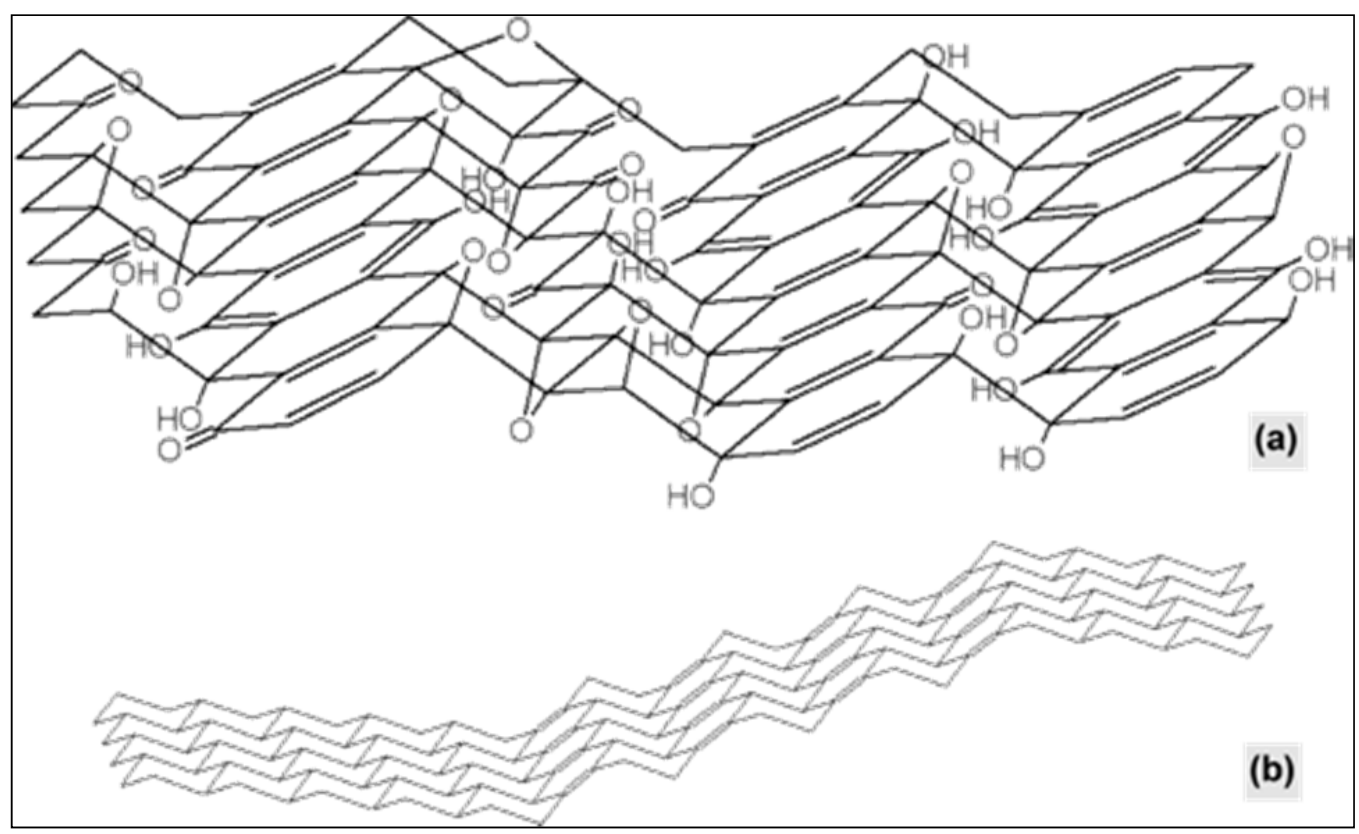

Figure 3: (a) Surface species of GO proposed by Szabo with the inclusion of $(C=O)$ in basal planes (b) Boundaries between domains of GO causes folded carbon skeletons ${ }^{36}$

Subsequently, Gao et al. ${ }^{37}$ using MAS ${ }^{13} \mathrm{C}$ NMR reported that, in addition, five and six-membered lactol rings were present on the edges while esters of tertiary alcohols were present on the basal planes. The relative ratios were likely to be 115 (hydroxyl and epoxy): 63 ( $\mathrm{sp}^{2}$ carbon): 9 (ketone carbonyl): 10 (lactol, ester and acid carbonyl): 3 (lactol $\mathrm{O}-\mathrm{C}-\mathrm{O}$ ) on GO. However, the main functional groups in GO were still that of epoxy, hydroxyl and carbonyl ${ }^{34,37}$.

The arrangement of the oxygen moieties on the GO were random and made the sheet predominately amorphous ${ }^{38}$. The thickness of a fully exfoliated individual GO sheet was $\sim 1 \mathrm{~nm}^{39-41}$. This was $\sim 3$ times larger than the $0.334 \mathrm{~nm}$ interlayer distance of pristine graphene due to the presence of adsorbed water from the atmosphere and various oxygen functional groups on its basal planes. The 
oxidation of $\mathrm{sp}^{2}$ to $\mathrm{sp}^{3}$ bonds also created stress and this in turn created wrinkles in the structure of an otherwise flat structure ${ }^{36}$; carbon atoms that had the hydroxyl (-OH) group attached had a slightly distorted tetrahedral configuration $^{30}$. Thus the average roughness of the GO sheet was measured to be about $6 \AA^{38}$. Since all known C-O bond lengths were less than $3 \AA$, the other $3 \AA$ or more variation in the roughness value must be caused by the lattice distortions in the $\mathrm{GO}^{42}$.

GO, unlike meso-graphite, was electrically insulating. TEM images (Figure 4) showed that GO consisted of ordered $\mathrm{sp}^{2}$ conductive clusters isolated within $\mathrm{sp}^{3}$ insulative matrix with variable $\mathrm{C} / \mathrm{O}$ moieties. This isolation and the absence of percolation pathways among the $\mathrm{sp}^{2}$ carbon clusters prevented traditional carriers to flow through, thus making GO an insulator ${ }^{43}$. While sufficient oxidation was required to exfoliate the GO sheets from graphite, it also increased the percentage of the $\mathrm{sp}^{3}$ to $\mathrm{sp}^{2}$ ratio ${ }^{44}$. So instead of electron flowing through the $\pi$ bond, the carrier in GO now hop between regions of conductive $\mathrm{sp}^{2}$ areas and this temperature-dependent conductance could be described in the following equation according to ref. ${ }^{45}$.

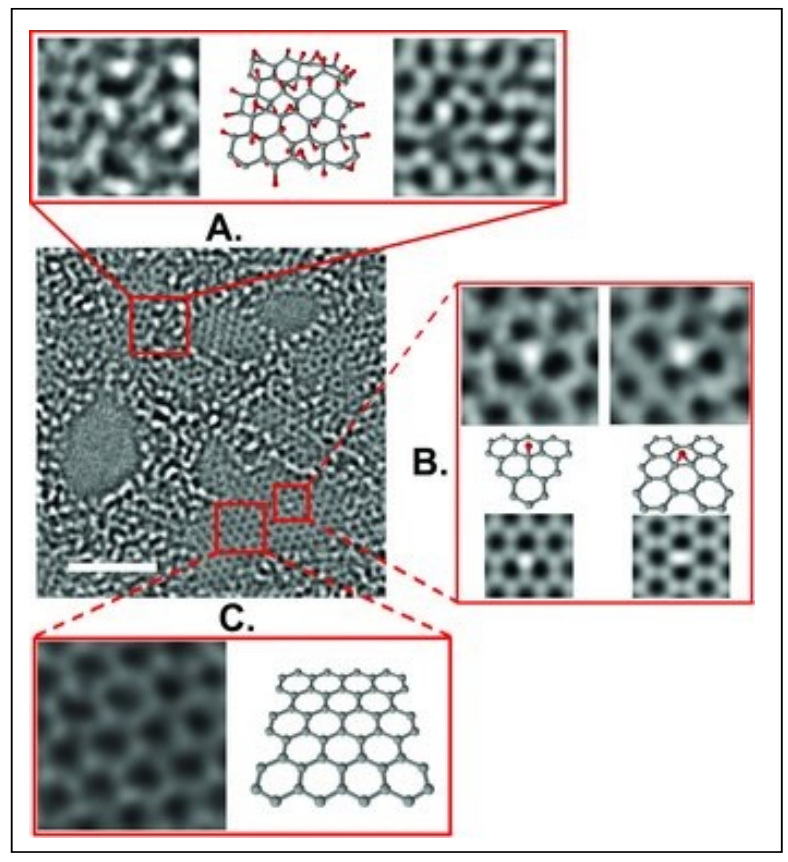

Figure 4: Aberration-corrected TEM image of GO, with scale bar 2nm, showing that sp ${ }^{2}$ islands were isolated in a $\mathbf{s p}^{3}$ matrix. Magnified images of (a) oxidized sp3 (b) possible mobile oxygen functionalities (c) graphitic $\mathrm{sp}^{2}$ of $\mathrm{GO}^{46}$ 
Where $\mathrm{B}$ was the hopping parameter, $\mathrm{N}\left(\mathrm{E}_{\mathrm{F}}\right)$ was the density of states near the Fermi level, $\mathrm{L}_{1}$ was the localization length of the electronic wave functions, $\mathrm{K}$ was the Boltzmann's constant. Equation (1) represents the usual 2D variablerange hopping and the Equation (2) represents a purely field-driven conduction independent of temperature.

\section{Equation 1: 2D variable-range hopping}

$\mathrm{G}(\mathrm{T})=G_{1} \exp \left(-\frac{B}{T^{1 / 3}}\right)+G_{0}$

\section{Equation 2: Hopping parameter equation}

$B=\left[\frac{3}{k N\left(E_{F}\right) L_{1}^{2}}\right]^{1 / 3}$

Having understood the structure and properties of GO, we can then appreciate the evolution and the state of the art synthesis methods to obtain GO in the following section. 


\subsection{METHODS IN GRAPHENE OXIDE SYNTHESIS}

New synthesis methods and characterization was of increasing importance for molecular electronics, sensing and battery applications in which GO of better quality could improve the reported performance results with all else held constant. Not all GO were created equal. In this section, the evolution of GO synthesis methods to produce larger flakes with higher electrical conductivity and carrier mobility were explored and discussed.

Generally, GO can either be synthesized using the bottom-up or the top-down method $^{47-51}$. The bottom up method involved oxidation and dehydration of similar scaled nanometer thick graphene $\mathrm{e}^{52}$ and sugars ${ }^{47}$; whereas the top-down method involved splitting of millimeter thick graphite ${ }^{53}$ (Figure 5).

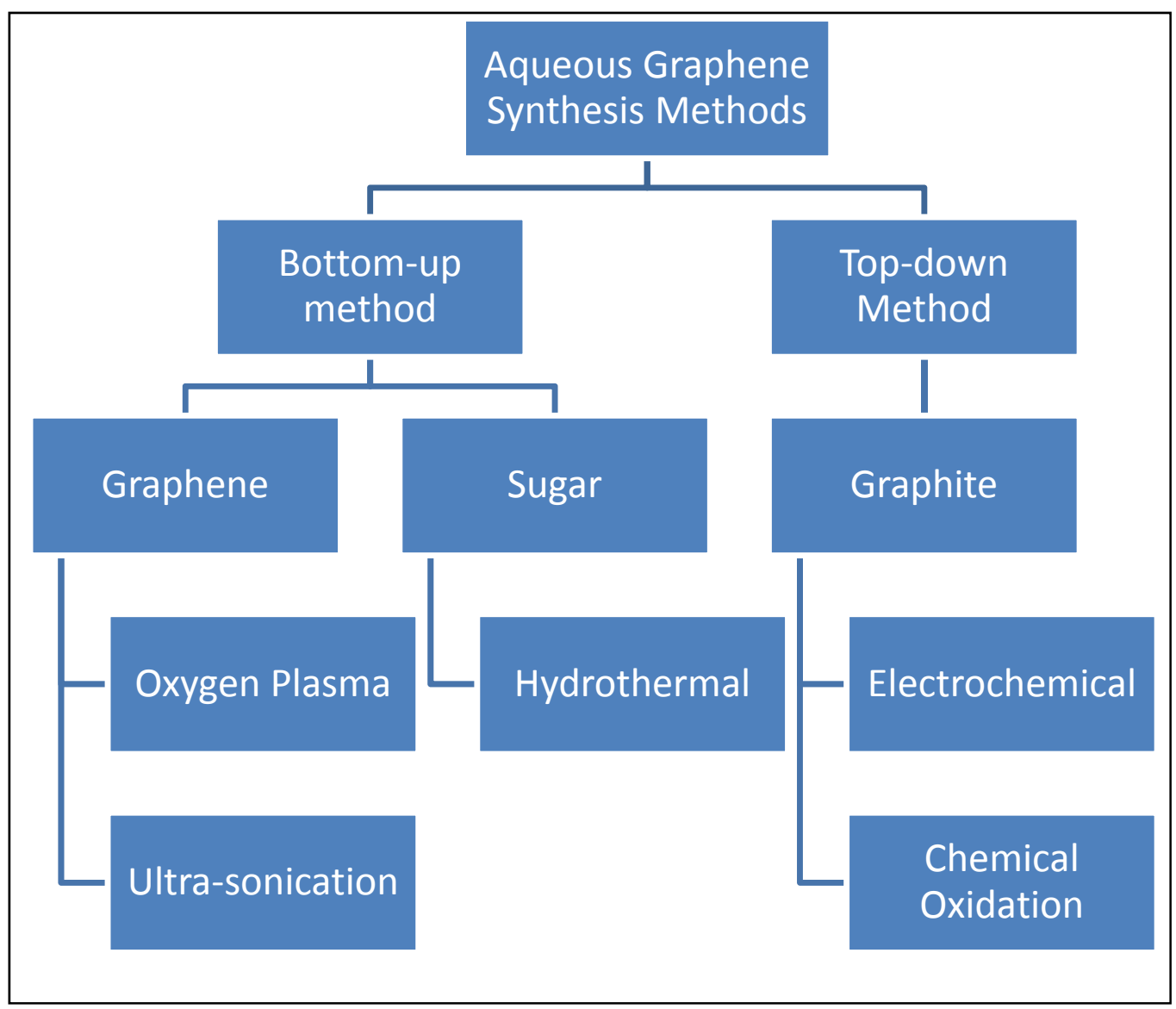

Figure 5: Schematic of GO synthesis methods 


\subsection{1 ВотTOM-UP}

\section{Oxygen Plasma and Ultra-sonication}

In the bottom-up method, pristine graphene could be post-treated to obtain GO. Briefly, the pristine graphene could be obtained using mechanical exfoliation ${ }^{4}$, 54, 55, sublimation ${ }^{56,57}$ or $\mathrm{CVD}^{58,59}$. Mechanical exfoliation from highly oriented pyrolytic graphite (HOPG) produced graphene with the best structural integrity. It was also an easier method to produce graphene; however it had a very low throughput and produced small-sized graphene sheets unsuitable for practical usage. Sublimation of silicon out of $\mathrm{SiC}$ wafer at high temperature produced high-quality large area graphene but the costs of $\mathrm{SiC}$ wafers were high ${ }^{56}$. In sublimation process, silicon sublimates from the SiC surface leaving carbon behind to grow into larger domains. The thickness and quality of the carbon growth was dependent on both the silicon sublimation rate and pressure of decomposition $^{56,60}$.

CVD method of producing graphene was the more common, scalable and reproducible approach to graphene, but it was complicated and time-consuming. The CVD growth of graphene required a catalyst as substrate such as $\mathrm{Ni}^{61}$ or $\mathrm{Cu}^{62}$ on which graphene will grow during the CVD process. High carbon solubility substrate such as $\mathrm{Ni}$ thin film allowed the carbon to diffuse into the metal thin film at growth temperature and upon cooling, precipitate out of the substrate. Whereas low carbon solubility substrate such as $\mathrm{Cu}$ thin film catalytically decomposed methane into radicals and then allowed the carbon to grow into graphene by an adsorption process on the surface ${ }^{63}$.

After obtaining the pristine graphene, oxygen plasma $\operatorname{etch}^{52}$ and ultrasonication ${ }^{64}$ could be applied to obtain GO via the bottom-up method. These post-treatment of graphene, necessary for various end-applications, gave a band-gap in the otherwise semi-metallic material. The post-treatment steps added oxygen moieties by breaking the $\mathrm{C}-\mathrm{C}$ bonds thus changing the surface characteristic from hydrophobic to hydrophilic. The amount of C-C bond breakages on the graphene was proportional to the treatment time $\mathrm{e}^{52,64}$ and thus the treatment time could be used to control the amount of oxygen within the 
obtained GO. Moreover, oxygen plasma etch on graphene could be controlled layer-by-layer ${ }^{65}$ and increased process time could be used to pattern graphene ${ }^{66}$. Using ultra-sonication however, added oxygen moieties predominantly at the edges rather than the basal planes ${ }^{64}$. Therefore post-processing of graphene was the method of choice for controlling the amount and location of oxygen moieties of the obtained GO.

\section{Hydrothermal method}

Alternatively, GO could also be synthesized bottom-up via hydrothermal method starting from smaller molecules. Tang et al. ${ }^{47}$ were able to obtain GOlike materials from sugars such as fructose and glucose. In this study, the sugar molecules were dehydrated under hydrothermal conditions and yielded GO-like sheets of $20 \mu \mathrm{m}$ average size. However, the reported electron mobility of $1.07 \times 10^{-5} \mathrm{~cm}^{2} / \mathrm{Vs}$ was still much lower than GO synthesized using top-down chemical methods ${ }^{9}$. The main advantage of using this sugar dehydration synthesis method was because of its environmental friendliness.

\subsubsection{TOP-DOWN}

Along the first and second axis in graphite, the carbon atoms were held to its neighbouring carbon atoms by strong covalent bonds. However, along the third axis, the covalent-bonded sheets were held together by weak van der Waals bonding $(\sim 40-70 \mathrm{meV})^{67}$. Top-down synthesis methods aimed to increase the interlayer distance either by oxygen moieties addition or electric potential usage to overcome this weak bond and thus separate single sheets from the bulk graphite yielding GO. Top-down synthesis methods to obtain GO were more commonly used compared to the bottom-up methods.

It was to be noted that raid thermal evaporation and expansion intercalation of graphite did produce graphite-like nanoplatelets. However, it did not result in the complete exfoliation of graphite into individual GO sheets ${ }^{40,68}$. One of the 
common intercalation compound used in graphite was sulphuric-acid. These exfoliated nanoplatelets did possess oxygen functional groups on the surface, like GO, but they were multi-layers ${ }^{69}$. The thickness distribution of the graphite flakes ranged within a batch from to $30-100 \mathrm{~nm}^{40,70}$. In contrast, a GO sheet had an average thickness of $\sim 1 \mathrm{~nm}^{39}$.

\section{Electrochemical}

The electrochemical synthesis method worked by oxidising the graphite working electode. A constant potential or a cyclic voltametry was applied to the working electrode and this led to the formation of GO via the intercalation of anions from the electrolyte ${ }^{71}$ (Figure 6). Anodic potential in either aqueous (e.g. acid in water $^{53}$ ) or non-aqueous (e.g. mixture of ionic liquids ${ }^{72}$ ) electrolytes could be used in the electrochemical method of preparing GO. Carbon dioxide and carbon monoxide gas could be produced in side-reactions if the potential was set too high ${ }^{73}$. The properties of electrochemical-synthesized GO could be tuned by adjusting the electrolysis specifications and electrolytes ${ }^{74}$. Usage of a three-electrode cell system could allow greater control of potential of the working electrode and also precise control on the thickness of exfoliated $\mathrm{GO}^{71}$, 74

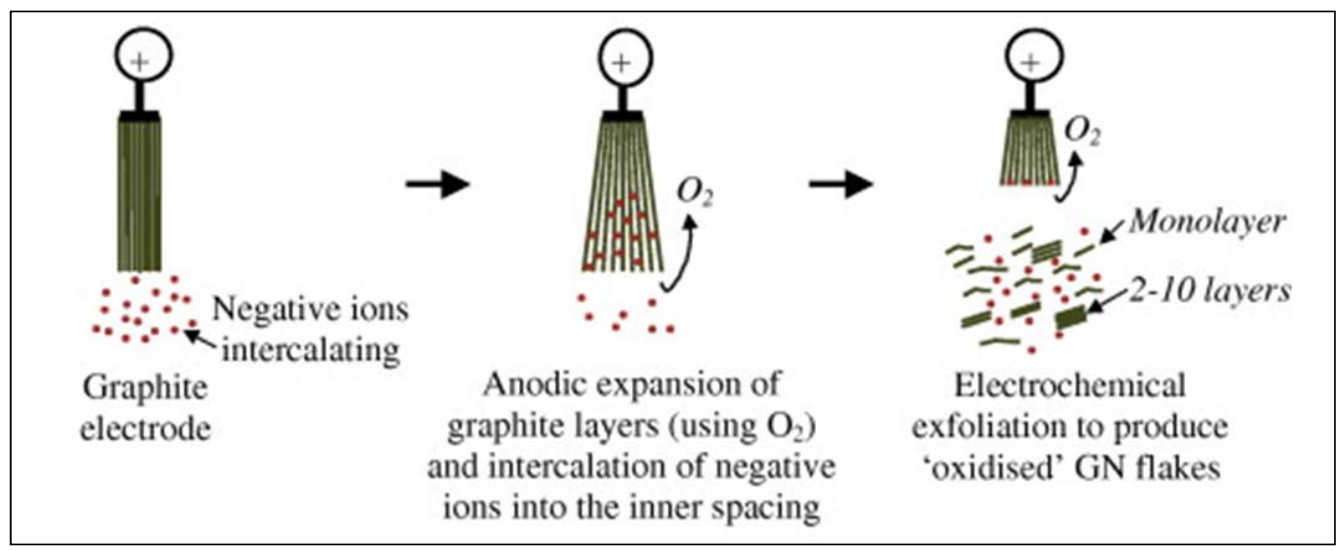

Figure 6: Schematic showing the anodic oxidation, intercalation and exfoliation steps to produce single and multilayer $\mathbf{G O}^{71}$ 
Parvez et al. $^{53}$ reported that electrochemically exfoliating of graphite could yield $80 \%$ of $1-3$ layers GO flakes with lateral size $5-10 \mu \mathrm{m}$ and $\mathrm{C} / \mathrm{O}$ ratio of 12.3; the working electrodes, counter-electrodes and electrolytes used were natural graphite flakes, platinum wire and $0.1 \mathrm{M}$ sulphuric acid solution respectively. This was one of the highest GO yield obtained using electrochemical method in aqueous solution. Some of the electrochemical means of producing GO sheets and its associated yield were summarized in Table 1. 
Table 1 Electrochemical means of producing GO, its associated conditions, yield and characterizations were summarized. The table was arranged in decreasing percentage yield order and concentration of GO obtained. Table adapted from Ref. ${ }^{71}$

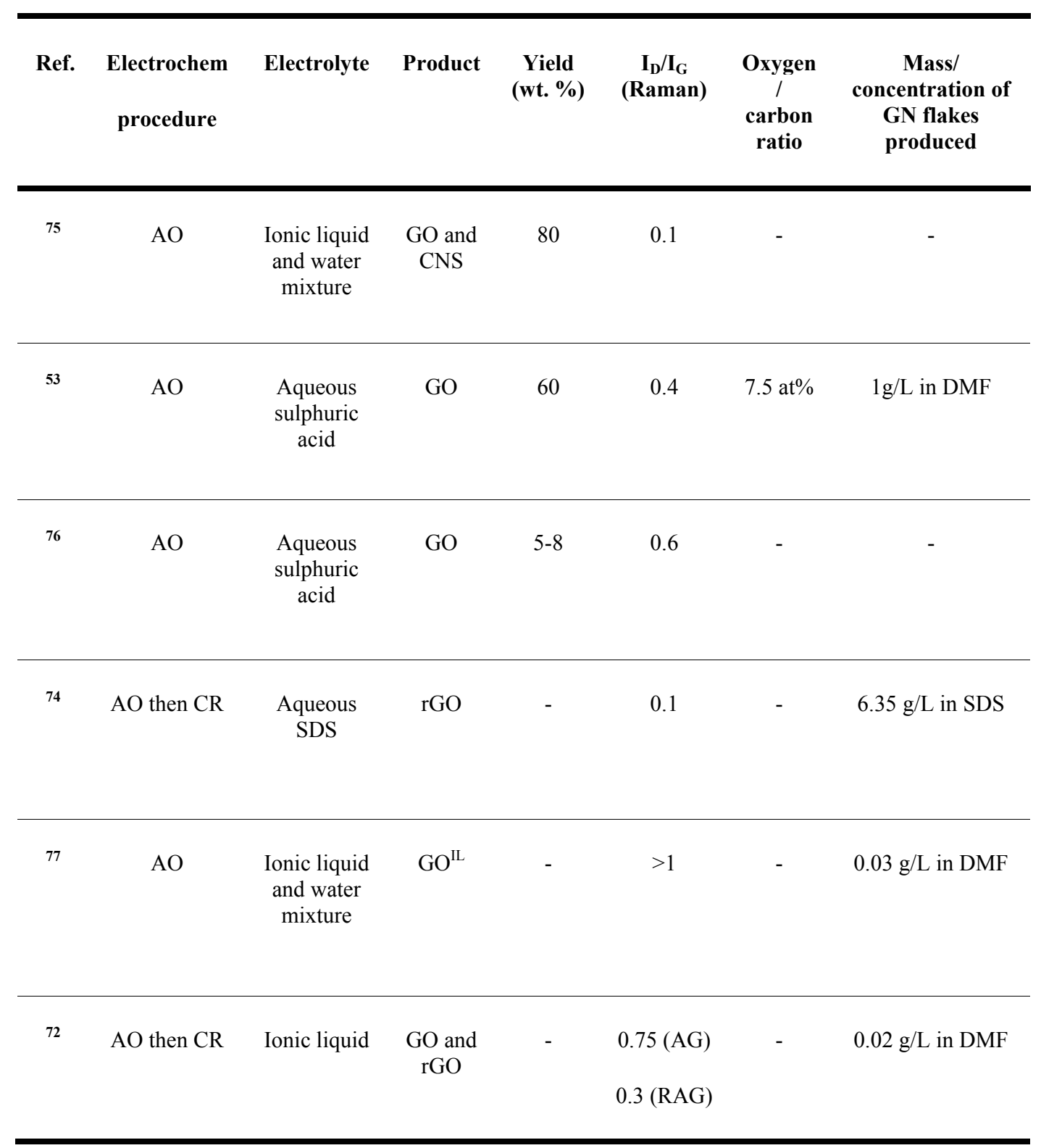

AO: Anodic Oxidation, CR: Cathodic Reduction, GO: Graphene Oxide, RAG: Reduced GO, $\mathrm{GO}^{\mathrm{IL}}$ : Ionic-liquid functionalized GO, CNS: carbon nano-structures, DMF: N,Ndimethylformamide, SDS: sodium dodecyl sulphate. 


\section{Chemical}

The earliest top-down method to obtain the GO was through chemical oxidation from natural graphite. The three most well-known standard chemical methods were the Staudenmaier's ${ }^{78}$, Hofmann's ${ }^{79}$ and Hummer's ${ }^{15}$ method. All 3 methods required graphite to be oxidized in strong acid and oxidants. Chemical synthesis methods were highly suitable for facile, low cost and large scale production of graphene. It was also compatibility with existing semiconductorbased electronics and thus was the most promising route to produce amperometric biosensors.

The earliest known work on GO was documented by Brodie ${ }^{14}$ in 1859 . In this work, potassium chlorate $\left(\mathrm{KClO}_{3}\right)$ and fuming nitric acid $\left(\mathrm{HNO}_{3}\right)$ were added to graphite to obtain GO. Fuming nitric acid was used as it was a strong oxidizing agent that can react with the aromatic carbon ring. Then in 1898, Staudenmaier ${ }^{78}$ added concentrated sulphuric acid to increase the acidity of the mixture and performed the experiment in a single reaction vessel ${ }^{31}$.

Subsequently, Hofmann's method $^{79}$, reported in 1937, further improved Staudenmaier's by using a less hazardous concentrated nitric acid instead of fuming nitric acid. This process still took a week and the use of nitric acid produces nitrous gases which were toxic and makes the synthesis route hazardous.

Later, Hummer's method ${ }^{15}$, reported in 1958, made the chemical synthesis process more efficient and less hazardous because the production of nitrous gases was avoided and the entire oxidation-process required less than two hours. The method involved treating graphite with concentrated sulphuric acid, sodium nitrate and potassium permanganate only. Hummer's method or its modified form was, at point of report, the most common method to get GO.

Although Hummer's method was less hazardous, nevertheless, the reaction still had to be performed with care; as the reaction between sulphuric acid and potassium permanganate formed reactive dimanganese-heptoxide, which was known to detonate when heated to above $55^{\circ} \mathrm{C}^{80}$. Equations 3 and 4 showed the 
reaction between sulphuric acid and potassium permanganate to form dimanganese heptoxide (adapted from references ${ }^{31}$ and ${ }^{81}$ ).

Equation 3: Reaction of potassium permanganate to form $\mathrm{MnO}_{3}{ }^{+}$cation

$\mathrm{KMnO}_{4}+3 \mathrm{H}_{2} \mathrm{SO}_{4} \rightarrow \mathrm{K}^{+}+\mathrm{MnO}_{3}^{+}+\mathrm{H}_{3} \mathrm{O}^{+}+3 \mathrm{HSO}_{4}^{-}$

Equation 4: Reaction of $\mathrm{MnO}_{3}{ }^{+}$cation to form dimanganese-heptoxide

$\mathrm{MnO}_{3}^{+}+\mathrm{MnO}_{4}^{-} \rightarrow \mathrm{Mn}_{2} \mathrm{O}_{7}$

Different chemical synthesis methods yielded different GO oxidation levels and properties. Graphene synthesized from Staudenmaier's, Hofmann's and Hummer's method with the same subsequent reduction technique, resulted in products that yielded moderately similar properties except Hummer's; where the (1) GO product had highest degree of oxidation (2) reduced GO exhibited higher heterogeneous electron transfer rate (3) structure showed presence of nitrogen moieties (4) electrochemical response showed greater difference ${ }^{82}$. Although Brodie's method yielded lower contamination and higher quality graphite, the final $\mathrm{pH}$ of the product needed to be increased by addition of $\mathrm{NaOH}$ or $\mathrm{KOH}$ to improve exfoliation ${ }^{83}$. The reagents used in the oxidation method had influences on the properties of the resulting GO.

Beside the reagents used in the different oxidation method, the resulting GO was also heavily dependent on the size of the precursor graphite crystal, oxidation time and graphite crystal quality. The maximum size of GO obtained was limited to the largest size of graphene domains in the graphite flake from which it was chemically synthesized ${ }^{43}$.

Oxidation time had a direct influence on the resulting GO. As oxidation proceeded, the oxygen moieties on graphite increased and the graphite eventually lose the metallic lustre and became a brown yellowish sludge. Also, with longer oxidation time, the hydroxyl groups in the basal planes converted to epoxide $^{84}$. 
The quality and structure of the graphite crystal varied widely because they were obtained from different manufacturers, batches and locations. Thus, it was not possible to compare the effects of oxidation on graphite across existing journal publications accurately. More comparative studies, such as one conducted by Poh et al. ${ }^{82}$ comparing a few well-established chemical synthesis methods, using same precursor graphite, were needed to shed light on the real effect of the specific oxidation step. A description on the characterization of the initial graphite precursor in reports could be very helpful for precursor standardization.

\section{Modified Hummer's Method}

Since the introduction of Hummer's method, many had improved on it. Thus the Hummer's method currently existed in many modified forms and the number of modified forms was set to increase in near future (Figure 7). This section highlighted a few well-cited modified Hummer's synthesis methods.

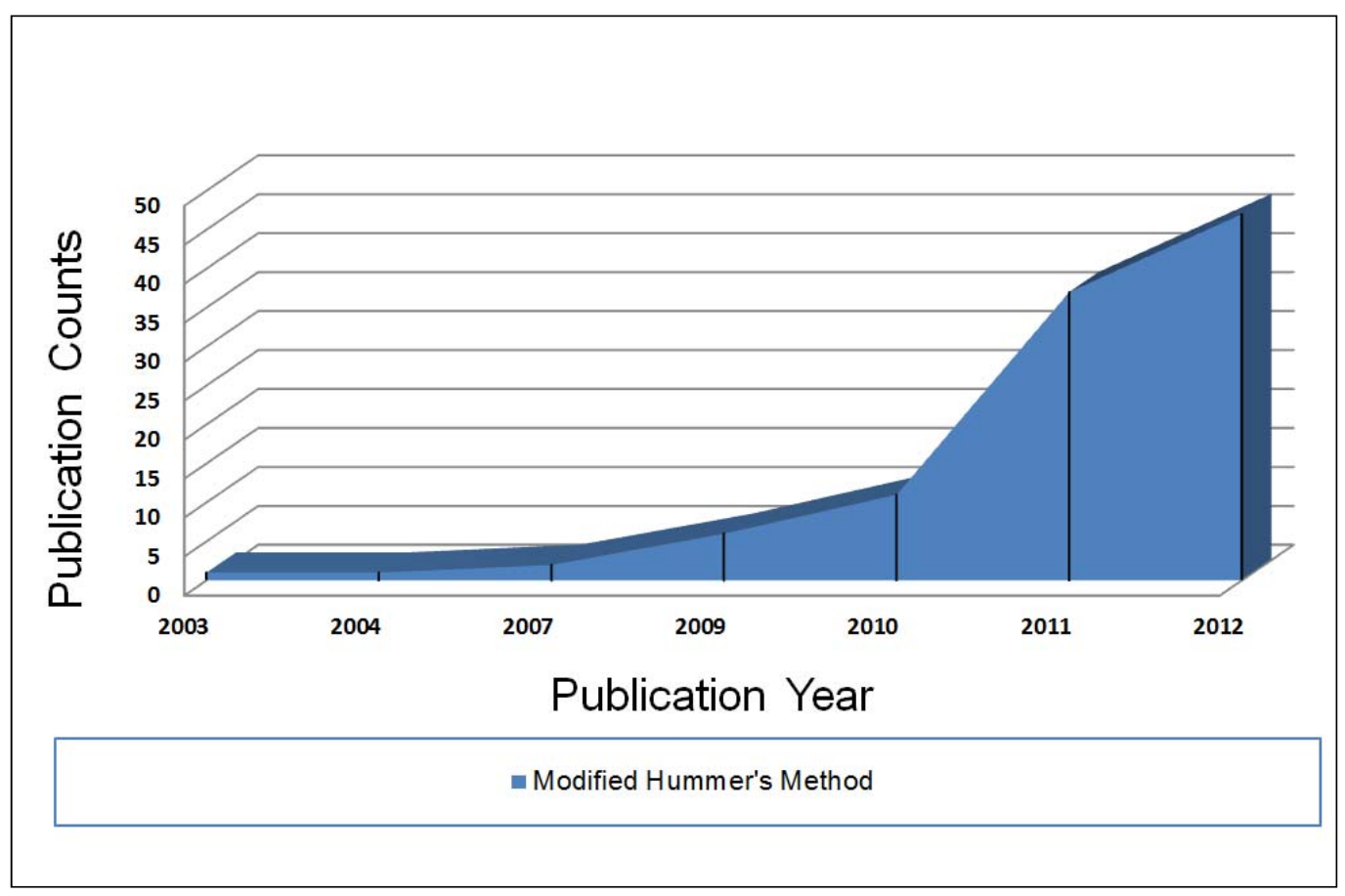

Figure 7: Number of publications on modified Hummer's method over past 20 years. [Source: Web of Science, ISI] 
Optimizations had been made to the Hummer's method and one of the wellcited modified Hummer's synthesis involved removal of sodium nitrate and using more quantity of potassium permanganate or adding phosphoric acid to increase the efficiency of oxidation ${ }^{85}$. The main advantage of this method was that it did not produce toxic gases such as $\mathrm{NO}_{2}$ and $\mathrm{N}_{2} \mathrm{O}_{4}$ that was associated with the original Hummer's method.

In 1999, Kovtyukhova et al ${ }^{86}$ reported that an additional chemical pre-oxidation step before the Hummer's method, using concentrated sulphuric acid $\left(\mathrm{H}_{2} \mathrm{SO}_{4}\right)$, phosphorus pentoxide $\left(\mathrm{P}_{2} \mathrm{O}_{5}\right)$ and potassium persulfate $\left(\mathrm{K}_{2} \mathrm{~S}_{2} \mathrm{O}_{8}\right)$, could result in a complete exfoliation of the graphite and yielded large-sized mono-layered GO.

Then in 2009 , Su et al. ${ }^{13}$ used ultra-sonication to substitute the chemical preoxidation step and this resulted in large flakes with high mobility values up to $12 \mathrm{~cm}^{2} /$ Vs. With larger flakes, the carrier mobility and the electrical conductivity ${ }^{87}$ was increased while maintaining high optical transparencies ${ }^{39}$.

It was noted that although pre-oxidation steps had been commonly used in synthesis of GO, the role and mechanism of pre-oxidation on graphite had yet to be determined. Adding a pre-oxidation step to the Hummer's method, was reported to enlarge the size of GO from similar precursor ${ }^{13}{ }^{86}$. Table 2 summarized notable large GO flakes obtained from chemical oxidation. The largest flakes were obtained from large precursor with the additional preoxidation step. 
Table 2 Comparison of size and hole mobility of GO synthesized from chemical approach from graphite (arranged according to average and maximum size of obtained flake).

\begin{tabular}{|c|c|c|c|c|c|}
\hline Ref & Method & Starting Material & Lateral area/size & Thickness & Hole mobility \\
\hline 13 & $\begin{array}{l}\text { MH (With } \\
\text { ultrasonic pre- } \\
\text { oxidation) }\end{array}$ & 3-5mm graphite & $\begin{array}{l}\text { Up to } 1000 \mu \mathrm{m} \\
\text { in lateral size }\end{array}$ & $\begin{array}{l}\text { Monolayer, } \\
\quad \sim 1 \mathrm{~nm}\end{array}$ & $\begin{array}{c}800^{\circ} \mathrm{C} \text { thermal } \\
4-12 \mathrm{~cm}^{2} / \mathrm{Vs}\end{array}$ \\
\hline 88 & $\begin{array}{c}\text { Graphite } \\
\text { intercalation, } \\
\text { followed by } \mathrm{MH}\end{array}$ & $<800 \mu \mathrm{m}$ graphite & $\begin{array}{l}\text { Up to } 100 \mu \mathrm{m} \text { in } \\
\text { lateral size }\end{array}$ & $\begin{array}{l}\text { Monolayer } \\
\qquad 1 \mathrm{~nm}\end{array}$ & - \\
\hline 89 & $\begin{array}{l}\text { MH (With } \\
\text { ultrasonic pre- } \\
\text { oxidation) }\end{array}$ & $3-5 \mathrm{~mm}$ graphite & $\begin{array}{c}\text { Up to } 50 \mu \mathrm{m} \text { in } \\
\text { lateral size }\end{array}$ & $\begin{array}{l}\text { Monolayer } \\
\quad \sim 1 \mathrm{~nm}\end{array}$ & $\begin{array}{c}1000^{\circ} \mathrm{C} \\
\text { thermal } \\
3.5 \mathrm{~cm}^{2} / \mathrm{Vs}\end{array}$ \\
\hline 85 & $\mathrm{MH}$ & $\sim 150 \mu \mathrm{m}$ graphite & $\begin{array}{l}\text { Estimated up to } \\
50 \mu \mathrm{m} \text { in lateral } \\
\text { size }\end{array}$ & $\begin{array}{l}\text { Monolayer, } \\
\sim 1.1 \mathrm{~nm}\end{array}$ & - \\
\hline 90 & $\begin{array}{l}\text { MH (With } \\
\text { chemical pre- } \\
\text { oxidation) }\end{array}$ & $\begin{array}{c}<0.044 \mathrm{~mm} \text { mesh } \\
\text { graphite }\end{array}$ & Up to $800 \mu \mathrm{m}^{2}$ & $\begin{array}{c}\text { Monolayer, } \\
\text { 0.6nm } \\
\text { (Reduced } \\
\text { layer) }\end{array}$ & - \\
\hline 91 & $\mathrm{HM}$ & $<0.1 \mathrm{~mm}$ graphite & $\mathrm{Up}$ to $5 \mu \mathrm{m}^{2}$ & $\begin{array}{l}\text { Monolayer, } \\
1.0-1.4 \mathrm{~nm}\end{array}$ & - \\
\hline 86 & $\begin{array}{c}\text { MH (With } \\
\text { chemical pre- } \\
\text { oxidation) }\end{array}$ & $\begin{array}{c}<0.044 \mathrm{~mm} \text { mesh } \\
\text { graphite }\end{array}$ & $\mathrm{Up}$ to $0.8 \mu \mathrm{m}^{2}$ & $\begin{array}{l}\text { Monolayer, } \\
1.1-1.4 \mathrm{~nm}\end{array}$ & - \\
\hline
\end{tabular}

MH: Modified Hummer's method HM: Hummer's method 


\subsection{StRuCTURE \& PROPERTIES OF REDUCED GRAPHENE OXIDE}

Most applications of $\mathrm{GO}$ required a reduction step to restore its electrical conductivity. However, some applications such as electrochemical sensing, the GO was used as synthesized ${ }^{92}$. During a reduction process, some of the $\mathrm{sp}^{3}$ structure in the GO was converted back into $\mathrm{sp}^{2}$ structure. This reduction process could be varied in strength, duration and method to tune the resultant electrical, structure and chemical properties of the resultant reduced GO (rGO).

However, even after a long duration of chemical reduction, rGO still could not achieve a complete $\mathrm{sp}^{2}$ structure, from a $\mathrm{sp}^{3}$ structure, comparable to that of pristine graphene. This limited conversion was because of the presence of Stone-wales defects and holes (due to loss of carbon and remnant oxygen atoms). Figure 8 showed possible oxygen functional groups that were found on rGO.

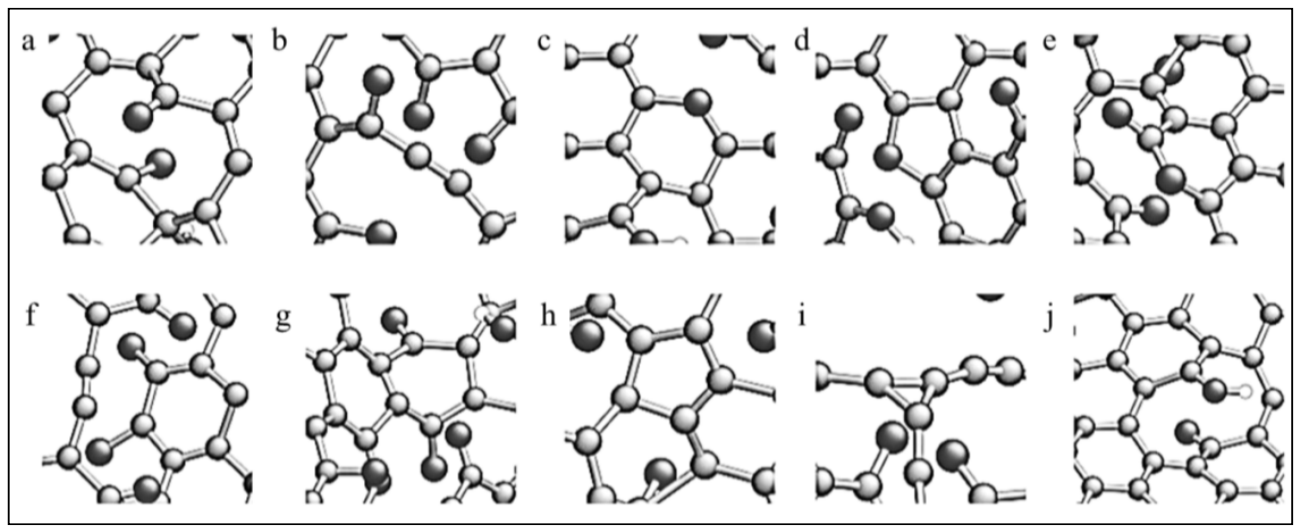

Figure 8: Possible oxygen functional groups and carbon arrangements formed after annealing (a) pair of carbonyls, (b) carbon chain, (c) pyran, (d) furan, (e) pyrone, (f) 1,2quinone, (g) 1,4-quinone (h) five-member carbon ring (i) three-member carbon ring (j) phenol. The darkest shade was oxygen, followed by carbon and no shading in hydrogen atoms. Adapted from ref. ${ }^{93}$

As the reduction of GO proceeds, its electrical resistance would exhibit a power law decay ${ }^{94}$. Thus by varying the degree of reduction, the electrical on-off ratios and properties of $\mathrm{r}$ GO could be tuned for different applications ${ }^{43}$ (Figure 9). A 
mildly-reduced sheet exhibited a high on-off ratio with its transport dominated by voltage-dependent carrier tunnelling or hopping between $\mathrm{sp}^{2}$ clusters ${ }^{95}$ and low electrical conductivity. As reduction of GO proceeded, the size of the $\mathrm{sp}^{2}$ domains did not increase after the removal of oxygen in chemical reduction. Instead, oxygen moieties were removed and some of the $\pi$-bonds in the carbon were restored thus providing percolation pathways between the 2-3 $\mathrm{nm} \mathrm{sp}^{2}$ domains already present in the GO. A heavily-reduced sheet would exhibit a low on-off ratio with ambi-polar characteristics and high electrical conductivity.

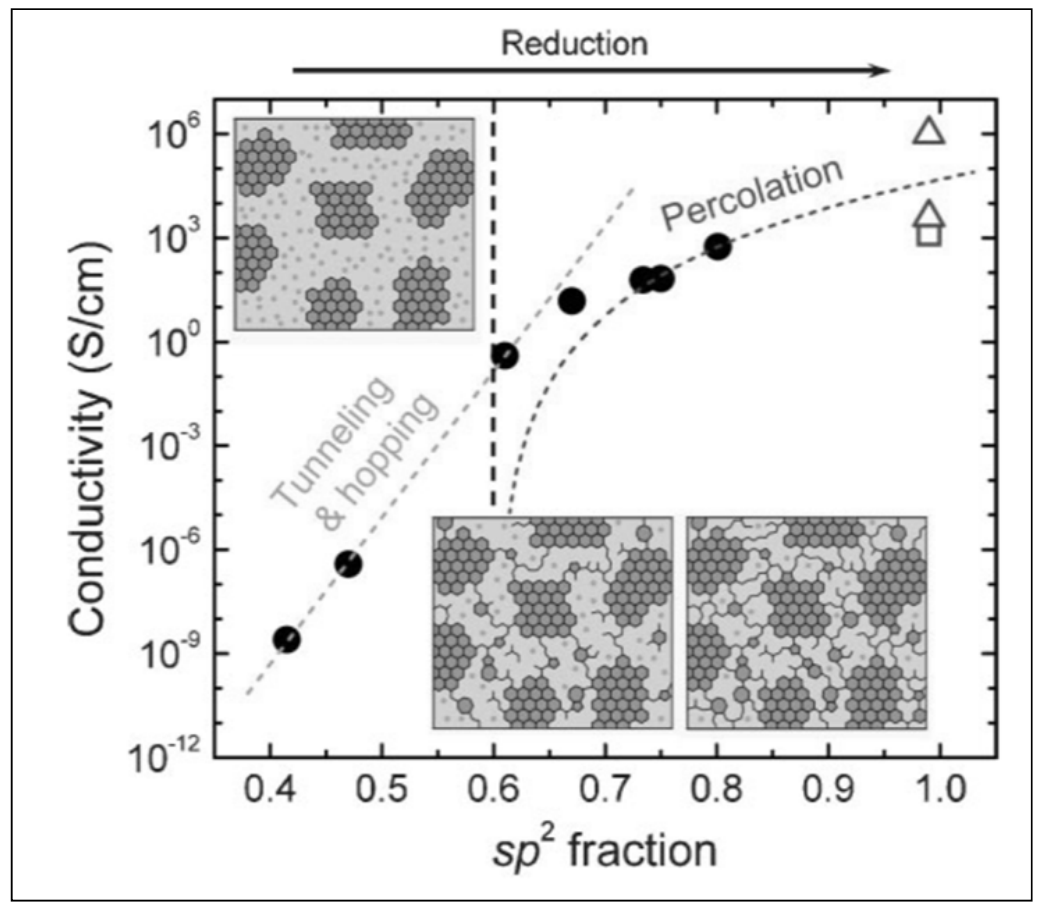

Figure 9: Conductivity of thermally reduced GO as a function of sp2 fraction from XPS. Experimental data fitting reveals two different regimes for electrical transport. Tunneling/hopping dominate transport at $\mathrm{sp}^{2}$ fractions below 0.6 while percolation dominated above. Three references were given as comparison; values of graphene with minimum conductivity (lower triangle), doped conductivity of ideal graphene (upper triangle) and polycrystalline graphite (square). Insert shows evolution of GO structural model at different stage of reduction. Darker areas represent $\mathrm{sp}^{2}$ clusters and lighter areas represents $\mathrm{sp}^{3}$ matrix ${ }^{96}$

The electrical conductance in rGO was finite even after a heavy reduction process due to the fixed $\mathrm{sp}^{2}$ domain size and the limited number of possible conduction pathways using most reducing methods ${ }^{95}$. 


\subsection{METHODS IN REDUCED GRAPHENE OXIDE SYNTHESIS}

The earliest known example of reduced GO was reported by Brodie ${ }^{14}$ in 1859 . He reported that the oxygen content in graphite peaked after four successive oxidative treatments and the net molecular formula was $\mathrm{C}_{2.19} \mathrm{H}_{0.80} \mathrm{O}_{1.00}$. Thereafter, he heated the product at $220^{\circ} \mathrm{C}$ and the composition changed to $\mathrm{C}_{5.51} \mathrm{H}_{0.48} \mathrm{O}_{1.00}$. Since then, much research had been done on reducing the oxygen moieties on GO to restore the electrically conductive $\mathrm{sp}^{2}$ hybridization and graphene-like structure ${ }^{15}$. A summary of the various synthesis methods to reduced GO was shown in Figure 10.

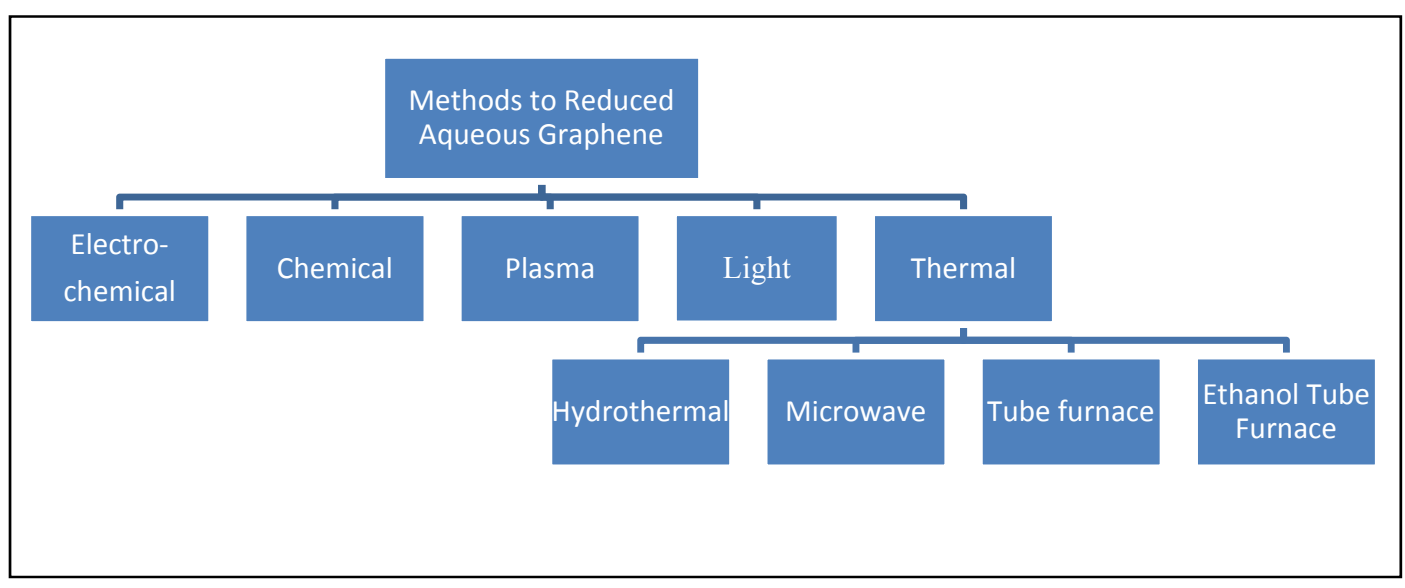

Figure 10: Methods to reduced GO

\subsubsection{ELECTROCHEMICAL}

Electrochemical method of reducing GO had produced end products of $\mathrm{C} / \mathrm{O}$ ratio of $\sim 1: 14^{97}$. Although this $\mathrm{C} / \mathrm{O}$ ratio was significantly lower and the method was not efficient compared to the other methods, it had the advantage of roomtemperature operations and avoided usage of toxic reagents (e.g. hydrazine and sodium borohydride). The electrochemical reduction could be performed simply by scanning a GO-coated glassy carbon electrode in $\mathrm{NaCl}$ aqueous solution with potential from 0.7 to $-1.1 \mathrm{~V}$. In which during the first cycle scanned an irreversible reduction peak, which was assigned to the reduction of the oxygen moieties on GO, could be observed at $-0.87 \mathrm{~V}^{98}$. 
GO could also be electrophoretically deposited on a positive electrode with $10 \mathrm{~V}$ of applied voltage in less than 30 second as an alternative to coating or using chemicals to attach the GO to the electrode ${ }^{99}$. This deposition also resulted in a simultaneous reduction of the GO.

\subsubsection{CHEMICAL}

Many chemicals could be used to reduced GO. They include hydrazine ${ }^{39,100,}$ alcohols $^{101}$, sodium borohydride ${ }^{102}$, hydriodic acid $^{100}$, strong alkaline ${ }^{103}$, metal powders $^{104}$ and sulfonate-containing ${ }^{105}$ compounds. The advantages of using chemical reducing method was that it was aqueous processable and possible to functionalize or dope the GO simultaneously.

Hydrazine was the most commonly used chemical reducing agent for GO and it can produce highly reduced GO at low temperatures ${ }^{39}$. However, as with most reducing chemical agents, particularly hydrazine was highly toxic. Therefore, care and use must be minimized. It was reported that the use of hydrazine vapor reduction instead of liquid hydrazine was sufficient to reduce GO. A small quantity of hydrazine could be placed in a sealed container with the GO coated sample and heated to about $70^{\circ} \mathrm{C}$ for 16 hours to obtain reduced $\mathrm{GO}^{39,106}$. The formation of unsaturated and conjugated carbon atoms after hydrazine treatment helped restore the electrical conductivity in hydrazine-processed $\mathrm{GO}^{40}$ (Figure 11). Reduced GO could also be dispersed in hydrazine, after an in-situ reduction; the $\mathrm{N}_{2} \mathrm{H}_{4}{ }^{+}$counter-ions surrounded the negatively charged reduced GO sheets and prevented aggregations for months ${ }^{107}$.

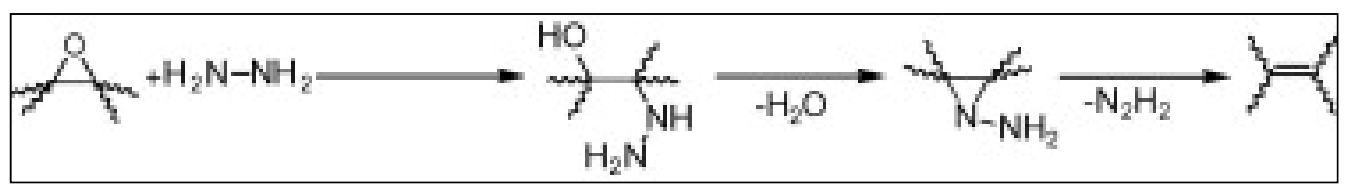

Figure 11: A proposed reaction pathway for epoxide reduction with hydrazine ${ }^{40}$ 
However, the reduction of GO resulted in the incorporation of heteroatomic impurities into the structure, due to hydrazine's reaction with anhydrides \& lactones to form hydrazides and in quinones to form hydrazones ${ }^{40}$. These C-N bonds formed causes the reduced GO to be n-doped after the reduction.

In contrast to the $\mathrm{C}-\mathrm{N}$ residual bonds left by hydrazine reduction, heteroatomic $\mathrm{C}-\mathrm{OH}$ hydroxyl groups were left by sodium borohydride reduction ${ }^{108}$. Thus sodium borohydride demonstrated a more effective reduction than hydrazine. In the same study, although a comparable C-O composition was observed between the two sheets; the sheet resistances of sodium borohydride-reduced films $(59 \mathrm{k} \Omega / \square)$ were much lower than hydrazine-reduced films $(780 \mathrm{k} \Omega / \square)^{102}$. The lower electrical conductivity in hydrazine-reduced films was attributed to the addition of nitrogen atoms into the structure which acted as a donor compensating the p-type holes in reduced $\mathrm{GO}^{1,102}$.

\subsubsection{PLASMA}

A single sheet GO could reached a conductivity plateau after 5 seconds of RFgenerated hydrogen plasma $(30 \mathrm{~W} \text { at } 0.8 \mathrm{mbar} \text {, room temperature })^{109}$. GO could also be reduced using e-beam generated plasma produced in methane/argon mixtures $(-2 \mathrm{kV}, 150 \mathrm{G}$ magnetic field at 90mTorr). Addition of methane (2\%) into this e-beam generated plasma caused a sharp decrease in the oxygen concentration from 37 at. $\%$ to 8 at. $\%$ after 30 seconds $^{110}$. The transport in both optimally hydrogen plasma-reduced and hydrazine-reduced GO occurred similarly via variable range hopping between localized $\mathrm{sp}^{2}$ states in $\mathrm{sp}^{3}$ matrix ${ }^{45}$, 111. As with chemical reduction, plasma reduction could also add heteroatomic impurities to the reduced GO. By using 1 hour of nitrogen plasma $(500 \mathrm{w}$ at 1Torr, room temperature), the addition of nitrogen was quantified by XPS to be 5.8at. \%, thus n-doping the reduced $\mathrm{GO}^{112}$. Using an optimal 3 minutes argon plasma treatment on GO film yielded a smooth and conductive sample (7.4 $\mathrm{S} / \mathrm{cm}$ ) in contrast to a over-treatment of a 5 minutes treatment which yielded a degraded surface morphology from ion bombardment and a lower conductivity $(5.1 \mathrm{~S} / \mathrm{cm})^{113}$. 
Although the process needed to be optimized properly and controlled precisely before reduction of GO was efficient, this process was potentially highly compatible with existing semiconductor industries. Plasma etching was already a conventional technology in the semiconductor industry and existing plasmaenhanced chemical vapor deposition chamber could be directly used to provide the hydrogen plasma for reduction. Moreover, the reduction process could be monitored via the end-point detectors on the equipment ${ }^{114}$. Thus plasma reduction method looked very promising for scalable aqueous grapheme reduction.

\subsubsection{LIGHT}

GO is a soft matter and can be reduced under light irradiation ${ }^{115-118}$. The photo energy provides the thermal energy needed for heating the GO which induce deoxygenating reactions ${ }^{115}$. The degree of GO reduction is wavebanddependent. The O:C ratio can be reduced to $39 \%$ and $28 \%$ using $400-800 \mathrm{~nm}$ and 350-800nm irradiation respectively from initial 47\%, while using 520$800 \mathrm{~nm}$ irradiation resulted in negligible change ${ }^{119}$. UV-irradiation reduction method could also be used to precisely reduce selected areas of GO via photolithography ${ }^{120}$, multi-beam interference lithography ${ }^{121}$ and focused laser beam $^{122}$.

Electrical conductivity can be achieved by using a combination of light and chemical or photo-chemical method ${ }^{123,124}$. Using UV-irradiation on GO solution with $\mathrm{TiO}_{2}$ suspensions, a stable solution of rGO can be obtained as the interaction between $\mathrm{TiO}_{2}$ particles and the $\mathrm{GO}$ sheets prevent the agglomeration of the subsequent reduced $\mathrm{GO}^{125}$.

\subsubsection{THERMAL}

\section{Hydrothermal}

Hydrothermal reduction of GO required just an autoclave and produced uncontaminated reduced GO (as compared to hydrazine ${ }^{39}$ or sulfonate ${ }^{105}$ chemical reduction). During hydrothermal reduction, the molar ratio of $\mathrm{sp}^{2}$ to $\mathrm{sp}^{3}$ carbon could increase from 1.8 to $5.6^{35}$, which was comparable to 12 hours 
hydrazine vapor reduction ${ }^{5}$. The high temperature $\left(180^{\circ} \mathrm{C}\right)$ and pressure during the hydrothermal reaction also recovered the aromatic structures by repairing defects as observed in a decrease of Raman $\mathrm{I}_{\mathrm{D}} / \mathrm{I}_{\mathrm{G}}$ ratio from 0.96 to 0.90 after a 6-hour hydrothermal reduction, as compared to an increase of 1.44 after hydrazine chemical reduction ${ }^{35}$. A one-step hydrothermal reaction, loaded with suitable precursors, could also be used to simultaneously synthesize nanoparticles such as $\mathrm{TiO}_{2}$ on the reduced $\mathrm{GO}^{126}$.

\section{Microwave}

GO could be reduced in the aqueous state using microwave irradiation with the additional of chemical reductant such as ascorbic acid $(0.5 \text { hour })^{127}$ and hydrazine $(1000 \mathrm{~W}, 60 \text { seconds })^{128}$. The type of solvent in which the GO was dispersed in had little effect on the microwave reduction efficiency ${ }^{129}$. GO powders could also be reduced in the dried solid state using microwave irradiation from a commercial microwave oven (700W, within 60 seconds) under ambient conditions ${ }^{130}$. The absence of water in the precursor GO allowed a stronger and more efficient reduction. GO powder precursor dried at $120^{\circ} \mathrm{C}$ before the microwave reduction resulted in an explosive-like exfoliation reaction and more efficient reduction with a higher 2D band FWMH of $600 \mathrm{~cm}^{-1}$ compared to precursors dried at room temperature with a $2 \mathrm{D}$ band of $570 \mathrm{~cm}^{-1}$ only $^{131}$.

The $\pi-\pi$ conjugated region was vital for the transformation of microwave energy to heat and caused the avalanche-like de-oxygenation process reaction; the microwaves caused superheating of the graphene and this heat could be quickly dissipated via the $\pi$ electrons to an GO region contribution to its deoxygenation $^{132}$. The $\mathrm{C} / \mathrm{O}$ ratio was increased from 0.79 to 2.75 after a microwave-reduction of dried GO powders ${ }^{130}$ and the resultant microwavereduced GO consisted of crumpled, few-layer thick sheets ${ }^{128,130}$.

By controlling the temperature $\left(165^{\circ} \mathrm{C}\right)$ of microwave reduction, the method also allowed direct reduction of GO flake coated on polydimethylsiloxane (PDMS) polymer substrates ${ }^{133}$. Pd, $\mathrm{Cu}$ and $\mathrm{CuPd}$ metal nanoparticles could also 
be simultaneous reduced and dispersed on the reduced GO by reduction of its metal salt solution with GO using hydrazine under microwave irradiation ${ }^{128}$.

\section{Tube Furnace}

The thermal reduction in ultra-high vacuum (UHV) was more efficient compared to argon atmosphere, hydrogen/argon atmosphere or using the hydrazine-vapor chemical treatment (Table 3 ). With a $700^{\circ} \mathrm{C}$ ultra-high vacuum (UHV) tube-furnace heat treatment, the XPS $\mathrm{C} 1 \mathrm{~s} / \mathrm{O} 1 \mathrm{~s}$ ratio of $\mathrm{GO}$ was increased from 2.8 to $13.2^{106}$. Using the empirical Tuinstra-Koenig relation, the $\mathrm{sp}^{2}$ graphitic domain size on the reduced GO was observed to increase from $\sim 2.5 \mathrm{~nm}$ to $\sim 2.8 \mathrm{~nm}$ after heat treatment of above $1100{ }^{\circ} \mathrm{C}^{96}$. This self-healing of the carbon lattice vacancies in GO during becomes less effective with increasing number of initial vacancies ${ }^{134}$. 
Table 3 The values of $\mathrm{C1s} / \mathrm{O1s}$ (atomic ratios) of thermal-reduced and hydrazine-reduced GO samples obtained from XPS survey spectra ${ }^{106}$

\begin{tabular}{|c|c|c|c|c|c|c|}
\hline Conditions & - & $200{ }^{\circ} \mathrm{C}$ & $500{ }^{\circ} \mathrm{C}$ & $700{ }^{\circ} \mathrm{C}$ & $900{ }^{\circ} \mathrm{C}$ & $1000^{\circ} \mathrm{C}$ \\
\hline As-deposited film & $\begin{array}{c}2.8 \pm \\
0.1\end{array}$ & - & - & - & - & - \\
\hline Heat treatment in $\mathrm{Ar}$ & - & $\begin{array}{c}3.9 \pm \\
0.1\end{array}$ & $\begin{array}{l}6.8 \pm \\
0.2\end{array}$ & - & - & $\begin{array}{c}11.36 \pm \\
0.3\end{array}$ \\
\hline $\begin{array}{c}\text { Heat treatment in Ar } \\
\text { and } \mathrm{H} 2\end{array}$ & - & $\begin{array}{c}3.9 \pm \\
0.1\end{array}$ & $\begin{array}{l}7.3 \pm \\
0.2\end{array}$ & - & $\begin{array}{c}14.1 \pm \\
0.3\end{array}$ & $12.4 \pm 0.3$ \\
\hline UHV heat treatment & - & - & $\begin{array}{c}8.9 \pm \\
0.2\end{array}$ & $\begin{array}{c}13.2 \pm \\
0.3\end{array}$ & - & - \\
\hline Hydrazine treatment & $\begin{array}{c}8.8 \pm \\
0.2\end{array}$ & - & - & - & - & - \\
\hline
\end{tabular}

UHV: Ultra-high vacuum

The epoxy coverage on the precursor GO, the substrate which the GO was attached on and the gas precursors used during the thermal reduction could alter the properties of the final reduced GO. The lattice damage of reduced GO after a thermal reduction was proportional to the pre-existing surface density of epoxy species. At low epoxy coverage, the epoxy undergo reversible desorption while at higher coverage, it became the precursor for release of $\mathrm{CO}$ and $\mathrm{CO}_{2}$ gases thus leading to lattice damage ${ }^{135}$. It was also reported that vacuum annealing at $500^{\circ} \mathrm{C}$ decreased the electrical resistivity of bilayer GO but not of monolayers, suggesting that substrate interactions, such as substrate surface roughness or APTES, could restrict the thermal healing process on layer closest to substrate ${ }^{109}$. Also, by simply adding ammonia $\left(\mathrm{NH}_{3}\right)$ into the thermal 
annealing process, n-doped reduced GO with $\mathrm{C}-\mathrm{N}$ bonds was obtained in the carbon structure ${ }^{136}$, thus altering the properties of reduced GO.

\section{Ethanol Tube Furnace}

Carbon loss as $\mathrm{CO}_{2}$ and $\mathrm{CO}$ gases were observed during thermal reduction of GO in UHV starting from $70^{\circ} \mathrm{C}^{137}$ resulting in vacancies and topological defects in the reduced $\mathrm{GO}^{138}$. Thus the addition of other carbon-containing reactants could help GO to regain the electrical and mechanical properties of pristine graphene ${ }^{137}$. Using carbon sources at elevated temperatures and atmospheric pressure, similar to CVD growth of carbon nanotubes setup, resulted in a more effective reduction of GO which was attributed to a recovery in graphitic structure $^{12}$. An enlargement of the $\mathrm{sp}^{2}$ domain size, from 1 to $4 \mathrm{~nm}$, was observed and this translated to higher electrical conductivity and carrier mobility compared to hydrazine reduced or thermally reduced samples ${ }^{12}$. This enlargement could be attributed to the thermal energy favouring the clustering of the $\mathrm{sp}^{2}$ phase ${ }^{139}$. Further studies on the ethanol CVD graphitization of GO was needed to elucidate its mechanism. Thereafter, in the next two sections, the importance of the analyte-of-interest in biosensing - sodium and Inerleukin-6 (IL-6) was presented. 


\subsection{SODIUM IN SWEAT}

The human eccrine sweat gland consisted of two distinct regions, the secretory coil and the reabsorptive duct. Sweat was produced in the secretory coil by the electrogenic of $\mathrm{NaCl}$ and osmosis of water, while the reabsorptive duct reabsorbs $\mathrm{Na}^{+}$via an ouabain-sensitive $\mathrm{Na}^{+}-\mathrm{K}^{+}$ATPase localized on the basolateral membrane of ductal cells ${ }^{140}$. The activity of the sweat gland occurred as a result of a central neural drive via discharge of cholinergic fibres, which was controlled by the thermoregulatory centre found in the hypothalamus portion of the brain.

The hypothalamus portion of the brain sent efferent signals to the intermediolateral cell column of the spinal $\operatorname{cord}^{141}$. The signals then passed through gray communicans and combined with peripheral nerves. These nerves fibres terminated at the surrounding the sweat glands ${ }^{142}$ and caused stimulation of the sweat glands.

During physical exercise, such as cycling and running, profuse sweating led to the loss of electrolytes such as sodium and potassium ions ${ }^{143}$. With increasing sweat rate, only $\mathrm{Na}^{+}$and $\mathrm{Cl}^{-}$(from extracellular compartments) concentration increased while $\mathrm{Ca}^{2+}$ (from intracellular space) decreased; $\mathrm{K}^{+}$and $\mathrm{Mg}^{2+}$ (from intracellular space) remains unchanged ${ }^{144}$. The concentration of $\mathrm{Na}^{+}$loss also increased with higher sweat rates (Figure 12). Therefore, in hotter climate or during exercise, the loss of $\mathrm{Na}^{+}$was significant. 


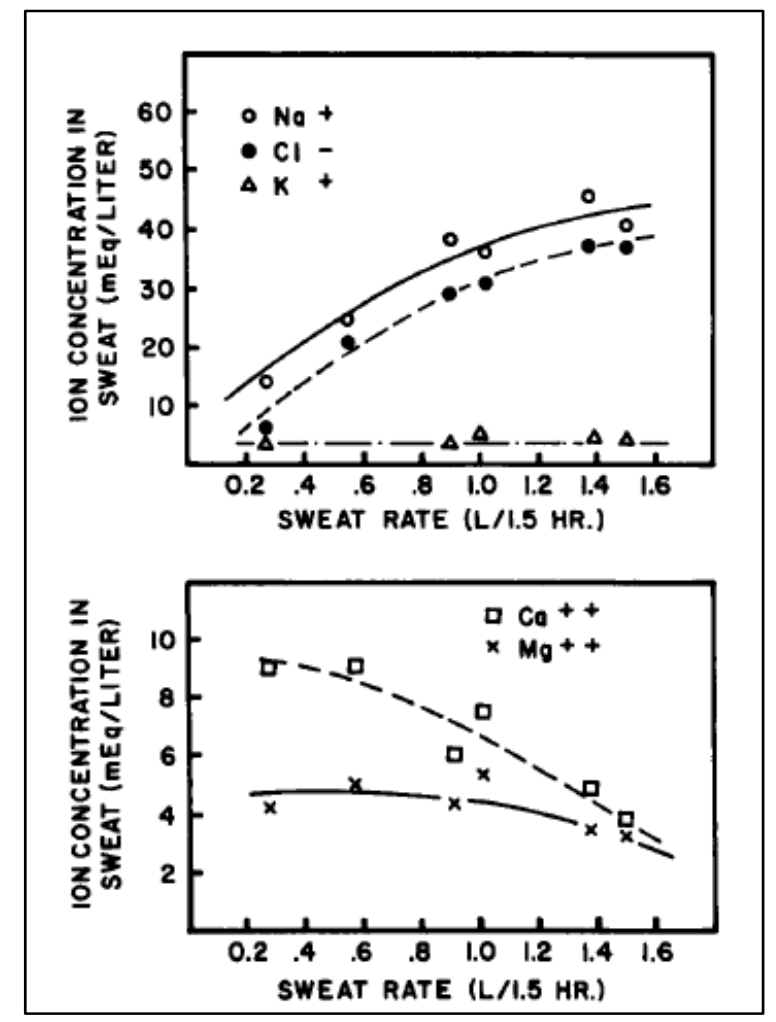

Figure 12: Various salts' ion concentration vs. sweat rates. With increasing sweat rate, $\mathrm{Na}^{+}$and $\mathrm{Cl}^{-}$increased while the other electrolytes decreased or remained the same ${ }^{144}$

Electrolyte loss through urine during exercise was small because of decreased urine formation and increased renal $\mathrm{Na}^{+}$reabsorption. The loss of $\mathrm{Na}^{+}$and $\mathrm{Cl}^{-}$ ions produce deficits of $\sim 5$ and $7 \%$ in the body, while $\mathrm{K}^{+}$and $\mathrm{Mg}^{2+}$ decreased by less than $1.2 \%{ }^{144}$. During exercise, the ionic deficits of major concern were those of $\mathrm{Na}^{+}$and $\mathrm{Cl}^{-}$occurring in sweat. It was reported that measurements of sodium and potassium ions in whole body sweat loss could be used to predict changes in serum ${ }^{143}$ and thus used to dictate rehydration strategy.

In addition, sodium levels in sweat was also used to diagnose cystic fibrosis ${ }^{145}$, 146 and predict exercise-associated hyponatremia ${ }^{147,148}$. Therefore, sodium measurement in sweat was important and essential. The next two subsections will review the two different types of sweat collection technique; how $\mathrm{Na}^{+}$ concentration changes with different factors and finally, types of sodium sensors that were in the market. 


\subsubsection{SWEAT COLLECTION TECHNIQUES}

There were two types of sweat collection techniques - Whole body washdown (WBW) and the regional site collection (RSC). RSC tended to overestimate the sodium and potassium concentration at all points, the forearm, back, chest, forehead and thigh, for both sexes as compared to $\mathrm{WBW}^{143,149}$. However, regression equations could be used to accurately and reliably predict the WBW sweat sodium and potassium concentration from RSCs. $\mathrm{Na}^{+}$from sweat at the RSC from thigh region corresponded best to WBW. This was shown in Figure 13. A comparison of the advantages and disadvantages of WBW vs. RSC was summarized in Table 4.

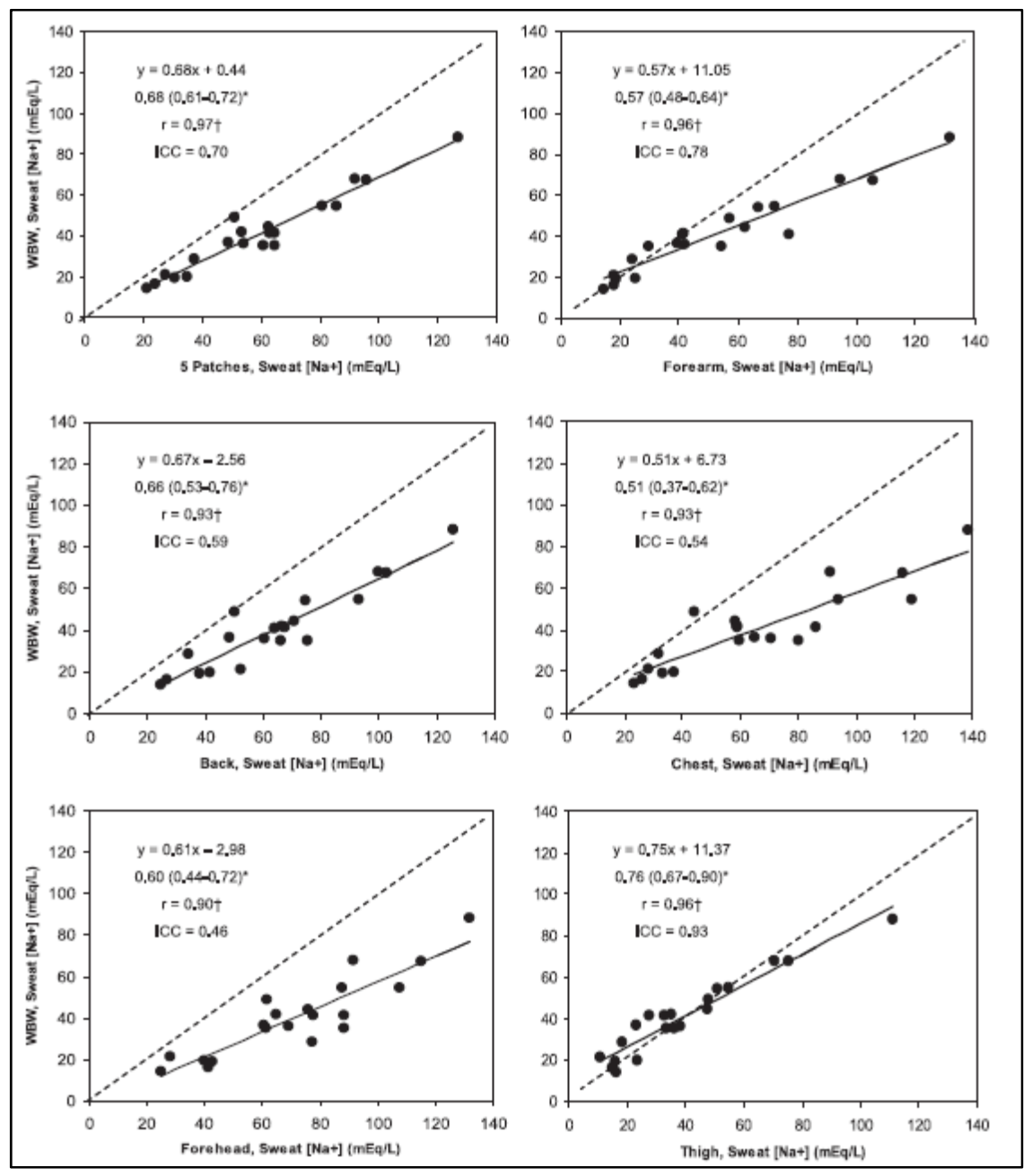

Figure 13: Regression of 5-site and individual RSC sweat $\mathrm{Na}^{+}$concentration. The dashed line was the line of identity. It was observed that the thigh sweat RSC correlated well with WBW value ${ }^{143}$ 


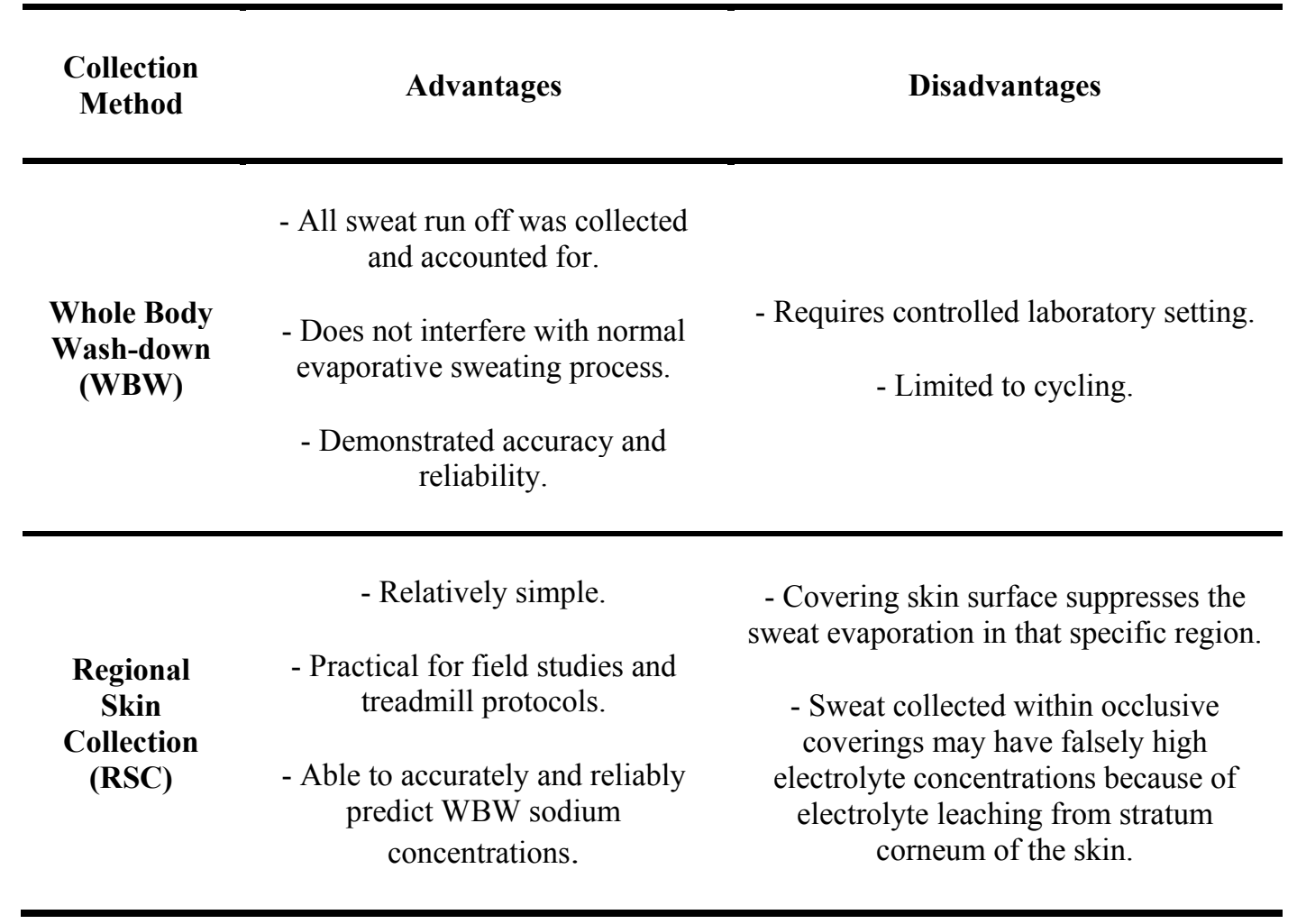

In the RSC, the $\mathrm{Na}^{+}$concentration in sweat sampled on the skin surface was observed to increase because the rate of $\mathrm{Na}^{+}$reabsorption of sweat glands do not increase proportionally as the rate of sweat increases ${ }^{150}$. Using RSC could also result in higher inaccurate electrolyte reading resulting from stratum corneum leaching ${ }^{151,152}$. However, if the skin could be kept dry maybe by wicking material to absorb sweat, it could be possible to obtain reliable estimates of local sweat electrolyte concentrations. A sensor with wicking capability could prevent reabsorption of the $\mathrm{Na}^{+}$back into the body. The sensor would then measure the true $\mathrm{Na}^{+}$excretion. It had been suggested that above a certain sweat rate threshold, there was insufficient time for complete sodium ion reabsorption from the duct ${ }^{153}$. This reabsorption of $\mathrm{Na}^{+}$was greater on the trunk (chest and back) than on the limbs (forearms and thigh) ${ }^{154}$. 


\subsubsection{INFLUENCES TO SODIUM CONCENTRATION IN SWEAT}

Individuals with higher aerobic fitness levels had greater $\mathrm{Na}^{+}$concentration loss in sweat, increased sweat rate and decreased $\mathrm{Na}^{+}$reabsorption rates, during prolonged exercise in the heat $\left(36 \pm 1{ }^{\circ} \mathrm{C}\right)^{155} \cdot \mathrm{Na}^{+}$concentration in sweat increased along with the exercise intensity (Figure 14). The trained group sweat $\mathrm{Na}^{+}$concentration ranged from $45-80 \mathrm{mM}$ while the untrained group ranged from $35-55 \mathrm{mM}$ and this represented a $\sim 22 \mathrm{mM}$ difference between the group.

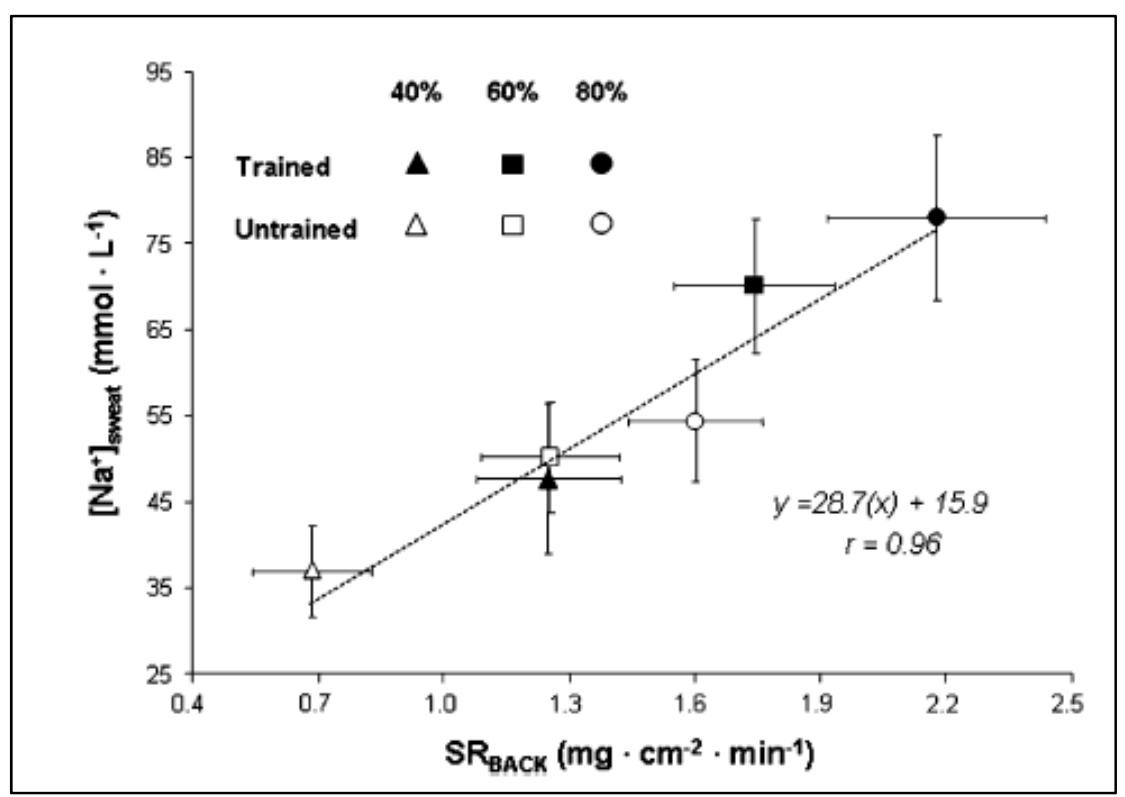

Figure 14: Sweat sodium concentration versus back sweat rate during exercise in heat between untrained and trained groups. Higher aerobic fitness level increased both sweat rate and $\mathrm{Na}^{+}$concentration in sweat ${ }^{155}$

Many studies reported that exercise in heat increased sweat rate and decreased $\mathrm{Na}^{+}$concentration in sweat. The decrease in sodium concentration in heat acclimation was pinpointed to an increase in sodium absorption capacity ${ }^{140}$. A study reported that 10 days of exercise in heat (40C, 45\% humidity) increased the sweat rate by $14 \%$, but decreased the sodium concentration in sweat from $88 \mathrm{mM}$ to $42 \mathrm{mM}^{156}$. The $\mathrm{Na}^{+}$concentration in sweat could decrease dramatically from $90 \mathrm{mM}$ to $60 \mathrm{mM}$ following heat acclimation (Figure 15). 


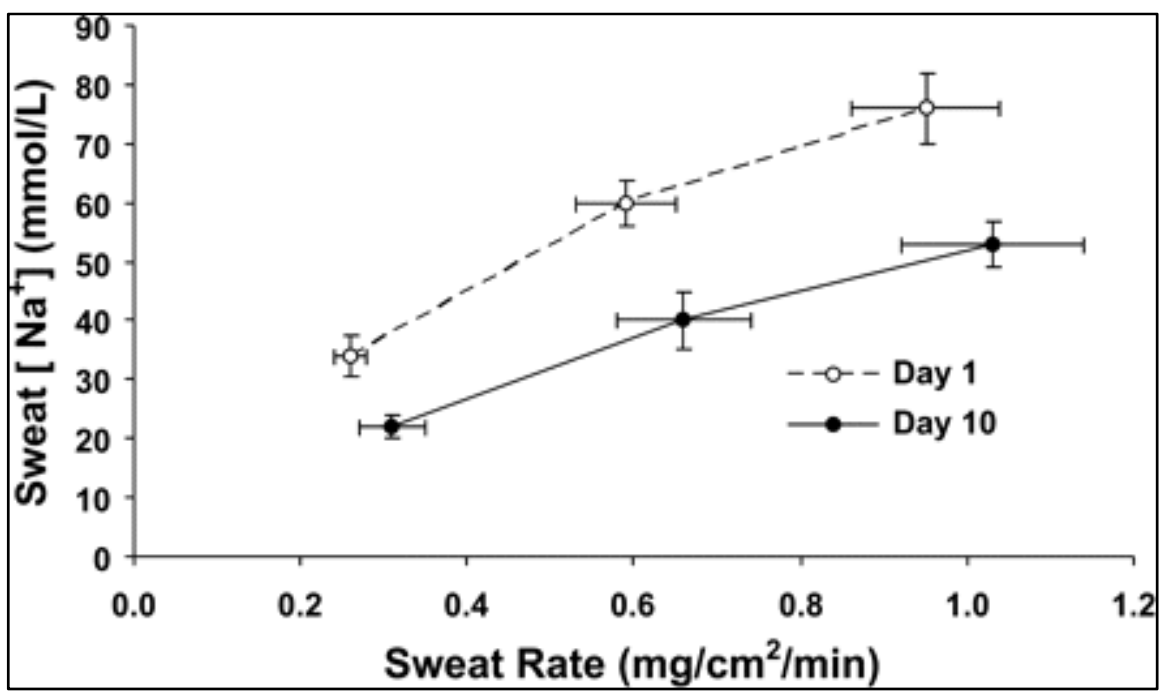

Figure 15: $\mathrm{Na}^{+}$concentration in sweat vs. sweat rate on Day 1 and Day 10 of heat acclimation $^{140}$

It was difficult to make general conclusions on guidelines for fluid and electrolyte intake for teams or groups of players because of the wide ranges of electrolyte loss data observed, thus there was a need for individualized fluid replacement monitoring ${ }^{157}$.

There were some indications that sodium loss was a contributing factor to heatrelated whole body muscle cramps. Sweat sodium was two times higher in individuals with history of heat cramps versus those with none $(54.6 \pm 16.2$ vs. $25.3 \pm 10.0 \mathrm{mM})^{158}$.

High $\mathrm{Na}^{+}$ions in sweat could also indicate cystic fibrosis whereby the patient's eccrine sweat ducts were unable to reabsorb $\mathrm{NaCl}^{145}$. Cystic fibrosis (CF) was the most common inherited disorder of childhood ${ }^{159}$. It was caused by $\Delta \mathrm{F} 508$ mutation in heterologous expression systems ${ }^{160}$ due to misalignment and mislocation of the cystic fibrosis transmembrane conductance regulator $(\mathrm{CFTR})^{161}$. In older $\mathrm{CF}$ subjects, sweat $\mathrm{Na}^{+}$provided the best detection for cystic fibrosis ${ }^{160}$. However, commercial sensors used detection of chloride ions and sweat conductivity for CF diagnosis. 


\subsection{INTERLEUKIN-6 IN CIRCULATION AND IN SWEAT}

Interleukin-6 (IL-6) is a $20-$ to $28-\mathrm{kDa}$ glycoprotein which is secreted by various cells including skeletal muscle cells, epithelial cells, macrophages and lymphocytes. It is a multi-functional cytokine with a wide range of biological activities such as regulation of the immune system, generation of acute phase reactions and also plays a key role in metabolism during exercise ${ }^{162,163}$.

IL-6 had been demonstrated to exert both pro- and anti-inflammatory responses ${ }^{164}$. It was associated with $\mathrm{T}$ cells and macrophages to stimulate immune response in conditions associated with endotoxemia such as thermal injury or other tissue damaged that led to inflammation during infection or after trauma ${ }^{165}$. IL-6 could also act as an anti-inflammatory cytokine, such as increasing neutrophil counts in the blood ${ }^{166}$, production of chemokines and adhesion molecules in lymphocytes ${ }^{167}$.

IL-6 levels in circulation were 1000-fold lower than other secreted cancer biomarkers such as prostate specific antigen (PSA), thus posing a significant analytical challenge ${ }^{168}$. Till date, there had been no commercialization of IL-6 sensors. Enzyme-linked immunosorbent assay (ELISA) and western blot were the staple for detection of IL-6, requiring substantial time, machinery, high cost and specialized manpower training.

IL-6 could bind to plasma membrane receptor complexes containing signal transducing receptor chain gp130 (glycoprotein 130) and the signal transduction involved the activation of JAK (janus kinase), leading to the activation of transcriptions factors of the STAT (signal transducer and activator of transcription). The other major signalling pathway for IL-6 was through the MAPK (mitogen-activated protein kinase), as shown in Figure 16. 


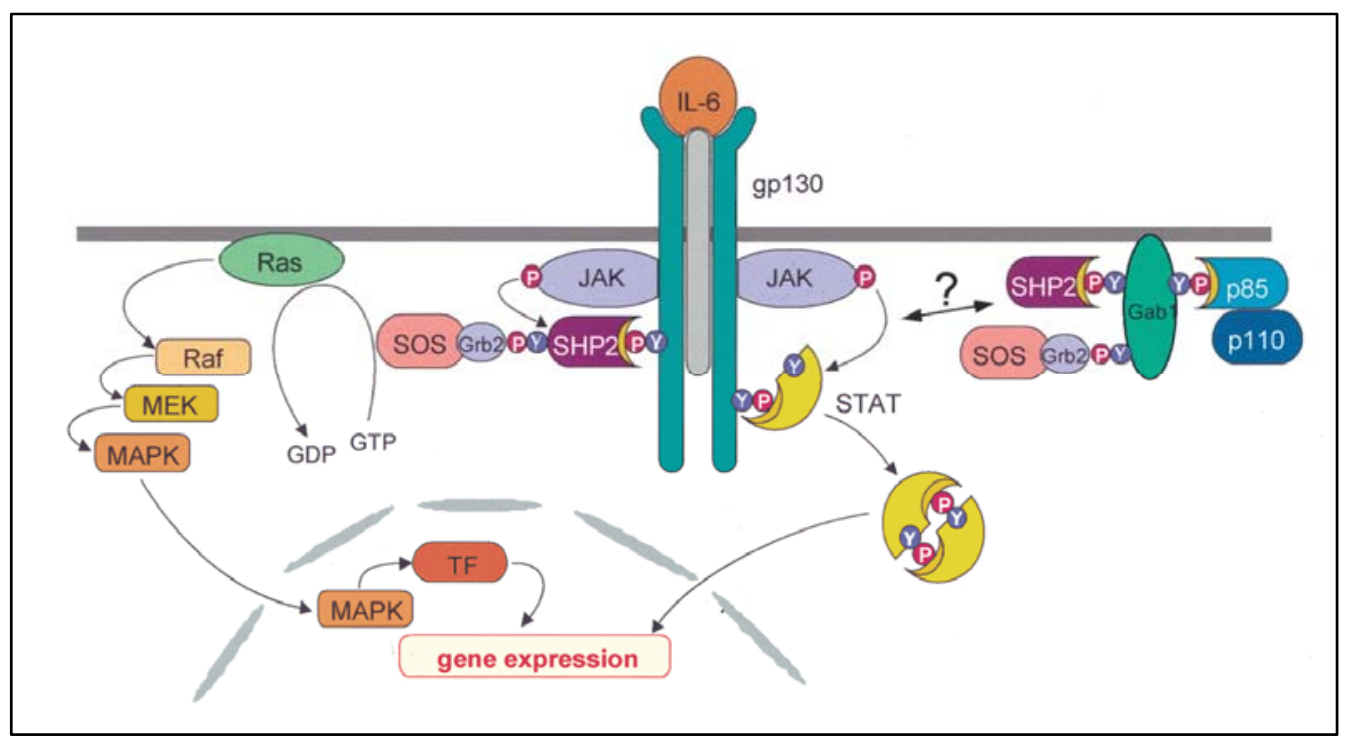

Figure 16: Representation of the JAK/STAT (Janus kinase/Signal transducers and activators of transcription) pathway and the MAPK (mitogen-activated protein kinase) cascade by IL-6 ${ }^{169}$

IL-6 was predominantly produced within the working skeletal muscles during exercise $^{170}$ and its circulation levels increased in response to acute exercise in young males ${ }^{171}$ and could be modulated by acute bout of high-intensity intermittent (HIIT) exercise in comparison to continuous moderate-intensity exercise (MOD) ${ }^{172}$. The IL-6 increase in the circulation during exercise was dramatically more than any other cytokine studied to date with the increase dependent on the duration and intensity of the exercise thus it could be used as an indicator of overtraining in atheletes ${ }^{173}$.

After a strenuous marathon, the level of IL-6 level was increased up to 100-fold and peaked immediately after cessation of exercise, thus serving as an early indicator of glycogen depletion and inflammatory response to training ${ }^{174}$ (Figure 17) . Moreover, the presence and increase of IL-6 from leukocytes following exercise in urine, showed the possibility of non-invasive sensing of cytokines in response to exercise ${ }^{175}$ (Figure 18). 


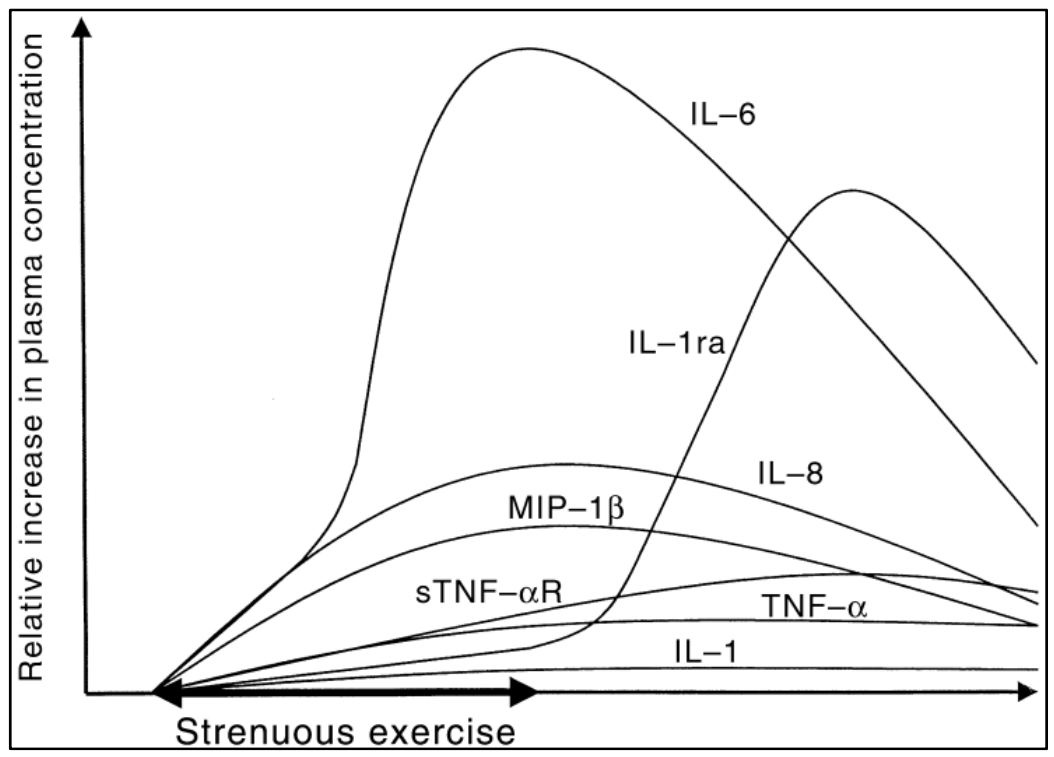

Figure 17: Schematic presentation showing effect of strenuous exercise on the levels of interleukin (IL)-6, interleukin-1 receptor antagonist (IL-1 ra), macrophage inflammatory

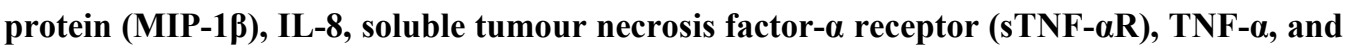
IL-1. The relative increase in plasma concentrations of cytokines during and after exercise was shown $^{174}$

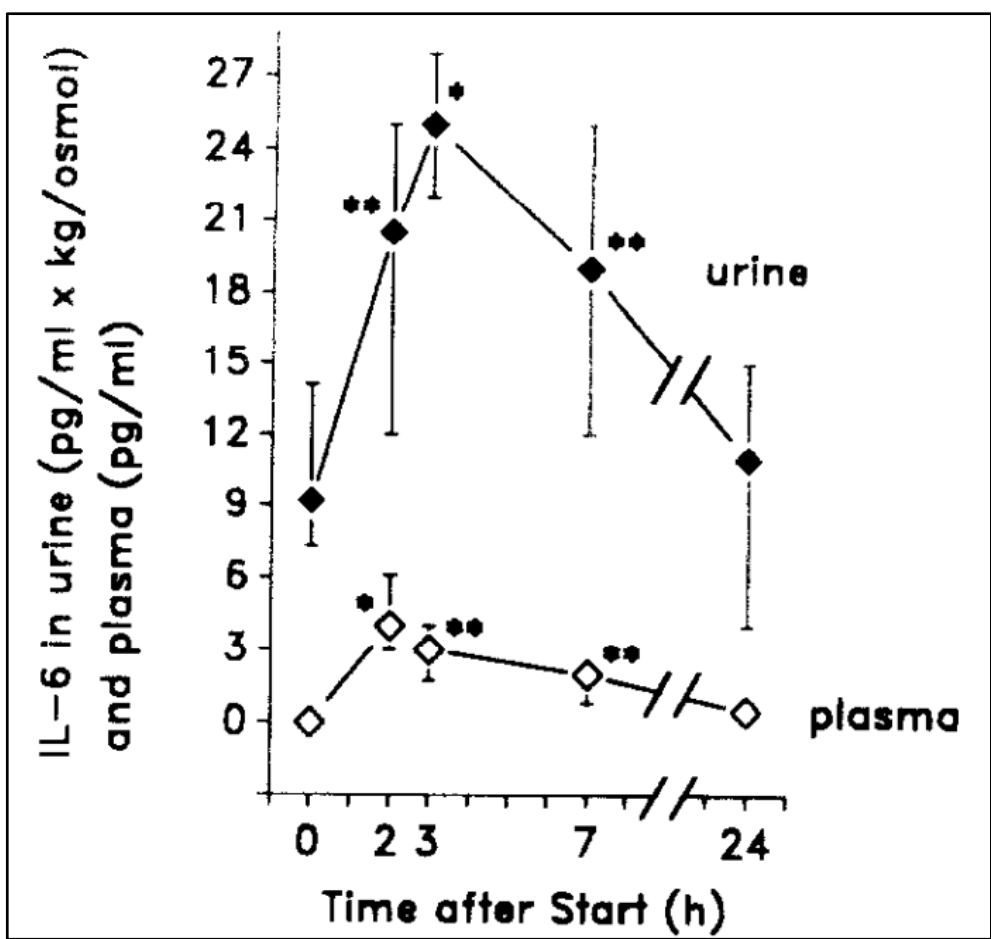

Figure 18: Increase of IL-6 in plasma and urine after running. Both samples were collected before exercise, directly after finishing ( 2 hours) and at indicated time periods thereafter $^{175}$ 
During training, IL-6 could indicate "overtraining syndrome" (OTS) ${ }^{176}$. OTS was described as impaired athletic performance and chronic fatigue which was not remedied by prolonged rest. The aetiology of OTS was unknown as yet. An acute recombinant human IL-6 (rhIL-6) administration to healthy humans induced a heightened sensation of fatigue at rest with depressed mood states and reduced ability to concentrate ${ }^{177}$. It also significantly increased the time taken for a $10-\mathrm{km}$ running trial in trained runners ${ }^{178}$. Chronic elevations in proinflammatory cytokines (especially IL-6) may have detrimental effects on the central nervous system and could account for the many symptoms of OTS ${ }^{179}$. Thus early detection of elevated IL-6 levels may help prevent the onset of OTS.

Since IL-6 levels were inversely related to the muscle glycogen storage, IL-6 may be a useful biomarker for recovery between exercise sessions ${ }^{180}$. Due to its apparent role in glycogen sensing, IL-6 had been termed "muscle energy sensor". Thus it could also be regarded as a carbohydrate sensor during prolonged exercise ${ }^{181}$. It was associated to the amount of skeletal muscle damage ${ }^{182}$ or muscle repair mechanism: whereby macrophages invade into the muscle ${ }^{183}$, as IL-6 level showed a larger increase in eccentric exercise compared to concentric exercise.

Due to the important role of IL-6 in exercise, it was of interest to develop noninvasive methods for measuring IL-6 levels. Currently, IL-6 levels were determined from blood plasma samples, using enzyme-linked immunosorbent assay (ELISA) experiments. This diagnostic test was invasive and required expensive, bulky, highly-specialised equipment. However, non-invasive, pointof-care sampling methods would allow coaches and athletes to tailor training sessions to the athlete's specific recovery and nutritional requirements.

IL- 6 could also be used as a biomarker to monitor health by detecting chronic inflammation which underpinned most chronic diseases such as rheumatoid $\operatorname{arthritis}^{184}$, psoriasis ${ }^{185}$ and tuberculosis ${ }^{186}$. Anti-IL-6 treatment had been approved and highly-successful for the treatment of rheumatoid arthritis ${ }^{187}$. However, during the course of treatment, the IL-6 level had to be monitored as the IL-6 had pro-inflammation benefits as well. 
Previous experiments found no correlation between plasma and salivary IL-6 levels during exercise ${ }^{188}$. Thereafter, studies published in 2006 reported that IL6 was present in sweat and were informative of circulatory levels thus potentially providing a viable and non-invasive method to measure this cytokine $^{189,190}$. However, a proteomic study on sweat found no IL-6 present and that it was unlikely that sweat acts as a plasma transduate ${ }^{191}$. The lack of detection may be associated with the low concentrations. Thus studies must be conducted to determine the presence of IL-6 protein in sweat. After understanding the importance of the two analytes-of-interest, the following section present graphene oxide's role in biosensing.

\subsection{GRAPHENE OXIDE IN BIOSENSING}

In comparison with other sensing materials, such as rare-earth ${ }^{192}$ or carbon nanotubes ${ }^{193}, 194$, GO was a low cost and transparent material with remarkable properties, such as facile functionalization of its large detection area, good electrical conductivity and biocompatibility ${ }^{195,196}$. Thus GO had been utilized in biological sensor devices in many different transduction modes ${ }^{197,198}$. GO had been applied as substrate in fluorescence quenching DNA detection schemes ${ }^{198-}$ 200. Balapanuru et al. $^{201}$ and Liu et al. ${ }^{202}$ studied fluorescence quenching properties of GO in DNA biosensing. GO sheets were also employed in arraystructured biosensor to recognize specific DNA hybridization ${ }^{203,204}$. GO sensor for the detection of multiple DNA targets in a single solution was developed by Tao et al. $^{205}$. Furthermore, fluorescence immunosensing based on GO microarrays had been used to detect rotavirus ${ }^{206}$. The quenching principle of graphene had also been utilized for aptamer-based detection of thrombin ${ }^{207}$.

Electrochemical detection of DNA had also attracted great attention, because DNA hybridization can be detected directly using oxidative signals of DNA bases or by using electroactive labels ${ }^{198,}{ }^{208}$. Since, chemically reduced GO provides high defect density and thus superior electrochemical performance ${ }^{198}$, all four DNA bases in ssDNA/dsDNA could be detected with high signal strength and higher sensitivity compared to graphite ${ }^{209}$. This feature was also 
beneficial in detecting single-base mismatches ${ }^{209}$. The electrochemical responses on reduced GO were studied comprehensively for various biomarkers including free-DNA bases, oxidase/ dehydrogenase-related molecules, neurotransmitters, ascorbic acid and acetaminophen ${ }^{210,211}$.

Besides GO-based optical ${ }^{212-215}$ and electrochemical biosensors ${ }^{209,}$ 216-218, biological sensors from GO field-effect transistors (FETs) had also attracted great interest. The architecture of GO FETs enables real-time, label-free yet sensitive measurements from readable electrical signals which arise between the interactions from target bio-molecule and the FET transducer surface ${ }^{197,219-}$ 221 . In conjunction with facile on-chip integration, high transconductance and the cost-effective fabrication, FET based biosensors can become an excellent alternative to existing biosensor technologies ${ }^{197,219}$.

In recent years, FETs based on thermally or chemically reduced graphene oxide had been successfully utilized to detect DNA hybridization by monitoring the conductance change of the FET device ${ }^{197,204,222}$. Stine et al. ${ }^{204}$ used reduced GO modified with DNA for detecting ssDNA with a detection limit of 10nM.

Agarwal et al. ${ }^{223}$ demonstrated the biocompatibility of reduced GO by functionalizing the FET device with proteins in order to detect various metal ions $^{224}$. He et al. ${ }^{11}$ fabricated a reduced graphene oxide biosensor for detecting the dynamic secretion of hormonal catecholamine from live cells.

Dong and co-workers reported the fabrication and operation of a highly sensitive and selective thermally reduced graphene oxide FET decorated with gold nanoparticle-antibody conjugates for detection of protein-binding events ${ }^{225}$. This approach of modifying the graphene surface with nanoparticles to increase the number of probe molecules at the FET gate was beneficial in terms of a linear and enhanced sensor response ${ }^{198}$.

The applications of graphene-based nanomaterials in biosensor platforms were manifold and had been proven by various studies. However, a large amount of work was needed to expand and adapt this biosensing technology to different areas. Medical applications for graphene-based immunosensors may include the detection of low quantities of cancer biomarkers. Such devices were beneficial 
in terms of an early detection of serious diseases, resulting in a contemporary successful treatment ${ }^{226}$. For the development of such a sensor system, multiplex detection of various cancer biomarkers comes into consideration, since it was far more likely that cancer can be detected at the early stage ${ }^{227}$. Further potential applications include the food production industry as well as the environment by detecting bacterial and viral pathogens in food or a diverse range of toxins ${ }^{226}$.

There had been developments on rGO transducer as molecular sensors, however, the sensitivity range was poor, labels and amplification was needed in addition to FET sensing (Table 5). The importance of both sodium and IL-6 analyte was discussed in earlier sections. Non-invasive, real-time detection of both Interleukin-6 and sodium ions can give important parameters for training and health monitoring purposes. 


\begin{tabular}{|c|c|c|c|c|}
\hline Ref & Transducer & $\begin{array}{c}\text { Detection range / } \\
\text { Sensitivity }\end{array}$ & Detection Capability & Pros/Cons \\
\hline 228 & $\begin{array}{c}\text { Thermally reduced } \\
\text { GO with gold } \\
\text { conjugate }\end{array}$ & $\begin{array}{c}2 \mathrm{ng} / \mathrm{mL}-0.02 \\
\mathrm{mg} / \mathrm{mL} \\
(13-137 \mathrm{nM})\end{array}$ & IgG Protein & $\begin{array}{l}\text {-Highly sensitive and } \\
\text { selective } \\
\text {-Suspended graphene } \\
\text {-Gold nanoparticle } \\
\text { amplification required }\end{array}$ \\
\hline 11 & $\begin{array}{c}\text { Chemically reduced } \\
\text { GO }\end{array}$ & $1 \mathrm{mM}$ & $\begin{array}{l}\text { Catecholamines from } \\
\text { attached PC12 cell } \\
\text {-dopamine, } \\
\text { epinephrine, } \\
\text { norepinephrine }\end{array}$ & $\begin{array}{l}\text {-Sensitive to cell } \\
\text { activities } \\
\text {-No selectivity }\end{array}$ \\
\hline 229 & $\begin{array}{c}\text { Chemically reduced } \\
\text { GO }\end{array}$ & $5 \sim 200 \mu \mathrm{M}$ & Dopamine & $\begin{array}{l}\text {-Selective detection of } \\
\text { dopamine } \\
\text {-Low sensitivity }\end{array}$ \\
\hline 222 & $\begin{array}{c}\text { Amine } \\
\text { functionalized } \mathrm{GO}\end{array}$ & $\begin{array}{c}\text { Single bacteria and } \\
\text { DNA detection }\end{array}$ & Bacteria and DNA & $\begin{array}{c}\text {-Novel way of } \\
\text { functionalizing } \\
\text { graphene } \\
\text {-High sensitivity } \\
\text {-No selectivity on } \\
\text { detection of bacterium } \\
\text { and DNA }\end{array}$ \\
\hline
\end{tabular}

From the comparison of currently available and practical sodium sensors (Table 6), although the sensors were able to detect sodium in the physiological range of ca. $10-100 \mathrm{mM}$, there was a lack of a flexible, disposable, low-cost sodium sensor suitable for real-time sensing in field conditions. 
Table 6: A comparison of currently available and practical sodium sensors

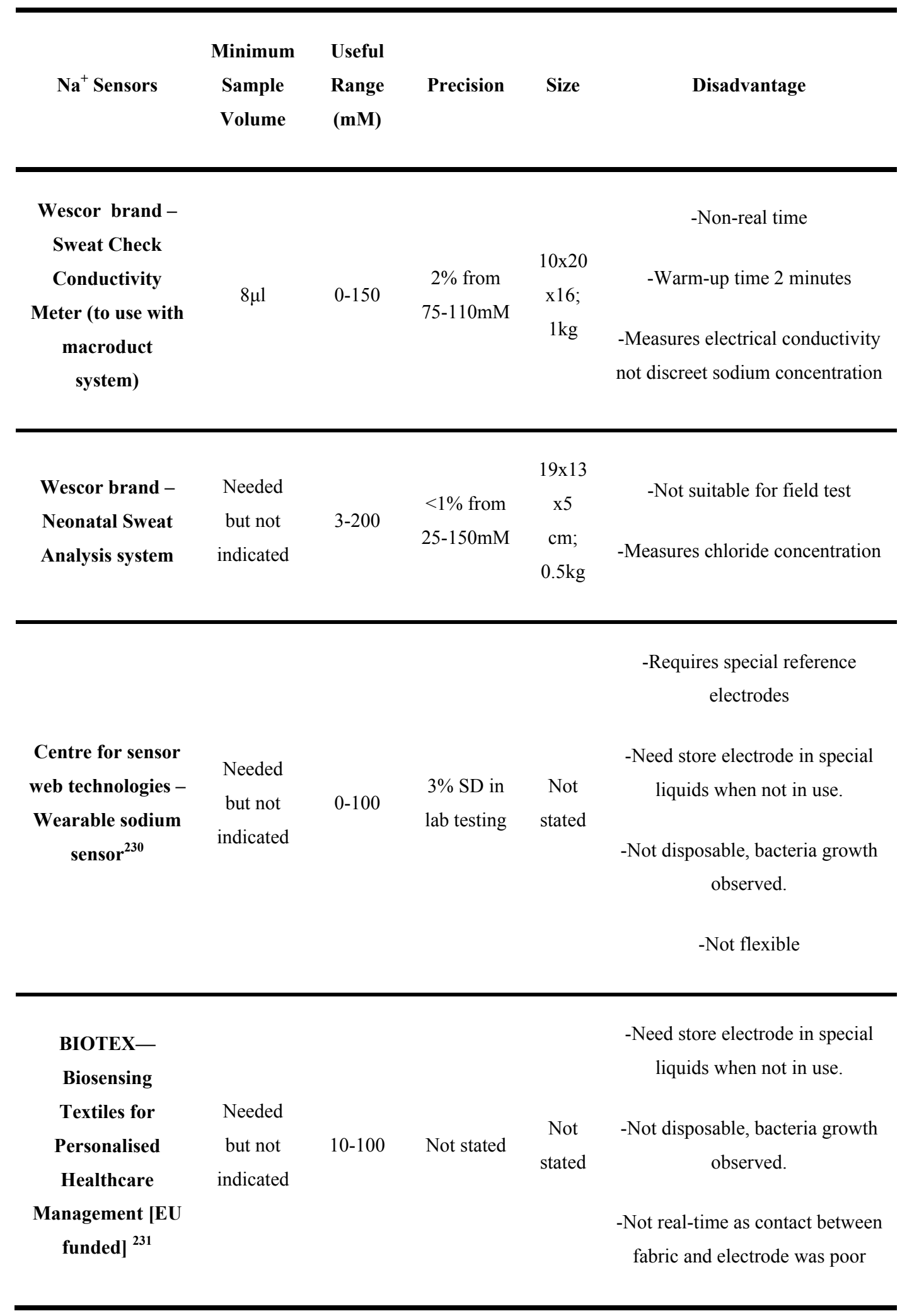


In conclusion, extraordinary electrical, chemical and optical properties along with its facile, low-cost and aqueous processable route, suggested that GO could be introduced in a wide variety of biosensors. New synthesis methods such as improved chemical oxidation and the synthesis of large GO flakes showed good potential for the next-generation of biosensors. GO had shown promising sensitivity and selectivity in field-effect transistor biosensors. The future of GO was highly promising although more research work was needed to fully explore and control the benefits of this remarkable material and convert these works into practical biosensors, in this thesis demonstrated for sodium ions and IL-6 proteins. 


\section{CHAPTER 2 INTRODUCTION}

\subsection{CURRENT Challenges AND Motivations}

Reduced graphene oxide (rGO) had huge advantages over other methods of graphene synthesis due to it being solution processable, industrially scalable and having a tuneable electronic band-gap as highlighted in the previous chapter. However, there were some limitations that prevent rGO to be a practical and ultra-sensitive transducer in molecular sensing applications. Firstly, rGO had low trans-conductance due to high intrinsic electrical resistance. Secondly, rGO cannot be fabricated into electronics with good electrical homogeneity. These two limitations need to be resolved before rGO can be used as sensor transducers.

The continuous monitoring of individual sodium loss in sweat was important because every individual had different sodium loss rate during physical activities and an appropriate fluid replacement regimen must be adapted to the individual to prevent the onset of hyponatraemia. However, there was no realtime monitoring system for sodium in sweat available commercially.

Interleukin-6 (IL-6) was an important multi-functional cytokine with a wide range of biological activities. It could provide information and predict events such as the onset of overtraining and glycogen depletion and high chronic inflammation which underpinned many chronic diseases. Current detection of circulating IL-6 could only be measured invasively with subsequent laboratory analysis and thus could not provide critical, immediate and continuous feedback.

There was a niche to develop a facile and ultra-sensitive transducer for sodium and IL-6 monitoring that was non-invasive, reproducible, real-time and lowcost. The biosensors market, which was currently at USD 9.9 billion, was expected to reach USD 18.9 billion in 2019. Thus it was commercially viable to conduct research in this area. 


\section{$\underline{2.2 \text { AIM }}$}

The aim for this $\mathrm{PhD}$ thesis was to eliminate the electrical limitations in $\mathrm{rGO}$, through novel methods, while revealing more of the condensed matter properties of GO. Thereafter, develop a biosensor platform for detection of sodium and Interleukin-6 analyte. The thesis aimed to test the hypotheses and elucidate the mechanisms involved.

\subsection{OBJECTIVE}

The main objective of this thesis was to develop an ultra-sensitive carbon-based sensor that can measure both the sodium ions in the millimolar range and the IL-6 proteins in the picogram range. In order to do so, five sub-objectives need to be achieved. (1) Conduct a critical literature review of current rGO synthesis techniques, sodium biosensors and IL-6 biosensors. (2) Obtain ultra-large GO sheets to improve trans-conductance of rGO. (3) Produce rGO-based electronics with good electrical conductivity and homogeneity. (4) Fabricate a field-effect transistor biosensor platform. (5) Obtain physiological relevant rGO-based biosensor for both sodium and IL-6.

\subsection{HYPOTHESES}

This thesis set out to test the hypothesis that the rGO low electrical conductivity, high electrical deviation and low manufacturability could be eliminated to create a highly sensitive biosensor transducer via (A) improving rGO synthesis (B) pristine Carbon Nanotubes-rGO hybrid film and (C) growth of rGO. This hypothesis can be sub-divided into four hypotheses for testing.

Firstly, it was understood that synthesized GO from graphite consists of varying sizes and further post-processing was needed to separate the useful large flakes. A hypothesis was that size fractionation to get ultra-large GO sheets during the graphite oxidation was possible. It was further speculated that it was due to a 
combination of the size dependent amphiphilicity of GO (large GO float) and strong van der Waal's attraction between large GO/Graphite.

Secondly, GO-CNT hybrid films possess a wide spectrum of useful properties including enhanced electron-transport path and high optical transparency. It was understood that large GO-CNT hybrid films can only be obtained using chemical modification for fabrication. However, it was hypothesized that by removing facile oxygen moieties on GO, pristine GO-CNT hybrid film can be obtained due to decreased electrostatic repulsion and the relative hydrophobicity between the constituents.

Thirdly, GO were flake-like and their arrangement on surfaces were inhomogeneous. It was understood that Graphene can be grown using CVD treatment with metal catalyst. A hypothesis was that rGO can be grown from pre-existing GO templates without any metal catalysts. It was further speculated that the growth supports the free radical condensate theory and that the fabricated rGO device had improved electrical homogeneity.

Lastly, the rGO synthesized can be used for the (1) physiological relevant detection of sodium in sweat via a disposable all-organic biosensor and (2) ultra-sensitive detection of IL-6 in circulation via a field-effect-transistor based biosensor.

\section{$\underline{2.5 \mathrm{SCOPE}}$}

In these studies, the chemical exfoliation method to obtain graphene was used due to its advantage of low-cost, aqueous and industrial-scalable production. The thesis aimed to significantly decrease rGO electrical resistivity yet increase its homogeneity to obtain a sensitive biosensor transducer. The report also focused on detection of only sodium ion and IL-6 protein analyte because these were importance biomarkers and there was no real-time sensor available. 


\section{$\underline{2.5 \text { OUTLINE OF THESIS }}$}

This $\mathrm{PhD}$ thesis report was divided into 3 main parts. Firstly, a critical literature review on GO structure, GO synthesis, sodium in sweat, IL-6 in circulation and GO biosensors; and the introduction of this $\mathrm{PhD}$ thesis were covered in Chapter 1 and 2. Secondly, results and discussions on the improved synthesis of GO, sodium biosensor, synthesis of CNT-GO hybrid material, growth of rGO and IL-6 biosensors were covered under Chapters 3-7. Thirdly and finally, the conclusion and future work recommendations were presented in Chapter 8 . 


\section{Chapter 3: Preparation AND \\ MECHANISM OF ULTRA-LARGE \\ GRAPHENE OXIDE FLAKES}

In this chapter, an improved Hummer's method that yielded the largest average graphene flake size reported to date was achieved. The large graphene oxide sheets had an average size of $\sim 700 \mu \mathrm{m}^{2}$ which was 7 times larger and had much higher average hole mobility of $\sim 159 \mathrm{~cm}^{2} / \mathrm{Vs}$ than previously reported literatures. Through a systematic manner, the role and mechanism of preoxidation and in-situ size fractionation in modified Hummer's method was elucidated. This study allowed large mono-layered graphene oxide to be obtained, and was subsequently used for other experiments in the following chapters.

\subsection{INTRODUCTION}

Graphene, a single-atom-thick and two-dimensional carbon nanomaterial ${ }^{58}$, had attracted increasing attention in various applications including nanoelectronics $^{232}$, energy storage $^{233}$, field-effect transistors (FETs) ${ }^{234,} 235$ and sensors ${ }^{4,11,41,54,59,236}$ because of its unique physical and electrical properties ${ }^{237-}$ 239. So far, single or few-layered graphene had been prepared by various methods including mechanical exfoliation ("Scotch-tape" method) 4, 54, 55, chemical vapour deposition $(\mathrm{CVD})^{58,59}$ and chemical reduction of graphene oxide $(\mathrm{GO})^{11,41}$. Whereas the low productivity of the mechanical cleavage made it unsuitable for large-scale use, the synthesis of graphene by high temperature chemical vapour deposition (CVD) technique was complicated, expensive and often time-consuming. Moreover, CVD technique was not compatible with plastic or cellulose substrates ${ }^{11}$. Alternatively, graphene oxide was highly suitable for low cost and large scale production of graphene. Indeed, previously reported methods resulted in the production of large graphene oxide 
(GO) flakes ${ }^{13,240}$ but often with only moderate electrical conductivity. It was frequently reported that efficient reduction of GO thin films was only achieved through a combination of hydrazine vapour exposure and temperature annealing treatment ${ }^{13,85}$. Although thermal reduction of graphene oxide (GO) sheets resulted in improved electrical properties of the devices ${ }^{12}$, this method could not be used thoroughly, particularly with regard to thermally unstable substrates such as plastic materials. Hence, besides a facile and reproducible method appropriate for the large-scale productions of single or few-layer $\mathrm{GO}$, the reduction process of GO sheets could be improved leading to rapid and largescale fabrication of graphene-based devices suitable to apply in the area of electronics and molecular sensors.

This chapter reported a modification of Hummers' method, resulting in readily exfoliated graphene oxide (GO) sheets, which could be reduced by hydrazine vapour more effectively. With this approach, an increase in the total yield of GO sheets was also noted. When GO sheets, up to $3500 \mu \mathrm{m}^{2}$ in size, were deposited on appropriate $\mathrm{SiO}_{2} / \mathrm{Si}$ wafer, the subsequent reduction process using hydrazine vapour resulting in remarkable lower sheet resistance and higher hole mobility of the devices than resulted from the original method ${ }^{13}$. No additional annealing step after hydrazine reduction was needed to achieve high conductivities of the graphene flakes. Electronic transfer measurements on the graphene devices indicate that they were very stable over a broad range of $\mathrm{V}_{\mathrm{g}}$. Due to the fact that the fabricated graphene FET operates at low-voltage making it suitable for molecular sensing applications. With larger flakes, the success rate of conductive FET devices fabrication increased from $\sim 20 \%$ ( $40 \mu \mathrm{m}$ graphite) to $\sim 100 \%$.

The graphene oxide (GO) synthesis method described by Su et al. $2009^{13}$ was modified by taking only large floating flakes after both the ultra-sonication and oxidation steps for further processing. According to ref. ${ }^{13}$, a graphite preoxidation procedure was needed to avoid incompletely oxidized graphitecore/GO-shell particles in the final product. Due to the large size of the natural graphite flakes $(2-5 \mathrm{~mm})$, it was expected that the floating flakes were not fully oxidized, so GO sheets were weakly attached to the graphite core. 
In the synthesis, large floating flakes were collected after the main oxidation step and re-suspended into $500 \mathrm{ml}$ DI water by stirring overnight. Due to the mechanical movement, large GO flakes that were weakly adhered to the core peeled off. Since GO was soluble in water because of its carboxyl-and hydroxyl groups, exfoliation was achieved by this dilution step. In contrast to similar considerations $^{13,85}$, no ultra-sonication was used. By this facile but crucial resuspension step addition, the total yield of GO sheets was increased to $25 \%$.

\subsection{IMPROVED PROCEDURE FOR LARGE GO FLAKES}

\subsubsection{MATERIALS}

Graphite flakes (3-5mm) were obtained from NGS Naturgraphit $\mathrm{GmbH}$, Leinburg, Germany. $\mathrm{H}_{2} \mathrm{SO}_{4}, \mathrm{KMnO}_{4}$, (3-Aminopropyl)Triethoxysilane (APTES) and hydrazine monohydrate were obtained from Sigma Aldrich. $\mathrm{H}_{2} \mathrm{O}_{2}$ was obtained from Pro Analysi, Merck. All chemicals obtained were in research grade and used without further purification. De-ionized water was used throughout the experiment.

\subsubsection{SYNTHESIS OF LARGE GO}

Graphene oxide (GO) sheets were prepared by the modified Hummers' method from natural graphite flakes (average size was $3-5 \mathrm{~mm}$ ). Briefly, $2 \mathrm{~g}$ graphite flakes were mixed with concentrated $\mathrm{H}_{2} \mathrm{SO}_{4}(12 \mathrm{ml})$ and stirred for 4.5 hours at $80{ }^{\circ} \mathrm{C}$. After cooling to room temperature, the flakes were then ultra-sonicated (300w) for 1 hour and diluted with DI water $(500 \mathrm{ml})$ and left overnight. The mixture was filtered through porous filter to obtained pre-oxidized graphite powders. The product was dried overnight in vacuum desiccator.

The dried graphite powder was then added slowly into concentrated $\mathrm{H}_{2} \mathrm{SO}_{4}$ $(120 \mathrm{ml})$ and $\mathrm{KMnO}_{4}(15 \mathrm{~g})$. The solution was stirred for 2 hours at room temperature and then diluted with DI water $(250 \mathrm{ml})$ in an ice-bath. After another 2 hours stirring, DI water $(700 \mathrm{ml})$ was added into the solution, and 
shortly thereafter, $\mathrm{H}_{2} \mathrm{O}_{2}(20 \mathrm{ml})$ was added into the mixture, resulting in a colour change to light yellow. Only the upper portion of the mixture (floating flakes) was collected and filtered. Finally, the resulting solid was stirred overnight in DI water $(500 \mathrm{ml})$ to obtain a stock solution of GO (unreacted graphite powders settled to the bottom of the solution). This solution was then centrifuged at $5000 x g$ for 3 minutes and further purified by dialysis (cut-off $3000 \mathrm{MW}$ ) against DI water for one week.

\subsubsection{REDUCTION OF GO}

Single-and few-layer GO sheets were deposited onto silicon substrates with $300 \mathrm{~nm}$ silicon oxide layer by drop-cast method. Silicon substrates were first cleaned with a standard RCA solution to remove undesired impurities. Subsequently, the cleaned $\mathrm{SiO}_{2}$ substrates were modified with APTES selfassembled monolayers (SAMs), in order to enhance the adsorption of graphene oxide. Therefore, the substrates were immersed into a 1\% APTES solution in ethanol for $1 \mathrm{~h}$, followed by extensively rinsing with absolute ethanol, before baking for 2 hours at $120^{\circ} \mathrm{C}$. GO solution $(1.3 \mathrm{mg} / \mathrm{ml})$ was drop-casted onto the devices for 1 hour, followed by thoroughly rinsing with DI water and drying with $\mathrm{N}_{2}$. For the hydrazine vapour reduction, samples were put into glass petri dishes and $500 \mu 1$ of $98 \%$ hydrazine monohydrate was placed in the centre of the petri dish. The samples were kept in the sealed petri dish at $70{ }^{\circ} \mathrm{C}$ for $16 \mathrm{~h}$.

\subsection{MECHANISM OF LARGE GO FLAKES}

It was noted that although pre-oxidation steps had been commonly used in synthesis of aqueous graphene, the role and mechanism of pre-oxidation on graphite had yet to be determined. For smaller graphite flakes, the first step of pre-oxidation was used to obtain a higher yield of GO from micron-sized graphite $^{91}$. Then in $2009, \mathrm{Su}$ et $\mathrm{al}^{13}$ used ultra-sonication on large graphite flakes $(3-5 \mathrm{~mm}$ across) to substitute chemical pre-oxidation step and this resulted in large flakes with higher mobility values up to $12 \mathrm{~cm}^{2} / \mathrm{Vs}$. Adding a 
pre-oxidation step to the Hummer's method, was reported to enlarge the size of aqueous graphene ${ }^{13,86}$. With larger flakes, the carrier mobility and the electrical conductivity ${ }^{87}$ could be increased while maintaining high optical transparencies ${ }^{39}$.

Ultra-sonication was used as a pre-oxidation step in the reported synthesis as well (Figure 19). After this step, small graphite debris was broken off from the graphite cores. The weight and average size (Dynamic Light Scattering) of the debris removed were $0.57 \mathrm{~g}$ and $6.79 \mu \mathrm{m}$ respectively. As the maximum size of aqueous graphene obtained was limited to the largest size of graphene domains in the graphite flake from which it was chemically synthesized ${ }^{43}$, the broken down debris were removed.

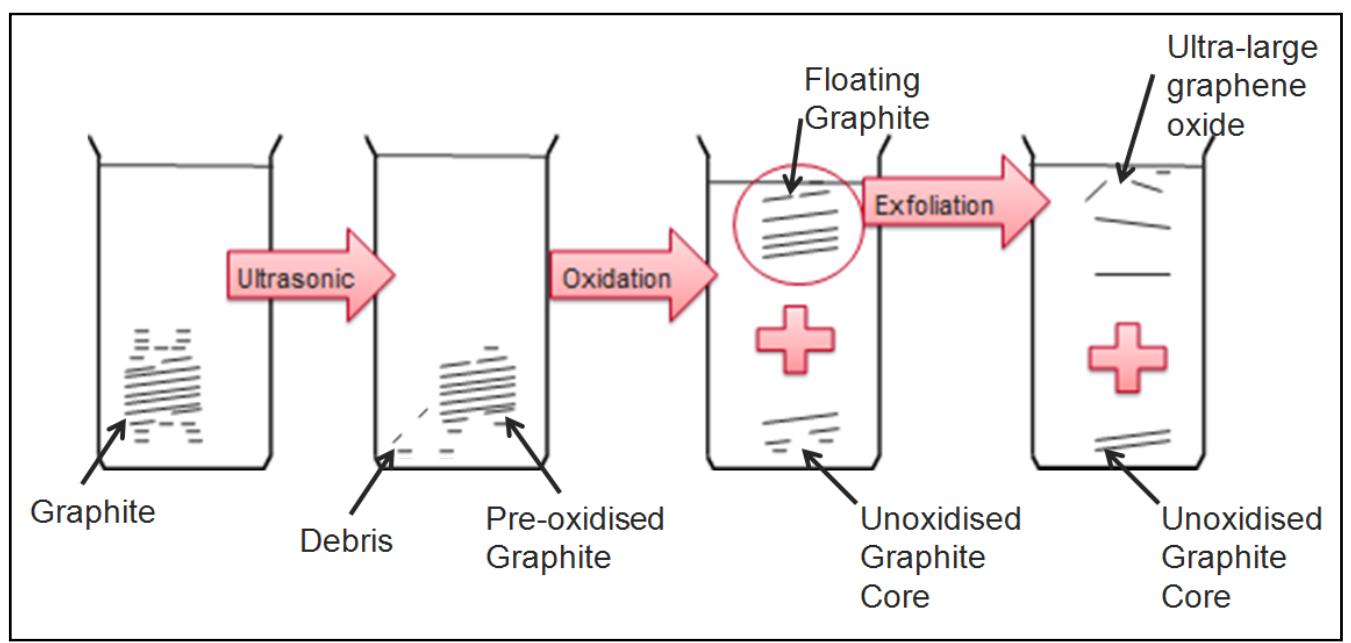

Figure 19: Schematic of improved GO synthesis

The pre-oxidised graphite was then dried in vacuum desiccator before being oxidised with the strong oxidising agents. Literatures ${ }^{13,241,242}$ had reportedly used mild heat to dry the pre-oxidised GO at this stage. It was detrimental to the $\mathrm{GO}$ and it reduces the GO permanently. Even usage of mild heating at $70^{\circ} \mathrm{C}$ reduced the GO. This reduction could be measured using the water contact angle measurement shown in Figure 20. Drying under mild heating $\left(70{ }^{\circ} \mathrm{C}\right)$ increased the contact angle by $\sim 10^{\circ}$ from $51.8^{\circ}$, whereas vacuum desiccator 
sample only increased by $1.8^{\circ}$. Although the GO after drying in vacuum desiccator was not fully removed of water ${ }^{243}$, but it was safe for reaction with the concentrated acid in subsequent step.

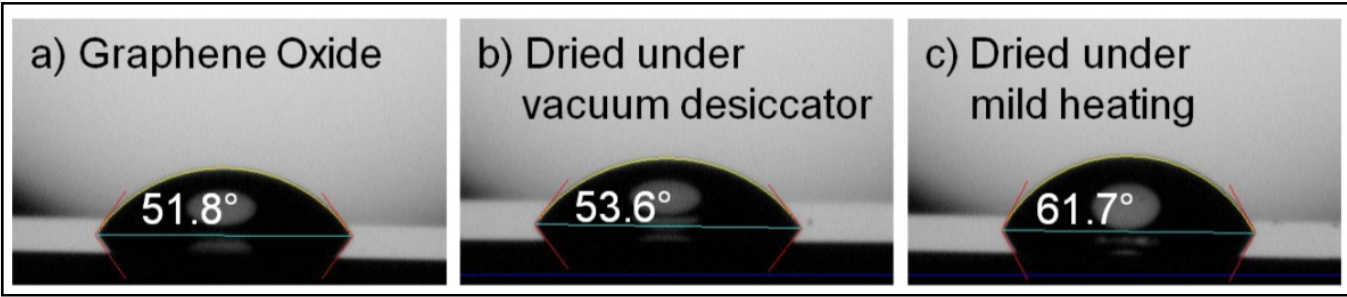

Figure 20: Water Contact Angle measurement for (a) Graphene oxide, (b) GO dried under vacuum desiccator and (c) GO dried under mild heating.

Table 7: Comparison of GO yield concentration between oven-dried and vacuum-dried pre-oxidised samples (the GO solution was obtained after centrifuging at $5000 \mathrm{G}$ for 3mins).

\begin{tabular}{|c|c|c|c|c|c|}
\hline $\begin{array}{c}\text { Freeze Dried } \\
\text { Samples }\end{array}$ & Samples & $\begin{array}{c}\text { Empty Tubes } \\
\text { (mg) }\end{array}$ & $\begin{array}{l}\text { Final } \\
\text { Weight } \\
\text { (mg) }\end{array}$ & $\begin{array}{l}\text { Conc. } \\
(\mathrm{mg} / \mathrm{ml})\end{array}$ & $\begin{array}{l}\text { Average } \\
\text { Conc. } \\
(\mathrm{mg} / \mathrm{ml})\end{array}$ \\
\hline \multirow{2}{*}{ Oven-dried } & A & 993.23 & 993.48 & 0.25 & \multirow{2}{*}{0.1950} \\
\hline & B & 1005.17 & 1005.31 & 0.14 & \\
\hline \multirow{3}{*}{ Vacuum-dried } & A & 985.91 & 986.45 & 0.54 & \multirow{3}{*}{0.5650} \\
\hline & B & 996.22 & 996.81 & 0.59 & \\
\hline & B & 1037.82 & 1039.1 & 1.28 & \\
\hline
\end{tabular}

From Table 7, the final GO yield concentration difference between oven and vacuum dried sample was significant. The concentration obtained from the 
oven-dried samples was 2.9 times less than the vacuum-dried. Thus, from the contact angle measurement and the GO concentration measurements, ovendrying was detrimental to the yield of GO synthesis.

The dried pre-oxidised graphite was further oxidised. A mixture of floating graphite flakes and sunken graphite flakes was obtained after synthesis (Figure 21). It was hypothesized that some graphite float because larger GO were adhere to the graphite core due to size dependent amphiphilicity of GO.

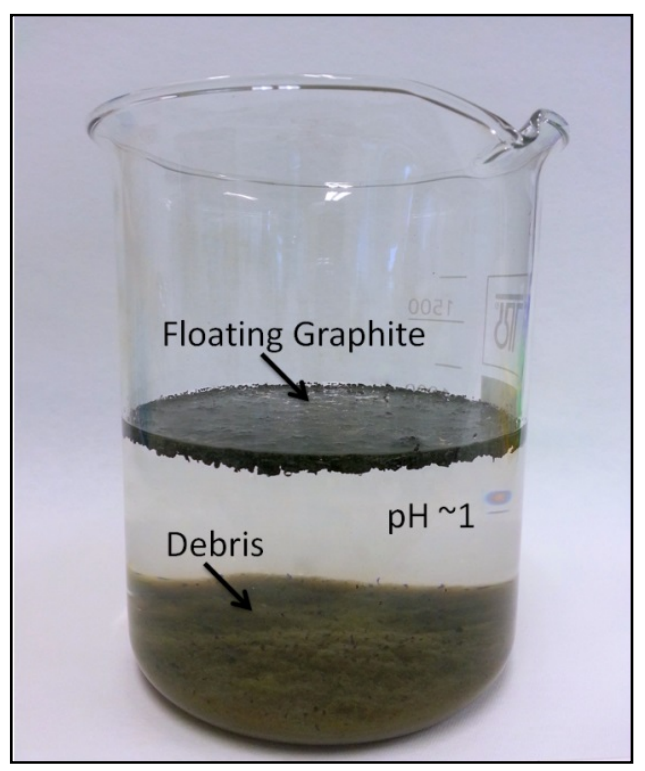

Figure 21: After main oxidation step, two distinct layers were obtained - floating graphite layer and the debris layer

Subsequently, only large floating flakes were used for further oxidation. After the pre-oxidation, no further ultra-sonication was used to re-disperse or exfoliate the aqueous flakes which could result in smaller sheets, in contrast to using ultra-sonication method for exfoliation ${ }^{244,245}$.

The floating flakes were removed from the original mixture and transferred to a new container with fresh DI water for subsequent exfoliation. The exfoliation of the aqueous graphene from the graphite core was instead carried out by mild stirring in DI water. The mechanical movement helped the large aqueous 
graphene flakes that were all weakly adhered to the core peel off. The higher $\mathrm{pH}$ value also helped with the effective exfoliation.

Figure 22a showed the mixture after this mechanical exfoliation. After centrifugation, three distinct portions was obtained - a lower graphite portion with un-oxidised graphite(Figure 22b), middle portion with some un-oxidised graphite (Figure 22c) and upper portion with completely exfoliated GO flakes (Figure 22d).

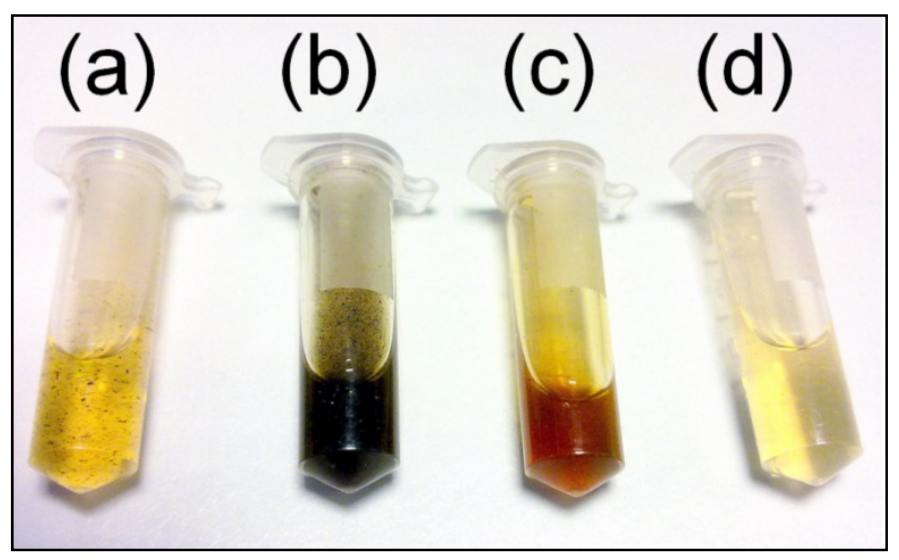

Figure 22: (a) Mixture after mechanical stirring of the large floating GO flakes, before centrifugation. After centrifugation, the solution showed 3 distinct portions (b) lower graphite portion with un-oxidised graphite, (c) middle portion with some un-oxidised graphite and (d) upper portion with GO flakes

Different portions obtained from the GO mixture were used for fabrication of $\mathrm{GO}$ on $\mathrm{SiO}_{2}$ chips. From Figure 23, the fabricated chip from the top portion of the GO synthesis mixture after centrifugation was generally well-covered with large GO flakes with exception of some gaps. The $\mathrm{SiO}_{2}$ fabricated from the middle portion showed multi-layered GO sheets which were not completely exfoliated, while the lower portion showed unreacted graphite. Both the middle and lower portion of the GO synthesis mixture were thus not suitable for applications that required single layer of GO. They were however, suitable for electrodes and electronics devices which did not require single layer GO. 


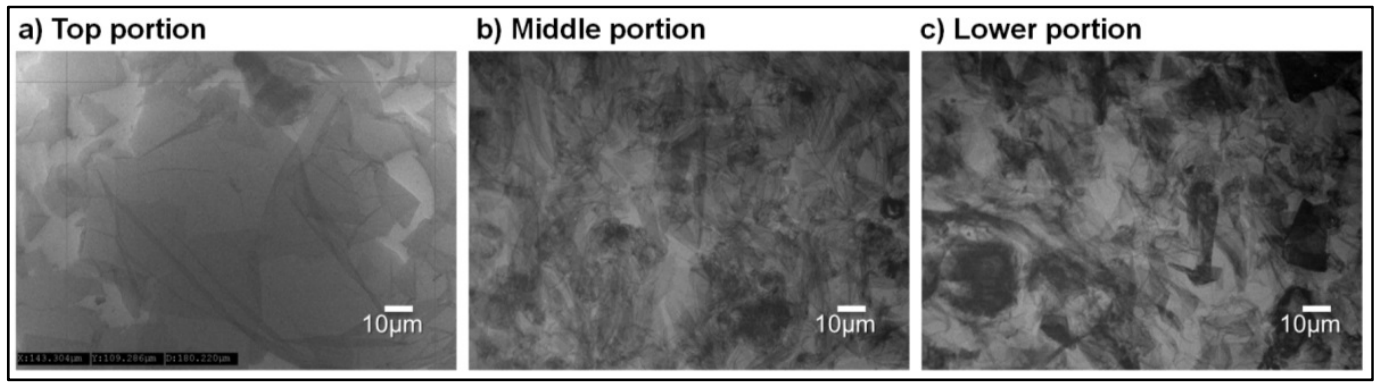

Figure 23: SEM images of GO flakes on $\mathrm{SiO}_{2}$ from the (a) top (b) middle and (c) lower portion part of the GO synthesis mixture after centrifugation.

Dynamic mechanical analyser (DMA) was used to obtain the force needed to fully delaminate the top layer of GO and graphene from the oxidised graphite core and graphite core respectively. The average force needed for the graphite and GO was $0.330 \mathrm{~N}$ and $0.012 \mathrm{~N}$ respectively. The force needed to delaminate GO was $\sim 30$ times smaller than that of graphite. Thus a gentle exfoliation step after the oxidation by the mechanical stirrer was sufficient to separate the GO from the oxidised graphite core.

Table 8: Exfoliated GO concentration obtained after the samples were oven-dried, vacuum-dried and membrane-dialysed

\begin{tabular}{|c|c|c|c|c|c|}
\hline $\begin{array}{c}\text { Freeze Dried } \\
\text { Samples }\end{array}$ & Samples & $\begin{array}{l}\text { Empty } \\
\text { Tubes } \\
(\mathrm{mg})\end{array}$ & $\begin{array}{l}\text { Final } \\
\text { Weight } \\
\text { (mg) }\end{array}$ & $\begin{array}{l}\text { Conc. } \\
(\mathrm{mg} / \mathrm{ml})\end{array}$ & $\begin{array}{l}\text { Average } \\
\text { Conc. } \\
(\mathrm{mg} / \mathrm{ml})\end{array}$ \\
\hline \multirow{2}{*}{$\begin{array}{c}\text { Vacuum-dried, } \\
\text { exfoliated at } \\
\sim \text { pH } 1\end{array}$} & A & 985.91 & 986.45 & 0.54 & \multirow{2}{*}{0.5650} \\
\hline & B & 996.22 & 996.81 & 0.59 & \\
\hline \multirow{2}{*}{$\begin{array}{c}\text { Vacuum-dried, } \\
\text { exfoliated at } \\
\sim \text { pH } 4\end{array}$} & A & 1037.41 & 1038.72 & 1.31 & \multirow{2}{*}{1.2950} \\
\hline & B & 1037.82 & 1039.1 & 1.28 & \\
\hline
\end{tabular}


The physical transferring of the GO flakes into another container of DI water for exfoliation increased the effectiveness of GO exfoliation. The exfoliation of GO was improved at $\mathrm{pH} 7$ compared to more acidic $\mathrm{pH}$ due to the presence of $\mathrm{COOH}$ groups decreased the net energetic cost or enthalpy of mixing ${ }^{246}$ and increasing the interaction between solvent and the GO basal planes. To confirm if neutral $\mathrm{pH}$ was better for exfoliation, the un-exfoliated floating flake was membrane-dialysed using 3,000 MWCO membranes before centrifugation. Then the membrane was sealed and placed in a large reservoir of DI water under stirring. The result was tabulated in Table 8 where the average concentration of membrane-dialysed GO was increased 2.3 times, from 0.5650 to $1.2950 \mathrm{mg} / \mathrm{ml}$. Thus showing that neutral $\mathrm{pH}$ was advantageous to exfoliation.

Thus it was shown that large flakes using the reported improved synthesis method were obtained due to (1) larger graphite precursor, (2) physical size fractionation and (3) neutral $\mathrm{pH}$ exfoliation.

\subsection{CHARACTERIZATION OF LARGE GO FLAKES}

\subsubsection{SEM AND AFM CHARACTERIZATION}

The scanning electron microscopy (SEM) image of an ultra-large hydrazine vapour reduced GO flake, which was approximately $60 \mu \mathrm{m}$ in lateral size deposited on $\mathrm{SiO}_{2} / \mathrm{Si}$ substrate, was shown in Figure 24a. Smaller GO fragments were also observed beside the ultra-large flakes. Figure 24b illustrates a size histogram of GO flakes observed using optical microscopy and SEM. The average size of the GO flakes obtained by the reported method was $700 \mu \mathrm{m}^{2}$, hence the GO flakes were $\sim 7$ times larger in area than those produced using previously reported method ${ }^{240}$. 

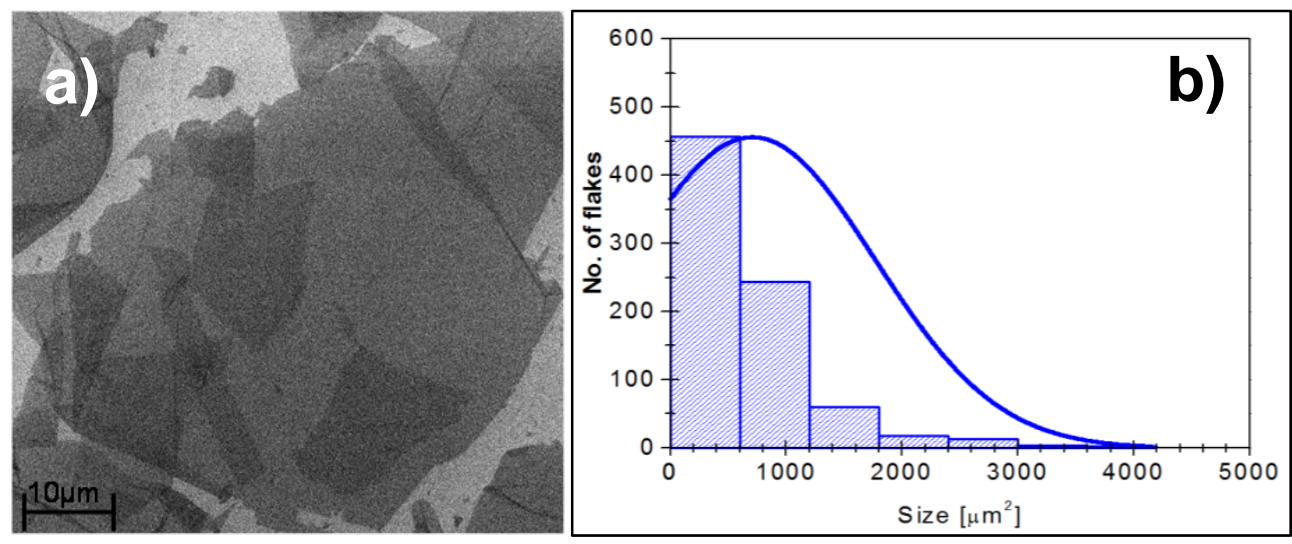

Figure 24: (a) SEM image of a large GO sheet on $\mathrm{SiO}_{2}$ substrate produced from the modified method. (b) Size distribution of GO flakes.

Figure 25 showed a typical tapping mode AFM image of GO deposited on a silica substrate by drop-casting method. The thicknesses of monolayer GO flakes were typically in the range of $0.7-1 \mathrm{~nm}^{40,85,247,248}$. From the AFM height profile it was shown that the thickness for the GO sheet obtained by the reported modified method was $\sim 1 \mathrm{~nm}$, suggesting that mono-layered GO could be proven over a large area.

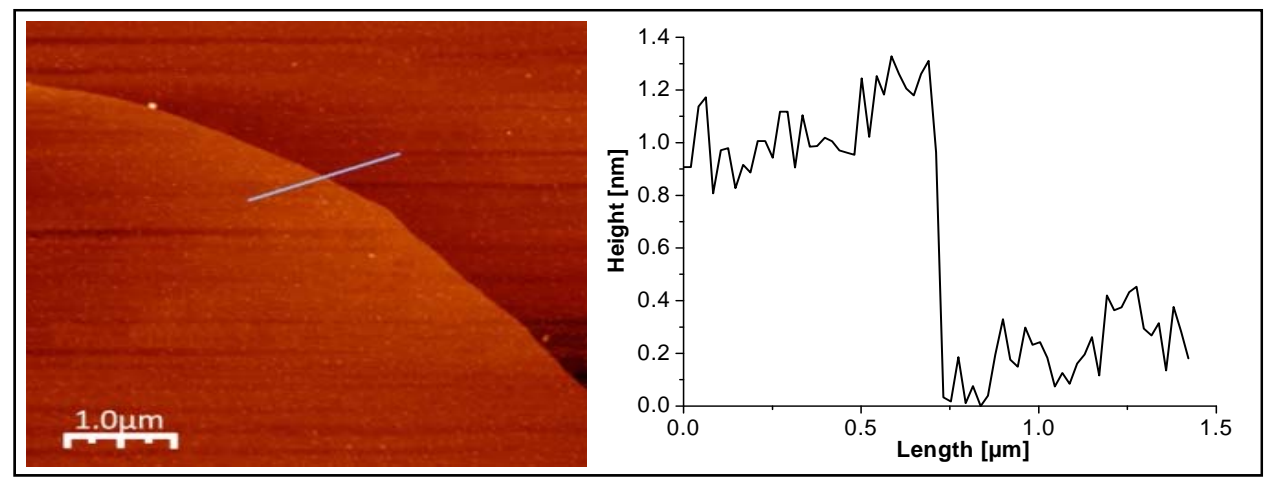

Figure 25: (Left) AFM image of GO and (Right) AFM height profile for the GO sheet 


\subsubsection{RAMAN AND XPS CHARACTERIZATION}

As shown in Figure 26 the Raman spectrum of GO displayed two prominent peaks at $1341 \mathrm{~cm}^{-1}$ and $1601 \mathrm{~cm}^{-1}$, which corresponded to the well-documented $\mathrm{D}$ and $\mathrm{G}$ bands. The Raman spectrum of $\mathrm{rGO}$ also exhibits both $\mathrm{D}$ and $\mathrm{G}$ bands (at 1330 and $1595 \mathrm{~cm}^{-1}$, respectively). The $\mathrm{D} / \mathrm{G}$ intensity ratio was increased in comparison with that of the GO spectrum. The Raman spectral results described above were in good agreement with those reported by Stankovich ${ }^{249}$, indicating that reduction took place in the GO.

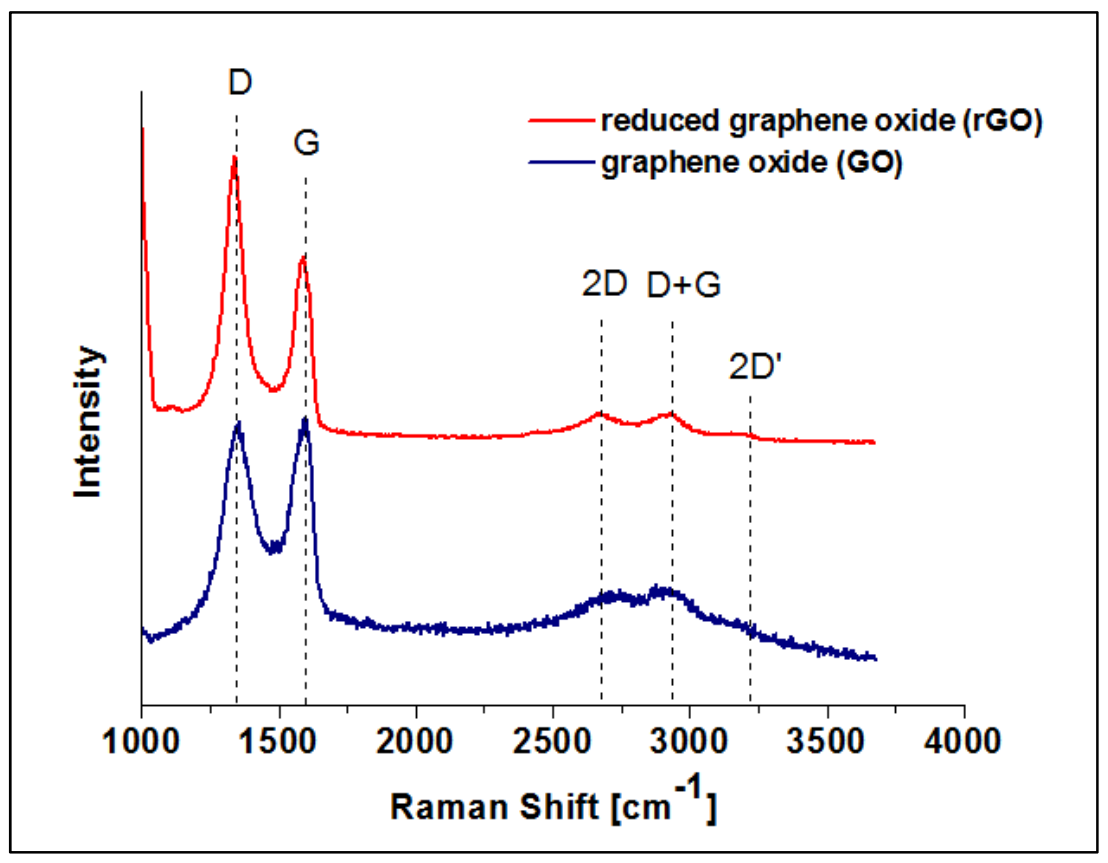

Figure 26: Raman spectra for GO and rGO

X-ray photoemission spectroscopic (XPS) measurements were performed for the as-prepared GO sheets and those after reduction with hydrazine vapour. The C1s spectrum of GO (Figure 27) indicated the presence of four types of carbon bonds: C-C (284.6 eV), C-O (286.7 eV), C=O (287.5 eV), O-C=O (288.8 eV). After hydrazine reduction, the peak intensities of oxide species were much smaller than those in the spectrum of GO, suggesting considerable deoxygenation by the reduction process ${ }^{91}$. Furthermore, an additional component at $285.6 \mathrm{eV}$ appeared, corresponding to the binding energy of $\mathrm{C}=\mathrm{N}$, consistent with previous reports $245,249,250$. 


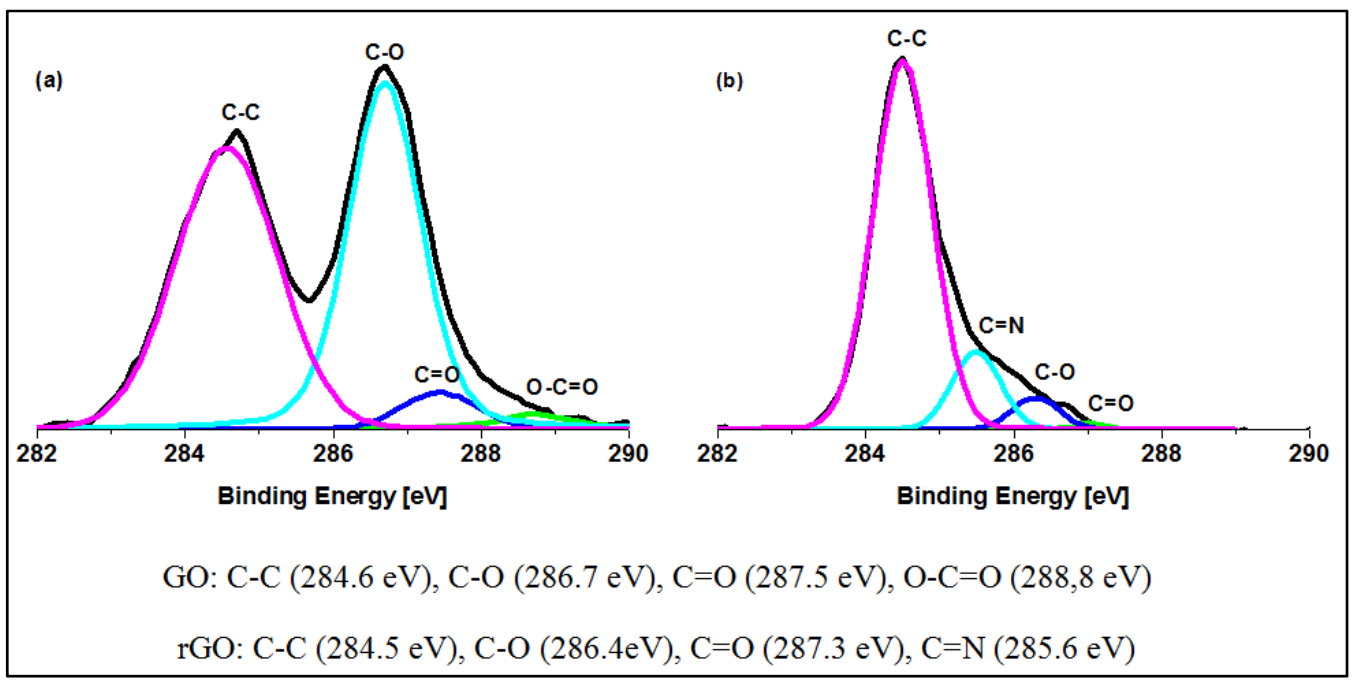

Figure 27: C1s XPS spectra for a) GO, b) reduced GO. Peaks corresponding to different carbon bonds were indicated

\subsubsection{FET Fabrication and Electronic Properties}

The hydrazine reduced GO sheets were tested regarding to their abilities to serve as conducting thin-film electrodes. Bottom-gate operated transistors were fabricated, without using elaborate electron-beam lithographic techniques which could leave polymer residue, by evaporating Au electrodes (100nm thick) directly on top of the selected rGO films that were previously deposited on $300 \mathrm{~nm} \mathrm{SiO}_{2} / \mathrm{Si}$ substrates.

Shadow mask were specially designed and fabricated on stainless steel template with extended winds and enlarged contact pads for robust and easy connection to external measurement equipment. Autodesk Autocad was used for the design shown in full as Figure 28a, and the magnified design as Figure $28 \mathrm{~b}$. 


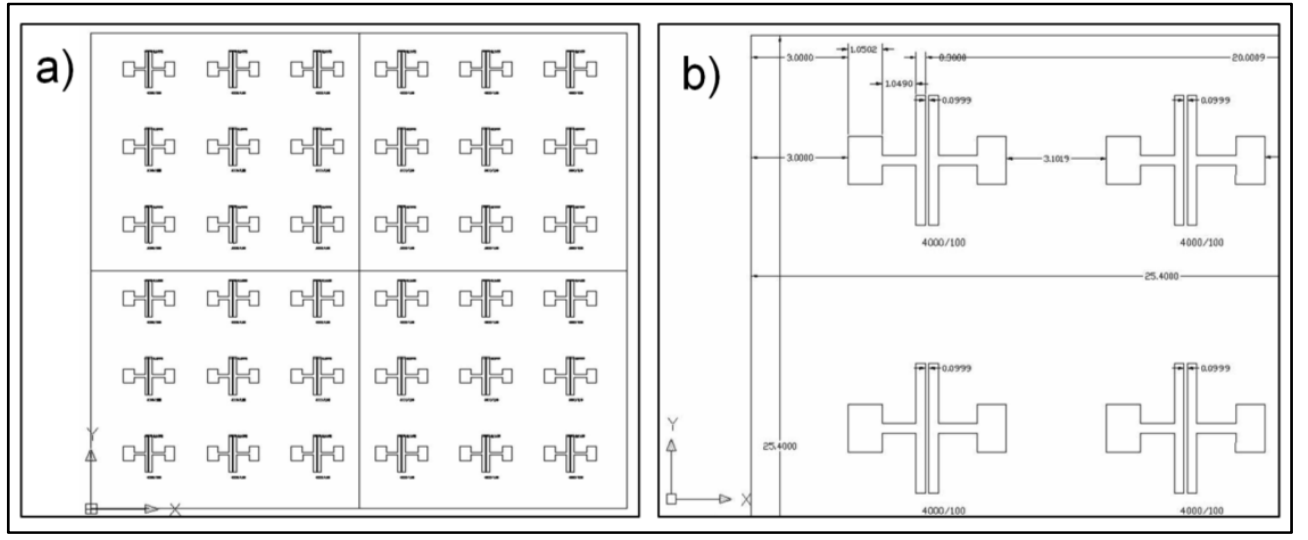

Figure 28: (a) Full and (b) magnified, image of Shadow mask design for FET fabrication

Figure 29a showed an optical micrograph of a typical fabricated device. It was frequently reported that hydrazine reduction was not sufficient to achieve high conductivities of the flakes, so further reduction by annealing in $\mathrm{Ar} / \mathrm{H}_{2}$ at high temperatures was necessary ${ }^{13,85}$. In this context, the transfer curves were measured to evaluate the efficiency of the reduction process. Figure 29b showed the output characteristics (drain current $I_{d}$ vs. drain voltage $V_{d}$ ) of the $\mathrm{SiO}_{2}$-gated graphene FETs at eleven different gate voltages $\left(\mathrm{V}_{\mathrm{g}}\right)$. 


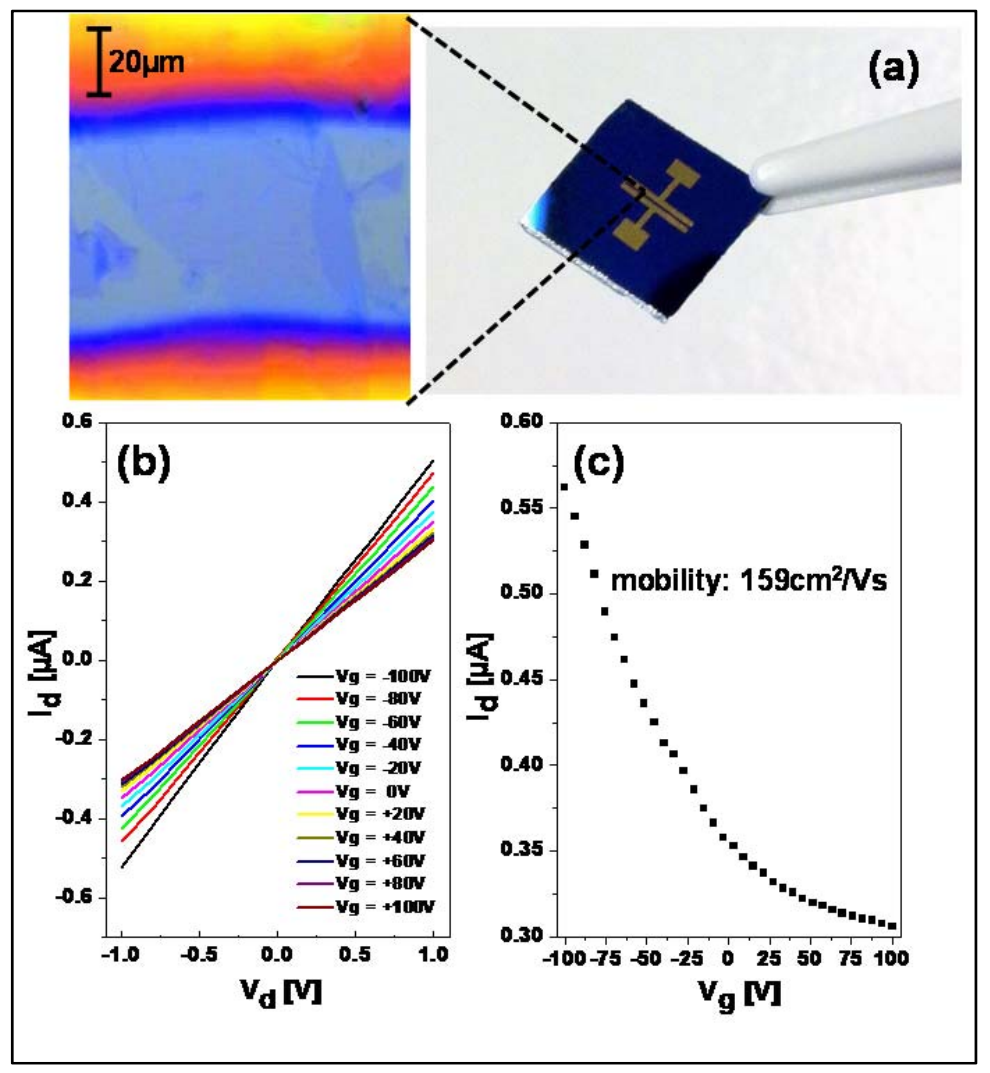

Figure 29: (a) Optical micrograph of the fabricated graphene device. (b) Output characteristics of the transistor device under different applied gate voltages. (c) Current voltage transfer curve of bottom-gated graphene FET at a drain-source bias of $V_{d}=0.1 \mathrm{~V}$

The device showed a clear increase in conductance as the gate voltage changed from $+100 \mathrm{~V}$ to $-100 \mathrm{~V}$, indicating that the RGO films behaved as p-type semiconducting materials ${ }^{248}$. Figure $29 \mathrm{c}$ demonstrated the typical transfer characteristics $\left(\mathrm{I}_{\mathrm{d}}-\mathrm{V}_{\mathrm{g}}\right)$ of the graphene field-effect transistor (FET) measured under ambient conditions (source-drain bias voltage $\mathrm{V}_{\mathrm{ds}}=100 \mathrm{mV}$ ) and its effective field effect hole mobility was $\sim 159 \mathrm{~cm}^{2} / \mathrm{Vs}$.

In order to extract the field-effect mobility of holes, only selected GO sheets with suitable size $(\sim 20 \mu \mathrm{m} \times 50 \mu \mathrm{m})$ were used for the transistor measurements ${ }^{13}$. Due to the indicated flake area, a graphene coverage corresponding to a channel width of $500 \mu \mathrm{m}$ was assumed. The channel width (W) and length (L) were 4000 and $60 \mu \mathrm{m}$, respectively. The hole mobility was calculated from the linear regime of the transfer characteristics using the 
equation $\mu=\left(L / W C_{o x} V_{d}\right)\left(\Delta I_{d} / \Delta V_{g}\right)$, where $\mathrm{L}$ and $\mathrm{W}$ were the channel length and width and $\mathrm{C}_{\mathrm{ox}}$ the gate capacitance.

Figure 30 summarized the distribution of the hole mobility of graphene FET arrays (total $\sim 75$ devices) on $\mathrm{SiO}_{2} / \mathrm{Si}$ substrate. A Gaussian fit indicated an average hole mobility of $40 \mathrm{~cm}^{2} / \mathrm{Vs}$, at a drain bias of $100 \mathrm{mV}$, which was remarkably higher than the reported ones after hydrazine chemical reduction ${ }^{13}$, 109. It was assumed, that by the improved graphene synthesis, the following hydrazine reduction process was carried out more effectively, leading to fieldeffect mobility values even comparable with those achieved with other reduction methods like alcohol vapour ${ }^{12}$.

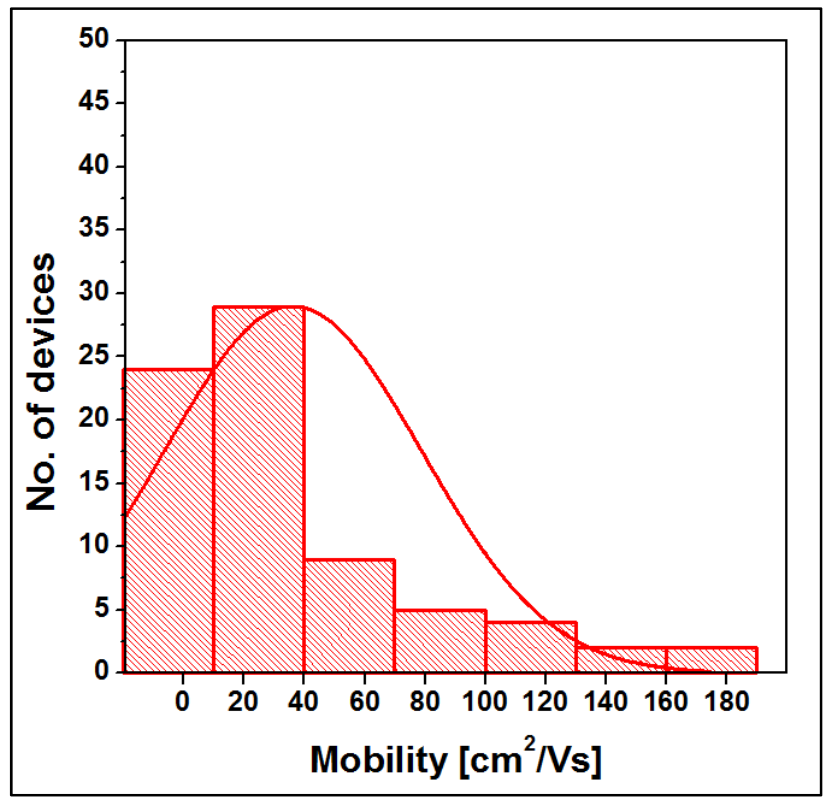

Figure 30: Distribution of the hole mobility of graphene FETs

Table 9 compared the effect of different reduction methods on the resistance of GO transparent electrodes. Although a similar transparency $(\mathrm{T}=95-98 \%$, corresponding to 1 or 2 layer $\mathrm{RGO}^{13}$ of the films were reported, the sheet resistance values vary notably. 


\begin{tabular}{lll}
\hline Process & Transparency & sheet resistance \\
& $@ 550 \mathrm{~nm}(\%)$ & $(\mathrm{k} \Omega / \square)$ \\
\hline hydrazine reduction & 98.3 & 145 \\
at $70{ }^{\circ} \mathrm{C}$ for $16 \mathrm{~h}($ reported method) & & \\
\hline hydrazine reduction & 95.4 & $19000^{251}$ \\
\hline hydrazine reduction at $80{ }^{\circ} \mathrm{C}$ & & $4000^{91}$ \\
\hline $20 \% \mathrm{H} 2 / \mathrm{Ar}, 1000{ }^{\circ} \mathrm{C}, 2 \mathrm{~h}$ & $\mathrm{~N} . \mathrm{d}$. & $188^{13}$ \\
\hline hydrazine reduction at $80^{\circ} \mathrm{C}$ for $24 \mathrm{~h}$ & $60-95$ & $100000^{13}$ \\
\hline
\end{tabular}

N.d. = not determined

The average sheet resistance of the RGO transparent electrode $(\mathrm{T} \sim 98 \%)$ (Figure 31) obtained by the reported modified method decreases dramatically to $\sim 145 \mathrm{k} \Omega / \square$, which was remarkable lower than the typical sheet resistance for hydrazine-reduced GO electrodes $245,248,251$. 


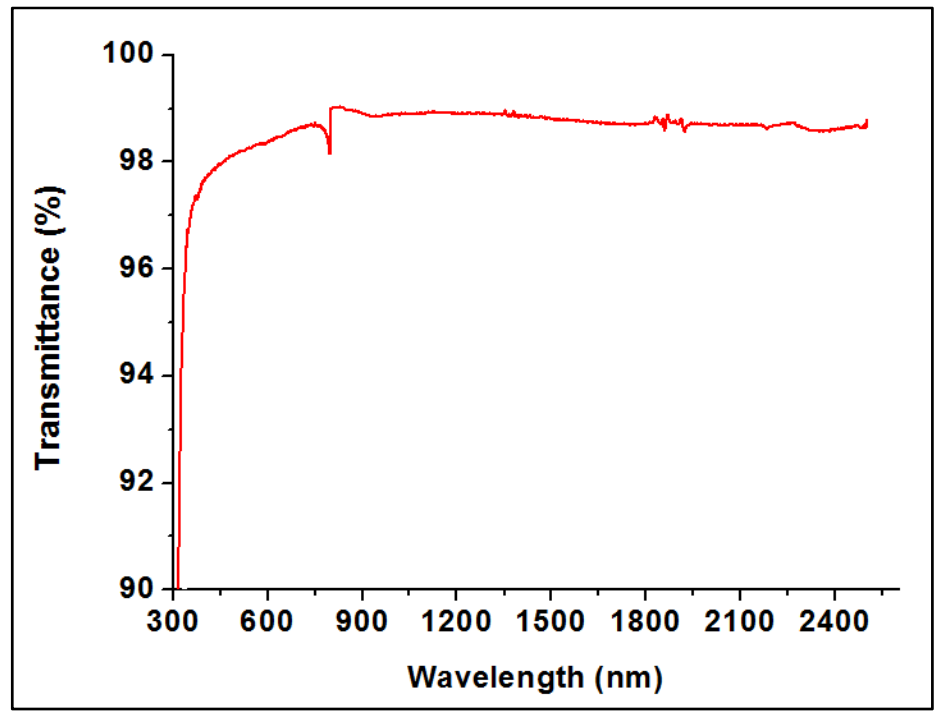

Figure 31: UV/vis-NIR spectrum of rGO

It was noted that the sheet resistance obtained without any additional annealing steps was already comparable (or even lower) with the $188-1005 \mathrm{k} \Omega / \square$ obtained by subsequent annealing at high temperature ${ }^{13}$. Furthermore, it should be mentioned that at least $23 \%$ of the 298 graphene devices tested exhibit sheet resistances significant lower than $100 \mathrm{k} \Omega / \square$ (Figure 32), which was previously obtained by more elaborate and time-consuming methods ${ }^{12,252}$.

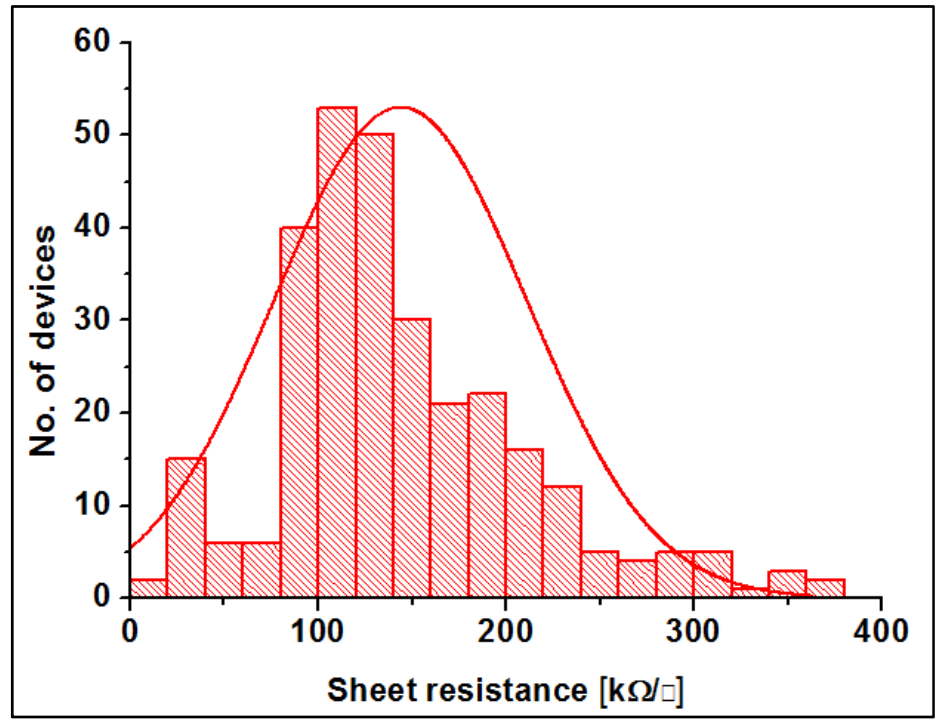

Figure 32: Distribution of the sheet resistance of graphene devices 


\section{$\underline{\text { 3.5 CONCLUSION }}$}

This chapter reported the new method to obtain large sized flakes of average $\sim 700 \mu \mathrm{m}^{2}$ with hole mobility averaging $40 \mathrm{~cm}^{2} / \mathrm{Vs}$. This was by far the largest size and highest hole mobility recorded from aqueous graphene without further processing or doping. The sheet resistance obtained without any additional annealing steps was comparable or lower than those obtained by subsequent annealing at high temperature. The chapter then reported the mechanism of large sized flakes via in-situ size fractionation. By using a pre-oxidation step with physical size fractionation and natural $\mathrm{pH}$ exfoliation, large sized flakes was obtained and this provided an improved raw material to high-performance and low-voltage operating graphene devices applicable in the field of biosensors described in the later chapters. 


\section{CHAPTER 4: DEVELOPMENT OF A}

\section{Disposable, All ORGANic Biosensor}

\section{WITH INTEGRATED PUMP FOR SODIUM}

\section{DETECTION}

This chapter described the development, fabrication and characterisation of a novel disposable pump integrated biosensor platform for the sensitive and selective detection of sodium. The integration allowed the sensor platform built to be small, portable and wearable; thus allowing the potential advantage to interface with the human body during field exercises enabling the provision of real-time data for immediate intervention. The sodium sensor was able to detect sodium ions from $10 \mathrm{mM}$ to $90 \mathrm{mM}$, within the typical physiological range for sweat sodium levels.

\section{$\underline{4.1 \text { INTRODUCTION }}$}

During exercise, sweating could lead to the loss of electrolytes such as sodium and potassium ${ }^{143}$. With increasing sweat rate, only $\mathrm{Na}^{+}$and $\mathrm{Cl}^{-}$(from extracellular compartments) concentrations in sweat tend to increase while $\mathrm{Ca}^{2+}$ (from intracellular space) decreases; $\mathrm{K}^{+}$and $\mathrm{Mg}^{2+}$ (from intracellular space) remain unchanged $^{144}$. Electrolyte loss through urine during exercise was small because of decreased urine formation and increased renal $\mathrm{Na}^{+}$reabsorption. Deficits of $5 \%$ to $7 \%$ of the body's $\mathrm{Na}^{+}$and $\mathrm{Cl}^{-}$ions could be lost through sweat in comparison to less than $1.2 \%$ loss for $\mathrm{K}^{+}$and $\mathrm{Mg}^{2+}$. ${ }^{144}$ Sodium levels in sweat could be used to ensure adequate $\mathrm{Na}^{+}$replenishment in order to prevent exercise-associated hyponatremia. Therefore, sodium measurement in sweat was important and essential for hydration status and was commercially important for the production of rehydration drinks. In this chapter, the characterisation, synthesis and sensing data of a novel disposable, organic, low- 
cost, graphene-based and pump-integrated sodium sensor suitable for real-time sensing of sweat sodium concentration in field conditions was presented.

\subsection{IDEA OF FULLY ORGANIC SODIUM SENSOR}

From a list of currently available sodium portable sensors (Table 6), there was a niche to develop a disposable and low-cost sodium sensor suitable for real-time sensing in field conditions. There was a demand for such a sensor platform in the fast-growing biosensor market which was projected to reach US\$ 18.9 billion globally in 2019, propelled by the growing population and health issues $^{253}$.

Increasingly graphene was the transducer material of choice because of its ability to detect single molecule binding events ${ }^{4}$. This was due to its exceptional low-noise electronic material property. Graphene, being a 2dimensional material with high surface area to volume ratio, was also very sensitive to small perturbations on its surface and these perturbations change its electrical resistivity drastically ${ }^{254}$.

Briefly, graphene was a single atom thick film made up of carbon atoms connected in a $\mathrm{sp}^{2}$ hybridized network. It had novel properties such as size tuneable band gap and high carrier mobility. Graphene had already demonstrated that it was able to detect single gas molecules ${ }^{4}$. Graphene can be obtained via several methods, including chemical vapour deposition ${ }^{255}$, mechanical exfoliation ${ }^{1}$ and chemical methods ${ }^{39}$. However, the chemical method had several advantages: low-cost, scalability and aqueous processability. Using graphene from the chemical route can lower the cost of sensors down to less than $£ 1$ per chip. Graphene produced via the chemical route contains several oxygen groups even after reduction and was thus sometimes termed reduced graphene oxide or reduced aqueous graphene ${ }^{256}$. RGO-based biosensors had also been reported to be able to detect single bacteria ${ }^{222}$ and cellactivities $^{11}$. The graphene transducer can be used in an optical, amperometrical or acoustical sensor setup. 
This chapter used the amperometric field effect transistor detection setup (Figure 33) as it could eliminate the need for expensive, bulky, highlyspecialised equipment in diagnostic screening tests. For selectivity, any receptors (e.g. sodium ionophore) could be attached to the amperometric graphene transducer to enable detection of a large variety of biological molecules with biomedical significance in real time.

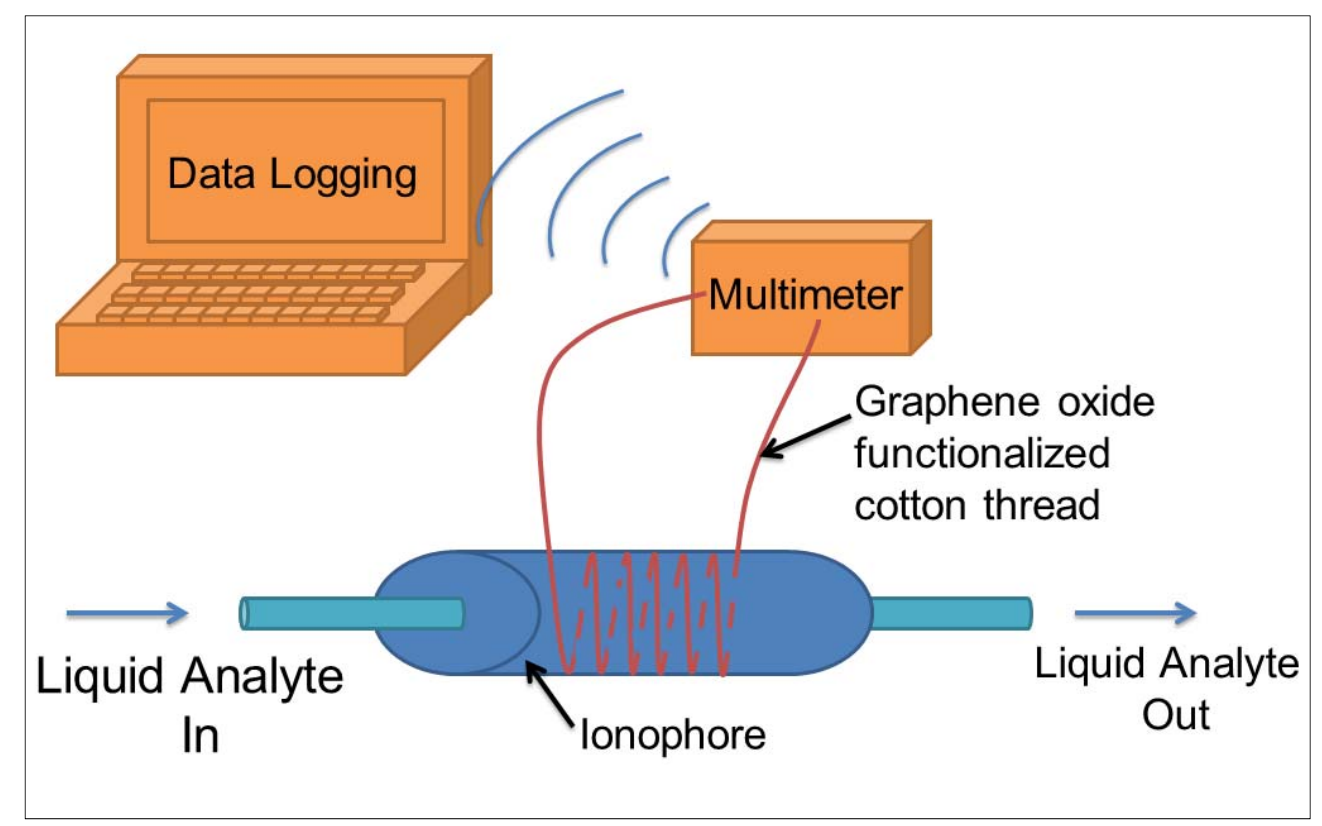

Figure 33: Schematic of amperometric field effect transistor (FET) biosensor setup

A proof of concept device using $\mathrm{rGO}$ on $\mathrm{SiO}_{2}$ with sodium ionophore was tested before the fabrication of the rGO on organic substrate. The device was rinsed with DI water $\left(0 \mathrm{mM} \mathrm{Na}^{+}\right)$at $180 \mathrm{~s}$ and $360 \mathrm{~s}$ to check for stability and then different concentrations of sodium ions were placed on the device at fixed time intervals. Thereafter, the device was rinsed two times at 1700s. The rinsing cycle consists of removing the previous analyte using cleanroom wipes and then replacing with fresh DI water.

From Figure 34a, it was observed that as the concentration of sodium ions increased, the current across the device decreased. It was noted that the recovery of the sodium sensor was not complete after two rinsing cycles. After a thorough rinse with running DI water, $90 \mathrm{mM}$ of $\mathrm{K}^{+}$was added to the device 
and this response was shown in Figure 34b. The response was negligible at a high concentration of $\mathrm{K}^{+}$, indicating good selectivity. The physiological concentration of $\mathrm{K}^{+}$was less than $10 \mathrm{mM}^{144}$.
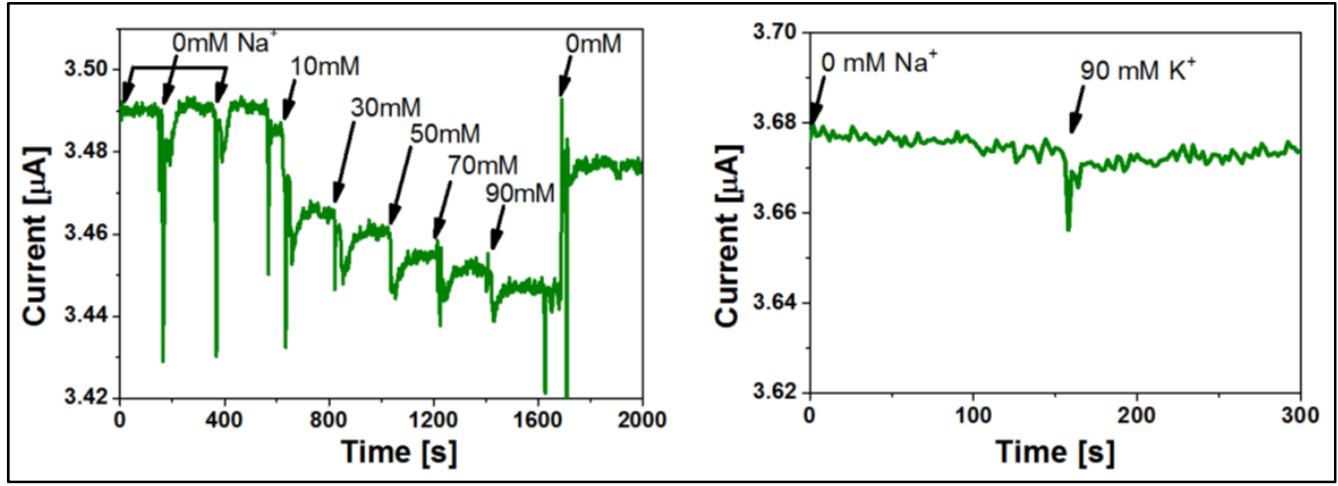

Figure 34: Electrical current output vs. sodium ion concentration input of the fully organic sodium sensor in response to (a) target sodium ions and (b) interference potassium ions.

\subsection{INTEGRATING SWEAT COLLECTION}

Methods for the collection of sweat include whole body washdown (WBW), which was the most accurate and reliable, with analysis undertaken later $^{257}$. However this method was limited to cycling and requires a controlled laboratory setting. Alternatively, regional skin collection was relatively simple, practical for field studies and was able to accurately and reliably predict WBW sodium concentrations ${ }^{149}$.

Regional skin collection methods with occlusive coverings had been reported to result in falsely high electrolyte readings because of electrolyte leeching from the stratum corneum of the $\operatorname{skin}^{151}$. However, if the skin can be kept dry by a sweat wicking material, reliable estimates of local sweat electrolyte concentrations could be obtained. A wicking sensor could thus prevent reabsorption of $\mathrm{Na}^{+}$back into the body. It would then be possible to measure the true $\mathrm{Na}^{+}$excretion. After these considerations, a novel rGO-based, amperometric sensor platform with an integrated pump which could be used for real-time sensitive detection of sodium ions was designed. 


\subsection{FABRICATION AND CHARACTERISATIONS}

Ultra-large Graphene Oxide (GO) flakes, a precursor to rGO, were prepared by modified Hummer's method from natural graphite flakes (3-5mm). Briefly, $2 \mathrm{~g}$ of graphite flakes were mixed with $\mathrm{H}_{2} \mathrm{SO}_{4}(12 \mathrm{ml})$ and stirred for 5 hours at $80^{\circ} \mathrm{C}$. The mixture was ultra-sonicated for 1 hour and diluted with DI water $(500 \mathrm{ml})$. The suspension was then filtered using $0.2 \mu \mathrm{m}$ filter to obtain dry preoxidized graphite powders. To fully oxidize the graphite, $\mathrm{H}_{2} \mathrm{SO}_{4}(120 \mathrm{ml})$ and $\mathrm{KMnO}_{4}(15 \mathrm{~g})$ was added to the powder and stirred for 2 hours before diluting with $950 \mathrm{ml}$ of DI water slowly in ice-bath and finally stopping the reaction using $\mathrm{H}_{2} \mathrm{O}_{2}(20 \mathrm{ml})$. The upper portion of the solution was collected, filtered and washed to remove remaining metal ions and acid. This forms the stock GO solution containing large GO flakes used in subsequent experiment.

To image the large GO flakes, GO solution was drop-cast on silicon dioxide flat substrate and scanning electron microscopy was used (Figure 35a). Using this method, the average size of the GO flakes were $700 \mu \mathrm{m}^{2}$ and this was 7 orders of magnitude larger ${ }^{39}$ than existing literatures. Larger GO flakes exhibited remarkable lower sheet resistance, lower intra-flake resistance and higher hole carrier mobility which result in a more sensitive sensor.

To prepare the cotton substrate for the rGO, cotton threads were treated with oxygen $(25 \%)$ plasma. This treatment removed natural wax and increased oxygen moieties on the surface of the thread, making subsequent chemical functionalization possible. After a 50 minute plasma treatment, the physical structure of the cotton thread was still intact (Figure 35b). Then (3Aminopropyl) triethoxysilane $(2 \% \mathrm{v} / \mathrm{v}$ ethanol) was used to functionalise the cotton surface to make it positively charged. The functionalised thread was then submerged in the GO stock solution for 30 minutes. As the surface of GO was negatively charged, during the incubation period, the GO would be electrostatically attached onto the thread.

It had been reported that GO could also be coated directly onto the cotton thread using a drip-and-dry method ${ }^{258}$. However, attaching the graphene using the electrostatic attraction method allowed self-assembly and termination, thus 
allowing controlled 1-2 layers of GO to be adhered to the thread (Figure 35c). The 2-dimensional material characteristics of GO was preserved with 1-2 layers of GO as the hole or electrons carriers could only move along the planar direction. Having more than two layers of GO would allow the carriers to travel in parallel (3-dimensional) and decrease the sensor's sensitivity to any perturbations from the ionophore.

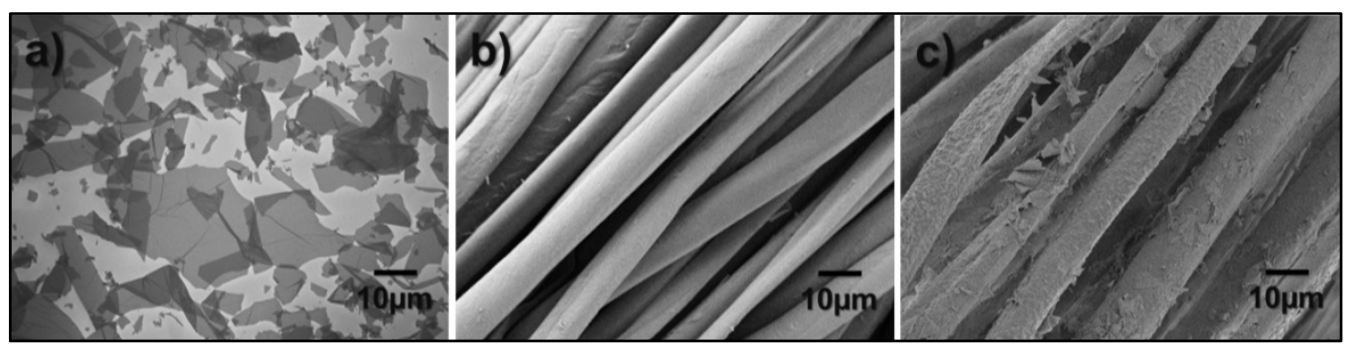

Figure 35: Field emission scanning electron microscopy images of (a) graphene oxide flakes on silicon dioxide wafer (b) cotton thread after plasma treatment (c) graphene oxide on cotton thread taken under similar magnification

After the attachment of GO to the thread, a chemical reduction was needed to remove oxygen moieties from the carbon backbone of $\mathrm{GO}$ and restore its electrical conductivity. The thread was placed in a sealed petri dish with $500 \mu l$ of hydrazine monohydrate and left overnight at $70^{\circ} \mathrm{C}$. The hydrazine vapor produced will reduce the GO into rGO. This rGO-lined cotton thread formed the rGO transducer. The exposed rGO transducer was sensitive to all molecules, such as $\mathrm{H}_{2} \mathrm{O}$ (humidity) and $\mathrm{NO}_{2}$ (gas) molecules. Therefore a coat was needed to protect the rGO transducer from the environment.

To achieve selectivity of sodium on the rGO surface, a sodium ionophore cocktail was coated onto the rGO transducer. Briefly, bis(1-butylpentyl) adipate, sodium ionophore (71733) and poly(vinyl chloride) high molecular weight, (Sigma-Aldrich, St Louis, USA), were dissolved in tetrahydrofuran solvent and then the ionophore cocktail was left overnight to stabilize before coating on the rGO transducer. The ionophore used was an electrically neutral, lipophilic ioncomplexing agent of small relative molar mass. The rGO transducer was dipped 
into the sodium ionophore two times to ensure complete coverage so that the liquid analyte cannot seep into the transducer. After drying, the rGO transducer was then dipped into coloured dye to check for staining and to ensure that the rGO transducer was water-tight.

The ionophore-covered rGO transducer was then fixed across a polyethylene terephthalate (PET) platform and attached to a multimeter (Agilent U1273A and E3ABAG) via copper wire and clips. Another plasma-treated thread was coiled around the rGO transducer. This allowed the liquid analyte to flow through it to reach the transducer (Figure 36). One of the thread ends was attached to a silicone rubber liquid analyte inlet and the other to a cotton wool excess liquid analyte reservoir. The large collection reservoir allowed constant flow of liquid analyte through the thread. This thread acted as a pump using capillary action and no external power source was needed ${ }^{259,260}$. After the sensing setup was completed, coloured dyes were used to detect the movement of liquid analyte.

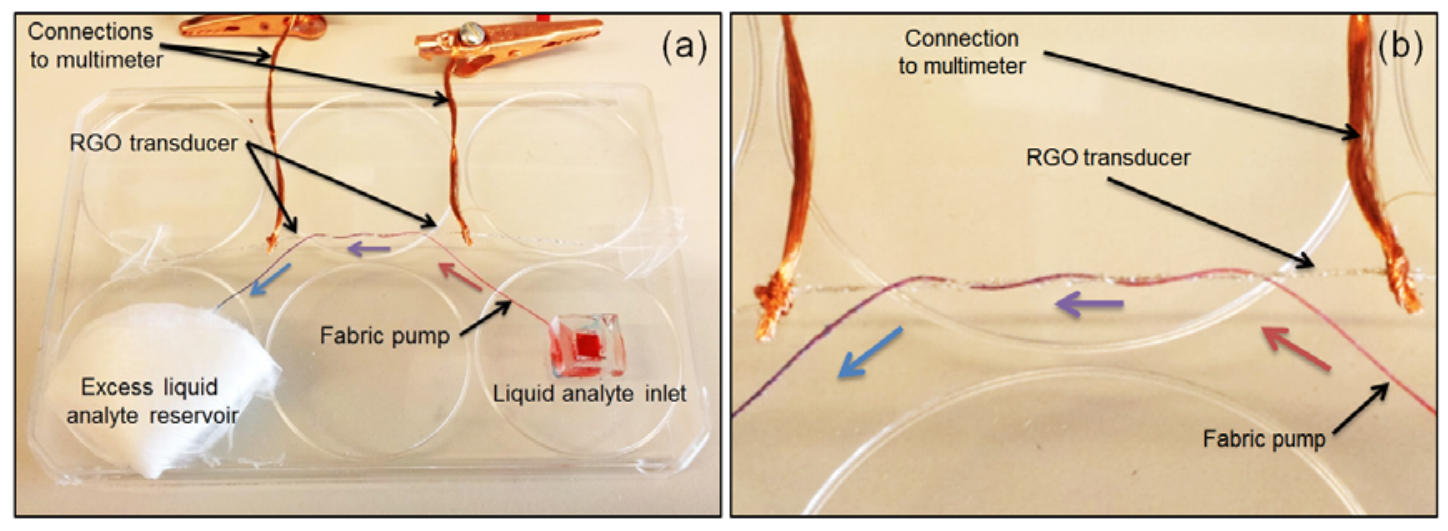

Figure 36: (a) Graphene oxide based sensor with integrated fabric pump. (b) A zoomed in image of the RGO transducer in the sensor. 


\subsection{PUMP TESTING AND SODIUM SENSING}

From Figure 36, it could be observed that the blue and then red coloured dyes travelled cleanly along the fabric pump. The wicking rate increased exponentially with increasing plasma treatment time on the cotton thread ca. $3 \mathrm{~mm} / \mathrm{min}, 10 \mathrm{~mm} / \mathrm{min}$ and $50 \mathrm{~mm} / \mathrm{min}$ after $10,30,50$ minutes of oxygen plasma treatment respectively. In the experiments, 50 minutes of plasma treatment on all cotton thread was used. Then the coloured dyes were replaced with sodium analyte for sensing data collection.

To test the sodium detection window of the sensor, different concentrations of sodium chloride solution were added to fill the analyte inlet and allowed to flow through the thread pump. The liquid analyte travelled via capillary action along the thread pump towards the ionophore-coated transducer. The ionophores selectively extract sodium ions into the hydrophobic membrane phase and transport these ions across the barrier by carrier translocation. The ions carried across the ionophore then cause perturbations on the rGO surface and change the electrical resistivity. This change in electrical property could be detected by a multimeter and the data was then sent to a data-logger. A sensing window was thus obtained and presented in Figure 37.

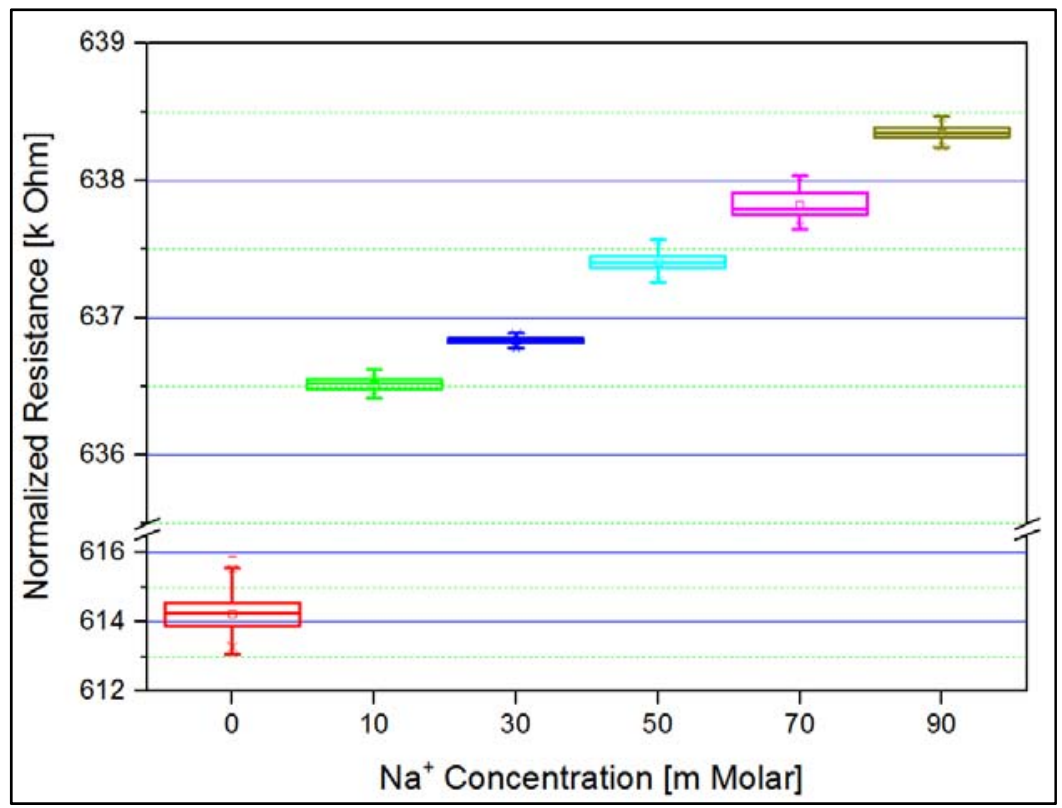

Figure 37: Electrical resistance output vs. sodium ion concentration input of the fully organic sodium sensor 
As the fabricated $\mathrm{rGO}$ transducer was a p-type semiconductor ${ }^{261}$, when positive sodium ions travel through the soium ionophore onto the surface, it induced a positive gating effect ${ }^{262}$ and increased the rGO's electrical resistance. The electrical resistance changed according to the change in the concentrate on of sodium chloride.

As the concentration of the sodium chloride increased from $0 \mathrm{mM}$ to $90 \mathrm{mM}$, the electrical resistance through the $\mathrm{rGO}$ transducer increased from 615.2 to $638.5 \mathrm{k} \Omega$ correspondingly. A $90 \mathrm{mM}$ sodium ion concentration contributed a $3.9 \%$ increase in electrical resistance across the rGO transducer.

Patterson et al ${ }^{149}$ reported whole-body sweat $\mathrm{Na}^{+}$concentrations of $24.1 \pm 15.0$ $\mathrm{mM}$ (mean $\pm \mathrm{SD}$ ) from ten males cycling on a friction-braked ergometer for 90 $\min$ at $20^{\circ} \mathrm{C}$. The sodium levels at specific regions such as the forearm, chest and scapula were $42.2 \pm 25.8,47.6 \pm 25.7$ and $42.2 \pm 24.8 \mathrm{mM}$ respectively. Therefore, the reported sensor operated within the typical physiological range. As can be seen from the large standard deviations for sweat $\mathrm{Na}^{+}$levels, sweat composition could vary widely between individuals.

In theory, the lower detection window of the reported transducer was only limited to the sodium ionophore applied and electronic amplification equipment used, as graphene had already been shown to detect 1 single molecular binding events ${ }^{4}$. As a comparison, a single litre of $1 \mathrm{M}$ of sodium chloride solution contains $6.023 \times 10^{23}$ number of sodium ions. With such high sensitivity, this rGO-based sensor could also be modified with antibodies or other ionophores to detect other specific ions or biomolecules of interest to the community. The presented sodium sensor could potentially be used to diagnose dehydration in the ageing population and used in the study of the biology of sweat in older people. Future work on the disposable and low-cost real-time fully-organic sensor platform include optimizing the fabrication technique for massproduction and sensing for other ions or proteins. 


\subsection{CONCLUSION}

During exercise, one of the body's major ionic deficits of concern was $\mathrm{Na}^{+}$. However, there were no portable real-time sodium sensors suitable for field testing. This chapter reported the fabrication, characterization and physiologically-relevant sodium-ions detection limits of a novel rGO-based sensor with an integrated pump system that was fully organic, low-cost and disposable. This prototype has potential for small, light wearable sensor. It had enormous potential to be integrated onto the human body during field training to obtain real-time data for immediate intervention. 


\section{Chapter 5: NOVEl CARbon Nanotube- \\ GRAPHENE OXIDE HYBRID STRUCTURE}

AND ITS MECHANISM

This chapter described the novel fabrication technique and mechanism to obtain a new CNT-rGO hybrid structure on which the rGO coverage could be controlled. The hybrid layer of CNT-rGO-CNT exhibited low electrical resistivity $(60 \Omega / \square)$ at high optical transparency $(94.7 \%)$ and immense degree of wrinkled surfaces $(\sim 20 \%)$ on the rGO surface. These findings had practical implications for the fabrication of highly-tuneable novel electronics and biosensors.

\subsection{INTRODUCTION}

A new fabrication method and a new structure were described in this chapter. The novel method yielded the formation of a novel pristine Carbon Nano-Tubes (CNT) with mono-layered Graphene Oxide (GO) hybrid film structure. The resultant pristine-CNT-GO layered film was a new structure that had not been reported before. This method required no additional chemical modification, growth or sonication steps which render the hybrid film non-pristine and degrade its electrical properties. In this technique, the CNT and GO layers were self-assembled. One fabricated sandwich layer of the rGO-CNT-GO film exhibited very high electrical conductivity $(60 \Omega / \square)$ at high optical transparency $(94.7 \%)$ and immense degree of wrinkled surfaces (20\%) on the rGO surface. The method and the resulting novel hybrid structure were highly-tuneable, even in the nano-meter scale, and enabled novel and promising approaches for energy storage, solar cells, semiconductors and biosensors. 
Graphene and CNT share similarities such as mono-layered $\mathrm{sp}^{2}$ carbonhybridization carbon arrangement and high carrier mobility. Both had exceptional physical, mechanical, electrical and structural properties, thus attracting tremendous attention in different research fields such as photovoltaic, nano-electronics, super-capacitors, biosensors and transparent electrodes in recent years ${ }^{263}$.

However, graphene was a two-dimensional carbon material while Carbon Nano-Tube (CNT) was a one-dimensional material. Due to this dimensional difference, Graphene forms a layered thin film structure whereas CNT forms a networked structure. Both resultant structures contain many voids that limit the electrical conductivity and optical transparency. The electrical percolation threshold of graphene was lower than CNT; less amount of graphene (higher film transparency) was needed to reach conductive threshold level while CNT requires more amounts (low film transparency). However, graphene had higher inter-flake electrical resistance which can be decreased by introducing CNT. A future trend was to combine the two films into a hybrid material. There had been attempts to integrate the merits of both recently.

It had been reported that hybrid material of graphene with CNT could provide additional electron-transport path, enhance the DC conductivity without reducing optical transmittance (up to $100 \Omega / \square$ at $80 \%$ transparency) ${ }^{264}$, exhibit improved good rate performance, cycling properties in fast lithium storage $\left(121.8 \mathrm{~mA} \mathrm{~h} \mathrm{~g}^{-1} \text { at the rate of } 10 \mathrm{C} \text {, after } 100 \text { cycles with } 8.7 \% \text { capacity loss }\right)^{265}$ and super-capacitor performance $(120 \mathrm{~g} / \mathrm{F} \text { at scan rate of } 1 \mathrm{~V} / \mathrm{s})^{266}$. The $80 \%$ transparency reported ${ }^{264}$ was equivalent to having about 10 GO layers (monolayer rGO was known to have $2 \%$ absorption). These results were important as they show that hybrid materials can be improved upon. It had also been reported $^{267}$ that using GO film alone had already resulted in a $10^{2}-10^{3} \Omega / \square$ with $80 \%$ transparency. Thus the hypothesis was that by controlling hybrid film of CNT-GO, there will be a drastic increase in transparency and yet maintain a low electrical resistivity. A facile and manufacturing-scalable synthesis method to deliver such a hybrid material was of great importance. 
Firstly, approaches had been made to produce non-pristine CNT-GO films using electrostatic attraction with additional precursors to functionalize either the GO or the CNT. Both CNT and GO were negatively charged, therefore polyethylenimine and amine groups had been added to $\mathrm{GO}^{268}$ and $\mathrm{CNT}^{269,270}$ respectively, to make it positively charged. These additions of chemicals and functionalization needed increased the cost and degrade the performance of the thin film.

Then, attempts had been made to produce hybrid layers by directly growing the layers alternatively and individually in CVDs using cobalt-based catalysts ${ }^{271}$. This process was not only tedious and limited in growth area, it also introduced metal catalyst and amorphous carbon contaminants ${ }^{272}$ on the thin film surface.

Finally, attempts had been made to produce CNT-GO meshed films by sonicating CNT and GO solutions together into a homogeneous solution followed by re-deposition them ${ }^{264,273-277}$. However, this ultra-sonication induced stress in the GO flakes and CNTs thus breaking them into smaller pieces, increasing surface defects and reducing conductivity. Furthermore these resulting films were not structured and were too thick for optical applications ${ }^{275}$.

This was the first report to obtain a transparent conducting thin film of CNT with mono-layered GO from as-synthesized GO and CNT, without any need for chemical modification, growth step or sonication steps. This important observation enabled a simple in-situ reduction and attachment of GO to CNT film, for extensive industrial applications in various areas, with excellent stability in atmosphere and in aqueous environment. This simple method also reduced experimental parameters. In the next section, the method and characterization of the highly conductive and transparent structure will be presented. 


\subsection{FABRICATION OF CNT-GO HYBRID STRUCTURE}

Silicon substrates (500nm thermally-grown silicon oxide layer) of $1.5 \mathrm{~cm} \times 1.5$ $\mathrm{cm}$ were first cleaned with a standard RCA solution to remove undesired impurities. Subsequently, the cleaned $\mathrm{SiO}_{2}$ substrates were modified with 3aminopropyl triethoxysilane (APTES) self-assembled monolayers (SAMs). The substrates were immersed into a $1 \%$ APTES solution in ethanol for $1 \mathrm{~h}$, followed by extensively rinsing with absolute ethanol, before baking for 2 hours at $120{ }^{\circ} \mathrm{C}$. Ultra-large GO flakes were prepared using the modified Hummer's method described in earlier chapters.

Carbon Nano-Tube (Puretubes, Nanointegris) was obtained commercially without further purification. CNT solution was drop-cast onto the devices for 1 hour, followed by thoroughly rinsing with DI water and drying with $\mathrm{N}_{2}$. SEM images of the CNT were shown in Figure 38.

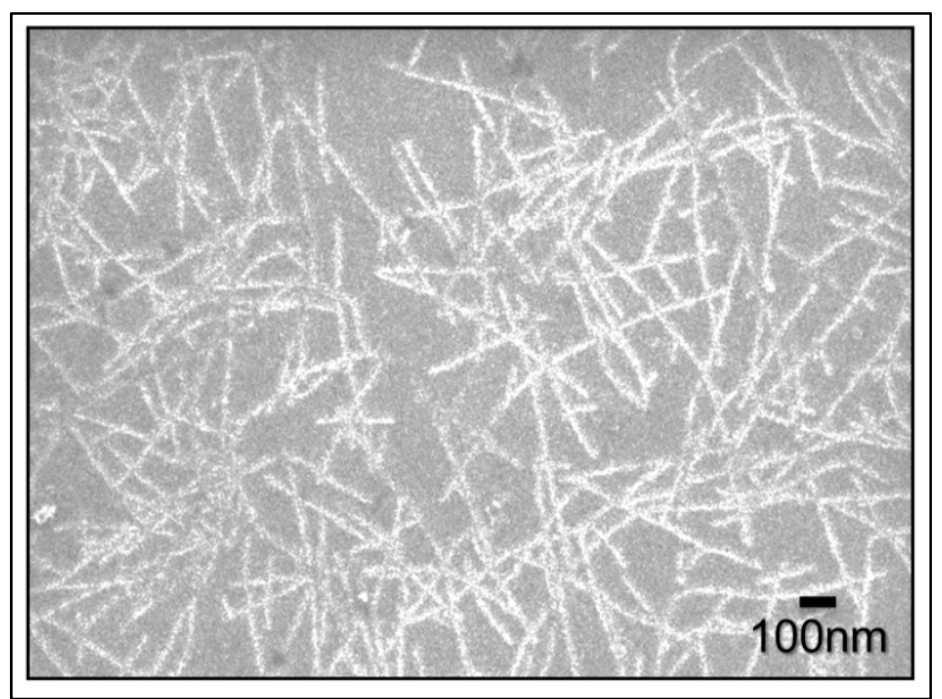

Figure 38: SEM image of self-terminating carbon nanotubes (CNT) on functionalized $\mathrm{SiO}_{2}$ substrate

Thereafter, it was placed in a freshly RCA-cleaned glass-beaker with $50 \mathrm{ml}$ of GO solution. The glass beaker was placed under reflux and heated to $100^{\circ} \mathrm{C}$. After different deposition times, the chips were removed and rinsed with copious DI water to remove unattached GO flakes. 
To reduce usage of the extremely toxic hydrazine, hydrazine vapor was used instead of pure hydrazine. In this reduction, samples were placed into glass petri dishes and $500 \mu 1$ of $98 \%$ hydrazine monohydrate (Sigma) was dropped in the centre of the petri dish. The samples were kept in the sealed petri dish at $70{ }^{\circ} \mathrm{C}$ for 16 hours.

\subsection{CHARACTERIZATION AND DISCUSSION OF CNT-GO HYBRID STRUCTURE}

The schematic illustration of the CNT-GO layers was shown in Figure 39. The silicon substrates were RCA-cleaned, then modified with 3-APTES. Thereafter CNT were drop-cast on the surface and rinsed. The chip was then placed in GO solution under reflux with various times and then rinsed. The GO solution must be contained in a freshly-cleaned RCA-washed glass crystallization dish (hydrophilic) to prevent undesirable adhesion of the GO. As the GO was gently heated up in the aqueous solution, it changed from hydrophilic to hydrophobic. Due to the increasing hydrophobic nature of GO, it will preferentially attach itself to the hydrophobic CNT via to $\pi-\pi$ stacking between $\mathrm{sp}^{2-}$ hybridised carbon areas. 


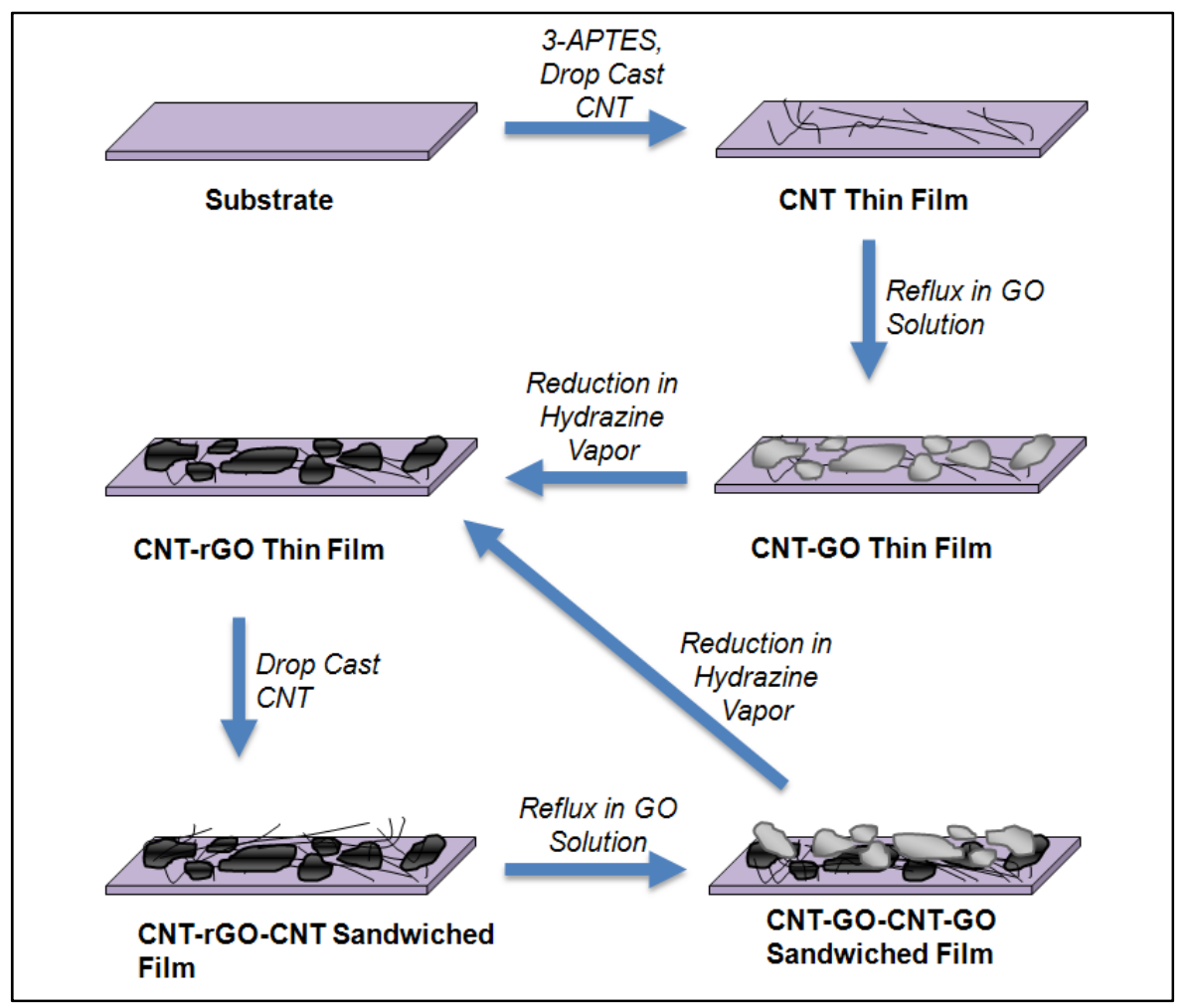

Figure 39: Schematic illustration of the fabrication of the CNT-GO layers

The $\pi-\pi$ stacking enabled a network of pristine CNT network with GO on top to form without any chemical additives. This method could be repeated to obtain a sandwiched layer as shown in Figure 40.

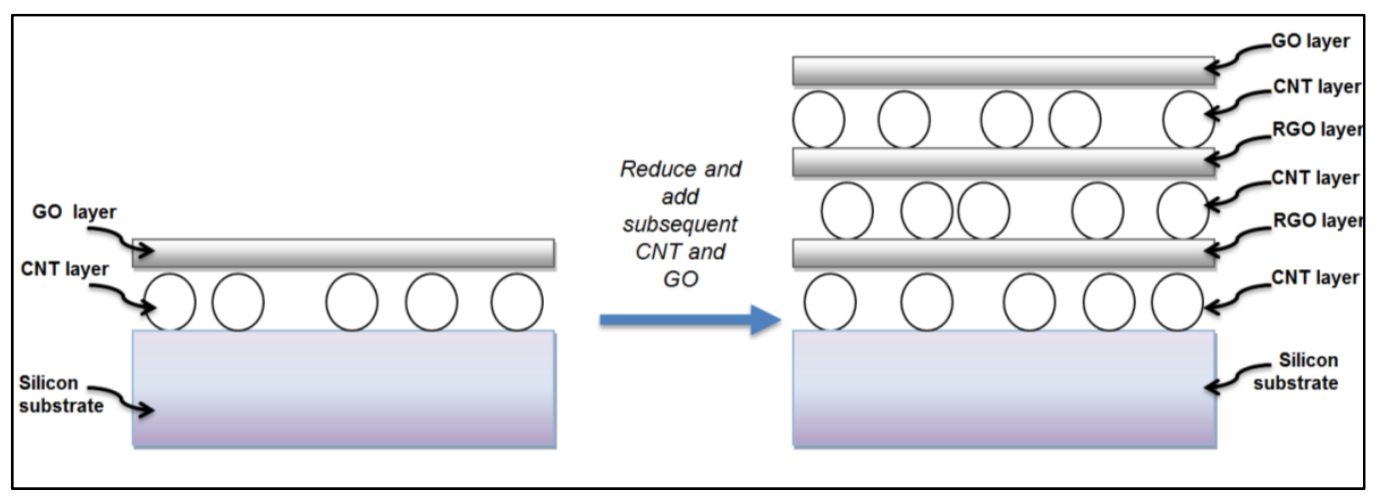

Figure 40: Schematic of CNT-GO layers and its sandwich structure 
A time-temperature study was conducted, Figure 41, and it showed that the coverage of GO was controlled by reflux time. Increased processing time led to an increased coverage of GO. After a refluxing at $100^{\circ} \mathrm{C}$ of 1 hour, a monolayered and spaced out GO coverage of ca. 20\% was obtained (Figure 41a). After 3 hours, the coverage of the GO increased to $60 \%$ (Figure $41 \mathrm{~b}$ ). At 5 hours, the coverage was $85 \%$ and the GO sheets formed percolated networks (Figure $41 \mathrm{c}$ ). At the end of 9 hours reflux, the GO coverage was 95\% and the flakes were densely packed (Figure 41d). Even after 9 hours, the hybrid sheet consisted of only 1-2 layers of GO sheets and the GO assembly self-terminate. The temperature in the reaction vessel was $92{ }^{\circ} \mathrm{C}$.

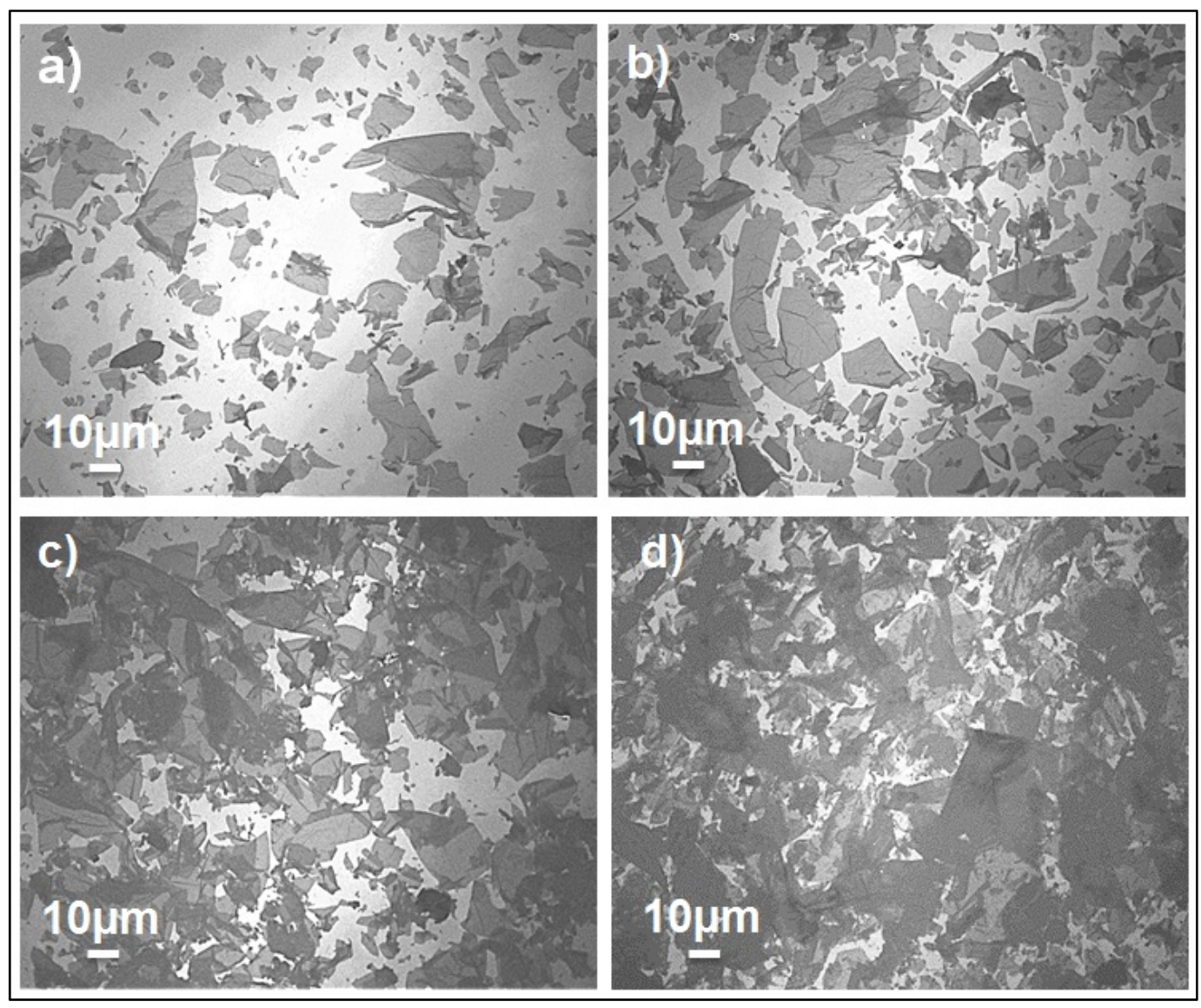

Figure 41: Time dependent study of GO coverage on CNT under constant reflux of a) 1, b), 3, c) 5 and d) 9 hours 
In a control experiment, CNT-coated silicon chips were submerged in GO solution at room temperature for 9 hours. The GO and CNT did not attach to each other at room temperature even after 9 hours (Figure 42); this was due to the electrostatic repulsion force by GO's oxygen moieties.

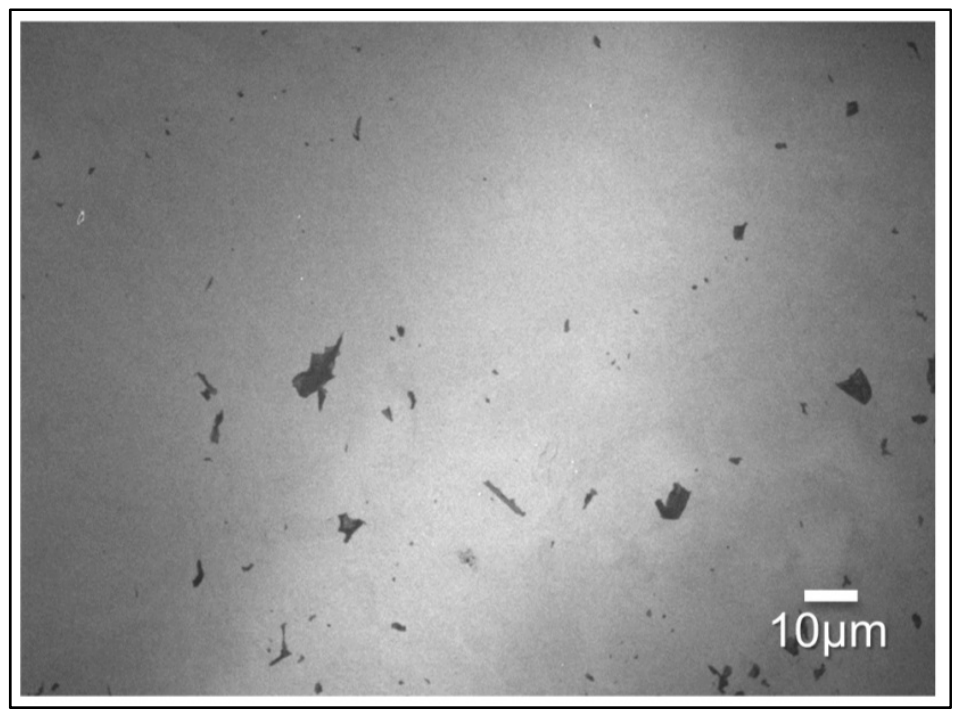

Figure 42: SEM image of control experiment conducted with GO and CNT at room temperature after 9 hours.

The stock GO solution was shown in Figure 43a. The GO solution with $\mathrm{NaCl}$ added (Figure 43b) had mild agglomeration and the GO did not form a hybrid layer with the CNT. For alkaline condition (Figure 43c), the GO agglomerated together and not adhere on the surface of the CNT. This was unexpected since it was reported that alkaline was similar to a thermal reduction ${ }^{103}$. The observation was in agreement that alkaline conditions only removed preferential hydroxyl groups over epoxides ${ }^{278}$. Thus it is proposed that after the partial deoxygenation of alkaline reduction, the easily removed hydroxyl groups from the $\mathrm{GO}$ were removed leaving the more stubborn epoxide functionalities. These proposed epoxide functionalities could continue to prevent the adhesion of the GO to the surface of the CNT but not the agglomeration amongst GO. Thus $\mathrm{pH}$ and temperature (Figure 43d) could be used for the optimization of the reported process. 


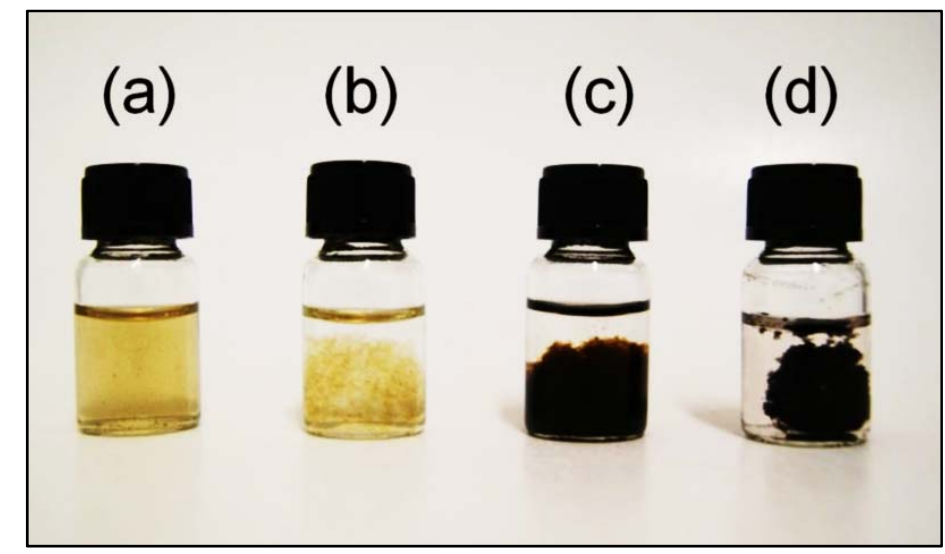

Figure 43: Photographic image of a) GO solution b) GO with $\mathrm{NaCl}$ c) GO with $\mathrm{NaOH}$ d) $\mathrm{NaOH}$ with 3 hour reflux

As the GO reflux in the aqueous solution, it changed from hydrophilic to hydrophobic. This mild reduction ${ }^{249}$ of GO after 1 hour reflux to a more hydrophobic GO could be observed in the Raman spectra as the D/G intensity was reduced from 0.97 to 0.92 .

Since CNT has a hydrophobic nature ${ }^{279}$, during reflux of the $\mathrm{CNT}-\mathrm{SiO}_{2}$ chips in GO solution, the mildly reduced GO will attach itself to the CNT and form a network of pristine CNT network with GO on top, held together by noncovalent $\pi-\pi$ interaction without any chemical additives.

It was observed that the deposited smaller flakes were not covered by larger ones on all obtained chips (Figure 44). This required the larger GO flakes to be deposited first onto the CNT thin film, followed by smaller GO flakes. In agreement to Lerf-Klinowski's GO model in which smaller GO possess persistent high charge density, especially at the edges than larger GO, the larger GO sheet with its larger basal planes lost more oxygen functionalities than the smaller GO sheet during mild deoxygenation leading to the preferential deposition of larger flakes. 


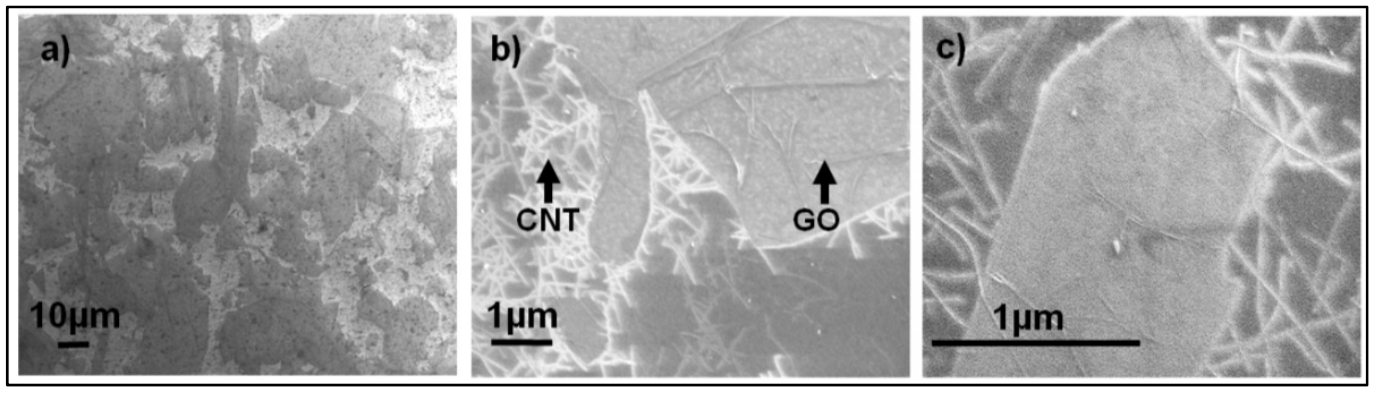

Figure 44: SEM images with $1 \mathrm{keV}$ showing CNT-GO hybrid layer at a) 800x, b) 16000x and 55000x magnification

This reported method also produced highly wrinkled surface (Figure 45) on substrate that had not been reproduced or reported elsewhere. Increased wrinkling allowed more sites for DNA and proteins to bind ${ }^{54}$ thus increasing surface loading for applications such as bio-sensing transducer.

The Scanning Electron Microscope images of CNT and CNT-GO film were shown in Figure 45. Figure 45a showed a mono-layer of GO on top of a thin layer of CNT. Figure 45b showed the wrinkling effect observed on the GO and these wrinkles conformed to the CNT network underneath. The wrinkles increased as the reflux process time was increased, indicating that the wrinkle formation was due to thermal-stress around CNT networks. At these wrinkled locations, there was high curvature of the graphene leading to increased strain energy induced by the local mechanical deformation. These wrinkles had been reported to exhibit enhanced chemical reactivity to radicals ${ }^{280}$, electrical conductivity $^{281}$, promotion of spontaneous stem-cell differentiation towards bone cell linage ${ }^{282}$, faster bridge for electron transport ${ }^{283}$, increased DNA surface loading ${ }^{54}$ and could also be employed for graphene nano-ribbon formations ${ }^{284}$. 


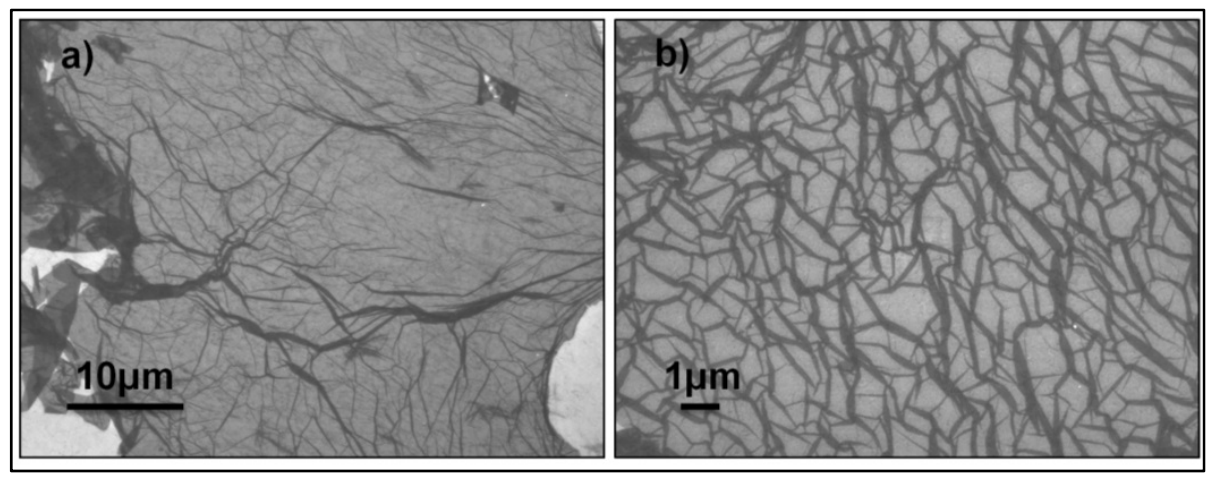

Figure 45: SEM image of a) heavily wrinkling b) and its higher magnification on rGO surface after 5 hours reflux. The wrinkling accounted for $20 \%$ of surface area and was interconnected

Thermal gravitational analysis of freeze-dried GO (Figure 46), showed that 18 $\%$ of the freeze-dried $\mathrm{GO}$ weight was lost even at a mild $100^{\circ} \mathrm{C}$ heating. As GO had high entropy and a possible lower stable state, a low temperature annealing could decompose the labile oxygen groups, leading to the observed weight loss. This important observation enabled a simple in-situ reduction and attachment of GO to CNT film, for extensive industrial applications in various areas, with excellent stability in atmosphere and in aqueous environment. This simple method also reduced experimental procedures and parameters. A comparison of the reported CNT-rGO hybrid film to existing hybrid films was presented in Table 10.

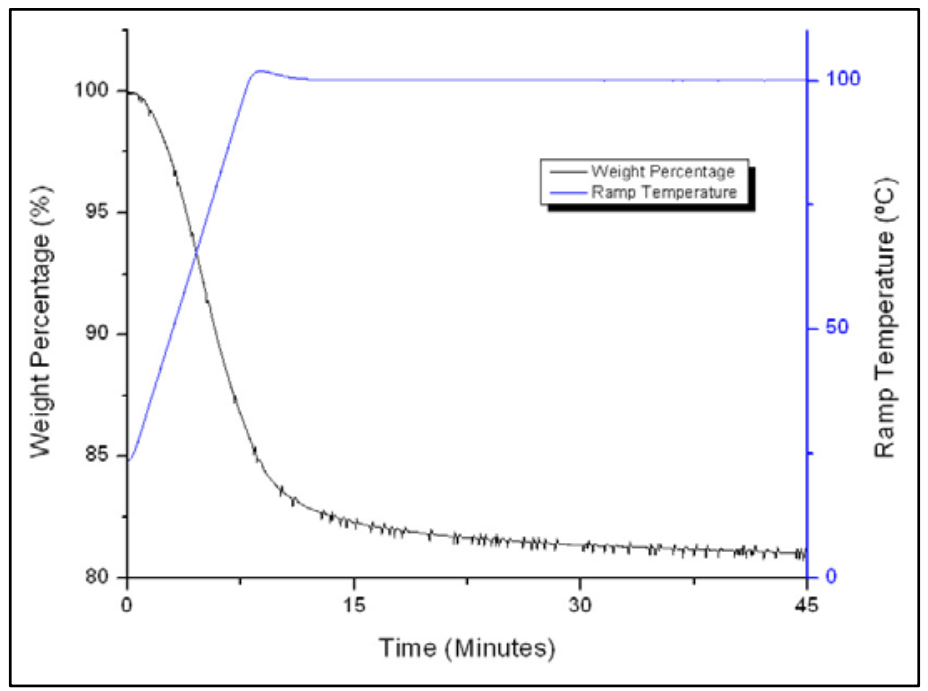

Figure 46: TGA of freeze-dried $\mathrm{GO}$ at $100^{\circ} \mathrm{C}$ 
Table 10: Comparison of existing CNT-rGO hybrid film methods

\begin{tabular}{|c|c|c|c|c|}
\hline & Sonication & Growth & $\begin{array}{c}\text { Chemical } \\
\text { Modification }\end{array}$ & $\begin{array}{l}\text { Reflux Treatment } \\
\text { (Reported Method) }\end{array}$ \\
\hline Description & $\begin{array}{l}\text { Sonicating CNT } \\
\text { and GO together }\end{array}$ & $\begin{array}{l}\text { Grow Graphene } \\
\text { layer on CNT or } \\
\text { vice versa }\end{array}$ & $\begin{array}{c}\text { Chemical synthesis } \\
\text { to make CNT and } \\
\text { GO oppositely } \\
\text { charged }\end{array}$ & Reflux in GO solution \\
\hline $\begin{array}{l}\text { Mechanism of } \\
\text { Attraction }\end{array}$ & Van der Waals & Van der Waals & Electrostatic & Van der Waals \\
\hline Precursors & NIL & $\begin{array}{c}\text { Cobalt-based } \\
\text { catalyst }\end{array}$ & $\begin{array}{l}\text { Amides, oxidizing } \\
\text { agent etc. }\end{array}$ & NIL \\
\hline Time & Short & Long & Medium & Short \\
\hline Cost & $\begin{array}{l}\text { Low (Ultrasonic } \\
\text { machine) }\end{array}$ & $\begin{array}{l}\text { Very high cost } \\
\text { (CVD equipment, } \\
\text { gas setup). Low } \\
\text { vacuum required. }\end{array}$ & $\begin{array}{l}\text { Medium cost } \\
\text { (Reagent Grad } \\
\text { Chemicals) }\end{array}$ & $\begin{array}{l}\text { Very low (Beaker, } \\
\text { Liedberg condenser } \\
\text { and heating plate) }\end{array}$ \\
\hline $\begin{array}{c}\text { Main } \\
\text { disadvantage }\end{array}$ & $\begin{array}{l}\text { Damages } \\
\mathrm{CNT} / \mathrm{GO} \\
\text { structure }\end{array}$ & $\begin{array}{l}\text { Metal catalyst } \\
\text { used remains in } \\
\text { the film }\end{array}$ & $\begin{array}{l}\text { Additional chemical } \\
\text { functional groups } \\
\text { were added to the } \\
\text { film. }\end{array}$ & $\begin{array}{l}\text { Process was self- } \\
\text { terminating, only } 1 \\
\text { GO layer formed }\end{array}$ \\
\hline Main advantage & Simple & Self-terminating & Self-terminating & $\begin{array}{l}\text { Simple and self- } \\
\text { terminating }\end{array}$ \\
\hline Product & $\begin{array}{l}\text { One thick un- } \\
\text { oriented mesh } \\
\text { of damaged } \\
\text { CNT-GO film. }\end{array}$ & $\begin{array}{l}\text { One sandwiched } \\
\text { CNT-GO film } \\
\text { with metallic } \\
\text { catalyst. }\end{array}$ & $\begin{array}{l}\text { One or multiple } \\
\text { sandwiched } \\
\text { chemical modified } \\
\text { self-terminating } \\
\text { CNT-GO film. }\end{array}$ & $\begin{array}{c}\text { One or multiple } \\
\text { sandwiched pristine } \\
\text { self-terminating CNT- } \\
\text { GO film. }\end{array}$ \\
\hline
\end{tabular}

Then, Table 11 compared the optical transparency and sheet resistance of between different films. The reported film was 130 times more conductive and $14 \%$ more transparent than the next best reported hybrid thin film. 


\begin{tabular}{ccc}
\hline Film & Transparency & sheet resistance \\
& @ $550 \mathrm{~nm}(\%)$ & $(\mathrm{k} \Omega / \square)$ \\
\hline Single layer of CNT & 98.1 & $>2 \mathrm{e} 6$ \\
\hline Single layer of rGO & 98.0 & $1.005 \mathrm{e} 3$ \\
\hline $\mathrm{CNT} / \mathrm{rGO}$ by sonication and heating ${ }^{275}$ & 86 & $2.4 \mathrm{e}-1$ \\
\hline $\mathrm{CNT} / \mathrm{rGO}$ by chemical modification ${ }^{270}$ & 81 & 8 \\
\hline Presented $\mathrm{rGO}+\mathrm{CNT}+\mathrm{rGO}$ layer by facile reflux & 94.72 & $6 \mathrm{e}-2$
\end{tabular}

It was crucial to tailor the hybrid film properties by controlling their composition and architecture at the nano-scale ${ }^{268}$; this method and the resulting novel hybrid structure was highly-tuneable even in the nano-meter scale and enables novel and promising approaches for energy storage, solar cells, semiconductors and biosensors. Mono-layers of GO could be formed on CNT thin films using this method. In addition only little energy was consumed due to low temperature processing.

\subsection{CONCLUSION}

This chapter reported a controllable and effective method to integrate 1-D CNT with the 2-D GO to obtain pristine CNT-GO hybrid structure which exhibited low electrical resistance $(60 \Omega /$ square $)$ at high optical transparency $(94.7 \%)$. The reported method also enables preferential deposition of large GO and control over the extent of wrinkling of the GO sheets. This reported method produced highly wrinkled surfaces $(20 \%)$ on the substrate that had not been reproduced or reported elsewhere. The hybrid film formation mechanism was due to the mild deoxygenation of facile oxygen functionalities on the soft GO matter; taking place more readily on large GO sheets with large basal planes, in agreement with Lerf-Klinowski's GO model. 


\section{CHAPTER 6: REDUCED GRAPHENE OXIDE GROWTH AND ITS MECHANISM}

This chapter reported the novel growth of reduced graphene oxide and its mechanism. This reported novel ability to obtain a complete substrate of rGO drastically reduced the both electrical resistance and electrical resistance homogeneity thus enabling its use in practical electronics and biosensors.

\subsection{INTRODUCTION}

Graphene is a single atomic layer of $\mathrm{sp}^{2}$ hybridized carbon atoms arranged in a honey-comb structure. Since the exfoliation of graphite into individual graphene sheets in 2004, ${ }^{1}$ its large carrier mobility, extraordinary thermal, chemical and optical properties had been highlighted. ${ }^{2}$ However the semi-metal and zero-band gap electronic structure of pristine graphene limited its use in electronic, sensing and optical applications. Some approaches to open the bandgap included post-processing of graphene such as strain engineering, ${ }^{3}$ lateral confinement, ${ }^{4}$ breaking inversion symmetry in bi-layer graphene, ${ }^{5}$ oxidation ${ }^{6}$ and usage of reduced graphene oxide (rGO). ${ }^{7,8}$

One method of obtaining a band gap was through the use of rGO. RGO was an inexpensive, aqueous processable material with an industrial-scalable production route. An efficient and scalable method for production of rGO involved chemical oxidation of graphite followed by exfoliation and reduction. ${ }^{9}$ After reduction, the increased pockets of $\mathrm{sp}^{2}$ hybridised carbon structure provided its mechanical strength and electrical conductivity. ${ }^{7}$

Despite these advantages and a substantial progress in rGO research over the years, rGO on substrates with good homogeneity and conductivity for practical applications were still not reproducable. ${ }^{10}$ This was due to the incomplete, poor coverage and different sizes of rGO flakes on substrates; posing a challenge to 
working with rGO fabrication.

It had been reported that Carbon Nanotubes (CNT) could be grown in ethanol Chemical Vapor Deposition (CVD) furnace using metal catalysts. But recently, it had been discovered that CNT could also be grown without a metal catalyst. A convincing model was still lacking on how carbon was nucleated and grown from oxides. Carbon growth had been reported using oxide seeds, such as nanodiamond particles, ${ }^{11} \mathrm{ZrO}^{12}$ and $\mathrm{SiO}_{\mathrm{x}}{ }^{13}$ via vapor-solid-solid (VSS) growth mechanisms. Attempts had been made to grow Graphene on substrates directly without any catalyst ${ }^{14}$ using plasma or high pressure. Attempts had also been made to repair Graphene and GO using ethanol $\mathrm{CVD}^{15,16}$ but no growth was observed. GO template carbonization is also noted under hydrothermal conditions $^{285}$.

Metals were commonly used as catalysts in several types of chemical reactions such as carbon nano-tube growth ${ }^{17}$ and Haber-Weiss reaction. ${ }^{18}$ One of the main properties of these metals was the presence of a free electron cloud on the surface of the metal which allowed for ready exchange between the metal surface and the electron acceptor molecules. This was the principle behind catalytic reactions. The metal acted as electron donor or in some cases as an electron acceptor. From graphene it was known that it provided 2D electron gas properties, comparable with metal surfaces ${ }^{19}$ and could be able to act similarly as catalyst.

From existing literature it was known that carbon cannot crystallize well by deposition from carburizing gases at low temperature without catalytic activation because of its strong $\mathrm{C}-\mathrm{C}$ bonds and high melting temperature. To form good crystalline carbon, the carbon atoms must dissolve, diffuse through metal particles, and crystallize on an appropriate facet that could act as a template to help the epitaxial growth of carbon crystals. ${ }^{20}$ Thus it was hypothesized that rGO on the substrate could act similar to the described metal particles, and help the growth of carbon crystals/layers. In this chapter, this hypothesis and the growth of rGO, which not only extend size but also homogeneity of its electrical conductivity, enabling rGO use in electronic devices by the extended growth of GO flakes using ethanol CVD was tested 
and reported.

\subsection{GROWTH OF RGO}

\subsubsection{GO SYNTHESIS}

To grow and extend the size of rGO, a modified Hummers method ${ }^{39,} 91$ (obtained in earlier chapters) was first used to obtain Graphene Oxide (GO) sheets. Briefly, graphite flakes were pre-oxidized using concentrated $\mathrm{H}_{2} \mathrm{SO}_{4}$, ultra-sonicated and filtered to remove small graphite debris. Then, the dried pre-oxidized graphite powder was added into concentrated $\mathrm{H}_{2} \mathrm{SO}_{4}$ and $\mathrm{KMnO}_{4}$. Thereafter, the GO was electrostatically attached to the APTES-functionalized $\mathrm{SiO}_{2}$ substrates.

\subsubsection{TRANSFER OF GO TO SUBSTRATE}

$\mathrm{SiO}_{2}$ substrates were RCA cleaned two times, dried and then rinsed in ethanol before being placed into $1 \% \mathrm{v} / \mathrm{v}$ 3-APTES ethanol solution for 1 hour. The 3APTES $\mathrm{SiO}_{2}$ substrates were then placed in oven for 1 hour to complete the functionalization process. After the substrates cooled, prepared GO solution was drop-casted and allowed to stand for 1 hour. Thereafter, the substrates were rinsed, blow-dried and kept ready for further treatment.

\subsubsection{REDUCTION OF GO BY HYDRAZINE}

Prepared GO substrates were placed in a glass petri-dish with $400 \mu$ of hydrazine monohydrate dropped in the centre of the dish and then sealed. The petri dish was heated to $70^{\circ} \mathrm{C}$ and left overnight for complete reduction. Thereafter, the substrates were rinsed and blow-dried.

The substrates, with 1-2 layers of GO on top, were placed on a holder and placed in a CVD tube furnace setup (Figure 47). The furnace was purged with Ar for 30 minutes at $300 \mathrm{sccm}$ to remove $\mathrm{O}_{2}$ that can react with the GO to form $\mathrm{CO}$ and $\mathrm{CO}_{2}$. After purging, the flow of Ar was adjusted to $100 \mathrm{sccm}$ and $\mathrm{H}_{2}$ turned on to $20 \mathrm{sccm}$. Next, the valves to ethanol precursor were turned on and 
the furnace heated to $950^{\circ} \mathrm{C}$ at $40^{\circ} \mathrm{C}$ per minute. The gases were passed through an ethanol bubbler held at $0^{\circ} \mathrm{C}$ in a water bath chiller before entry into the furnace. The absolute ethanol in the bubbler was changed at the start of every experiment since humidity adsorbed decreases the vapour concentration ${ }^{286}$. The rate of ethanol consumed was $2.5 \mathrm{ml} /$ hour $\left(3.5 \times 10^{-4} \mathrm{ml}\right.$ per $\mathrm{sccm}$ gas flow). After 2 hours, the furnace was turned off and the tube left to cool slowly to room temperature while $\mathrm{H}_{2} / \mathrm{Ar}$ continued to flow.

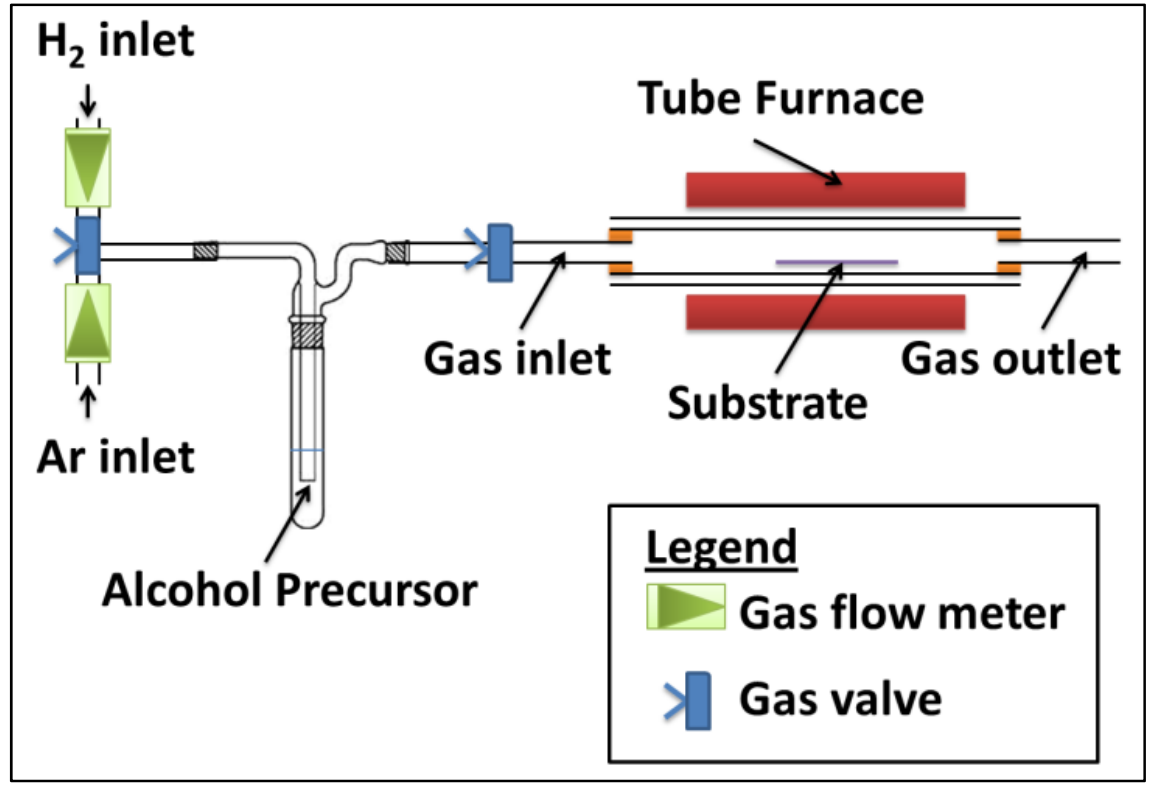

Figure 47: An atmospheric pressure CVD setup with alcohol vapors for growth of rGO 


\subsection{CHARACTERIZATION OF CVD RGO}

\subsubsection{SEM AND TEM CHARACTERIZATION}

A typical SEM image of GO flakes on $\mathrm{SiO}_{2}$ substrate was shown in Figure 48a. After a 30-minutes ethanol CVD treatment, the total rGO coverage increased to ca. 75\% (yellow highlighted area) shown in Figure 48b. Figure 48c showed a high-magnification SEM image on the new growths bridging between edges of two rGO flakes.

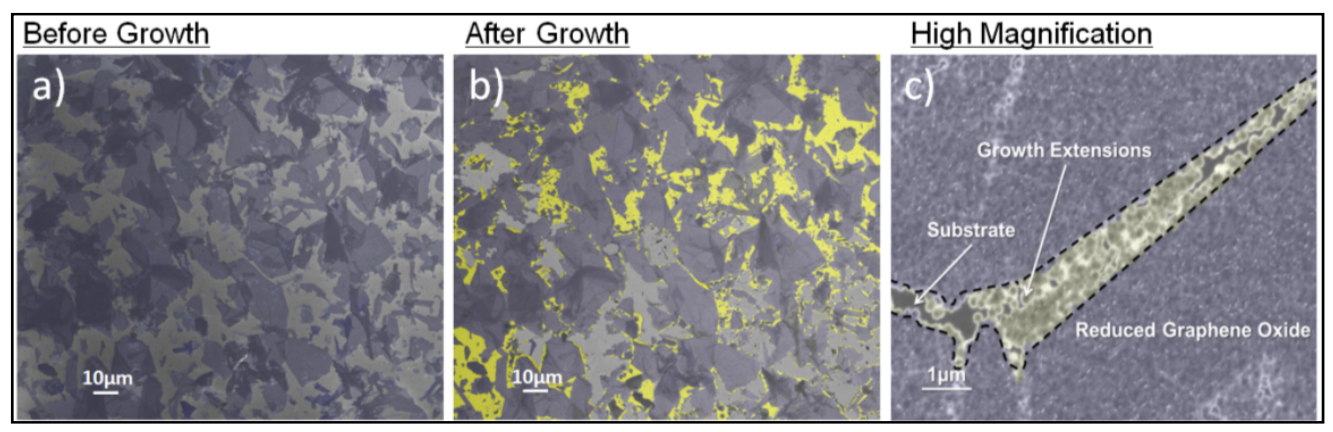

Figure 48: SEM image of a) before ethanol CVD treatment GO flakes [highlighted in purple] on $\mathrm{SiO}_{2}$ substrate with ca. $60 \%$ coverage b) after a 30-minutes CVD treatment RGO flakes [highlighted purple] and the extended rGO growths [highlighted in yellow] with a total ca. $75 \%$ coverage. c) High-magnification image on the new growths bridging between two rGO flakes.

A TEM image of the boundaries between the existing flake and new growth after 2 hours ethanol CVD treatment was shown in Figure 49. In the TEM image, the lighter contrast of the newly grown rGO was highlighted in yellow. The contrast difference was due to the difference in number of layers between the pre-existing rGO flake and newly grown rGO. It was observed that the new growth ends with circular edges, similar to observations in the highmagnification SEM image. 


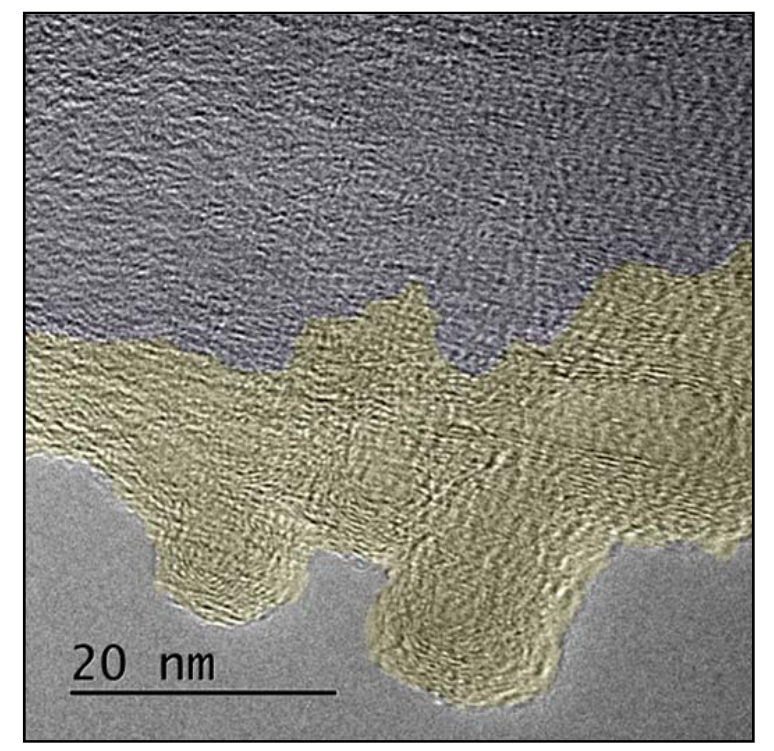

Figure 49: TEM image of pre-existing rGO (darker) and new grown rGO (highlighted in yellow)

To elucidate the effect of the GO, clean substrates and also APTESfunctionalized substrates were subjected to the same ethanol CVD process for up to 2 hours. These control substrates without initial GO flakes, showed neither carbon deposit nor carbon spectrum on the SEM and Raman respectively. This showed that initial GO flakes were essential to enable further rGO growth in the ethanol CVD setup ${ }^{287}$. The initial rGO flakes acted as a crystal nucleus for further growth of these flakes.

Observation from the SEM picture of the same GO flakes before and after CVD treatment (Figure 50a and b) showed that the original GO were still present after the CVD treatment and there was no change in size. However, a different contrast between the original graphene flake and substrate was observed under the SEM. Also, the extended growths were observed to be lighter in shade compared to pre-existing flakes. The contrast mechanism was due to e-beaminduced surface potential between graphene of different band gaps ${ }^{288}$. Differences in the number of rGO layers were also observed as different contrast in the picture, the lightest being single or few layers and darker indicating more layers ${ }^{5}$. Thus using the SEM allowed effective and efficient identification of the existing flake, the new growth area and the relative 
thickness of the rGO. Within 2 hours, the substrate of $c a .1 \mathrm{cmx} 1 \mathrm{~cm}$ was completely covered with rGO.

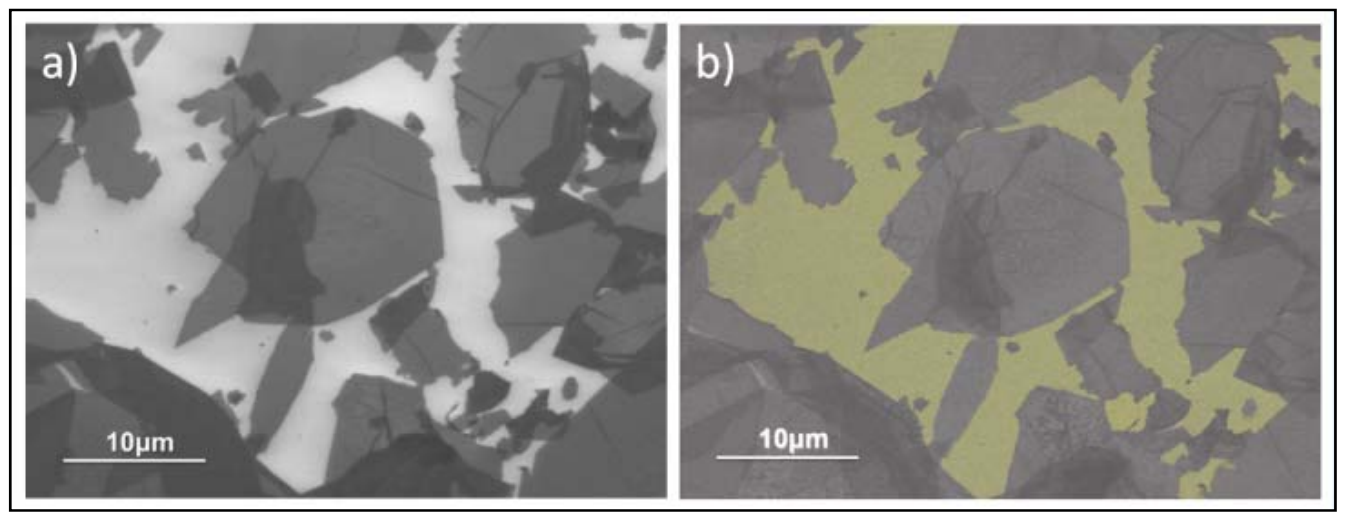

Figure 50: SEM image of a) GO flakes before and b) rGO flakes and extended rGO growths [highlighted in yellow] after 2 hours ethanol CVD treatment. The size and features of the GO were preserved after the CVD treatment

After a 2 hour ethanol CVD treatment, the entire substrate was completely covered with the rGO flakes and rGO new growth. Figure 51a showed the substrate before growth and Figure $51 \mathrm{~b}$ showed the substrate after growth.

To further elucidate the growth process, a higher magnification image was taken after a 30 minute ethanol CVD treatment (Figure 51c). At this stage, the substrate was only partially covered with the newly grown rGO flakes. Carbon islets were observed to deposit preferentially at the edges of template flakes. Clean substrates without GO were also ethanol CVD treated up to 2 hours and no growth was observed. Thus GO acted as a catalyst for preferential growth sites for rGO new growths. The $2 \mathrm{D}$ electron gas properties of $\mathrm{GO}^{245,289}$ which was similar to that of metal surfaces helped to catalyse the rGO new growths in the ethanol CVD treatment. 


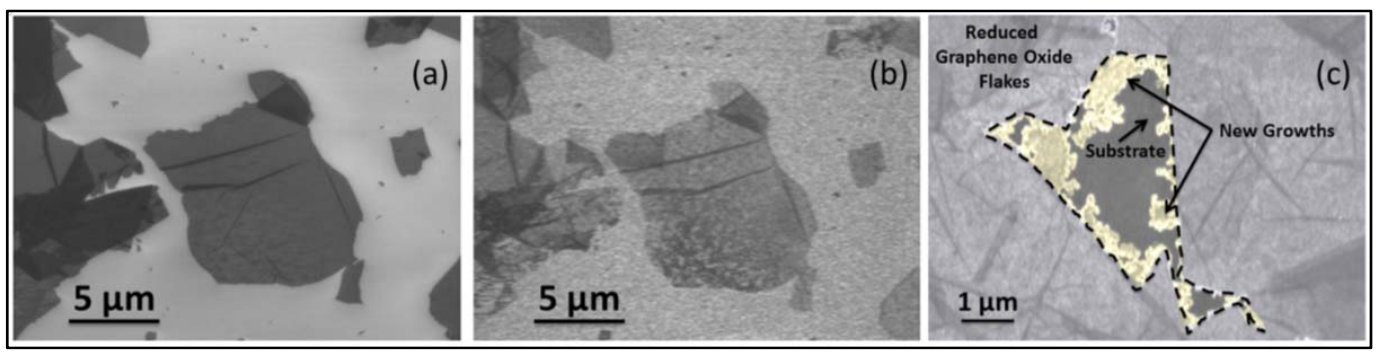

Figure 51: SEM images of (a) before ethanol CVD treatment GO flake templates on SiO2 substrate (b) after a 2 hour CVD treatment rGO flake with rGO new growths completely covering substrate (c) High-magnification image of the new grown flakes bridging a gap between rGO flakes.

Figure 52a showed the electron microscope image of free-standing reduced GO after 2 hours of CVD treatment. Figure 52b showed the selected area electron diffraction (SAED) of the same sample, and the patterns matched those expected of few layered graphene-based sheets ${ }^{290,291}$. The well-defined diffraction spots in SAED also indicated that the structure of the reduced GO had an internal ordered crystalline structure after the CVD treatment. However, after a minute of exposure to the electron beam during the TEM observation, the reduced GO was vaporized, which was possibly caused by the evaporation of the hydrogen and oxygen-containing functional groups still existing on the reduced GO.
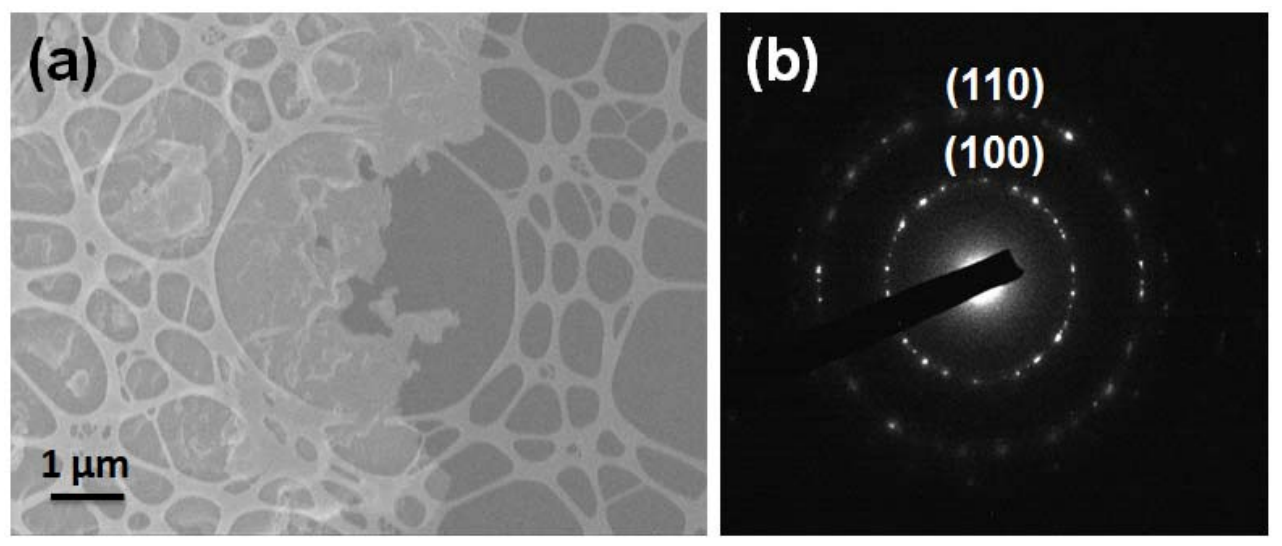

Figure 52: Electron microscope image of 2-hour treated (a) GO on lacey carbon (b) SAED patterns of reduced GO at the newly formed GO. 


\subsubsection{RAMAN CHARACTERIZATION OF RGO}

Raman spectroscopy was important for the characterization of the graphene structure, thickness and defects. The optical micrograph of rGO flake and the substrate was shown in Figure 53a. Figure 53b showed Raman spectrum on the rGO flakes processed at different CVD durations. Both Graphitic-band (Gband, $\sim 1575 \mathrm{~cm}^{-1}$ ) and second-order Defect-band (2D-band, $\sim 2700 \mathrm{~cm}^{-1}$ ) were observed in Highly Ordered Pyrolytic Graphene (HOPG), GO and rGO in agreement with the literature. ${ }^{23}$ The G-band of unprocessed GO $\left(1599 \mathrm{~cm}^{-1}\right)$ was up-shifted compared with that of HOPG $\left(1575 \mathrm{~cm}^{-1}\right)$ and attributed to the presence of isolated double bonds that resonated at a higher frequency in GO than graphite. ${ }^{24}$ The G-band of the 2 hour CVD processed sample $\left(1585 \mathrm{~cm}^{-1}\right)$ down-shifted closer to the G-band of HOPG. Furthermore, the 2D-band upshifting of CVD samples towards the graphite 2-D band position also suggested more significant structural changes towards graphite as CVD process time increased. $^{25}$

The observation of the D-band of GO and $\mathrm{rGO}$ at $\sim 1350 \mathrm{~cm}^{-1}$ suggested the presence of disordered structural defects from the attachment of hydroxyl and epoxide groups on the carbon plane. ${ }^{23}$ The Defect-band/Graphitic-band (D/G) intensity ratio was observed to decrease from 1.02 in GO samples to 0.88 in the 2 hour CVD samples. Decrease in the D/G intensity value corresponded to an increase in average size of $\mathrm{sp}^{2}$ domains. ${ }^{26}$ Thus, ethanol CVD method increased the average size of $\mathrm{sp}^{2}$ domains on existing GO flakes. Using the TuinstraKoenig $^{27}$ empirical method, the lateral domain size of the $\mathrm{sp}^{2}$ graphitic ring cluster on the GO flake after 2 hours of Ethanol CVD treatment was calculated to be $\sim 4.8 \mathrm{~nm}$. The treatment by ethanol CVD increased the spatial dimension of the $\mathrm{sp}^{2}$ region of existing $\mathrm{rGO}$ flake thus resulting in an increase in conductivity. However, it was observed that this graphitization was not the only reason that led to the increase in electrical conductivity.

Raman spectroscopy was also performed on the spaces between rGO flakes for all samples as shown in Figure 53c. The contrast difference between the initial rGO flakes and the substrate was used as a guide to perform Raman. Measurement of spaces from the 1 hour and 2 hours samples showed peaks of 
$1351 \mathrm{~cm}^{-1}, 1585 \mathrm{~cm}^{-1}, 2711 \mathrm{~cm}^{-1}$ which were consistent with the $\mathrm{D}, \mathrm{G}$ and $2 \mathrm{D}$ bands of typical rGO. Whereas, no carbon Raman signals were obtained for the 15 minutes and 30 minutes CVD processed chips, consistent with SEM observation that $\mathrm{rGO}$ growth starts from the edges and did not fill up the large spaces in the 15 and 30 minutes samples. The Raman G-band carbon intensity of the 1 hour sample and GO flake was $\sim 70 \%$ and $\sim 40 \%$ of the 2 hour sample's intensity respectively. Since Raman intensity of the allowed peak was proportional to the volume of sample, ${ }^{28}$ the intensity difference suggested a smaller amount of new rGO in the 1 hour versus the 2 hour sample.

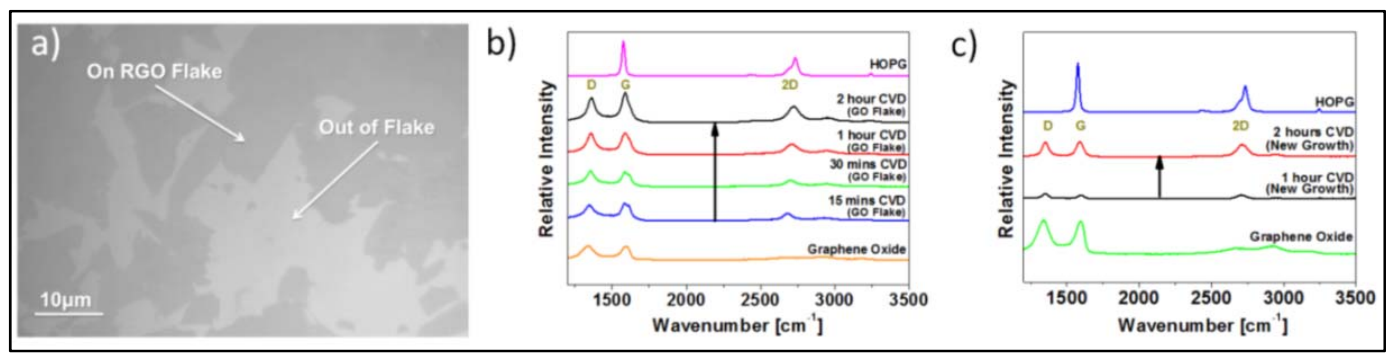

Figure 53: a) Optical micrograph [100x magnifications] of rGO flakes treated with 2hours of ethanol CVD, showing contrast difference between rGO flake and out of flake areas. Raman spectra $[488 \mathrm{~nm}]$ on b) rGO flake and c) out of flake areas, with different CVD processing times, in comparison to GO and Highly Ordered Pyrolytic Graphite (HOPG).

The G-band and the 2-D band of the rGO growths were also observed to down and up-shift respectively as CVD time increased. Both bands shifted towards that of HOPG, an indication of structural changed towards graphite as process time increased. ${ }^{21}$ The $\mathrm{D} / \mathrm{G}$ peak intensity ratio of the rGO growth in the 2 hours CVD sample decreased to 0.71 from GO's ratio of 1.02, suggested a significant increase in the average size of the $\mathrm{sp}^{2}$ domain. This new rGO growth's $\mathrm{D} / \mathrm{G}$ intensity ratio of 0.71 was remarkably low compared to 0.88 on rGO flakes after 2 hours ethanol CVD or 1.56 on rGO flakes reduced with 12 hours of hydrazine vapor. ${ }^{17}$ Using the Tuinstra-Koenig empirical formula, ${ }^{27}$ the lateral domain size of the $\mathrm{sp}^{2}$ graphitic ring cluster on the newly grown $\mathrm{rGO}$ was found 
to be $\sim 5.7 \mathrm{~nm}$.

In the 2D-band of HOPG and in the graphene sheets of 5 or more layers, there were 2 components, $2 \mathrm{D}_{1}\left(2690 \mathrm{~cm}^{-1}\right)$ and $2 \mathrm{D}_{2}\left(2732 \mathrm{~cm}^{-1}\right)$ as reported earlier. ${ }^{23}$ Figure 54 showed this deconvoluted $2 \mathrm{D}_{1}$ and $2 \mathrm{D}_{2}$ bands of HOPG. It was noted that in the ethanol CVD treated chips, both new growth and pre-existing GO flakes, did not show the $2 \mathrm{D}_{1}$ and $2 \mathrm{D}_{2}$ bands typical of HOPG, thus suggesting the thickness of the layers must be less than 5 layers, which was confirmed with the Atomic Force Microscopy (AFM) reported later in this chapter.

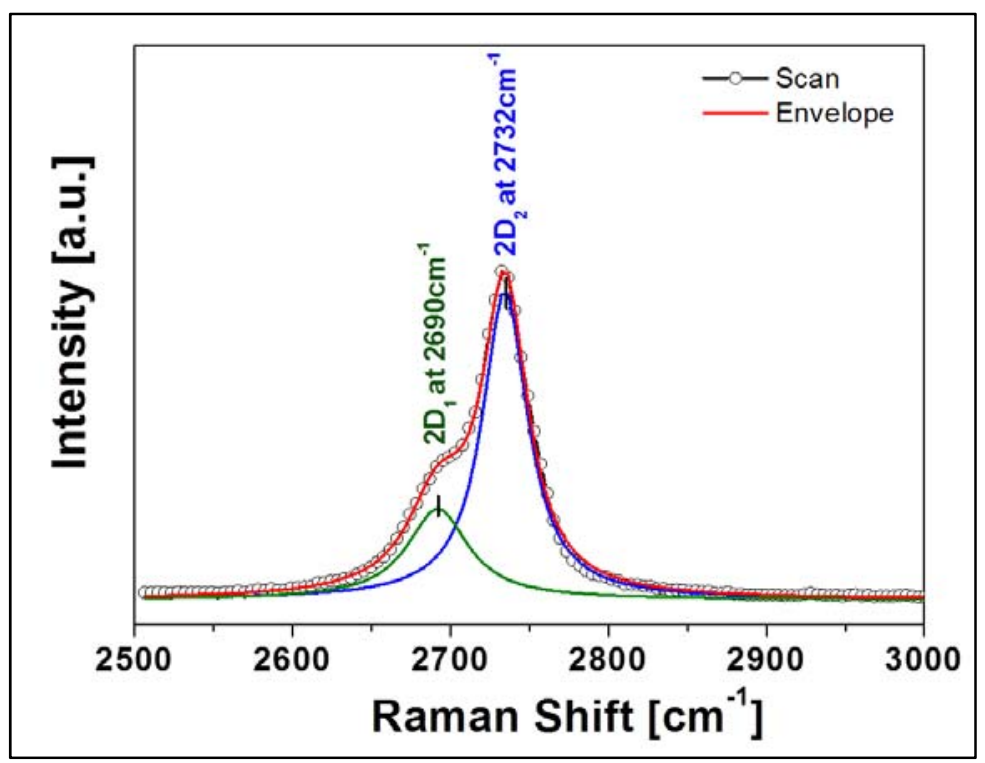

Figure 54: Raman spectra of 2D band of HOPG with its 2D1 and 2D2 components deconvoluted.

An optical image of a 2-hour ethanol CVD processed chip was shown in Figure 55a. Raman spectroscopy could distinguish the number of layers of carbon. Increasing the number of layers up-shifts the Raman 2D peak position ${ }^{245}$. A Raman image was then reconstructed by mapping out the peak position in the samples. This reconstruction was shown in Figure 55b. The peak position shift of $23 \mathrm{~cm}^{-1}$ between the 2D peaks was shown in Figure $55 \mathrm{c}$. From the Raman map, the overall coverage of the substrate by pre-existing rGO and newly grown rGO was ca. $100 \%$. 


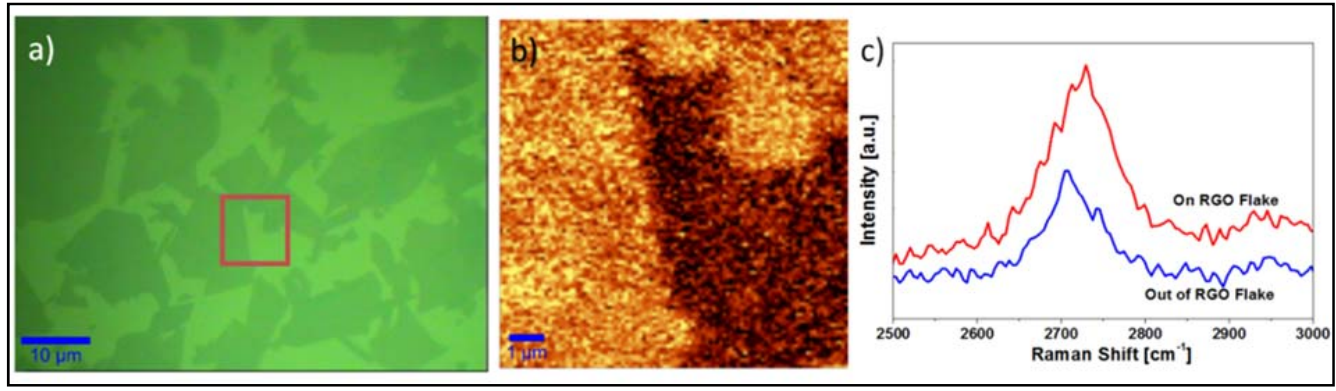

Figure 55: (a) Optical microscope image of 2-hour processed reduced GO flakes on $\mathrm{SiO}_{2}$ substrate (b) Raman mapping at edges of rGO flakes showing $100 \%$ carbon coverage. (c) Raman spectrum of both on and out of rGO flakes.

\subsubsection{XPS AND AFM CHARACTERIZATION OF CVD RGO}

Figure 56 reported the C1s XPS spectra of original GO, 15 mins CVDprocessed rGO and 120 mins CVD-processed rGO. In Figure 56a, the C1s signal was deconvoluted into $\mathrm{C}-\mathrm{C}(284.6 \mathrm{eV}), \mathrm{C}-\mathrm{OH}(286.3 \mathrm{eV})$ and $\mathrm{C}=\mathrm{O}$ (287.2). These assignments were in agreement to previous reports. ${ }^{10,25}$ After CVD treatment of 15 minutes (Figure 56b), the carbonyl and epoxy acid signatures were diminished from their starting intensities and were dwarfed by the $\mathrm{C}-\mathrm{C}$ signals. The atomic percentage (at \%) for the different functional groups were calculated with respect to the total area of the $\mathrm{C} 1 \mathrm{~s}$ peak. $\mathrm{C}-\mathrm{C}$ bond accounted for 49.6, 76.5 and 84.2 at $\%$ in the rGO sample after CVD treatment of 0,15 and 120 mins respectively. The $\mathrm{C}=\mathrm{O}$ bond peak (initially at 9.7 at $\%$ ) was completely removed after 15 mins of CVD treatment and could possibly be reduced to $\mathrm{C}-\mathrm{O}$ bonds, a reduced species arising from reduction of the $\mathrm{C}=\mathrm{O}$ in GO. Between the 15 and 120 mins sample the C-O at\% decreased from 23.5 to $15.8 \mathrm{at} \%$, suggesting that graphitization and growth had taken place. The XPS results confirmed the identity of reduced $\mathrm{GO}$, complete removal of $\mathrm{C}=\mathrm{O}$ bonds and that graphitization and growth could continue up to 120 mins as shown in Figure 56c. 

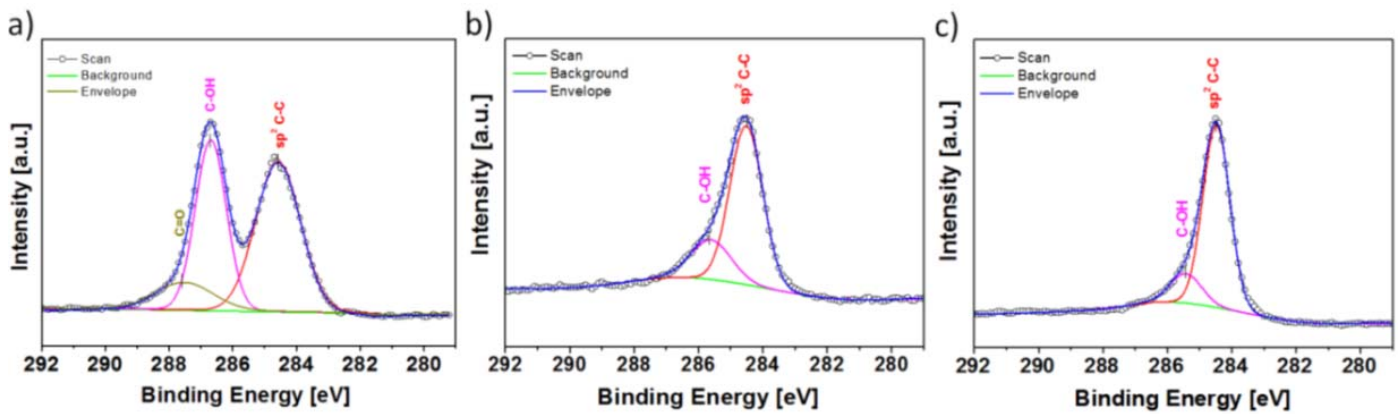

Figure 56: XPS spectra and identified binding energies for a) GO and b) rGO after 15 mins and c) 120 mins of ethanol CVD treatment

To elucidate more on the different growing conditions of the rGO, the ethanol and the hydrogen gas input were varied. The $\mathrm{C} 1 \mathrm{~s}$ spectral of $\mathrm{rGO}$ shown in Figure 57 could be deconvoluted using Lorentzian with Gaussian broadening curve-fitting into $\mathrm{C}=\mathrm{O}(\sim 287.2 \mathrm{eV}), \mathrm{C}-\mathrm{OH}(\sim 286.3 \mathrm{eV})$ and $\mathrm{C}-\mathrm{C}(284.5 \mathrm{eV})$. These values were in agreement with previous reports ${ }^{5,13,106}$. The C-C spectra positions for all 5 samples were detected to be at $284.5 \mathrm{eV}$. However, there were variations in $\mathrm{C}=\mathrm{O}$ and $\mathrm{C}-\mathrm{OH}$ spectra positions for different samples. The sample treated with more hydrogen gas had both $\mathrm{C}=\mathrm{O}$ and $\mathrm{C}-\mathrm{OH}$ down-shifted, followed by sample with standard CVD treatment, CVD treatment with no ethanol, hydrazine treatment and GO. The CVD treatment with more hydrogen had chemical shifts more towards that of HOPG which indicated that the bonds present in the compound was more similar to that of HOPG. This showed that the presence of hydrogen gas in the ethanol system acted as an etchant which could anisotropically etch amorphous carbon to produce reduced GO closer to that of HOPG, similar to what had been reported for graphene systems ${ }^{292}$. Hydrogen also preferentially etch away stacked carbon $(16 \mathrm{~kJ} / \mathrm{mol})$ and some oxygen moieties due to the weaker bond compared to in-plane carbon (345 $\mathrm{J} / \mathrm{mol}$ ) thus leading to more graphitized rGO instead of amorphous carbon ${ }^{293}$. The atomic percentages of the bonds present in the XPS spectra was then calculated and presented in Table 12. 


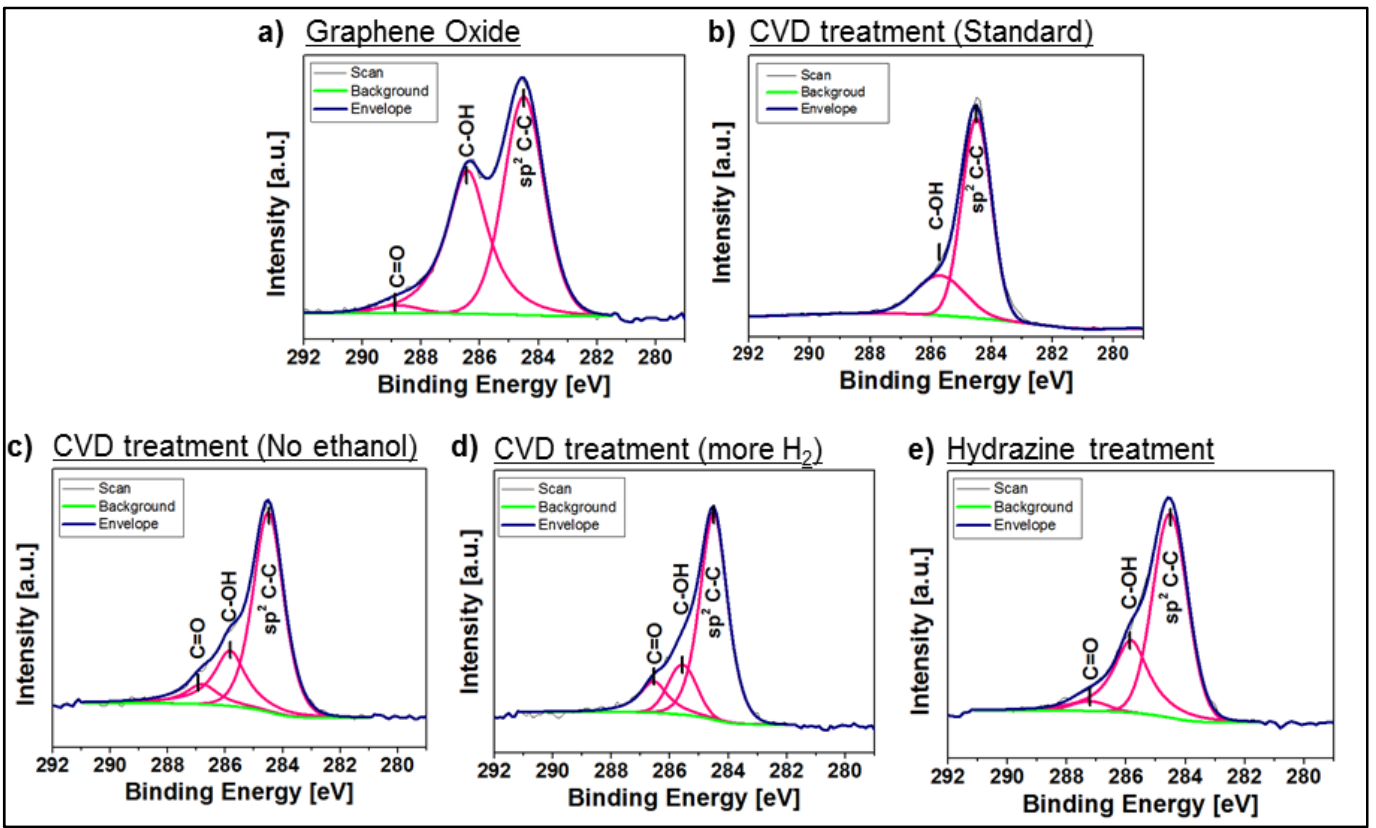

Figure 57: C1s XPS spectra (hv=1486.6eV) collected on (a) GO; and Reduced GO reduced in similar CVD setup with (b) 1 hour standard CVD treatment (c) 15 minutes CVD treatment with no ethanol (d) 1 hour CVD treatment with more $\mathrm{H}_{2}$ gas (e) 12-hour hydrazine reduced sample. The different components related to various chemical shifts of carbon bonds were indicated. 
Table 12: Atomic percentage of different carbon bonds identified by XPS on monolayered GO and rGO reduced under different conditions

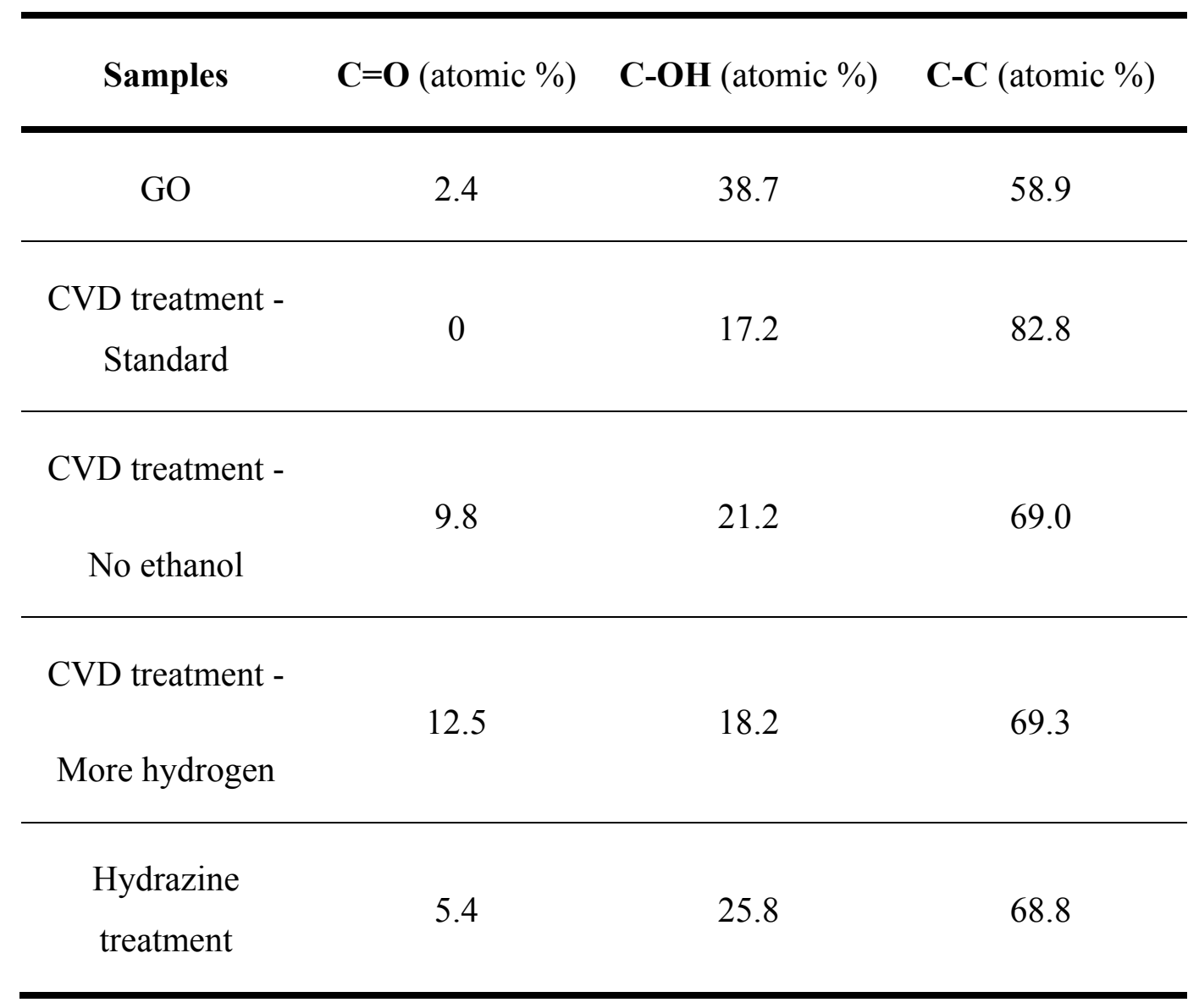

In all the rGO samples, the atomic percentage of $\mathrm{C}-\mathrm{OH}$ bonds decreased while the percentage of $\mathrm{C}-\mathrm{C}$ bonds increased. For the sample with standard CVD treatment, the $\mathrm{C}=\mathrm{O}$ bond peak (initially at $2.4 \%$ ) was completely removed indicating an efficient graphitization. The $\mathrm{C}=\mathrm{O}$ bond in the $\mathrm{CVD}$ treatment had been reduced to $\mathrm{C}-\mathrm{O}$ bonds, a reduced species of $\mathrm{C}=\mathrm{O}^{5}$. However, in other reduced samples, the atomic percentage of $\mathrm{C}=\mathrm{O}$ bonds increased. This was not in agreement with existing literature ${ }^{106,245,267}$; this was due to the initial large GO flakes used. With larger GO flakes, the atomic percentages of both $\mathrm{C}=\mathrm{O}$ and $\mathrm{C}-\mathrm{OH}$, which were predominantly found at the edges of the flakes, were much lower in the reported GO compared to the GO synthesized in existing literature ${ }^{13,96,256}$.

The remaining $\mathrm{C}=\mathrm{O}$ bonds and higher atomic percentage of the $\mathrm{C}-\mathrm{OH}$ bonds indicated that the presence of ethanol, and the right flow rate of hydrogen gas, 
were critical in the complete and efficient graphitization of rGO. The CVD procedures, gas flow parameters and ethanol setup used in this study had been optimized for the growth of carbon nanotubes cleanly without amorphous carbon. Deviations from the optimal condition resulted in residual $\mathrm{C}=\mathrm{O}$ bonds. Using CVD treatment of no ethanol, more hydrogen or using hydrazine treatment resulted in $\mathrm{rGO}$ products with similar amount of $\mathrm{C}-\mathrm{C}$ atomic percentage $(\sim 69 \%)$, but with varying $\mathrm{C}-\mathrm{OH}$ and $\mathrm{C}=\mathrm{O}$ depending on condition used. With 1 hour CVD treatment, the $\mathrm{C}-\mathrm{C}$ atomic percentage increased to $82.8 \%$. As a result, tailoring of rGO products with specific bonds was realized by modification of reaction conditions.

In addition to $\mathrm{C} 1 \mathrm{~s}$ spectra, the $\mathrm{C}$ KLL spectra were also measured to compare $\mathrm{sp}^{2}$ to $\mathrm{sp}^{3}$ ratio in the treated samples. Figure 58 showed the spectra of the first derivative of X-ray excited C KLL between diamond, HOPG and CVD treated samples (treatment times $=15,30,60,120$ minutes). The $\mathrm{D}$ value between the maximum of the positive-going excursion and the minimum of the negativegoing excursion in the derivative XAES spectra represented the energy separation. This D value conformed to linear proportionality between the graphite and diamond. ${ }^{30}$ Thus increasing D was associated to the increasing number of $\pi$ electrons and the percentage of $\mathrm{sp}^{2}$ area in the film. ${ }^{31,32}$ The assignment of peaks for D value calculations were as reported in previous literature. $^{33}$ 


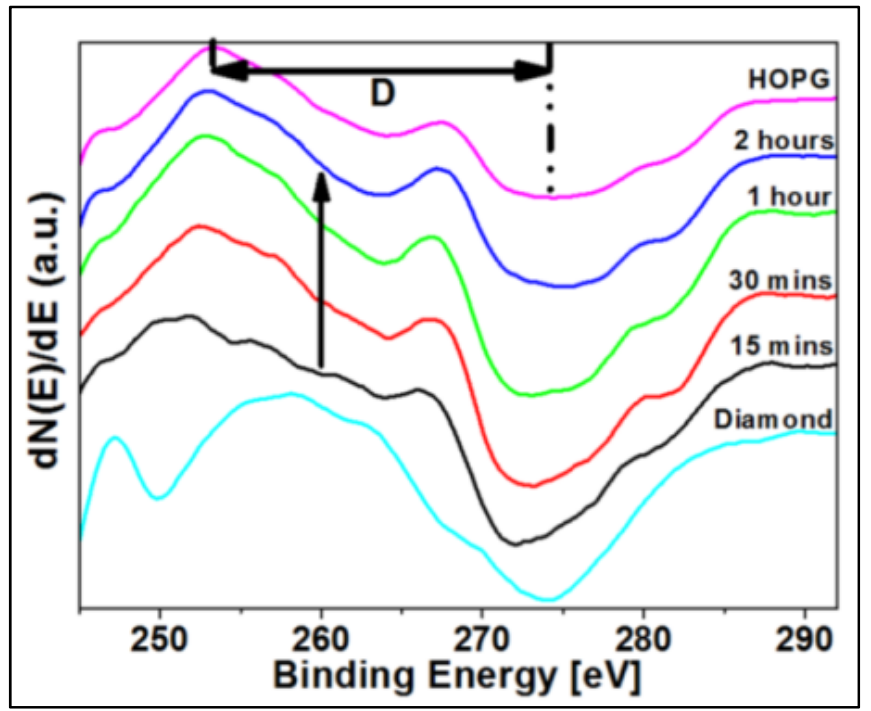

Figure 58: First derivative of the X-ray excited C KLL spectra of HOPG, CVD treated samples and diamond. The $D$ value which corresponded to the area ratio of $\mathrm{sp}^{2}$ increases with increasing ethanol CVD process time.

Table 13: D parameter and $\mathrm{sp}^{2}$ percentage area of different carbon samples

\begin{tabular}{ccc}
\hline Samples & D (eV) & \% sp $\mathbf{~}^{2}$ \\
\hline HOPG & 21.87 & 100 \\
\hline rGO (2 hours CVD) & 21.45 & 93 \\
\hline rGO (1 hour CVD) & 20.00 & 77 \\
\hline rGO (30 mins CVD) & 19.79 & 66 \\
\hline rGO (15 mins CVD) & 19.58 & 63 \\
\hline rGO (12 hours hydrazine vapor) & 18.74 & 50 \\
\hline GO & 18.23 & 42 \\
\hline
\end{tabular}


The $\mathrm{D}$ values and calculated $\mathrm{sp}^{2}$ percentage through linear interpolation between HOPG and diamond were tabulated in Table 13. With increasing treatment time, the percentage of $\mathrm{sp}^{2}$ increased, due to the contributions from graphitization of existing flakes and new enlarged growth of the rGO. The calculated $42 \% \mathrm{sp}^{2}$ area in as-synthesized GO was in good agreement with literature. ${ }^{7}$ The percentages of $\mathrm{sp}^{2}$ sites on $\mathrm{rGO}$ increased to $93 \%$ with 2 hours of ethanol CVD treatment. This $\mathrm{sp}^{2}$ sites increase corresponded to a factor of more than 2 times from the initial GO flake.

AFM images with RMS roughness value and corresponding height profile of rGO flake at different ethanol CVD treatment time were shown in Figure 59. In the first 30 minutes of ethanol CVD treatment, the thickness of GO flake increased to $\sim 2 \mathrm{~nm}$; after which carbon islets of $\sim 2 \mathrm{~nm}$ started to form on the GO flake and increased in distribution. These islets on the flake did not agglomerate into a continuous layer. This was reflected in the RMS roughness of the rGO flake which increased significantly from 0.9 (30 mins ethanol CVD treatment) to 3.1 (2 hour ethanol CVD treatment). This 2-4nm height corresponded to 2-4 layer of GO flakes ${ }^{34}$ and was in agreement with the Raman observation of less than 5 rGO layers.

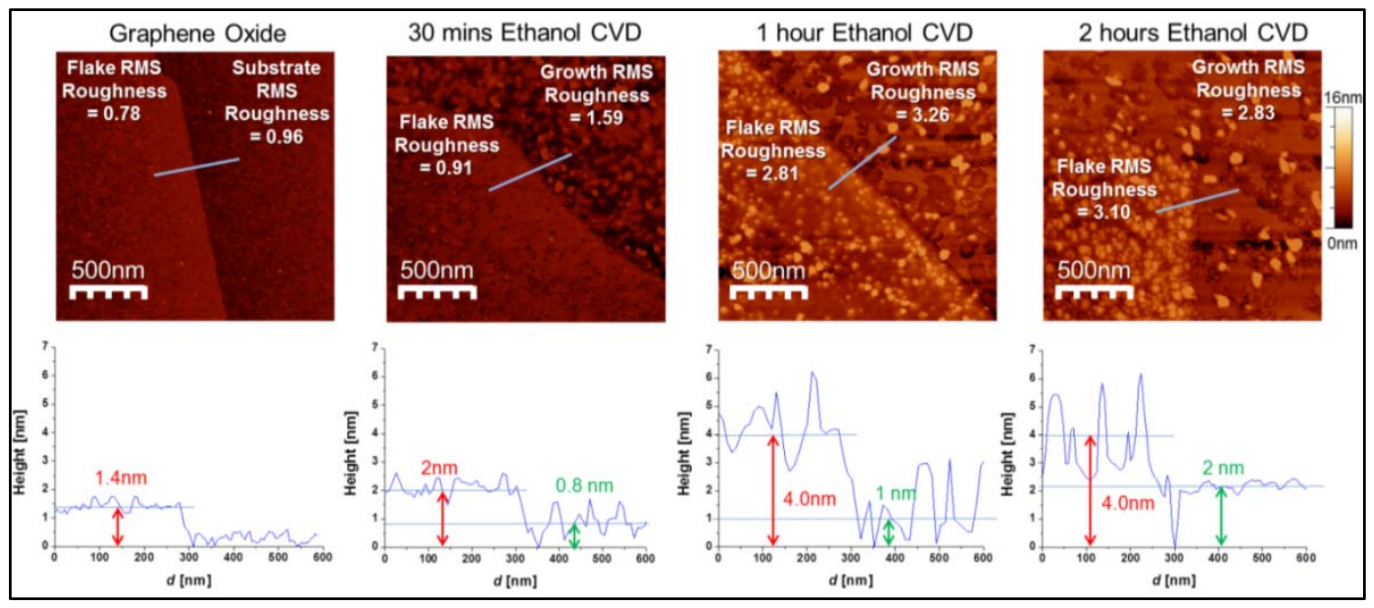

Figure 59: AFM images with Root Mean Squared (RMS) roughness value and corresponding height profile of GO with different stages of CVD process. 
After 30 minutes of ethanol CVD treatment, carbon islets of diameter $<50 \mathrm{~nm}$ were observed outside the rGO flake. In contrast, to the islets formed on rGO flakes, the islets on the substrate agglomerated as time progressed, to larger islets of $\sim 80 \mathrm{~nm}$ in the 1 hour sample and finally into a layer up to $400 \mathrm{~nm}$ across in the 2 hour sample. This agglomeration corresponded to a peak in RMS value at 3.3 (1 hour CVD) which then decreased to 2.8 (2 hours CVD).

\subsubsection{Electronic Properties of CVD RGO}

Table 14: Electrical measurements of rGO processed under different conditions

\begin{tabular}{|c|c|c|c|c|c|}
\hline \multirow{2}{*}{$\begin{array}{l}\text { Reduction } \\
\text { Conditions }\end{array}$} & \multirow{2}{*}{$\begin{array}{c}\text { Hydrazine } \\
\text { Vapor } \\
\text { Reduction }\end{array}$} & \multicolumn{4}{|c|}{ Ethanol CVD Reduction } \\
\hline & & $15 \mathrm{mins}$ & 30 mins & 1 hour & 2 hours \\
\hline $\begin{array}{c}\text { Average } \\
\text { Resistance }(\Omega / \square)\end{array}$ & $1.99 \mathrm{E}+06$ & $6.10 \mathrm{E}+04$ & $2.87 \mathrm{E}+04$ & $\begin{array}{c}2.23 E+0 \\
4\end{array}$ & $4.68 \mathrm{E}+03$ \\
\hline $\begin{array}{c}\text { Absolute Standard } \\
\text { Deviation ( } \mathbf{( \Omega )}\end{array}$ & $1.60 \mathrm{E}+06$ & $4.42 \mathrm{E}+04$ & $1.25 \mathrm{E}+04$ & $\begin{array}{c}7.14 \mathrm{E}+0 \\
3\end{array}$ & $7.70 \mathrm{E}+02$ \\
\hline $\begin{array}{c}\text { Relative Standard } \\
\text { Deviation (\%) }\end{array}$ & 805 & 72.6 & 43.6 & 32.1 & 16. \\
\hline
\end{tabular}

The effect of CVD process time on the electrical resistance on the samples using a 4-point probe setup was also investigated. Samples were reduced differently with 15, 30, 60 and 120 minutes of ethanol CVD and hydrazine vapor. 5 samples were prepared for each condition. Their electrical resistance and standard deviation were then measured, as shown in Table 14. For accurate comparison, all the chips were made in one single batch. The average resistance of samples after 15 minutes of ethanol CVD was 2 magnitudes less than hydrazine reduced samples. This was due to the improved graphitization ${ }^{40}$ of 
the rGO as shown in the Raman and XPS analysis. However, the relative standard deviation for these 15 mins treated sample only decreased marginally by $9.8 \%$. As CVD treatment time increased to 2 hours; the electrical resistance decreased by $99.8 \%$ and the relative standard deviation decreased by $79.5 \%$ compared to hydrazine vapor reduced samples. This resistance drop was attributed to improvement between inter-flake connections while the standard deviation drop was attributed to the complete $100 \%$ rGO coverage of sample chips, as show in SEM and Raman Mapping.

Back-gated Field-Effect Transistors (FET) based on 2-hour ethanol CVD processed rGO sheets was fabricated and their electrical properties tested under ambience temperature and pressure. The channel length of $100 \mu \mathrm{m}$ was chosen to ensure that the transport was bulk limited and the role of contacts minimized. Figure 60a illustrates a typical drain current $\left(\mathrm{I}_{\mathrm{d}}\right)$ versus drain voltage $\left(\mathrm{V}_{\mathrm{d}}\right)$ curve prepared with ethanol CVD processed rGO field-effect transistor at six discreet gate voltages $\left(\mathrm{V}_{\mathrm{g}}\right)$. The linear output behaviour indicated good ohmic contact between the rGO film and electrodes. Figure $60 \mathrm{~b}$ illustrated the same transistor $I_{d}$ under sweeping $V_{g}$ at fixed $V_{d}=0.1 \mathrm{~V}$. The charge neutrality point was shifted to the positive side due to $\mathrm{p}$-doing from persistent moisture and oxygen. ${ }^{35}$
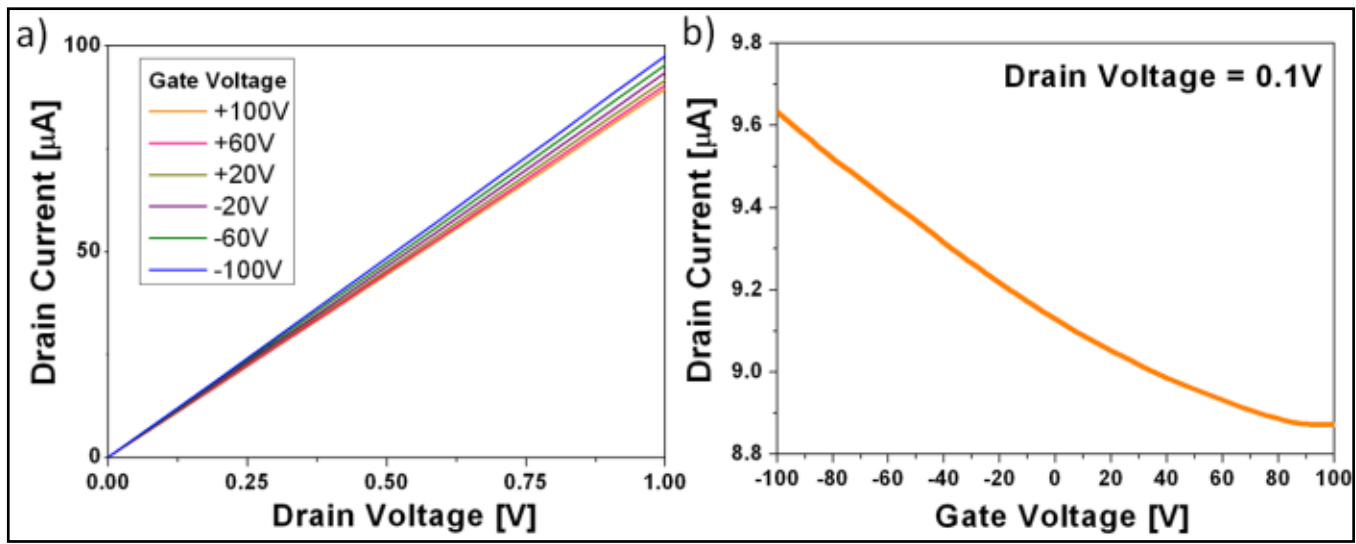

Figure 60: Output characteristics $I_{d}-V_{d}$ (drain current- drain voltage) of the transistor device under different applied gate voltages and Id-Vg (drain current-gate voltage) transfer curve of bottom-gated graphene FET under sweeping $V_{g}$ 
The hole mobility was then extracted from the linear regime of the transfer characteristics using the equation $\mu=\left(L / W C_{o x} V_{d}\right)\left(\Delta I_{d} / \Delta V_{g}\right)$ where $\mathrm{L}$ and $\mathrm{W}$ were the channel length and width, Cox the gate capacitance (which was $1.15 \times 10^{-9} \mathrm{~F} / \mathrm{cm}^{2}$ for agate oxide of $\left.300 \mathrm{~nm}\right), \mathrm{Vd}, \mathrm{Id}, \mathrm{Vg}$ were drain-source voltage, drain-source current and gate voltage, respectively. The mobility of a typical 2-hour CVD devices was extracted to be $\sim 191 \mathrm{~cm}^{2} /(\mathrm{V} . \mathrm{s})$. This value was larger than reported field effect transistor mobilities of $\mathrm{rGO}^{21,36}$ thus substantiating that the ethanol CVD treatment and rGO growth allowed 2D charge percolation of the extended $\pi$-electron network.

In summary, atmospheric pressure ethanol CVD had been demonstrated to reduce oxygen moieties and extend the size of GO. The GO catalysed the deposition of carbon and this growth extension was observed by the SEM and characterized to be rGO using Raman and XPS. Raman spectra indicated that the ethanol grown rGO film possesses lower defect density, better than thermally reduced or hydrazine reduced rGO. This approach reduced electrical resistance and decreased the standard deviation between chips. This finding suggested that the electrical conductivity was not limited by the presence of defects in the form of carbon vacancies, which could not be healed by annealing or reduction. To the best of knowledge, these results constituted the first example of using GO for growth extension. In a broader perspective, the possibility of GO growth represented a big step forward to fabrication of practical and usable electronics.

\subsection{MECHANISM OF RGO GROWTH}

In theory, any hydrocarbon source such as methane, ethylene and ethanol could be used. However, liquid carbon sources such as ethanol were a safer alternative than gaseous hydrocarbons, which were extremely flammable and may form explosive mixtures with air. Thus ethanol was used for the carbon source.

During the deposition phase, the gaseous hydrocarbon was thermally pyrolyzed: 
each of the C-C and $\mathrm{C}-\mathrm{H}$ bond could break to form two free radicals with each fragment keeping one electron. The presence of radical in the molecule allowed rapid rearrangement of carbon bonds. Though the reaction was more complicated for alcohols, the possible cracking of carbon radical species to form carbon deposition in the basic form could be expressed as Equation 5 and the catalytic hydrogenation ${ }^{294}$ process of solid carbon in which $\mathrm{H}_{2}$ formed $\mathrm{CH}_{4}$ as Equation 6.

Equation 5: Cracking of carbon radical species

$$
\mathrm{C}_{\mathrm{x}} \mathrm{H}_{\mathrm{y}}(\mathrm{g}) \leftrightarrow x \mathrm{C}(\mathrm{s})+(\mathrm{y} / 2) \mathrm{H}_{2}(\mathrm{~g})
$$

Equation 6: Catalytic hydrogenation of solid carbon

$$
\mathrm{C}(\mathrm{s})+2 \mathrm{H}_{2}(\mathrm{~g}) \rightarrow \mathrm{CH}_{4}(\mathrm{~g})
$$

According to Le Chatelier's principle and Equation 5, the growth rate of carbon will decrease with decreasing hydrocarbon concentration and increasing hydrogen gas. From Equation 6, increasing the ratio of hydrogen gas increased the dissociation rate of carbon and hydrogen into gaseous hydrocarbons. Thus the addition of hydrogen gas could help to etch away amorphous or excess carbon formed $^{12}$. In the experiment, by increasing the hydrogen to argon gas ratio from 0.17 to 0.34 while maintaining the total flow volume of gas mixture, no deposition of carbon occurred even after 1 hour. With the ethanol precursor removed from the system for only 1 hour, no carbon Raman signature was detected on the substrate; the entire GO template was etched away. Thus, the deposition of rGO in the experiment obeyed the Le Chatelier's principle as depicted in Equation 5 and occurs in direct competition with the catalytic hydrogenation process as depicted in Equation 6. 
In Figure 61, existing rGO flakes were highlighted in purple and the new growths were highlighted in yellow. Figure 61a showed the GO coverage of ca. $60 \%$ after a typical APTES-functionalization and $\mathrm{GO}$ incubation on $\mathrm{SiO}_{2}$. However, after a partial ethanol CVD treatment of 30 minutes, the coverage increased to ca. $80 \%$ as shown in Figure $61 \mathrm{~b}$. A high magnification of the new growth was shown in Figure 61c.

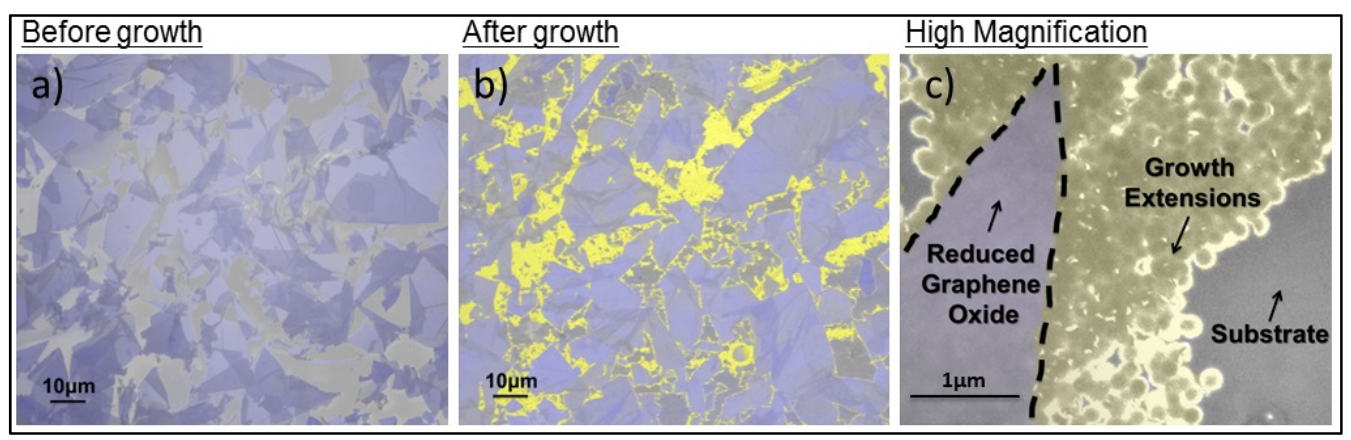

Figure 61: Scanning electron microscope image of (a) before ethanol CVD treatment GO flakes with ca. $60 \%$ coverage and (b) after 30-minutes ethanol CVD treatment rGO flakes with ca. $80 \%$ coverage [GO flakes highlighted in purple and new growth highlighted in yellow, please refer to online version for colour] (c) High-magnification image on the new growths.

It was shown in the high-resolution SEM image that the new rGO new growth continued to act as a catalyst for subsequent growth, subsequently covering more of the gap between existing rGO flakes. To prove the role of GO as an effective catalyst, substrates in the same conditions (clean and APTESfunctionalized) without GO were processed under the same ethanol CVD conditions for up to 2 hours. Neither carbon deposits nor carbon spectrum were detected by SEM and Raman respectively ${ }^{295}$. The initial GO flakes acted as crystal nucleus for the subsequent growth of these flakes ${ }^{5}$.

Since rGO could be a template for subsequent growth of rGO, this work supported the free radical condensate concept in which metal catalysts only help to initiate carbon precipitation and rearrangement at the nucleation stage: once the carbon structure was created, e.g. 1-dimensional carbon nanotube 
structure $^{296}$, the metal catalyst becomes non-functional. The carbon radicals from the pyrolysis of ethanol could then catenate into longer chains by forming covalent bonds directly on the existing edges of rGO template resulting in the growth observed ${ }^{297}$. It had been reported that the energetics of the primary particles dictated the new growth's phase; if the precursor was metastable, the growth will follow a metastable phase ${ }^{298}$. This could explain the growth of rGO during an extended ethanol CVD post-treatment on GO.

To test the ethanol CVD processed rGO electrical properties, back-gated FieldEffect Transistors based on 1 hour ethanol CVD processed GO sheets were fabricated and their electrical properties tested under vacuum at room temperature. The channel length was $100 \mu \mathrm{m}$ to ensure transport was bulklimited and role of the all contacts minimized. Figure 62a showed the drain current $\left(\mathrm{I}_{\mathrm{d}}\right)$ versus drain voltage $\left(\mathrm{V}_{\mathrm{d}}\right)$ curve at six discreet gate voltages $\left(\mathrm{V}_{\mathrm{g}}\right)$.

The graph showed linear output behaviour indicative of a good ohmic contact between the GO film and electrodes. Figure $62 \mathrm{~b}$ illustrated the same transistor $I_{d}$ under sweeping $V_{g}$ at fixed $V_{d}=0.1 \mathrm{~V}$. The threshold voltage was shifted to the positive side due to $\mathrm{p}$-doing from persistent moisture and oxygen. The CVD treated GO device showed a clear increase in conductance as the $\mathrm{V}_{\mathrm{g}}$ deviates from $0 \mathrm{~V}$, indicating that the $\mathrm{rGO}$ films behaved as ambipolar semiconducting material with stable electrical characteristics up to $\pm 100 \mathrm{~V}$. 


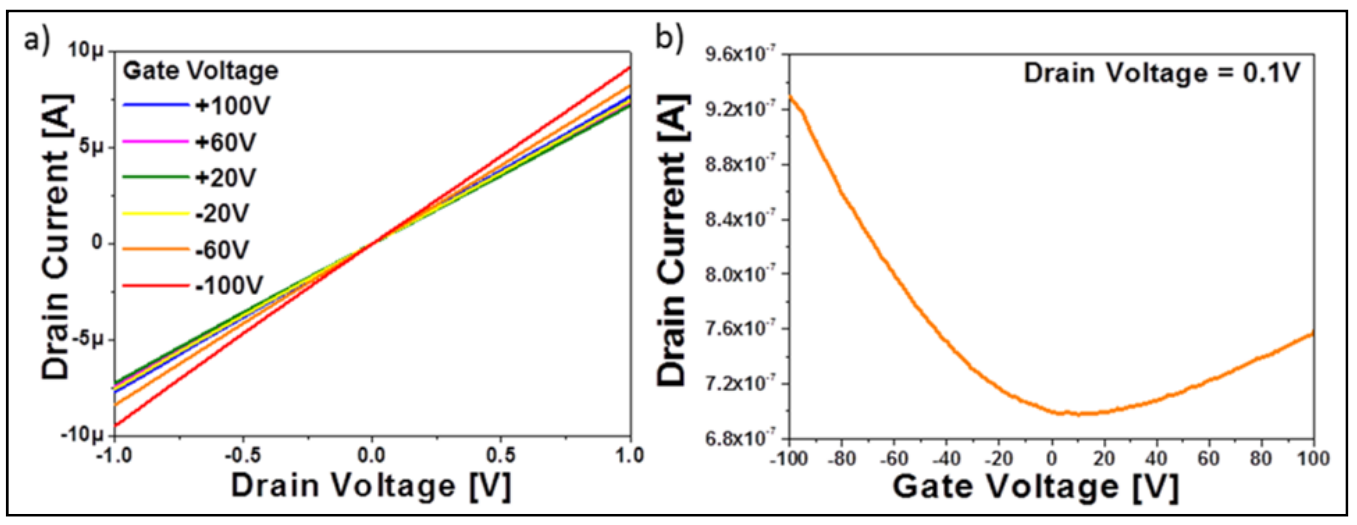

Figure 62: (a) Output graph $I_{d}-V_{d}$ (drain current- drain voltage) under different applied gate voltages and (b) $I_{d}-V_{g}$ (drain current-gate voltage) transfer curve of the CVDprocessed rGO device under vacuum and room temperature. [Please refer to softcopy version for colour]

The UV Raman spectra (Figure 63) were fitted with Lorentzian to obtain the positions and intensities of the peaks. UV Raman was used because in visible Raman, the $\mathrm{sp}^{2}$ sites had such a high cross section that they dominate the spectra. The presence of G peaks in UV excitation of GO, rGO and HOPG showed that the $\mathrm{sp}^{2}$ vibrations still dominate even in the UV Raman excitation. The diamond sample exhibited a characteristic diamond peak at $1330 \mathrm{~cm}^{-1299}$. For CVD processed rGO, the D peak was measured at $1409 \mathrm{~cm}^{-1}$ and $\mathrm{G}$ peak at $1584 \mathrm{~cm}^{-1}$ in agreement with existing literature ${ }^{5,300,301}$.

The grown carbon could either be rGO-like carbon or diamond-like carbon (DLC). RGO was defined generally as $\mathrm{sp}^{2}$ regions in a $\mathrm{sp}^{3}$ matrix whereas DLC was defined as amorphous carbon with $\mathrm{sp}^{3}$ and trans-polyacetylene bonds ${ }^{300}$. The $\mathrm{T}$ peak ${ }^{300}$ that was due to C-C $\mathrm{sp}^{3}$ vibrations occured only with UV Raman excitation at wave number of $\sim 1060 \mathrm{~cm}^{-1}$. It was due to a resonant enhancement of the $\sigma$ states and it directly probed the $\mathrm{sp}^{3}$ bonding. As the $\mathrm{sp}^{3}$ content fell, the $\mathrm{sp}^{3}$ vibrational density of states peak $1060 \mathrm{~cm}^{-1}$ will shift upwards to that of $\mathrm{sp}^{2}$ VDOS at $1400 \mathrm{~cm}^{-1300}$. In both GO and CVD processed rGO spectra, no T peak was observed, indicating that the CVD treated samples' $\mathrm{sp}^{3}$ percentage was small and non-diamond like. It was also observed that the D peak decreased as the CVD processing time increased, indicating increasing graphitic order in the rGO structure ${ }^{302}$. 


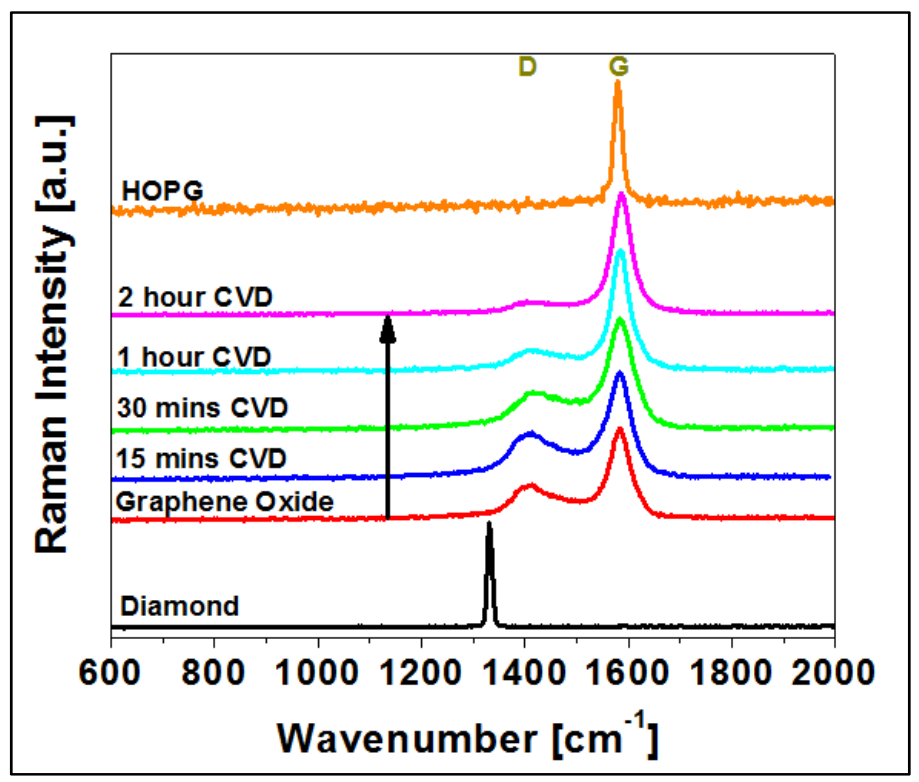

Figure 63: Ultra-violet Raman spectra of diamond, GO, different ethanol CVD processed rGO and HOPG. The intensities of diamond and HOPG were scaled down to allow fitting into graph

The $\mathrm{I}_{\mathrm{D}} / \mathrm{I}_{\mathrm{G}}$ ratio of the UV Raman (Table 15) was used to estimate the $\mathrm{sp}^{2}$ domain size using the Tuinstra-Koenig empirical formula ${ }^{303}$ in UV conditions ${ }^{17}$, 300 . The ratio decreased continuously from 0.42 in the GO sample to 0.09 in the 2 hour CVD treated samples indicating increased ethanol CVD treatment resulted in increased $\mathrm{sp}^{2}$ domain size in the rGO samples. Thus from Raman spectrums and $\mathrm{I}_{\mathrm{D}} / \mathrm{I}_{\mathrm{G}}$, it was confirmed that the carbon growth to be $\mathrm{rGO}$, that the CVD process increased graphitization and that the $\mathrm{sp}^{2}$ domain size increased with increasing processing time. 
Table 15: D/G Raman spectrum peak intensity, AFM height profile and 4-point probe sheet resistance for GO and rGO reduced under different conditions

\begin{tabular}{|c|c|c|c|c|c|c|c|}
\hline & & GO & $\begin{array}{c}15 \text { mins } \\
\text { CVD }\end{array}$ & $\begin{array}{c}30 \text { mins } \\
\text { CVD }\end{array}$ & $\begin{array}{l}1 \text { hour } \\
\text { CVD }\end{array}$ & $\begin{array}{c}2 \text { hour } \\
\text { CVD }\end{array}$ & $\begin{array}{c}12 \text { hour } \\
\text { hydrazine } \\
39\end{array}$ \\
\hline $\begin{array}{c}\text { UV Raman } \\
\text { Spectroscopy }\end{array}$ & $\begin{array}{c}\text { Peak intensity of } \\
\text { D/G }\end{array}$ & 0.42 & 0.35 & 0.32 & 0.17 & 0.09 & NA \\
\hline AFM Height & $\begin{array}{l}\text { Flake thickness } \\
\qquad(\mathrm{nm})\end{array}$ & 1.4 & 1.5 & 2 & 4 & 4 & NA \\
\hline Profile & $\begin{array}{l}\text { New growth } \\
\text { thickness (nm) }\end{array}$ & 0 & 0 & 0.8 & 1 & 2 & NA \\
\hline 4-point probe & $\begin{array}{c}\text { Average } \\
\text { Resistance }(\Omega / \square)\end{array}$ & NA & $6.10 \mathrm{E}+4$ & $2.87 \mathrm{E}+4$ & $2.23 \mathrm{E}+4$ & $4.68 \mathrm{E}+3$ & $1.99 \mathrm{E}+6$ \\
\hline $\begin{array}{c}\text { Sheet } \\
\text { Resistance }\end{array}$ & $\begin{array}{c}\text { Standard } \\
\text { Deviation }(\Omega / \square))\end{array}$ & NA & $4.43 \mathrm{E}+4$ & $1.25 \mathrm{E}+4$ & $7.16 \mathrm{E}+3$ & $7.72 \mathrm{E}+2$ & $1.60 \mathrm{E}+6$ \\
\hline$(n=5)$ & $\begin{array}{c}\text { Relative Standard } \\
\text { Deviation }(\%)\end{array}$ & NA & 72.6 & 43.6 & 32.1 & 16.5 & 80.5 \\
\hline
\end{tabular}

From 0 to 15 minutes of ethanol CVD treatment, the AFM height profile indicated that the thickness of pre-existing GO template had negligible increase in height and no growth from the edges was observed ${ }^{12}$. However, as the process time increased to 30 minutes, new growths appeared and the thickness of the GO template also increased slightly by $0.5 \mathrm{~nm}$. With increasing process time to 2 hours, the new growths continued to extend outwards and completely cover the gaps in between the pre-existing rGO flakes. The new growth thickness after 2 hours of growth was $2 \mathrm{~nm}$ and this corresponded to $\sim 2-3$ layers of $\mathrm{rGO}^{39}$.

From the 4-point probe measurement, the average electrical resistance of the chips decreased from $1.99 \mathrm{E}+6$ to $4.68 \mathrm{E}+3 \Omega$ /square $(99.76 \%$ reduction) while the relative standard deviation decreased from $1.60 \mathrm{E}+06$ to $7.72 \mathrm{E}+02 \Omega$ /square 
(99.95\% reduction) after a 2 hour ethanol CVD treatment as compared to 12 hour hydrazine vapor reduction. The decrease in electrical resistivity in the ethanol CVD treated samples was attributed to the increased percolation pathways between flakes due to the new growth completely filling up gaps between pre-existing rGO flakes. Due to the more uniform coverage and increased coverage of the rGO on the substrate, the reported growth method had decreased the standard deviations between chips, thus solving a critical problem for the practical use of rGO in practical electronic devices.

Having considered the data from the experiment, the growth sequence of rGO schematic was described (Figure 64). Ethanol CVD treatment of up to 15 minutes on the GO lead to an improved graphitization from the free radicals produced from pyrolysis of gaseous ethanol. After 30 minutes, a thin graphitic shell was formed around the GO catalyst. Due to the lower growth activation energy at the edge of graphene sheets for sequential addition of carbon hexagons ${ }^{304}$, rGO preferentially grew out from the edges. Finally, rGO completely covered the substrate after 2 hours of the CVD ethanol growth process. 


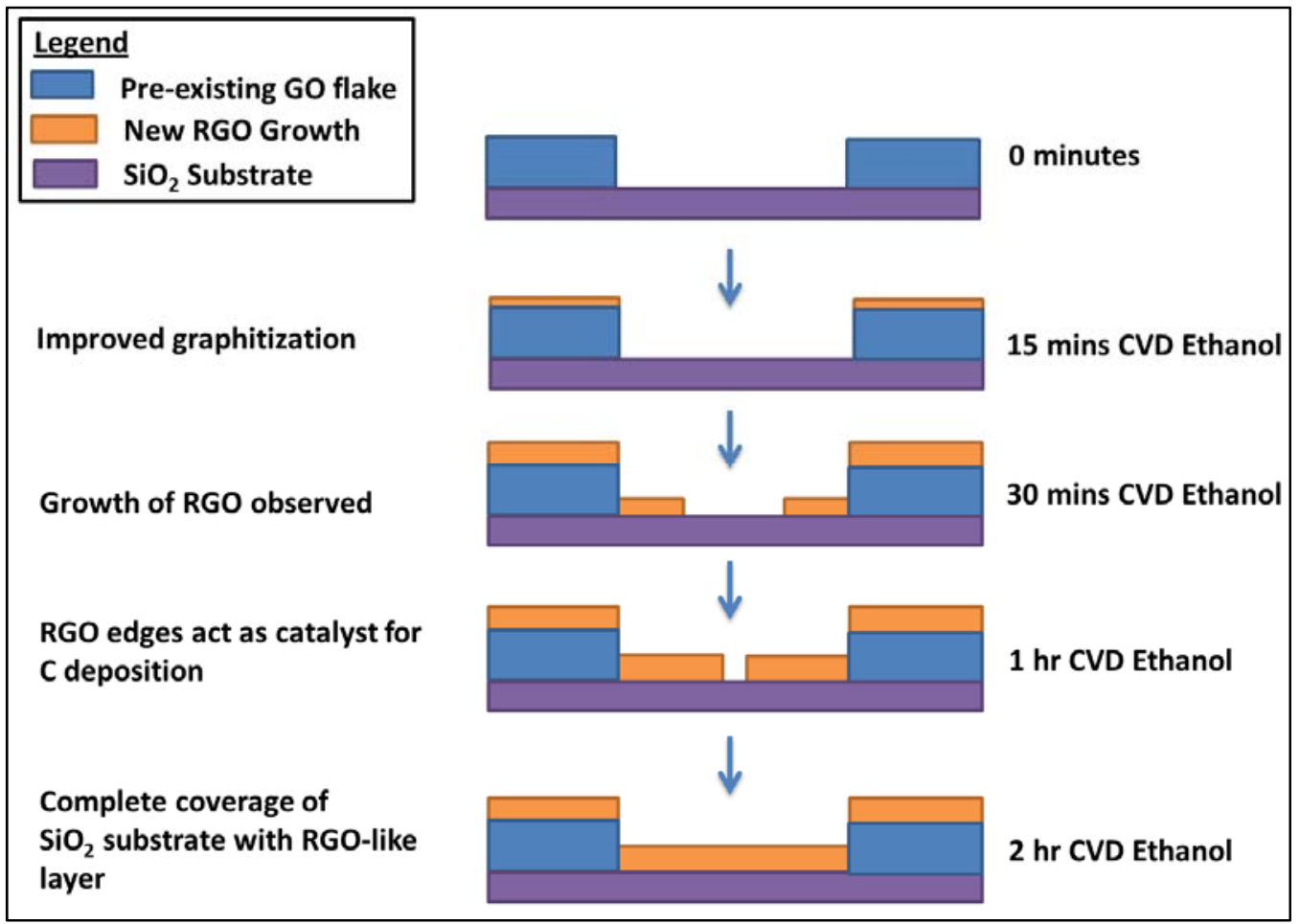

Figure 64: Schematic showing the growth sequence of rGO from a GO template during ethanol CVD treatment

The presented data suggested that $\mathrm{rGO}$ could be grown via the free radical condensate mechanism. The mechanism of carbon nanotube carbon growth had been debated and two different mechanisms had been proposed: the dissolution of elemental carbon into metal nano-clusters followed by precipitation ${ }^{305}$ or the free radical condensate that carbon could act as reaction medium for production of nanotubes $^{294}$. In the experiment, no metal catalyst was used, but rGO growth was observed, thus supporting the free radical condensate growth theory. After the pyrolysis of ethanol, free radicals preferentially condensated at the edges of the pre-existing GO flakes and thus rearranged itself into a new carbon skeleton until a local minimum had been reached. 


\section{$\underline{6.5 \text { CONCLUSION }}$}

This chapter reported that the complete coverage of $\mathrm{rGO}$ on $\mathrm{SiO}_{2}$ substrate was achieved using GO template under ethanol CVD at ambient pressure without any metal catalysts. GO edges preferentially acted as a catalyst for further growth of rGO. The growth sequence supported the free radical condensate growth theory that carbon could nucleate and grow without a metal catalyst. After a 2 hour ethanol CVD treatment, the electrical resistivity decreased by $99.8 \%$ and its relative standard deviation decreased by $79.5 \%$ compared to hydrazine treated samples. The results and proposed mechanism show that the use of 2 hour CVD treatment on GO was a promising way towards a better and homogenous rGO for practical, high-yielding preparation of novel functional biosensors, electronics, batteries or crystal growth templates. 


\section{CHAPTER 7: DEVELOPMENT OF NOVEL \\ BIOSENSOR FOR IL-6 DETECTION AND \\ Determining its Presence in Sweat}

This chapter described the rationale and development of a novel biosensor using carbon-based transducer. The detection of Interleukin (IL-6), a protein circulating in the body at very low concentration $(\mathrm{pg} / \mathrm{ml})$, was reported. Large ethanol chemical vapor deposition-processed GO, obtained from previous chapters, was fabricated into liquid-gated field effect transistor biosensors. The biosensor was able to operate in the ultra-sensitive IL-6 physiological-relevant range.

The chapter then described a study to determine the possibility of measuring IL6 non-invasively in real and artificial sweat using an enzyme-linked immunosorbent assay (ELISA). Although ELISA measurement was capable of detecting IL-6 in artificial sweat at concentrations well below the normal plasma physiological concentration, no IL-6 was detected in human subject participants either at rest in a thermally challenging environment, or following exercise.

\subsection{INTERLEUKIN-6 BIOSENSOR}

Interleukin-6 (IL-6) was a pleiotropic cytokine with an important role in both immune regulation and exercise metabolism. During exercise, IL-6 was predominantly produced within, and released from, the working skeletal muscle, with the magnitude of IL-6 release related to the duration and intensity of the exercise bout. IL-6 was the first cytokine to appear in circulation following initiation of exercise and undergoes the most pronounced increase as compared to any cytokine in response to exercise. In the last decade, studies 
had suggested a role for IL-6 as a muscle energy sensor, pointing to its potential role as a biomarker of overtraining.

Furthermore, it had been reported recently that there was no difference in the measurement of plasma IL-6 using the more invasive venous versus the minimally invasive capillary blood sampling measurement. The possible use of capillary measurement meant a minimally invasive way to quantitatively monitor changes in plasma IL-6 levels ${ }^{306}$. The use of capillary sampling was also a more accessible, tolerable and faster way for field-based blood measures during sport providing an ideal route for point-of-care testing. Thus the IL-6 protein had immense potential as a training and fatigue biomarker if it could be measured in real-time.

Currently, ELISA and western blot was the staple detection technique for IL$6^{307}$. However, they require substantial time, cost, machinery and specialist training; thus making real-time detection of IL-6 impossible. In contrast, a liquid-gated FET biosensor offered real-time monitoring and required only a single antibody to capture the antigen and report a signal. When the target antigen binds to the antibody, it changes the environment near the surface of the transducer and alters its electrical conductance ${ }^{308}$.

Graphene oxide (GO) was analogous to graphene with oxygen moieties and had the distinct advantages of being solution processable, industrially scalable and a tuneable electronic band-gap ${ }^{256,287}$. Due to the monolayer configuration of GO, all its carbon atoms were exposed to the environment and the charge-carriers were confined to the surface of the sheets. Thus the electrical conductivity of the flake was very sensitive to local electrostatic perturbations ${ }^{221}$. This property makes it suitable for sensing applications. GO biosensors had been reported for the detection/discrimination of amino acid tryptophan enantiomers (0.10-1.0 $\mathrm{mM})^{309}$, living organism staphylococcus aureus (single $\left.\mathrm{CFU} / \mathrm{ml}\right)^{310}$, simple sugar glucose $(0-8 \mathrm{mM})^{311}$, nucleic acid DNA $(1.0 \mathrm{fM}-0.1 \mu \mathrm{M})^{312}$, glycoprotein enzyme PSA molecules $(1.1 \mathrm{fM}-1 \mu \mathrm{M})^{313}$ and monoamine catecholamine (1mM$10 \mathrm{mM})^{11}$. 
However, the coverage of mono-layered $\mathrm{GO}$ flake on $\mathrm{SiO}_{2}$ substrate was limited due to rinsing and unwanted crosslinking of the 3AminoPropylTriEthoxy Silane (APTES) adhesion layer, thus leading to low available GO surface area, high variability of electrical conductivity between chips and low sheet transconductance that limited the sensitivity of sensors.

This limitation was overcome by depositing carbon on the edges of GO flakes using an ethanol chemical vapor deposition (CVD). Then, the post-treated GO was fabricated into a liquid-gated biosensor and the detection window for IL-6 was presented. This work yielded a highly conductive and electrically homogeneous carbon-based facile platform suitable for sensitive detection of IL-6. Then, the detection window for IL-6 was presented.

\subsection{ETHANOL CVD RGO TRANSDUCER FABRICATION}

Graphite flakes of 3-5mm were obtained from NGS Naturgraphit $\mathrm{GmbH}$, Germany. All the chemicals (research grade) were obtained from Sigma Aldrich and Merck. IL-6 protein and antibody were obtained from BD (ELISA kit No. 555220). Graphene oxide (GO) sheets were prepared by the modified Hummers' method from natural graphite flakes as reported ${ }^{5,39}$. Briefly, graphite flakes were pre-oxidized using ultra-sonication and then oxidized using $\mathrm{H}_{2} \mathrm{SO}_{4}$ $(120 \mathrm{ml})$ and $\mathrm{KMnO}_{4}(15 \mathrm{~g})$. Then $\mathrm{H}_{2} \mathrm{O}_{2}(20 \mathrm{ml})$ was added into the mixture. Finally, the resulting floating GO particles were scooped out and stirred overnight in DI water $(500 \mathrm{ml})$ to obtain a stock solution of GO. This solution was then centrifuged at 3000xg for 5 minutes and further purified by dialysis (cut-off 3000MW) against DI water for one week.

Then, silicon dioxide chips were silanized using APTES and the GO solution was drop-cast. The silanization enabled a mono-layer of GO to be electrostatically attached. The chips were then placed in an atmospheric pressure ethanol CVD furnace and processed at $950^{\circ} \mathrm{C}$. In the $\mathrm{CVD}, \mathrm{H}_{2}$ $(20 \mathrm{sccm})$ and Ar $(100 \mathrm{sccm})$ gas were mixed and passed through an ethanol bubbler before entering the furnace. Finally, the chip was then fabricated into a 
liquid-gated FET biosensor with 1-pyrenebutanoic acid, succinimidyl ester (PBSE) linker, IL-6 antibody with blocking agents bovine serum albumin and ethanolamine.

\subsection{IL-6 BIOSENSOR CHARACTERIZATION AND TEST}

According to Lerf-Klinowski's GO model, carboxyl (-COOH) groups were found at the edges of $\mathrm{GO}^{36,314,315}$. Thus an etching action by $\mathrm{H}_{2}$ was added to activate the carbon sites, followed by possible carbon deposition at the edges. Scanning Electron Microscopy (SEM) was performed and the image of GO before growth (Figure 65), after a 30-minute CVD treatment growth (Figure $65 \mathrm{~b})$ and a high magnification image of the carbon growth observed near the edges of GO (Figure 65c) were shown. In the images, the GO flakes were highlighted in purple and the new carbon growths were highlighted in yellow.

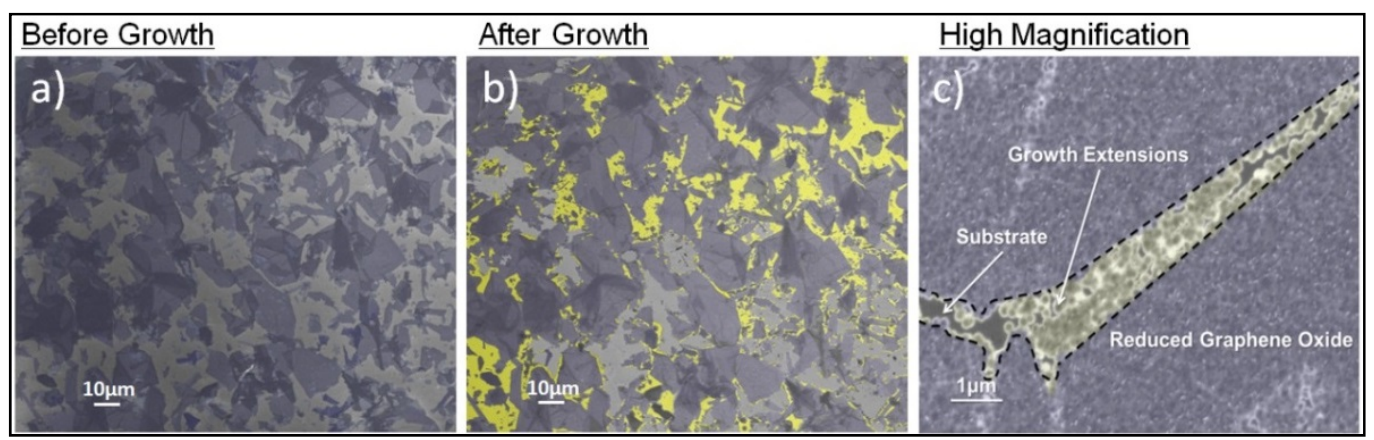

Figure 65: SEM images of (a) GO flakes before ethanol CVD treatment with ca. 60\% coverage (b) reduced GO flakes after 30-minutes CVD treatment with ca. $75 \%$ coverage in total. (c) high-magnification on the new growths between two reduced GO flakes. [GO flakes and new carbon growths were highlighted in purple and yellow respectively, please refer to online version for colour]

The ethanol CVD-treated reduced GO chips were then fabricated into liquid gated FETs (Figure 66a and b) in order to test their bio-sensing capability. Silicone rubber well was used to hold the liquid analyte in place during sensing. Silicone rubber had good electrical insulating and sealing properties. A sensing 
fluidic channel was fabricated using polydimethylsiloxane (PDMS) mould and attached for continuous monitoring.
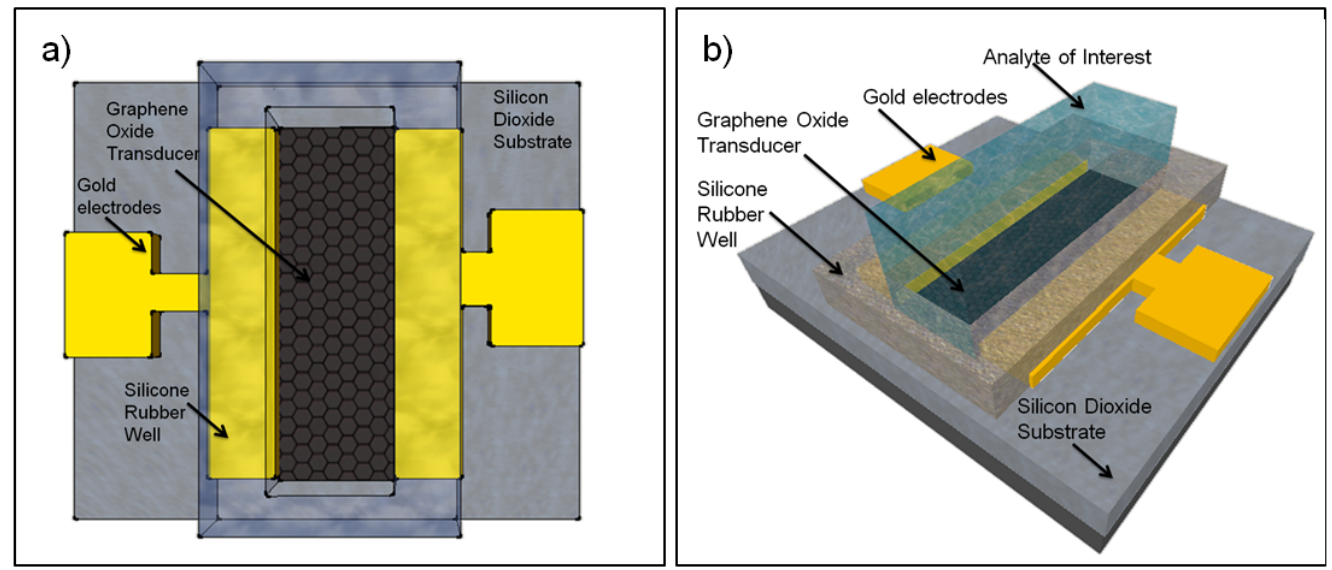

Figure 66: Schematic showing (a) top-view and (b) side-view of fabricated FET devices with silicon well suitable for analyte testing

There were many types of blocking buffer such as skim milk, Tween-20, PEG and BSA. It had been reported in many graphene literatures that BSA was adequate in passivating the graphene transducer against any non-specific binding via physical adsorption ${ }^{316-318}$. In this experiment, BSA was used to bind to the unreacted 1-Pyrene butanoic acid on the surface of graphene surface in the experiment via covalent attraction ${ }^{319}$.

The shift in source-drain current $\left(\mathrm{I}_{\mathrm{SD}}\right)$ of the anti-IL-6 immobilized rGO transistor was sensitive to increasing concentrations of IL-6 protein due to electronic n-doping of the device. Figure 67a showed that binding of increasing amounts of IL-6 protein to the anti-IL-6 antibody resulted in a down-shift of the $\mathrm{I}_{\mathrm{SD}}-\mathrm{V}_{\mathrm{G}}$ curve, from $21.09 \mu \mathrm{A}(0 \mathrm{pg} / \mathrm{ml})$ to $20.96 \mu \mathrm{A}(300 \mathrm{pg} / \mathrm{ml})$ at $\mathrm{V}_{\mathrm{G}}=0 \mathrm{~V}$.

Low gate voltages were used to minimize possible disturbances to the biological sample and prevent instability of the biosensor due to electrochemical and charging effect ${ }^{11}$. The sensor was operating at the p-type region at $\mathrm{V}_{\mathrm{G}}=0 \mathrm{~V}$ and the negative shifts were tentatively attributed to the electrostatic gating effect on the rGO surface. The increasing positive charge 
from the immune-complex formation could induce negatively charges in the rGO thus n-doping (down-shifting) the $\mathrm{I}_{\mathrm{SD}}-\mathrm{V}_{\mathrm{G}}$ curve $\mathrm{e}^{308,320}$. It could also be due to a decreased ionic screening effect after the protein-antibody interaction ${ }^{321,322}$. The specific doping mechanism during biomolecular interactions on 2D carbon liquid-gated FET sensing surface was still not fully understood and required further study ${ }^{313,320}$.

The decrease in $\mathrm{I}_{\mathrm{SD}}$ corresponded to a sensitivity of $1.5 \mathrm{pA}$ per $\mathrm{pg} / \mathrm{ml}$ of IL-6. The slope of the $\mathrm{I}_{\mathrm{SD}}-\mathrm{V}_{\mathrm{G}}$ curve did not change thus indicating that the charge carrier mobility in the rGO transducer remained the same after the proteinantibody reaction. It was noted that the $\mathrm{I}_{\mathrm{SD}}$ downshift (electrostatic gating effect) exhibited in the reported biosensor was more reproducible and reliable than other effects using Schottky barrier and changes in gate coupling and carrier mobility ${ }^{308}$.

The change in $\mathrm{I}_{\mathrm{SD}}$ could then be utilized to be a sensor for the IL-6 concentration by fixing the gate voltage value. The general trend of the $\mathrm{I}_{\mathrm{SD}}$ decreasing with increasing concentration of IL-6 was evident in the calibration curve (Figure 67b). The linear dynamic range (LDR) of the sensor was from 4.7 to $18.8 \mathrm{pg} / \mathrm{ml}$ of IL-6. Although at higher concentrations, the change in the sensor electrical signal was not much higher than at $18.8 \mathrm{pg} / \mathrm{ml}$, but the sensor still acted as an active material that gave a reliable reading against IL-6 concentrations (Figure $67 \mathrm{~b}$ insert). The slightly non-linearity relationship between interleukin- 6 concentration and the decrease of measured source-drain current could be attributed to the carrier injection during the $I-V$ measurement ${ }^{228}$. It had also been commonly observed for carbon-based FET biosensors $^{11,228,323-325}$. Further studies were needed to reveal the details of the sensing mechanisms. Figure $67 \mathrm{c}$ showed no change in the $\mathrm{I}_{\mathrm{SD}}$ when the sensor was exposed to interference protein, BSA. This showed the sensor resistance (after IL-6 attachment and BSA protection) in preventing interferences and unspecific binding. 


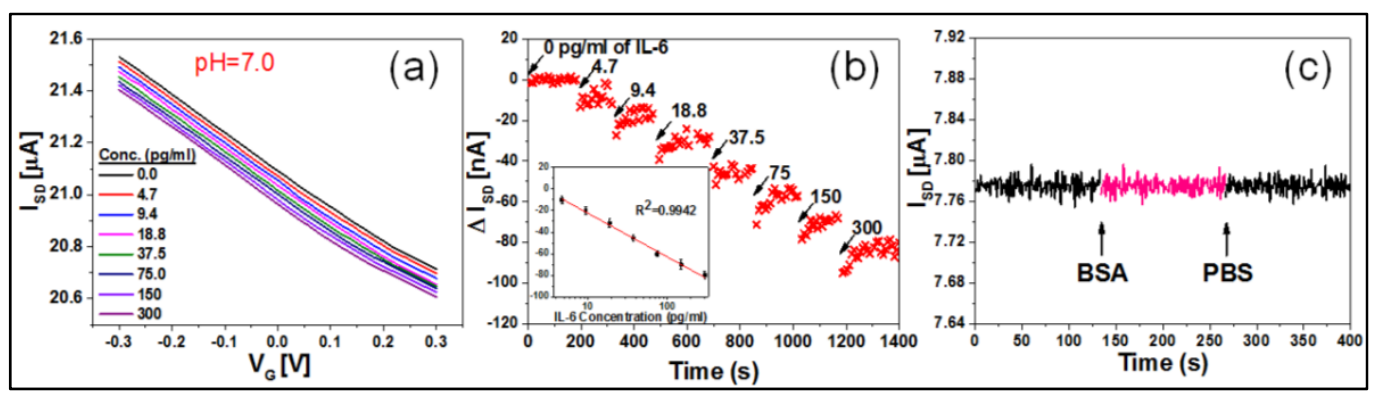

Figure 67: Detection characteristics of rGO FET biosensors. (a) Source-drain current [ $\left.I_{s d}\right]$ vs. gate voltage $\left[\mathrm{V}_{\mathrm{G}}\right]$ plot with different concentration of IL-6 protein, showing decreasing $I_{S D}$ at increasing IL-6 addition; (b) $I_{\text {SD }}$ vs. time with addition of 0-300pg/ml of IL-6; and (c) real-time $I_{\text {SD }}$ plot, showing negligible effect when BSA interference protein was added.

The LOD of the reported IL-6 sensor based on $3 \sigma$ was $2.9 \mathrm{pA}$ or $1.53 \mathrm{pg} / \mathrm{ml}$. Plasma IL-6 levels in physically active individuals had been reported to be as low as $\leq 1 \mathrm{pg} / \mathrm{ml}^{182,326}$ while levels in sedentary middle-aged men $\sim 11 \mathrm{pg} / \mathrm{ml}$ and healthy premenopausal women had been reported in the range of $\sim 10-11 \mathrm{pg} / \mathrm{ml}$ 327 . Following prolonged exercise such as cycling, IL-6 could be elevated as much as 38 fold, and as much as 128 fold following completion of a marathon 326; thus the detection range of the reported sensor was within the physiological range of IL-6. However, further work was still needed to improve the detection limit of the sensor.

The eventual end point application would be for point of care, whole blood analysis. Thus the ability of the rGO sensor to sensitively and selectively detect the biomarker in serum demonstrates its relevance to potential usage in medical diagnostics. In this experiment, fetal calf serum was used to simulate the assay conditions.

Table 16 compared the sensitivity range of different types of biosensors to IL-6 reported recently. Using the reported label-free GO sensor, the sensitivity range was within the physiological range of $\sim 10 \mathrm{pg} / \mathrm{ml}$ in sweat samples from healthy women ${ }^{189,190}$ which were not to be significantly different from plasma levels ${ }^{189}$. 


\section{Reference Transducer \\ Platform \\ Sensitivity \\ range \\ $(\mathrm{pg} / \mathrm{ml})$}

Note

4.7-300 Label-free

Reported Post-processed GO FET immunoassay 4.7-300 Label-free

325

SWCNTs/AuNPs
Electrochemical impedance immunoassay

$1 e^{-5}-0.1$

Label-free

Surface plasmon resonance (SPR) immunoassay

0.78 to $12.5 \quad$ Label-free Biacore sensor chip

Microfabricated

329 silicon flexural plate wave (FPW) resonators

Microelectromechanical (MEM) 1.2-unknown Label-free immunoassay

$330 \quad$ Fiber-optic dipprobe Optical
immunoassay

$120-12000$ Label-free

Electrochemical immunoassay

$0.002-20$

Porous 331 Polymeric
nanoparticles on
calcium carbonate

$$
332
$$
Modified carbon electrodes
333 Functionalized fluorescent core- shell nanoparticles Electrochemical
immunoassay

$1-1000$

PS@PDA-metal label used

Carbon Nanotube
Forest Electrodes
Fluoro- immunoassay

Fluorescent core-shell nanoparticles label used

Electrochemical
immunoassay
20-1250

$0.5-5$
Ab2-MWNTHRP label used

The two distinct goals in successful biosensing was the determination of small quantity of specific protein biomarker and the other was the validation or discovery of biomarkers, with the latter expected to accelerate early screening 
of high risk populations at point-of-care. The next section set forth to validate the presence of IL-6 in sweat and if it could be correlated to the circulation IL-6.

\subsection{PRESENCE OF IL-6 IN SWEAT}

A study was conducted to determine the possibility of measuring IL-6 in sweat using an enzyme-linked immunosorbent assay (ELISA) and investigate the relationship between plasma- and sweat-derived measures of IL-6. For this section, the data reported were not obtained alone by the author. The author was part of a group working on this study together. The experiments were performed in Loughborough University.

IL-6 concentrations were closely related to both increased feelings of fatigue at rest $^{177}$ and impaired running performance ${ }^{178}$. Therefore, the measurement of IL6 throughout the course of training may be beneficial to the management of training load and athletic performance in elite athletes.

The literature had reported non-invasive detection of cytokines in body fluids from saliva ${ }^{188,334,335}$ and sweat ${ }^{190,327}$ with varying degrees of success. Saliva had been reported to be a poor medium for cytokine measurement, as there was no correlation between IL-6 concentrations in blood versus saliva either at rest $^{334}$ or following strenuous exercise ${ }^{188}$.

However, sweat may be a more promising medium for non-invasive measurement of systemic IL-6. Marques-Deak et al. ${ }^{327}$, collected sweat from females over a $24-\mathrm{h}$ period and reported that IL-6 was present in measurable concentrations, above those of plasma IL- $6^{190,} 327$. Importantly, the IL-6 concentrations detected in sweat and from venous plasma were correlated and the concentrations appeared comparable ${ }^{327}$, suggesting that sweat may be a potential vehicle for biomarker measurement. However, although some other groups had been successful in measuring other cytokines including IL-1 $\alpha$, IL-1 $\beta$ and IL- 8 in sweat in response to exercise ${ }^{336}$, to the author's knowledge, no other groups had successfully reported the presence of IL-6 in sweat at rest or in response to exercise in healthy individuals. 
A subsequent small-scale proof of concept study aimed to determine whether IL-6 was present in sweat, and to examine the relationship between venous and sweat collection methods for IL-6 measurement at rest and in response to exercise. It was hypothesised that IL-6 would be detectable in sweat and that this would be correlated to IL-6 concentrations found in venous blood plasma.

\subsubsection{MEASUREMENT AND TRIAL METHODS}

\section{ELISA Measurement Method}

All incubations were carried out at room temperature on a horizontal orbital shaker at $1100 \mathrm{rpm}$ unless stated otherwise. Wash steps were carried out with $0.05 \%$ Tween-20 in tris-buffered saline (TBS-T).

96-well Immulon 4HBX ELISA plates (Fisher Scientific) were coated overnight at $4{ }^{\circ} \mathrm{C}$ with $100 \mu \mathrm{L}$ anti-IL-6 monoclonal capture antibody, diluted 1:250 in sodium carbonate buffer ( $\mathrm{pH}$ 9.5). The following morning, plates were washed and blocked for $1 \mathrm{~h}$ with $5 \%$ bovine serum albumin (BSA) in trisbuffered saline (TBS). Following this step, plates were washed and $100 \mu \mathrm{L}$ samples or standards were added to wells in duplicate. The standard curve consisted of a double dilution series (concentration range: $0.31 \mathrm{pg} / \mathrm{mL}$ to 20 $\mathrm{pg} / \mathrm{mL}$ ) of recombinant human IL-6 (rhIL-6) diluted in TBS with $10 \%$ foetal calf serum (FCS). Plasma samples were diluted 1:2 in TBS/FCS and incubated for $2 \mathrm{~h}$, while sweat samples were treated with $10 \% \mathrm{FCS}$ and added to the wells neat. After washing, $100 \mu \mathrm{L}$ biotinylated detection antibodies, diluted 1: 250 in TBS-T with 1\% BSA, was added to each well and incubated for $1 \mathrm{~h}$. Following washing, $100 \mu \mathrm{L}$ streptavidin-alkaline phosphatase, diluted 1:2000 in TBS with $1 \%$ BSA, was added to wells. After incubating for $45 \mathrm{~min}$, an ELISA amplification system (Invitrogen) was used to develop colour in wells according to the manufacturer's instructions. The amplification reaction was stopped with $10 \%$ hydrochloric acid. Absorbance in wells was read at $490 \mathrm{~nm}$ on a Varioskan plate reader, with $650 \mathrm{~nm}$ as the correction wavelength. Intraassay coefficients of variation $(\mathrm{CVs})$ were $<5 \%$. 


\section{Sweat collection Method}

Sweat samples were collected using a disposable sweat collection method ${ }^{337}$. Briefly, a sweat patch consisting of laboratory film paper (Parafilm, American Can Co.) was attached to the adhesive side of a sterile wound dressing (Opsite, Smith \& Nephew) to form a collection chamber once applied to the skin surface. The skin was cleaned with an alcohol swab before the collection patches were attached to the left and right scapular and further secured with tape (Transpore, $3 \mathrm{M}, \mathrm{UK})$. The sweat collection area was $12 \mathrm{~cm}^{2}$, with sweat later extracted using a blunt fill needle (BD, Oxford, UK) and 5-ml syringe (BD, Oxford, UK) on two occasions: i) following a 20 -min washout period and ii) on completion of exercise. Collected sweat was aliquoted into pre-weighed eppendorfs. Eppendorfs were then reweighed, centrifuged at 13,000g for $20 \mathrm{~min}$ and stored in ice before freezing at $-80{ }^{\circ} \mathrm{C}$ prior to further analysis.

\section{Preliminary trial}

Participants reported to the laboratory at 0800 hours, and measurements of body mass and height were recorded. $\mathrm{V}^{\cdot} \mathrm{O}_{2}$ peak was assessed using a continuous incremental exercise test until volitional exhaustion on an electromagnetically braked cycle ergometer. Following a 3-min warm-up period of cycling at $50 \mathrm{~W}$, workload was increased by $1 \mathrm{~W}$ every $3 \mathrm{~s}$ until volitional exhaustion. Expired air was measured using a continuous, breath-by-breath gas analyser, and heart rate was monitored throughout. 


\section{Exercise trial}

Participants reported to the laboratory at 0800 hours following an overnight fast, having avoided strenuous activity and refrained from caffeine and alcohol consumption in the previous $24 \mathrm{~h}$. A cannula was inserted into an antecubital vein to allow venous blood sampling at baseline, following $20 \mathrm{~min}$ of the trial, and immediately at the end of the trial (60 min).

Participants entered an environmental chamber set at $32{ }^{\circ} \mathrm{C}$ and $60 \%$ relative humidity, where they performed 60-min cycle ergometer exercise at a workload corresponding to $60 \% \mathrm{~V}^{\cdot} \mathrm{O}_{2}$ peak. Following 20-min exercise, the first sweat and venous blood samples were collected (washout period) and the sweat patches replaced for the remaining $40 \mathrm{~min}$ of the exercise, after which final sweat and blood samples were collected. Regional sweat rates were determined according to the change in mass of the aspirating syringe and sweat rates calculated as follows:

$$
\text { Sweat rate }\left(\mathrm{ml} \mathrm{cm}^{-2} \mathrm{~min}^{-1}\right)=\frac{\text { sweat volume }(\mathrm{ml}) \div \text { area }\left(\mathrm{cm}^{2}\right)}{\text { time }\left(\mathrm{min}^{-1}\right)}
$$

\section{Resting trial}

Participants reported to the laboratory at 0800 hours as described previously, and a cannula inserted into an antecubital vein. The skin of the scapula was cleaned, and sweat patches applied. Participants then entered an environmental chamber set at $45{ }^{\circ} \mathrm{C}, 50 \%$ relative humidity, for $60 \mathrm{~min}$. Paired venous blood and sweat samples were taken at the same time points as in the exercise trial. Regional sweat production was determined as above. 


\subsubsection{IL-6 DETECTION IN PLASMA, ARTIFICIAL AND REAL SWEAT}

To assess the capability of an ELISA to detect IL-6 in sweat, and to clarify if there was any interference of the sweat matrix on the IL-6 antibodies used in the ELISA, a set of dilution series was prepared in TBS/FCS, artificial sweat and pooled human sweat samples. Artificial sweat was prepared according to the standard reported previously ${ }^{338}$. Eight-point standard curves were set up for TBS/FCS and artificial sweat, while a three-point series spike with $10 \mathrm{pg} / \mathrm{ml}$ rhIL-6 was prepared in real sweat. Absorbance was read at 490 and $650 \mathrm{~nm}$.

The TBS/FCS and artificial sweat dilution series were assayed in quadruplicate, and the real sweat series was assayed in duplicate. Both real and artificial sweat series closely overlay on a single standard curve with a mean assay coefficient of variation of $3.6 \%$ (Figure $68 \mathrm{a}$ ).

During the resting trial, there was no detectable sweat collected during the initial 20-min washout period. In the exercise condition, following the washout period, sweat rate was $0.6 \pm 0.4 \mathrm{ml} \mathrm{cm} 2 \mathrm{~min}-1$. During the main collection period, sweat rate in the exercise trial was significantly higher than during the resting trial $(2.6 \pm 1.4$ and $0.4 \pm 0.1 \mathrm{ml} \mathrm{cm}-2 \mathrm{~min}-1$, respectively, $\mathrm{d}=2.2, \mathrm{P}<$ $0.05)$.

None of the real sweat samples contained any detectable IL-6. When comparing absorbance between sweat samples and the blank standard (TBS + $10 \%$ FCS), there was no discernible difference.

In the venous plasma samples, IL-6 was detected from all participants in both conditions. In the exercise trial, IL-6 concentration showed a tendency to increase over time, although this was not significant $(\mathrm{P}=0.269)$. During the resting trial, IL- 6 remained the same throughout the course of the trial. There were no differences in IL-6 concentration at any time point between the conditions (Figure 68b). 


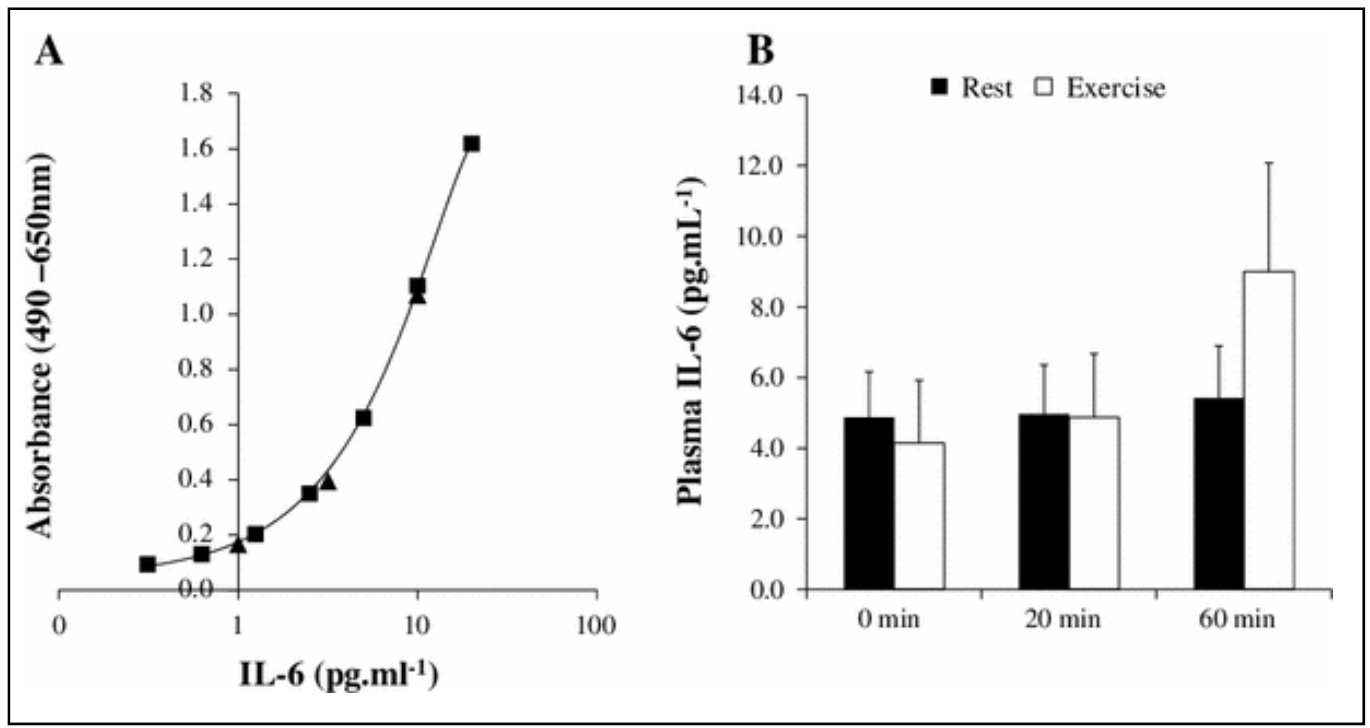

Figure 68: The detection of spiked rhIL-6 in real and artificial sweat in study 2. a) The standard curve for spiked recovery of IL-6 in artificially made sweat (filled square) and real sweat (filled triangle) indicated that there was no interference of the sweat matrix on the detection of IL-6 across a physiological range. b) Plasma IL-6 concentration did not differ between resting and exercise trials or in response to exercise.

In view of previous reports showing that IL-6 was present in sweat at concentrations comparable to that of plasma ${ }^{190,327}$, a secondary proof of concept study was conducted. Only trace amounts of IL-6 were detected in any of the sweat samples. A trace amount of IL-6 was detected following exercise in one participant, which was just below the detection range of the assay and well below the normal plasma physiological range. It was possible that the IL-6 ELISA assay was inhibited by components in the sweat. Therefore, to determine the potential interference of the sweat matrix on the IL-6 ELISA, both artificial and real sweat spiked with known concentrations of rhIL-6 was tested. When carried out in the presence of $10 \% \mathrm{FCS}$, this experiment showed that there was no difference in the detection of rhIL-6 between phosphatebuffered saline solution, artificial and real sweat, demonstrating that the ELISA protocol was not sensitive to any of the components of the sweat matrix. The assay was sensitive enough to detect IL-6 in human sweat and plasma at

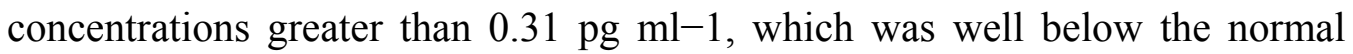
physiological plasma range of this cytokine. Therefore, it was somewhat surprising that the study was unable to replicate the findings of others that IL-6 
was present in sweat at concentrations above that of blood plasma ${ }^{190,327}$. This was especially so given that female participants were used for this part of the study to directly replicate the subject group of the previous work and that plasma IL-6 was detected at all time-points within the expected physiological range.

The difference in reported results may be due to differing ways of stimulating sweat (exercise vs. exogenous sweat gland stimulation), sweat collection methods (pouch vs. pad), the duration of collection and subject differences (healthy vs. clinical). In the studies by Marques-Deak et al. (2006) ${ }^{327}$ and Cizza et al. (2008) $)^{190}$, participants underwent a 24-h collection period wearing an absorbent pad whilst carrying out their normal daily activity. This collection method involved the sweat and its constituent parts being absorbed by a pad in contact with the skin surface. To extract the sweat, the patches were then centrifuged and the sweat was collected in a sterile tube $\mathrm{e}^{327,339}$. It was possible that the resultant sweat available for analysis contains some contamination from the absorbent pad and may give rise to false-positive or false-negative results ${ }^{337}$. In the present study, this issue was circumvented by allowing the sweat to pool during the collection period before aspiration, thus negating any potential contamination from collection pads ${ }^{340}$. Furthermore, a 20-min washout sweat collection period to allow for any epidermal or sweat gland-derived protein debris to be removed prior to collecting the main sweat sample for analysis was used. The possibility of epidermal contamination in collected sweat samples was also ruled out by Marques-Deak et al. ${ }^{327}$ who demonstrated that tape sampling of the epidermis failed to detect any cytokines at a lower detection limit of $0.1 \mathrm{pg} \mathrm{ml}^{-1} 327$.

Finally, sweat was known to contain a number of protease enzymes ${ }^{341,342}$ and protease inhibitors ${ }^{343,344}$. It was possible that during the 40-min main collection period, these enzymes may be responsible for degrading IL-6, rendering it undetectable by ELISA. However, the addition of rhIL-6 to both real and artificial sweat samples allowed for full recovery of IL-6 with no loss of signal strength. Therefore, it was unlikely that degradation was occurring during the 40-min collection period, particularly in light of the fact that the incubation 
period in the ELISA was $2 \mathrm{~h}$ in length. During this incubation period, there was a potential exposure of spiked IL-6 to the protease enzymes in real sweat. As there was close replication of the concentration of IL- 6 between both real and artificial sweat (lacking protease enzymes) it was unlikely that proteolytic degradation of IL-6 was occurring during the 40-min collection period and was not the reason this study was unable to report IL-6 in thermogenically induced sweat.

In agreement with Raiszadeh et al. ${ }^{344}$, the presented data did not support the notion that IL-6 was present in sweat in detectable levels, and thus suggest that measurement of IL-6 in sweat was not a valid or reliable measurement of systemic IL-6. 180 proteins had been identified in sweat, of which only albumin, zinc $\alpha 2$ glycoprotein, gelsolin, apolipoprotein D and clusterin 1 were present in both sweat and plasma ${ }^{344}$, indicating that future work aimed at using sweat as a potential vehicle for biomarkers or disease should focus on these proteins, as they had already been linked to a number of diseases and pathological conditions $^{345-350}$.

\subsection{CONCLUSION}

This chapter reported that the post-treatment ethanol chemical vapor deposition of GO could be utilized to increase the coverage of GO to $100 \%$ and that it could be used in as the transducer in field-effect-transistor sensors for detection of Interleukin-6 (IL-6) in the body physiological-relevant range.

Thereafter, the chapter also reported that ELISA measurement was capable of detecting IL-6 in artificial sweat at concentrations well below the normal plasma physiological concentration. Despite this, IL-6 was unsuccessful detected in sweat in of a group of five female participants either at rest in a thermally challenging environment, or following exercise. As such, it appeared that sweat was not a reliable medium for the non-invasive measurement of IL-6 in healthy or athletic populations. 


\section{CHAPTER 8: CONCLUSION}

\section{$\underline{\text { 8.1 CONCLUSION }}$}

Since an account of conclusion had been presented at the end of each chapter, this chapter assist to draw a few conclusions based on the findings of this thesis work. To recap, the goal of this thesis was to obtain an ultra-sensitive carbonbased biosensor to form the basis for point of care biomarker detection. Particular emphasis was placed on the trans-conductance, electrical homogeneity of the reduced GO transducer. The protein IL-6 was used as a marker of fatigue and overtraining in athletes and for the detection of elevated cytokines in chronic disease. The platform was also used to demonstrate the ability to detect $\mathrm{Na}^{+}$in a sweat sample which could serve as a commercially viable method for the prescribing rehydration strategies.

An improved Hummer's method that yielded the largest average GO flake size reported to date was achieved. The role and mechanism of pre-oxidation and size fractionation, was revealed. The large flakes were used in subsequent synthesis of a disposable, all-organic biosensor with integrated pump for sodium detection. The sodium sensor operates from $10 \mathrm{mM}$ to $90 \mathrm{mM}$, within the typical physiological range for sweat sodium levels.

A new carbon nano tube- reduced GO hybrid structure was then fabricated to obtain a high electrical conductivity, high optical transparency with immense degree of wrinkled surface with potential to be used in various applications.

The thesis also showed the novel growth of rGO and it mechanism of growth by the free radical condensate. The ability to obtain a complete substrate of rGO drastically reduced the both electrical resistance and electrical resistance homogeneity in biosensor transducers. The transducer was used to develop a novel biosensor for the detection of IL- 6 at the physiological relevant range of 4.7 to $300 \mathrm{pg} / \mathrm{ml}$. 
Finally, the thesis showed that IL-6 was unsuccessfully detected in sweat from participants either at rest in a thermally challenging environment, or following exercise. As such, it appears that sweat was not a simple plasma transudate as previously suggested, and was therefore not a reliable medium for the noninvasive measurement of IL-6 in healthy or athletic populations.

\subsection{FUTURE WORK}

\subsubsection{INTERLEUKIN-6 APTAMER}

Even with robust and accurate sensing platforms, there were many variable results on response of IL-6. One of the several possible explanations was the specificity and sensitivity of the ELISA assay performed in the studies ${ }^{183,351-355}$. The Interleukin-6 (IL-6) antibodies used in the immunoassay studies were obtained commercially from various companies. Although commercial companies had internal quality controls to ensure specific protein-antibody binding; the specific binding site of the antibody-protein and its robustness was unknown and of proprietary information. The users also had neither control nor knowledge over the manufacturing process and attributes could vary from batch to batch. Most importantly, antibodies were not robust enough to be used outdoors or stored for more than one day at room temperature.

An alternative to the IL-6 antibody was to utilize IL-6 aptamers. These IL-6 aptamers could be attached to the ultra-sensitive carbon-based molecular sensors to give it selectivity. Aptamers were short single-stranded nucleic acid oligomers (ssDNA or RNA) with a specific and complex three-dimensional shape such as bulges, hairpins, loops, stems, pseudoknots, triplexes, and quadruplexes $^{356}$ which could show high detection sensitivity (ranging in picomolar and nanomolar range), working range ${ }^{357}(10 \mu \mathrm{g} / 1$ to $100 \mathrm{mg} / \mathrm{l})$ and selectivity to a wide range of targets from metal ions (e.g. $\mathrm{K}+, \mathrm{Pb} 2+$ ) to a biomolecule (e.g. ATP, amino acids) to organisms (e.g. bacteria) ${ }^{358}$.

Unlike the synthesis of antibodies, which depended on induction of an animal immune system, the Systematic Evolution of Ligands by EXponential (SELEX) 
synthesis process of aptamer enabled toxic and non-immunogenic targets which were not possible to obtain by the immune system $^{359}$.

Aptamers were stable at room temperatures for long periods of time and did not aggregate easily due to lack of hydrophobic cores in aqueous solutions. They were also more robust and able to withstand or recover from $\mathrm{pH}$ and temperatures. Aptamers could be synthesized economically using well defined, highly reproducible methodologies without bacteria, cell cultures and animals $^{360}$. Also, aptamers often undergo significant conformational changes upon target binding and this could offer high detection sensitivity and selectivity in biosensors ${ }^{358}$. The disadvantages of antibodies and advantages of aptamers prompt future work on developing the IL-6 aptamer.

\subsubsection{CARBON NANOSCROLLS AND GO CURVATURE}

Carbon Nano-scrolls were promising materials for hydrogen storage ${ }^{361,362}$, supercapacitors $^{276}$, nanomechanical ${ }^{363}$ and molecular sensors ${ }^{364}$. Nano-scrolls did not have caps at the ends and it was possible to load functional molecules such as antibodies and nanoparticles along the edges or in the scrolls.

Furthermore, current only flow through the outermost shell in multi-walled CNTs, in contrast to GO nano-scrolls which could flow through the entire sheet $^{365}$. This difference resulted in low resistance of nano-scrolls.

Graphene nano-scrolls had been formed using isopropyl alcohol on mechanically exfoliated graphene sheet on $\mathrm{SiO}_{2} / \mathrm{Si}$ surface but were low yielding $^{365}$. GO nano-scrolls had been formed by facile ultrasonication with ethanol ${ }^{366}$ and functionalization of $\mathrm{GO}^{367}$. These methods were high-yielding; however, the mechanism was not yet defined. And these processes were notscalable, detrimental to the size and electrical conductivity of the GO flakes.

It was hypothesized that by selective chemical reduction on only one side of GO flake, GO could be rolled into nano-scrolls, due to the structural stress induced by different amount of oxygen moieties on both sides of the GO flake. 
Reside stress due to the inhomogeneous reduction could cause the GO to scroll. The success of this work would have tremendous impact on curvature tailoring of graphene for many applications. 


\section{REFERENCES}

1. Novoselov, K. S.; Geim, A. K.; Morozov, S. V.; Jiang, D.; Zhang, Y.; Dubonos, S. V.; Grigorieva, I. V.; Firsov, A. A., Science 2004, 306, 666-669.

2. Huang, X.; Yin, Z.; Wu, S.; Qi, X.; He, Q.; Zhang, Q.; Yan, Q.; Boey, F.; Zhang, H., Small 2011, 7, 1876-1902.

3. Castro Neto, A. H.; Guinea, F.; Peres, N. M. R.; Novoselov, K. S.; Geim, A. K., Reviews of Modern Physics 2009, 81, 109-162.

4. Schedin, F.; Geim, A. K.; Morozov, S. V.; Hill, E. W.; Blake, P.; Katsnelson, M. I.; Novoselov, K. S., Nat Mater 2007, 6, 652-655.

5. Huang, J.; Larisika, M.; Fam, W. H. D.; He, Q.; Nimmo, M. A.; Nowak, C.; Tok, I. Y. A., Nanoscale 2013, 5, 2945-2951.

6. Han, M. Y.; Özyilmaz, B.; Zhang, Y.; Kim, P., Physical Review Letters 2007, 98, 206805.

7. Ni, Z. H.; Yu, T.; Lu, Y. H.; Wang, Y. Y.; Feng, Y. P.; Shen, Z. X., ACS Nano 2008, 2, 2301-2305.

8. Ohta, T.; Bostwick, A.; Seyller, T.; Horn, K.; Rotenberg, E., Science 2006, 313, 951-954.

9. Loh, K. P.; Bao, Q.; Eda, G.; Chhowalla, M., Nature chemistry 2010, 2, 1015-1024.

10. He, Q.; Wu, S.; Gao, S.; Cao, X.; Yin, Z.; Li, H.; Chen, P.; Zhang, H., ACS Nano 2011, 5, 5038-5044.

11. He, Q.; Sudibya, H. G.; Yin, Z.; Wu, S.; Li, H.; Boey, F.; Huang, W.; Chen, P.; Zhang, H., ACS Nano 2010, 4, 3201-3208.

12. Su, C.-Y.; Xu, Y.; Zhang, W.; Zhao, J.; Liu, A.; Tang, X.; Tsai, C.-H.; Huang, Y.; Li, L.-J., ACS Nano 2010, 4, 5285-5292.

13. Su, C.-Y.; Xu, Y.; Zhang, W.; Zhao, J.; Tang, X.; Tsai, C.-H.; Li, L.-J., Chemistry of Materials 2009, 21, 5674-5680.

14. Brodie, B. C., Philosophical Transactions of the Royal Society of London 1859, 149, 249-259.

15. Hummers, W. S.; Offeman, R. E., Journal of the American Chemical Society 1958, 80, 1339-1339.

16. Li, D.; Muller, M. B.; Gilje, S.; Kaner, R. B.; Wallace, G. G., Nat Nano 2008, 3, 101-105. 
17. Wang, H.; Robinson, J. T.; Li, X.; Dai, H., Journal of the American Chemical Society 2009, 131, 9910-9911.

18. Nethravathi, C.; Rajamathi, M., Carbon 2008, 46, 1994-1998.

19. Blumen, A., CRYSTAL RESEARCH AND TECHNOLOGY 1999, 34, 937-938.

20. Kim, S.; Zhou, S.; Hu, Y.; Acik, M.; Chabal, Y. J.; Berger, C.; de Heer, W.; Bongiorno, A.; Riedo, E., Nat Mater 2012, 11, 544-549.

21. Jung, I.; Dikin, D. A.; Piner, R. D.; Ruoff, R. S., Nano Letters 2008, 8, 4283-4287.

22. Kim, S. R.; Parvez, M. K.; Chhowalla, M., Chemical Physics Letters 2009, 483, 124-127.

23. Cheng, C.; Li, D., Advanced Materials 2013, 25, 13-30.

24. Vitelli, V.; van Hecke, M., Nature 2011, 480, 325-326.

25. Mermoux, M.; Chabre, Y.; Rousseau, A., Carbon 1991, 29, 469-474.

26. Nakajima, T.; Matsuo, Y., Carbon 1994, 32, 469-475.

27. Hofmann, U.; Frenzel, A.; Csalán, E., Justus Liebigs Annalen der Chemie 1934, 510, 1-41.

28. Ruess, G., Monatshefte für Chemie 1947, 76, 381-417.

29. Scholz, W.; Boehm, H. P., Zeitschrift für anorganische und allgemeine Chemie 1969, 369, 327-340.

30. Lerf, A.; He, H.; Forster, M.; Klinowski, J., The Journal of Physical Chemistry B 1998, 102, 4477-4482.

31. Dreyer, D. R.; Park, S.; Bielawski, C. W.; Ruoff, R. S., Chemical Society Reviews 2010, 39, 228-240.

32. Stankovich, S.; Piner, R. D.; Nguyen, S. T.; Ruoff, R. S., Carbon 2006, $44,3342-3347$.

33. Loh, K. P.; Bao, Q.; Eda, G.; Chhowalla, M., Nat Chem 2010, 2, $1015-$ 1024.

34. Mao, S.; Pu, H.; Chen, J., RSC Advances 2012, 2, 2643-2662.

35. Zhou, Y.; Bao, Q.; Tang, L. A. L.; Zhong, Y.; Loh, K. P., Chemistry of Materials 2009, 21, 2950-2956.

36. Szabó, T.; Berkesi, O.; Forgó, P.; Josepovits, K.; Sanakis, Y.; Petridis, D.; Dékány, I., Chemistry of Materials 2006, 18, 2740-2749. 
37. Gao, W.; Alemany, L. B.; Ci, L.; Ajayan, P. M., Nat Chem 2009, 1, 403-408.

38. Mkhoyan, K. A.; Contryman, A. W.; Silcox, J.; Stewart, D. A.; Eda, G.; Mattevi, C.; Miller, S.; Chhowalla, M., Nano Letters 2009, 9, 1058-1063.

39. Larisika, M.; Huang, J.; Tok, A.; Knoll, W.; Nowak, C., Materials Chemistry and Physics 2012, 136, 304-308.

40. Stankovich, S.; Dikin, D. A.; Piner, R. D.; Kohlhaas, K. A.; Kleinhammes, A.; Jia, Y.; Wu, Y.; Nguyen, S. T.; Ruoff, R. S., Carbon 2007, $45,1558-1565$.

41. Robinson, J. T.; Perkins, F. K.; Snow, E. S.; Wei, Z.; Sheehan, P. E., Nano Letters 2008, 8, 3137-3140.

42. Lide, D. R., CRC Handbook of Chemistry and Physics 2004-2005: A Ready-Reference Book of Chemical and Physical Data. CRC PressI Llc: 2004.

43. Eda, G.; Chhowalla, M., Advanced Materials 2010, 22, 2392-2415.

44. Lee, D. W.; Seo, J. W., The Journal of Physical Chemistry C 2011, 115, 2705-2708.

45. Kaiser, A. B.; Gómez-Navarro, C.; Sundaram, R. S.; Burghard, M.; Kern, K., Nano Letters 2009, 9, 1787-1792.

46. Erickson, K.; Erni, R.; Lee, Z.; Alem, N.; Gannett, W.; Zettl, A., Advanced Materials 2010, 22, 4467-4472.

47. Tang, L.; Li, X.; Ji, R.; Teng, K. S.; Tai, G.; Ye, J.; Wei, C.; Lau, S. P., Journal of Materials Chemistry 2012, 22, 5676-5683.

48. Beller, G. A.; Maher, J. T.; Hartley, L. H.; Bass, D. E.; Wacker, W. E., Aviat Space Environ Med 1975, 46, 709-712.

49. Cai, J.; Ruffieux, P.; Jaafar, R.; Bieri, M.; Braun, T.; Blankenburg, S.; Muoth, M.; Seitsonen, A. P.; Saleh, M.; Feng, X.; Mullen, K.; Fasel, R., Nature 2010, 466, 470-473.

50. Chen, Y.-C.; Cao, T.; Chen, C.; Pedramrazi, Z.; Haberer, D.; de OteyzaDimas, G.; Fischer, F. R.; Louie, S. G.; Crommie, M. F., Nat Nano 2015, 10, 156-160.

51. Tour, J. M., Chemistry of Materials 2014, 26, 163-171.

52. McEvoy, N.; Nolan, H.; Ashok Kumar, N.; Hallam, T.; Duesberg, G. S., Carbon 2013, 54, 283-290.

53. Parvez, K.; Li, R.; Puniredd, S. R.; Hernandez, Y.; Hinkel, F.; Wang, S.; Feng, X.; Mullen, K., ACS Nano 2013. 
54. Ohno, Y.; Maehashi, K.; Yamashiro, Y.; Matsumoto, K., Nano Letters 2009, 9, 3318-3322.

55. Cohen-Karni, T.; Qing, Q.; Li, Q.; Fang, Y.; Lieber, C. M., Nano Letters 2010, 10, 1098-1102.

56. Sutter, P., Nat Mater 2009, 8, 171-172.

57. Lin, Y.-M.; Dimitrakopoulos, C.; Jenkins, K. A.; Farmer, D. B.; Chiu, H.-Y.; Grill, A.; Avouris, P., Science 2010, 327, 662.

58. Huang, Y.; Dong, X.; Shi, Y.; Li, C. M.; Li, L.-J.; Chen, P., Nanoscale 2010, 2, 1485-1488.

59. Dong, X. C.; Fu, D. C.; Fang, W. J.; Shi, Y. M.; Cheng, P.; Li, L. J., Small 2009, 5, 1422-1426.

60. Emtsev, K. V.; Bostwick, A.; Horn, K.; Jobst, J.; Kellogg, G. L.; Ley, L.; McChesney, J. L.; Ohta, T.; Reshanov, S. A.; Rohrl, J.; Rotenberg, E.; Schmid, A. K.; Waldmann, D.; Weber, H. B.; Seyller, T., Nat Mater 2009, 8, 203-207.

61. Kim, K. S.; Zhao, Y.; Jang, H.; Lee, S. Y.; Kim, J. M.; Kim, K. S.; Ahn, J. H.; Kim, P.; Choi, J. Y.; Hong, B. H., Nature 2009, 457, 706-710.

62. Li, X.; Cai, W.; An, J.; Kim, S.; Nah, J.; Yang, D.; Piner, R.; Velamakanni, A.; Jung, I.; Tutuc, E.; Banerjee, S. K.; Colombo, L.; Ruoff, R. S., Science 2009, 324, 1312-1314.

63. Li, X.; Cai, W.; Colombo, L.; Ruoff, R. S., Nano Letters 2009, 9, 42684272 .

64. Khan, U.; O'Neill, A.; Lotya, M.; De, S.; Coleman, J. N., Small 2010, 6, 864-871.

65. You, H.-X.; Brown, N. M. D.; Al-Assadi, K. F., Surface Science 1993, 284, 263-272.

66. Pang, S.; Tsao, H. N.; Feng, X.; Müllen, K., Advanced Materials 2009, 21, 3488-3491.

67. Butler, S. Z.; Hollen, S. M.; Cao, L.; Cui, Y.; Gupta, J. A.; Gutiérrez, H. R.; Heinz, T. F.; Hong, S. S.; Huang, J.; Ismach, A. F.; Johnston-Halperin, E.; Kuno, M.; Plashnitsa, V. V.; Robinson, R. D.; Ruoff, R. S.; Salahuddin, S.; Shan, J.; Shi, L.; Spencer, M. G.; Terrones, M.; Windl, W.; Goldberger, J. E., ACS Nano 2013.

68. Santanu, D.; Wonbong, C., Graphene Synthesis. In Graphene, CRC Press: 2011; pp 27-64.

69. Chen, G.; Weng, W.; Wu, D.; Wu, C.; Lu, J.; Wang, P.; Chen, X., Carbon 2004, 42, 753-759. 
70. Chen, G.; Wu, D.; Weng, W.; Wu, C., Carbon 2003, 41, 619-621.

71. Low, C. T. J.; Walsh, F. C.; Chakrabarti, M. H.; Hashim, M. A.; Hussain, M. A., Carbon 2013, 54, 1-21.

72. Singh, V. V.; Gupta, G.; Batra, A.; Nigam, A. K.; Boopathi, M.; Gutch, P. K.; Tripathi, B. K.; Srivastava, A.; Samuel, M.; Agarwal, G. S.; Singh, B.; Vijayaraghavan, R., Advanced Functional Materials 2012, 22, 2352-2362.

73. Nose, M.; Kinumoto, T.; Choo, H. S.; Miyazaki, K.; Abe, T.; Ogumi, Z., Fuel Cells 2009, 9, 284-290.

74. Alanyalığlu, M.; Segura, J. J.; Oró-Solè, J.; Casañ-Pastor, N., Carbon 2012, 50, 142-152.

75. Lu, J.; Yang, J.-x.; Wang, J.; Lim, A.; Wang, S.; Loh, K. P., ACS Nano 2009, 3, 2367-2375.

76. Su, C.-Y.; Lu, A.-Y.; Xu, Y.; Chen, F.-R.; Khlobystov, A. N.; Li, L.-J., ACS Nano 2011, 5, 2332-2339.

77. Liu, N.; Luo, F.; Wu, H.; Liu, Y.; Zhang, C.; Chen, J., Advanced Functional Materials 2008, 18, 1518-1525.

78. Staudenmaier, L., Berichte der deutschen chemischen Gesellschaft 1898, $31,1481-1487$.

79. Hofmann, U.; König, E., Zeitschrift für anorganische und allgemeine Chemie 1937, 234, 311-336.

80. Simon, A.; Dronskowski, R.; Krebs, B.; Hettich, B., Angewandte Chemie International Edition in English 1987, 26, 139-140.

81. Koch, K. R., Journal of Chemical Education 1982, 59, 973.

82. Poh, H. L.; Sanek, F.; Ambrosi, A.; Zhao, G.; Sofer, Z.; Pumera, M., Nanoscale 2012, 4, 3515-3522.

83. Jeong, S. Y.; Kim, S. H.; Han, J. T.; Jeong, H. J.; Jeong, S. Y.; Lee, G.W., Advanced Functional Materials 2012, 22, 3307-3314.

84. Jeong, H.-K.; Lee, Y. P.; Lahaye, R. J. W. E.; Park, M.-H.; An, K. H.; Kim, I. J.; Yang, C.-W.; Park, C. Y.; Ruoff, R. S.; Lee, Y. H., Journal of the American Chemical Society 2008, 130, 1362-1366.

85. Marcano, D. C.; Kosynkin, D. V.; Berlin, J. M.; Sinitskii, A.; Sun, Z.; Slesarev, A.; Alemany, L. B.; Lu, W.; Tour, J. M., ACS Nano 2010, 4, 48064814.

86. Kovtyukhova, N. I.; Ollivier, P. J.; Martin, B. R.; Mallouk, T. E.; Chizhik, S. A.; Buzaneva, E. V.; Gorchinskiy, A. D., Chemistry of Materials 1999, 11, 771-778. 
87. Kholmanov, I. N.; Stoller, M. D.; Edgeworth, J.; Lee, W. H.; Li, H.; Lee, J.; Barnhart, C.; Potts, J. R.; Piner, R.; Akinwande, D.; Barrick, J. E.; Ruoff, R. S., ACS Nano 2012, 6, 5157-5163.

88. Zheng, Q.; Shi, L.; Ma, P.-C.; Xue, Q.; Li, J.; Tang, Z.; Yang, J., RSC Advances 2013, 3, 4680-4691.

89. Dong, X.; Su, C.-Y.; Zhang, W.; Zhao, J.; Ling, Q.; Huang, W.; Chen, P.; Li, L.-J., Physical Chemistry Chemical Physics 2010, 12, 2164-2169.

90. Tung, V.; Allen, M.; Yang, Y.; Kaner, R., Nat Nano 2008, advanced online publication.

91. Xu, Y.; Bai, H.; Lu, G.; Li, C.; Shi, G., Journal of the American Chemical Society 2008, 130, 5856-5857.

92. Pumera, M., Chemical Society Reviews 2010, 39, 4146-4157.

93. Bagri, A.; Mattevi, C.; Acik, M.; Chabal, Y. J.; Chhowalla, M.; Shenoy, V. B., Nat Chem 2010, 2, 581-587.

94. Balberg, I., Philosophical Magazine Part B 1987, 56, 991-1003.

95. Liu, Y.; Dong, X.; Chen, P., Chemical Society Reviews 2012.

96. Mattevi, C.; Eda, G.; Agnoli, S.; Miller, S.; Mkhoyan, K. A.; Celik, O.; Mastrogiovanni, D.; Granozzi, G.; Garfunkel, E.; Chhowalla, M., Advanced Functional Materials 2009, 19, 2577-2583.

97. Zhou, M.; Wang, Y.; Zhai, Y.; Zhai, J.; Ren, W.; Wang, F.; Dong, S., Chemistry - A European Journal 2009, 15, 6116-6120.

98. Wang, Z.; Zhou, X.; Zhang, J.; Boey, F.; Zhang, H., The Journal of Physical Chemistry C 2009, 113, 14071-14075.

99. An, S. J.; Zhu, Y.; Lee, S. H.; Stoller, M. D.; Emilsson, T.; Park, S.; Velamakanni, A.; An, J.; Ruoff, R. S., The Journal of Physical Chemistry Letters 2010, 1, 1259-1263.

100. Moon, I. K.; Lee, J.; Lee, H., Chemical Communications 2011, 47, 9681-9683.

101. Dreyer, D. R.; Murali, S.; Zhu, Y.; Ruoff, R. S.; Bielawski, C. W., Journal of Materials Chemistry 2011, 21, 3443-3447.

102. Shin, H.-J.; Kim, K. K.; Benayad, A.; Yoon, S.-M.; Park, H. K.; Jung, I.-S.; Jin, M. H.; Jeong, H.-K.; Kim, J. M.; Choi, J.-Y.; Lee, Y. H., Advanced Functional Materials 2009, 19, 1987-1992.

103. Fan, X.; Peng, W.; Li, Y.; Li, X.; Wang, S.; Zhang, G.; Zhang, F., Advanced Materials 2008, 20, 4490-4493. 
104. Fan, Z.; Wang, K.; Wei, T.; Yan, J.; Song, L.; Shao, B., Carbon 2010, 48, 1686-1689.

105. Stankovich, S.; Piner, R. D.; Chen, X.; Wu, N.; Nguyen, S. T.; Ruoff, R. S., Journal of Materials Chemistry 2006, 16, 155-158.

106. Yang, D.; Velamakanni, A.; Bozoklu, G.; Park, S.; Stoller, M.; Piner, R. D.; Stankovich, S.; Jung, I.; Field, D. A.; Ventrice Jr, C. A.; Ruoff, R. S., Carbon 2009, 47, 145-152.

107. Tung, V. C.; Allen, M. J.; Yang, Y.; Kaner, R. B., Nat Nano 2009, 4, 25-29.

108. Cao, Y.-Q.; Dai, Z.; Chen, B.-H.; Liu, R., Journal of Chemical Technology \& Biotechnology 2005, 80, 834-836.

109. Gómez-Navarro, C.; Weitz, R. T.; Bittner, A. M.; Scolari, M.; Mews, A.; Burghard, M.; Kern, K., Nano Letters 2007, 7, 3499-3503.

110. Baraket, M.; Walton, S. G.; Wei, Z.; Lock, E. H.; Robinson, J. T.; Sheehan, P., Carbon 2010, 48, 3382-3390.

111. Eda, G.; Mattevi, C.; Yamaguchi, H.; Kim, H.; Chhowalla, M., The Journal of Physical Chemistry C 2009, 113, 15768-15771.

112. Kumar, N. A.; Nolan, H.; McEvoy, N.; Rezvani, E.; Doyle, R. L.; Lyons, M. E. G.; Duesberg, G. S., Journal of Materials Chemistry A 2013, 1, 44314435 .

113. Kim, M. J.; Jeong, Y.; Sohn, S.; Lee, S. Y.; Kim, Y. J.; Lee, K.; Kahng, Y. H.; Jang, J.-H., AIP Advances 2013, 3, 012117.

114. Kim, M.; Kahng, Y. H.; Kim, Y. J.; kumar, T. P.; Park, K.; Lee, K.; Jang, J.-H., AIP Advances 2013, 3, 032121-032127.

115. Pei, S.; Cheng, H.-M., Carbon 2012, 50, 3210-3228.

116. Smirnov, V. A.; Arbuzov, A. A.; Shul'ga, Y. M.; Baskakov, S. A.; Martynenko, V. M.; Muradyan, V. E.; Kresova, E. I., High Energy Chem 2011, 45, 57-61.

117. Plotnikov, V. G.; Smirnov, V. A.; Alfimov, M. V.; Shul'ga, Y. M., High Energy Chem 2011, 45, 411-415.

118. Wang, J.-N.; Shao, R.-Q.; Zhang, Y.-L.; Guo, L.; Jiang, H.-B.; Lu, D.X.; Sun, H.-B., Chemistry - An Asian Journal 2012, 7, 301-304.

119. Li, B.; Zhang, X.; Chen, P.; Li, X.; Wang, L.; Zhang, C.; Zheng, W.; Liu, Y., RSC Advances 2014, 4, 2404-2408.

120. Li, B.; Zhang, X.; Li, X.; Wang, L.; Han, R.; Liu, B.; Zheng, W.; Li, X.; Liu, Y., Chemical Communications 2010, 46, 3499-3501. 
121. Guo, L.; Jiang, H.-B.; Shao, R.-Q.; Zhang, Y.-L.; Xie, S.-Y.; Wang, J.N.; Li, X.-B.; Jiang, F.; Chen, Q.-D.; Zhang, T.; Sun, H.-B., Carbon 2012, 50, $1667-1673$.

122. Zhang, Y.; Guo, L.; Wei, S.; He, Y.; Xia, H.; Chen, Q.; Sun, H.-B.; Xiao, F.-S., Nano Today 2010, 5, 15-20.

123. Minella, M.; Demontis, M.; Sarro, M.; Sordello, F.; Calza, P.; Minero, C., J Mater Sci 2015, 50, 2399-2409.

124. Li, X.; Tang, T.; Li, M.; He, X., J Mater Sci: Mater Electron 2014, 1-6.

125. Williams, G.; Seger, B.; Kamat, P. V., ACS Nano 2008, 2, 1487-1491.

126. Shen, J.; Yan, B.; Shi, M.; Ma, H.; Li, N.; Ye, M., Journal of Materials Chemistry 2011, 21, 3415-3421.

127. Zhang, M.; Liu, S.; Yin, X. M.; Du, Z. F.; Hao, Q. Y.; Lei, D. N.; Li, Q. H.; Wang, T. H., Chemistry - An Asian Journal 2011, 6, 1151-1154.

128. Hassan, H. M. A.; Abdelsayed, V.; Khder, A. E. R. S.; AbouZeid, K. M.; Terner, J.; El-Shall, M. S.; Al-Resayes, S. I.; El-Azhary, A. A., Journal of Materials Chemistry 2009, 19, 3832-3837.

129. Chen, W.; Yan, L.; Bangal, P. R., Carbon 2010, 48, 1146-1152.

130. Zhu, Y.; Murali, S.; Stoller, M. D.; Velamakanni, A.; Piner, R. D.; Ruoff, R. S., Carbon 2010, 48, 2118-2122.

131. Liu, S.; Wu, J.; Zhou, Z.; Gao, L.; Luo, S.; Xu, X.; Wang, Z., J Mater Sci: Mater Electron 2013, 24, 1298-1302.

132. Hu, H.; Zhao, Z.; Zhou, Q.; Gogotsi, Y.; Qiu, J., Carbon 2012, 50, 3267-3273.

133. Liang, Q.; Hsie, S. A.; Wong, C. P., ChemPhysChem 2012, 13, 37003706.

134. Chen, J.; Shi, T.; Cai, T.; Xu, T.; Sun, L.; Wu, X.; Yu, D., Applied Physics Letters 2013, 102, 103107-103105.

135. Larciprete, R.; Fabris, S.; Sun, T.; Lacovig, P.; Baraldi, A.; Lizzit, S., Journal of the American Chemical Society 2011, 133, 17315-17321.

136. Li, X.; Wang, H.; Robinson, J. T.; Sanchez, H.; Diankov, G.; Dai, H., Journal of the American Chemical Society 2009, 131, 15939-15944.

137. Jung, I.; Field, D. A.; Clark, N. J.; Zhu, Y.; Yang, D.; Piner, R. D.; Stankovich, S.; Dikin, D. A.; Geisler, H.; Ventrice, C. A.; Ruoff, R. S., The Journal of Physical Chemistry C 2009, 113, 18480-18486. 
138. Schniepp, H. C.; Li, J.-L.; McAllister, M. J.; Sai, H.; Herrera-Alonso, M.; Adamson, D. H.; Prud'homme, R. K.; Car, R.; Saville, D. A.; Aksay, I. A., The Journal of Physical Chemistry B 2006, 110, 8535-8539.

139. Ferrari, A. C.; Robertson, J., Philosophical Transactions of the Royal Society of London. Series A 2004, 362, 2477-2512.

140. Buono, M. J.; Ball, K. D.; Kolkhorst, F. W., Journal of Applied Physiology 2007, 103, 990-994.

141. Shibasaki, M.; Crandall, C. G., Frontiers in bioscience (Scholar edition) 2010, 2, 685-696.

142. Uno, H., The Journal of investigative dermatology 1977, 69, 112-120.

143. Baker, L. B.; Stofan, J. R.; Hamilton, A. A.; Horswill, C. A., Journal of Applied Physiology 2009, 107, 887-895.

144. Costill, D. L., Annals of the New York Academy of Sciences 1977, 301, 160-174.

145. Sato, K.; Sato, F., The Journal of Clinical Investigation 1984, 73, 17631771.

146. Rosenstein, B. J.; Cutting, G. R., The Journal of Pediatrics 1998, 132, 589-595.

147. Montain, S. J.; Cheuvront, S. N.; Sawka, M. N., British journal of sports medicine 2006, 40, 98-105; discussion 198-105.

148. Frizzell, R.; Lang, G. H.; Lowance, D. C.; Lathan, S., JAMA 1986, 255, $772-774$.

149. Patterson, M. J.; Galloway, S. D. R.; Nimmo, M. A., Experimental Physiology 2000, 85, 869-875.

150. Buono, M. J.; Claros, R.; DeBoer, T.; Wong, J., Na+ secretion rate increases proportionally more than the $\mathrm{Na}+$ reabsorption rate with increases in sweat rate. 2008; Vol. 105, p 1044-1048.

151. Weschler, L. B., Journal of Applied Physiology 2008, 105, 1376-1377.

152. Kurihara-Bergstrom, T.; Knutson, K.; DeNoble, L.; Goates, C., Pharm Res 1990, 7, 762-766.

153. Shamsuddin, A. K. M.; Yanagimoto, S.; Kuwahara, T.; Zhang, Y.; Nomura, C.; Kondo, N., Eur J Appl Physiol 2005, 94, 292-297.

154. Inoue, Y.; Nakao, M.; Ishizashi, H.; Tsujita, J.; Araki, T., Applied Human Science 1998, 17, 219-221. 
155. Hamouti, N.; Del Coso, J.; Ortega, J.; Mora-Rodriguez, R., Eur J Appl Physiol 2011, 111, 2873-2881.

156. Kirby, C. R.; Convertino, V. A., Journal of Applied Physiology 1986, 61, 967-970.

157. Lott, M. J. E.; Galloway, S. D. R., IJSNEM 2011, 21, 8.

158. Godek, S. F.; Peduzzi, C.; Burkholder, R.; Condon, S.; Dorshimer, G.; Bartolozzi, A. R., J Athl Train 2010, 45, 364-371.

159. Mishra, A.; Greaves, R.; Massie, J., The Clinical biochemist. Reviews / Australian Association of Clinical Biochemists 2005, 26, 135-153.

160. Kirk, J. M.; Keston, M.; McIntosh, I.; al Essa, S., Annals of clinical biochemistry 1992, 29 ( Pt 2), 145-152.

161. Kartner, N.; Augustinas, O.; Jensen, T. J.; Naismith, A. L.; Riordan, J. R., Nat Genet 1992, 1, 321-327.

162. Robinson, M.; Gray, S. R.; Watson, M. S.; Kennedy, G.; Hill, A.; Belch, J. J. F.; Nimmo, M. A., Scandinavian Journal of Medicine \& Science in Sports 2010, 20, 282-290.

163. Robson-Ansley, P.; Cockburn, E.; Walshe, I.; Stevenson, E.; Nimmo, M., Exercise immunology review 2010, 16, 56-76.

164. Scheller, J.; Chalaris, A.; Schmidt-Arras, D.; Rose-John, S., Biochimica et Biophysica Acta (BBA) - Molecular Cell Research 2011, 1813, 878-888.

165. Tawara, K.; Oxford, J. T.; Jorcyk, C. L., Cancer Manag Res 2011, 3, 177-189.

166. Suwa, T.; Hogg, J. C.; English, D.; Van Eeden, S. F., American journal of physiology. Heart and circulatory physiology 2000, 279, H2954-2960.

167. Romano, M.; Sironi, M.; Toniatti, C.; Polentarutti, N.; Fruscella, P.; Ghezzi, P.; Faggioni, R.; Luini, W.; van Hinsbergh, V.; Sozzani, S.; Bussolino, F.; Poli, V.; Ciliberto, G.; Mantovani, A., Immunity 1997, 6, 315-325.

168. Malhotra, R.; Patel, V.; Vaqué, J. P.; Gutkind, J. S.; Rusling, J. F., Analytical Chemistry 2010, 82, 3118-3123.

169. Heinrich, P. C.; Behrmann, I.; Haan, S.; Hermanns, H. M.; MüllerNewen, G.; Schaper, F., Biochem. J. 2003, 374, 1-20.

170. Gray, S. R.; Ratkevicius, A.; Wackerhage, H.; Coats, P.; Nimmo, M. A., Experimental Physiology 2009, 94, 899-905.

171. Gray, S. R.; Clifford, M.; Lancaster, R.; Leggate, M.; Davies, M.; Nimmo, M. A., Cytokine 2009, 47, 98-102. 
172. Leggate, M.; Nowell, M.; Jones, S.; Nimmo, M., Cell Stress and Chaperones 2010, 15, 827-833.

173. Fischer, C. P., Exercise immunology review 2006, 12, 6-33.

174. Pedersen, B. K.; Toft, A. D., British journal of sports medicine 2000, 34, 246-251.

175. Sprenger, H.; Jacobs, C.; Nain, M.; Gressner, A. M.; Prinz, H.; Wesemann, W.; Gemsa, D., Clinical Immunology and Immunopathology 1992, 63, 188-195.

176. Robson, P., Sports Med 2003, 33, 771-781.

177. Spath-Schwalbe, E.; Hansen, K.; Schmidt, F.; Schrezenmeier, H.; Marshall, L.; Burger, K.; Fehm, H. L.; Born, J., The Journal of clinical endocrinology and metabolism 1998, 83, 1573-1579.

178. Robson-Ansley, P. J.; de Milander, L.; Collins, M.; Noakes, T. D., Canadian journal of applied physiology $=$ Revue canadienne de physiologie appliquee 2004, 29, 411-418.

179. Kreher, J. B.; Schwartz, J. B., Sports health 2012, 4, 128-138.

180. Pedersen, B. K., Medicine and science in sports and exercise 2012, 44, 392-396.

181. Helge, J. W.; Stallknecht, B.; Pedersen, B. K.; Galbo, H.; Kiens, B.; Richter, E. A., The Journal of physiology 2003, 546, 299-305.

182. Bruunsgaard, H.; Galbo, H.; Halkjaer-Kristensen, J.; Johansen, T. L.; MacLean, D. A.; Pedersen, B. K., The Journal of physiology 1997, 499 ( Pt 3), 833-841.

183. Pedersen, B. K., Immunol Cell Biol 2000, 78, 532-535.

184. Houssiau, F. A.; Devogelaer, J.-P.; Damme, J. V.; Deuxchaisnes, C. N. D.; Snick, J. V., Arthritis \& Rheumatism 1988, 31, 784-788.

185. Grossman, R. M.; Krueger, J.; Yourish, D.; Granelli-Piperno, A.; Murphy, D. P.; May, L. T.; Kupper, T. S.; Sehgal, P. B.; Gottlieb, A. B., Proceedings of the National Academy of Sciences of the United States of America 1989, 86, 6367-6371.

186. Singh, P.; Goyal, A., SpringerPlus 2013, 2, 686.

187. Hashizume, M.; Mihara, M., Arthritis 2011, 2011.

188. Minetto, M.; Rainoldi, A.; Gazzoni, M.; Terzolo, M.; Borrione, P.; Termine, A.; Saba, L.; Dovio, A.; Angeli, A.; Paccotti, P., Eur J Appl Physiol 2005, 93, 679-686. 
189. Marques-Deak, A.; Cizza, G.; Eskandari, F.; Torvik, S.; Christie, I. C.; Sternberg, E. M.; Phillips, T. M., Journal of immunological methods 2006, 315, 99-109.

190. Cizza, G.; Marques, A. H.; Eskandari, F.; Christie, I. C.; Torvik, S.; Silverman, M. N.; Phillips, T. M.; Sternberg, E. M., Biological psychiatry 2008, 64, 907-911.

191. Raiszadeh, M. M.; Ross, M. M.; Russo, P. S.; Schaepper, M. A.; Zhou, W.; Deng, J.; Ng, D.; Dickson, A.; Dickson, C.; Strom, M.; Osorio, C.; Soeprono, T.; Wulfkuhle, J. D.; Petricoin, E. F.; Liotta, L. A.; Kirsch, W. M., Journal of proteome research 2012, 11, 2127-2139.

192. Teng, X.; Zhu, Y.; Wei, W.; Wang, S.; Huang, J.; Naccache, R.; Hu, W.; Tok, A. I. Y.; Han, Y.; Zhang, Q.; Fan, Q.; Huang, W.; Capobianco, J. A.; Huang, L., Journal of the American Chemical Society 2012, 134, 8340-8343.

193. Fam, D. W. H.; Tok, A. I. Y.; Palaniappan, A.; Nopphawan, P.; Lohani, A.; Mhaisalkar, S. G., Sensors and Actuators B: Chemical 2009, 138, 189-192.

194. Fam, D. W. H.; Tok, A. I. Y., Journal of Colloid and Interface Science 2009, 338, 266-269.

195. Shen, H.; Zhang, L. M.; Liu, M.; Zhang, Z. J., Theranostics 2012, 2, 283-294.

196. Kim, Y. K.; Kim, M. H.; Min, D. H., Chem Commun (Camb) 2011, 47, 3195-3197.

197. Jiang, H. J., Small 2011, 7, 2413-2427.

198. Pumera, M., Materials Today 2011, 14, 308-315.

199. Jang, H.; Kim, Y. K.; Kwon, H. M.; Yeo, W. S.; Kim, D. E.; Min, D. H., Angew Chem Int Edit 2010, 49, 5703-5707.

200. Lu, C. H.; Zhu, C. L.; Li, J.; Liu, J. J.; Chen, X.; Yang, H. H., Chem Commun (Camb) 2010, 46, 3116-3118.

201. Balapanuru, J.; Yang, J.-X.; Xiao, S.; Bao, Q.; Jahan, M.; Polavarapu, L.; Wei, J.; Xu, Q.-H.; Loh, K. P., Angewandte Chemie 2010, 122, 6699-6703.

202. Liu, F.; Choi, J. Y.; Seo, T. S., Biosensors and Bioelectronics 2010, 25, 2361-2365.

203. Kuila, T.; Bose, S.; Khanra, P.; Mishra, A. K.; Kim, N. H.; Lee, J. H., Biosensors \& Bioelectronics 2011, 26, 4637-4648.

204. Stine, R.; Robinson, J. T.; Sheehan, P. E.; Tamanaha, C. R., Advanced Materials 2010, 22, 5297-5300. 
205. Tao, Y.; Lin, Y.; Huang, Z.; Ren, J.; Qu, X., Analyst 2012, 137, 25882592.

206. Jung, J. H.; Cheon, D. S.; Liu, F.; Lee, K. B.; Seo, T. S., Angew Chem Int Edit 2010, 49, 5708-5711.

207. Chang, H.; Tang, L.; Wang, Y.; Jiang, J.; Li, J., Analytical Chemistry 2010, 82, 2341-2346.

208. Palecek, E.; Fojta, M., Analytical Chemistry 2001, 73, 74a-83a.

209. Zhou, M.; Zhai, Y.; Dong, S., Analytical Chemistry 2009, 81, 56035613.

210. Ambrosi, A.; Sasaki, T.; Pumera, M., Chem Asian J 2010, 5, 266-271.

211. Kim, Y. R.; Bong, S.; Kang, Y. J.; Yang, Y.; Mahajan, R. K.; Kim, J. S.; Kim, H., Biosensors \& Bioelectronics 2010, 25, 2366-2369.

212. Lu, C.-H.; Yang, H.-H.; Zhu, C.-L.; Chen, X.; Chen, G.-N., Angewandte Chemie International Edition 2009, 48, 4785-4787.

213. Chang, H. X.; Tang, L. H.; Wang, Y.; Jiang, J. H.; Li, J. H., Analytical Chemistry 2010, 82, 2341-2346.

214. Balapanuru, J.; Yang, J. X.; Xiao, S.; Bao, Q. L.; Jahan, M.; Polavarapu, L.; Wei, J.; Xu, Q. H.; Loh, K. P., Angew Chem Int Edit 2010, 49, 6549-6553.

215. He, S. J.; Song, B.; Li, D.; Zhu, C. F.; Qi, W. P.; Wen, Y. Q.; Wang, L. H.; Song, S. P.; Fang, H. P.; Fan, C. H., Advanced Functional Materials 2010, 20, 453-459.

216. Choi, B. G.; Park, H.; Park, T. J.; Yang, M. H.; Kim, J. S.; Jang, S.-Y.; Heo, N. S.; Lee, S. Y.; Kong, J.; Hong, W. H., ACS Nano 2010, 4, 2910-2918.

217. Song, Y. J.; Qu, K. G.; Zhao, C.; Ren, J. S.; Qu, X. G., Advanced Materials 2010, 22, 2206-+.

218. Cong, H. P.; He, J. J.; Lu, Y.; Yu, S. H., Small 2010, 6, 169-173.

219. Liu, S.; Guo, X. F., Npg Asia Mater 2012, 4, 1-10.

220. Huang, J.; Harvey, J.; Fam, W. H. D.; Nimmo, M. A.; Tok, I. Y. A., Procedia Engineering 2013.

221. Huang, J.; Harvey, J.; Chen, H.; Nimmo, M. A.; Tok, I. Y. A. In Fully Organic Graphene Oxide-based Sensor with Integrated Pump for Sodium Detection, Conference Proceeding of icSports, Vilamoura, Portugal, Vilamoura, Portugal, 2013; pp 83-88.

222. Mohanty, N.; Berry, V., Nano Lett 2008, 8, 4469-4476. 
223. Agarwal, S.; Zhou, X. Z.; Ye, F.; He, Q. Y.; Chen, G. C. K.; Soo, J.; Boey, F.; Zhang, H.; Chen, P., Langmuir 2010, 26, 2244-2247.

224. Sudibya, H. G.; He, Q. Y.; Zhang, H.; Chen, P., ACS Nano 2011, 5, 1990-1994.

225. Mao, S.; Lu, G. H.; Yu, K. H.; Bo, Z.; Chen, J. H., Advanced Materials 2010, 22, 3521-+.

226. Holford, T. R. J.; Davis, F.; Higson, S. P. J., Biosensors \& Bioelectronics 2012, 34, 12-24.

227. Rusling, J. F.; Sotzing, G.; Papadimitrakopoulosa, F., Bioelectrochemistry 2009, 76, 189-194.

228. Mao, S.; Lu, G.; Yu, K.; Bo, Z.; Chen, J., Advanced Materials 2010, 22, 3521-3526.

229. Wang, Y.; Li, Y.; Tang, L.; Lu, J.; Li, J., Electrochemistry Communications 2009, 11, 889-892.

230. Schazmann, B.; Morris, D.; Slater, C.; Beirne, S.; Fay, C.; Reuveny, R.; Moyna, N.; Diamond, D., Analytical Methods 2010, 2, 342-348.

231. Coyle, S.; King-Tong, L.; Moyna, N.; O'Gorman, D.; Diamond, D.; Di Francesco, F.; Costanzo, D.; Salvo, P.; Trivella, M. G.; De Rossi, D. E.; Taccini, N.; Paradiso, R.; Porchet, J. A.; Ridolfi, A.; Luprano, J.; Chuzel, C.; Lanier, T.; Revol-Cavalier, F.; Schoumacker, S.; Mourier, V.; Chartier, I.; Convert, R.; DeMoncuit, H.; Bini, C., Information Technology in Biomedicine, IEEE Transactions on 2010, 14, 364-370.

232. Li, D.; Muller, M. B.; Gilje, S.; Kaner, R. B.; Wallace, G. G., Nat. Nanotechnol. 2008, 3, 101-105.

233. Yoo, E.; Kim, J.; Hosono, E.; Zhou, H.; Kudo, T.; Honma, I., Nano Lett. 2008, 8, 2277-2282.

234. Das, A.; Pisana, S.; Chakraborty, B.; Piscanec, S.; Saha, S. K.; Waghmare, U. V.; Novoselov, K. S.; Krishnamurthy, H. R.; Geim, A. K.; Ferrari, A. C., Nat. Nanotechnol. 2008, 3, 210.

235. Sui, Y.; Appenzeller, J., Nano Letters 2009, 9, 2973-2977.

236. Ang, P. K.; Chen, W.; Wee, A. T. S.; Loh, K. P., Journal of the American Chemical Society 2008, 130, 14392-14393.

237. Geim, A. K.; Novoselov, K. S., Nat Mater 2007, 6, 183-191.

238. Rao, C. N. R.; Sood, A. K.; Subrahmanyam, K. S.; Govindaraj, A., Angewandte Chemie International Edition 2009, 48, 7752-7777. 
239. Ishigami, M.; Chen, J. H.; Cullen, W. G.; Fuhrer, M. S.; Williams, E. D., Nano Letters 2007, 7, 1643-1648.

240. Luo, Z.; Lu, Y.; Somers, L. A.; Johnson, A. T. C., J. Am. Chem. Soc. 2009, 131, 898-899.

241. Hou, S.; Su, S.; Kasner, M. L.; Shah, P.; Patel, K.; Madarang, C. J., Chemical Physics Letters 2010, 501, 68-74.

242. Luo, B.; Yan, X.; Xu, S.; Xue, Q., Electrochimica Acta 2012, 59, 429434.

243. Szabó, T.; Berkesi, O.; Dékány, I., Carbon 2005, 43, 3186-3189.

244. Park, S.; Ruoff, R. S., Nat Nano 2009, 4, 217-224.

245. Eda, G.; Fanchini, G.; Chhowalla, M., Nat Nano 2008, 3, 270-274.

246. Hernandez, Y.; Nicolosi, V.; Lotya, M.; Blighe, F. M.; Sun, Z.; De, S.; McGovern, I. T.; Holland, B.; Byrne, M.; Gun'Ko, Y. K.; Boland, J. J.; Niraj, P.; Duesberg, G.; Krishnamurthy, S.; Goodhue, R.; Hutchison, J.; Scardaci, V.; Ferrari, A. C.; Coleman, J. N., Nat Nanotechnol 2008, 3, 563-568.

247. Gao, L.; Guest, J. R.; Guisinger, N. P., Nano Letters 2010, 10, 35123516 .

248. Gilje, S.; Han, S.; Wang, M.; Wang, K. L.; Kaner, R. B., Nano Letters 2007, 7, 3394-3398.

249. Stankovich, S.; Piner, R. D.; Chen, X.; Wu, N.; Nguyen, S. T.; Ruoff, R. S., J. Mater. Chem. 2006, 16, 155-158.

250. Becerril, H. A.; Mao, J.; Liu, Z.; Stoltenberg, R. M.; Bao, Z.; Chen, Y., ACS Nano 2008, 2, 463.

251. Cote, L. J.; Kim, F.; Huang, J., J. Am. Chem. Soc. 2009, 131, 1043-1049.

252. Wu, J.; Becerril, H. A.; Bao, Z.; Liu, Z.; Chen, Y.; Peumans, P., Appl. Phys. Lett. 2008, 92, 263302-263301.

253. GIA Biosensors in Medical Diagnostics; CA, 2012; 403.

254. Huang, J.; Harvey, J.; Fam, W. H. D.; Nimmo, M. A.; Tok, I. Y. A., Procedia Engineering 2013, Submitted.

255. Huang, J.; Larisika, M.; Fam, W. H. D.; He, Q.; Nimmo, M. A.; Nowak, C.; Tok, A. I. Y., Nanoscale 2013, 5, 2945-2951.

256. Huang, J.; Larisika, M.; Nowak, C.; Tok, I. Y. A., New Methods in Aqueous Graphene (Graphene Oxide) Synthesis for Biosensor Devices. Taylor \& Francis Group: Oxford, UK, 2015; Vol. 1. 
257. Baker, L. B. S. J. R. H. A. A. H. C. A., Journal of Applied Physiology 2009, 107, 887-895.

258. Shateri-Khalilabad, M.; Yazdanshenas, M. E., Carbohydrate Polymers 2013, 96, 190-195.

259. Reches, M.; Mirica, K. A.; Dasgupta, R.; Dickey, M. D.; Butte, M. J.; Whitesides, G. M., ACS Applied Materials \& Interfaces 2010, 2, 1722-1728.

260. Li, X.; Tian, J.; Shen, W., ACS Applied Materials \& Interfaces 2009, 2, $1-6$.

261. Huang, J.; Harvey, J.; Chen, H.; Fam, W. H. D.; Nimmo, M. A.; Tok, I. Y. A., Chinese Physics B 2013, 23, 088104.

262. Huang, J.; Harvey, J.; Fam, W. H. D.; Nimmo, M. A.; Tok, I. Y. A., Procedia Engineering 2013, 60, 195-200.

263. Yu, G.; Hu, L.; Liu, N.; Wang, H.; Vosgueritchian, M.; Yang, Y.; Cui, Y.; Bao, Z., Nano Letters 2011, 11, 4438-4442.

264. King, P. J.; Khan, U.; Lotya, M.; De, S.; Coleman, J. N., ACS Nano 2010, 4, 4238-4246.

265. Shen, L.; Zhang, X.; Li, H.; Yuan, C.; Cao, G., The Journal of Physical Chemistry Letters 2011, 2, 3096-3101.

266. Wang, Y.; Wu, Y.; Huang, Y.; Zhang, F.; Yang, X.; Ma, Y.; Chen, Y., The Journal of Physical Chemistry C 2011, 115, 23192-23197.

267. Becerril, H. A.; Mao, J.; Liu, Z.; Stoltenberg, R. M.; Bao, Z.; Chen, Y., ACS Nano 2008, 2, 463-470.

268. Yu, D.; Dai, L., The Journal of Physical Chemistry Letters 2009, 1, 467470.

269. Kim, Y.-K.; Min, D.-H., Langmuir 2009, 25, 11302-11306.

270. Hong, T.-K.; Lee, D. W.; Choi, H. J.; Shin, H. S.; Kim, B.-S., ACS Nano 2010, 4, 3861-3868.

271. Fan, Z.; Yan, J.; Zhi, L.; Zhang, Q.; Wei, T.; Feng, J.; Zhang, M.; Qian, W.; Wei, F., Advanced Materials 2010, 22, 3723-3728.

272. Ambrosi, A.; Pumera, M., The Journal of Physical Chemistry C 2011, $115,25281-25284$.

273. Qian, Z.; Shaojun, Y.; Jing, Z.; Ling, Z.; Pingli, K.; Jinghong, L.; Jingwei, X.; Hua, Z.; Xi-Ming, S., Nanotechnology 2011, 22, 494010.

274. Yu, A.; Ramesh, P.; Sun, X.; Bekyarova, E.; Itkis, M. E.; Haddon, R. C., Advanced Materials 2008, 20, 4740-4744. 
275. Tung, V. C.; Chen, L.-M.; Allen, M. J.; Wassei, J. K.; Nelson, K.; Kaner, R. B.; Yang, Y., Nano Letters 2009, 9, 1949-1955.

276. Dong, X.; Xing, G.; Chan-Park, M. B.; Shi, W.; Xiao, N.; Wang, J.; Yan, Q.; Sum, T. C.; Huang, W.; Chen, P., Carbon 2011, 49, 5071-5078.

277. Foley, M. Utilizing nanoscale material as dispersants, surfactants or stabilizing molecules, methods of making the same and products produced therefrom. 2011.

278. Fernández-Merino, M. J.; Guardia, L.; Paredes, J. I.; Villar-Rodil, S.; Solís-Fernández, P.; Martínez-Alonso, A.; Tascón, J. M. D., The Journal of Physical Chemistry C 2010, 114, 6426-6432.

279. Liu, Y.; Tang, J.; Wang, R.; Lu, H.; Li, L.; Kong, Y.; Qi, K.; Xin, J. H., Journal of Materials Chemistry 2007, 17, 1071-1078.

280. Wu, Q.; Wu, Y.; Hao, Y.; Geng, J.; Charlton, M.; Chen, S.; Ren, Y.; Ji, H.; Li, H.; Boukhvalov, D. W.; Piner, R. D.; Bielawski, C. W.; Ruoff, R. S., Chemical Communications 2013, 49, 677-679.

281. Zhu, W.; Low, T.; Perebeinos, V.; Bol, A. A.; Zhu, Y.; Yan, H.; Tersoff, J.; Avouris, P., Nano Letters 2012, 12, 3431-3436.

282. Tang, L. A. L.; Lee, W. C.; Shi, H.; Wong, E. Y. L.; Sadovoy, A.; Gorelik, S.; Hobley, J.; Lim, C. T.; Loh, K. P., Small 2012, 8, 423-431.

283. Yang, N.; Zhai, J.; Wang, D.; Chen, Y.; Jiang, L., ACS Nano 2010, 4, 887-894.

284. Pan, Z.; Liu, N.; Fu, L.; Liu, Z., Journal of the American Chemical Society 2011, 133, 17578-17581.

285. Krishnan, D.; Raidongia, K.; Shao, J.; Huang, J., ACS Nano 2013, 8, 449-457.

286. Wang, J.; Jiang, L.; Du, H.; Mason, G. F., Journal of Neuroscience Methods 2012, 208, 79-85.

287. Huang, J.; Fam, D.; He, Q.; Chen, H.; Zhan, D.; Faulkner, S. H.; Nimmo, M. A.; Yoong Tok, A. I., Journal of Materials Chemistry C 2014, 2, 109-114.

288. Li, J.; He, Y.; Han, Y.; Liu, K.; Wang, J.; Li, Q.; Fan, S.; Jiang, K., Nano Letters 2012, 12, 4095-4101.

289. Novoselov, K. S.; Geim, A. K.; Morozov, S. V.; Jiang, D.; Katsnelson, M. I.; Grigorieva, I. V.; Dubonos, S. V.; Firsov, A. A., Nature 2005, 438, $197-$ 200 .

290. Wilson, N. R.; Pandey, P. A.; Beanland, R.; Young, R. J.; Kinloch, I. A.; Gong, L.; Liu, Z.; Suenaga, K.; Rourke, J. P.; York, S. J.; Sloan, J., ACS Nano 2009, 3, 2547-2556. 
291. Wang, G.; Yang, J.; Park, J.; Gou, X.; Wang, B.; Liu, H.; Yao, J., The Journal of Physical Chemistry C 2008, 112, 8192-8195.

292. Zhang, Y.; Li, Z.; Kim, P.; Zhang, L.; Zhou, C., ACS Nano 2011, 6, 126-132.

293. Yuan, G. D.; Zhang, W. J.; Yang, Y.; Tang, Y. B.; Li, Y. Q.; Wang, J. X.; Meng, X. M.; He, Z. B.; Wu, C. M. L.; Bello, I.; Lee, C. S.; Lee, S. T., Chemical Physics Letters 2009, 467, 361-364.

294. Reilly, P. T. A.; Whitten, W. B., Carbon 2006, 44, 1653-1660.

295. Ferrari, A. C., Solid State Communications 2007, 143, 47-57.

296. Yao, Y.; Feng, C.; Zhang, J.; Liu, Z., Nano Letters 2009, 9, 1673-1677.

297. Dutta, S.; Pati, S. K., Journal of Materials Chemistry 2010, 20, 82078223.

298. Baumgartner, J.; Dey, A.; Bomans, P. H. H.; Le Coadou, C.; Fratzl, P.; Sommerdijk, N. A. J. M.; Faivre, D., Nat Mater 2013, 12, 310-314.

299. Okada, K.; Kanda, H.; Komatsu, S.; Matsumoto, S., Journal of Applied Physics 2000, 88, 1674-1678.

300. Ferrari, A. C.; Robertson, J., Physical Review B 2001, 64, 075414.

301. Calizo, I.; Bejenari, I.; Rahman, M.; Liu, G.; Balandin, A. A., Journal of Applied Physics 2009, 106, 043509-043505.

302. Ferrari, A. C., MRS Online Proceedings Library 2001, 675, null-null.

303. Tuinstra, F.; Koenig, J. L., The Journal of Chemical Physics 1970, 53, 1126-1130.

304. Haghighatpanah, S.; Börjesson, A.; Amara, H.; Bichara, C.; Bolton, K., Physical Review B 2012, 85, 205448.

305. Gavillet, J.; Loiseau, A.; Ducastelle, F.; Thair, S.; Bernier, P.; Stéphan, O.; Thibault, J.; Charlier, J. C., Carbon 2002, 40, 1649-1663.

306. Faulkner, S.; Spilsbury, K.; Harvey, J.; Jackson, A.; Huang, J.; Platt, M.; Tok, A.; Nimmo, M., Eur J Appl Physiol 2014, 114, 1207-1216.

307. Gray, S. R.; Robinson, M.; Nimmo, M. A., Cell stress \& chaperones 2008, 13, 247-251.

308. Heller, I.; Janssens, A. M.; Männik, J.; Minot, E. D.; Lemay, S. G.; Dekker, C., Nano Letters 2007, 8, 591-595.

309. Zor, E.; Hatay Patir, I.; Bingol, H.; Ersoz, M., Biosensors and Bioelectronics 2013, 42, 321-325. 
310. Hernández, R.; Vallés, C.; Benito, A. M.; Maser, W. K.; Xavier Rius, F.; Riu, J., Biosensors and Bioelectronics 2014, 54, 553-557.

311. Jang, H. D.; Kim, S. K.; Chang, H.; Roh, K.-M.; Choi, J.-W.; Huang, J., Biosensors and Bioelectronics 2012, 38, 184-188.

312. Zhang, Z.; Luo, L.; Chen, G.; Ding, Y.; Deng, D.; Fan, C., Biosensors and Bioelectronics 2014, 60, 161-166.

313. Kim, D.-J.; Sohn, I. Y.; Jung, J.-H.; Yoon, O. J.; Lee, N. E.; Park, J.-S., Biosensors and Bioelectronics 2013, 41, 621-626.

314. Cai, W.; Piner, R. D.; Stadermann, F. J.; Park, S.; Shaibat, M. A.; Ishii, Y.; Yang, D.; Velamakanni, A.; An, S. J.; Stoller, M.; An, J.; Chen, D.; Ruoff, R. S., Science 2008, 321, 1815-1817.

315. Boukhvalov, D. W.; Katsnelson, M. I., Journal of the American Chemical Society 2008, 130, 10697-10701.

316. Uygun, Z. O.; Sezgintürk, M. K., Biosensors for Cancer Biomarkers, Book title: Biosensors - Emerging Materials and Applications. InTech: 2011.

317. Lee, J. S.; Joung, H.-A.; Kim, M.-G.; Park, C. B., ACS Nano 2012, 6, 2978-2983.

318. Tu, W.; Wang, W.; Lei, J.; Deng, S.; Ju, H., Chemical Communications 2012, 48, 6535-6537.

319. Knopp, J.; Weber, G., Journal of Biological Chemistry 1967, 242, 13531354

320. Sohn, I.-Y.; Kim, D.-J.; Jung, J.-H.; Yoon, O. J.; Nguyen Thanh, T.; Tran Quang, T.; Lee, N.-E., Biosensors and Bioelectronics 2013, 45, 70-76.

321. Chen, F.; Xia, J.; Tao, N., Nano Letters 2009, 9, 1621-1625.

322. Chen, F.; Qing, Q.; Xia, J.; Li, J.; Tao, N., Journal of the American Chemical Society 2009, 131, 9908-9909.

323. Kim, J. P.; Lee, B. Y.; Lee, J.; Hong, S.; Sim, S. J., Biosensors and Bioelectronics 2009, 24, 3372-3378.

324. Abe, M.; Murata, K.; Kojima, A.; Ifuku, Y.; Shimizu, M.; Ataka, T.; Matsumoto, K., The Journal of Physical Chemistry C 2007, 111, 8667-8670.

325. Yang, T.; Wang, S.; Jin, H.; Bao, W.; Huang, S.; Wang, J., Sensors and Actuators B: Chemical 2013, 178, 310-315.

326. Ostrowski, K.; Rohde, T.; Asp, S.; Schjerling, P.; Pedersen, B. K., The Journal of physiology 1999, 515 ( Pt 1), 287-291. 
327. Marques-Deak, A.; Cizza, G.; Eskandari, F.; Torvik, S.; Christie, I. C.; Sternberg, E. M.; Phillips, T. M., Journal of immunological methods 2006, 315, 99-109.

328. Chou, T.-H.; Chuang, C.-Y.; Wu, C.-M., Cytokine 2010, 51, 107-111.

329. Pepper, J.; Noring, R.; Klempner, M.; Cunningham, B.; Petrovich, A.; Bousquet, R.; Clapp, C.; Brady, J.; Hugh, B., Sensors and Actuators B: Chemical 2003, 96, 565-575.

330. Kapoor, R.; Wang, C.-W., Biosensors and Bioelectronics 2009, 24, 2696-2701.

331. Li, T.; Yang, M., Sensors and Actuators B: Chemical 2011, 158, 361365.

332. Shi, J.-J.; He, T.-T.; Jiang, F.; Abdel-Halim, E. S.; Zhu, J.-J., Biosensors and Bioelectronics 2014, 55, 51-56.

333. Hun, X.; Zhang, Z., Biosensors and Bioelectronics 2007, 22, 2743-2748.

334. Cox, A. J.; Pyne, D. B.; Gleson, M.; Callister, R., Eur J Appl Physiol 2008, 103, 477-479.

335. Minetto, M. A.; Gazzoni, M.; Lanfranco, F.; Baldi, M.; Saba, L.; Pedrola, R.; Komi, P. V.; Rainoldi, A., Eur J Appl Physiol 2007, 101, 249-256.

336. Dai, X.; Okazaki, H.; Hanakawa, Y.; Murakami, M.; Tohyama, M.; Shirakata, Y.; Sayama, K., PloS one 2013, 8, e67666.

337. Brisson, G. R.; Boisvert, P.; Peronnet, F.; Perrault, H.; Boisvert, D.; Lafond, J. S., European journal of applied physiology and occupational physiology 1991, 63, 269-272.

338. Midander, K.; Pan, J.; Wallinder, I. O.; Heim, K.; Leygraf, C., Contact Dermatitis 2007, 56, 325-330.

339. Sarno, M.; Sarno, L.; Baylink, D.; Drinkwater, B.; Farley, S.; Kleerekoper, M.; Lang, R.; Lappe, J.; Licata, A.; McClung, M.; Miller, P.; Nattrass, S.; Recker, R.; Schwartz, E. N.; Tucci, J. R.; Wolf, S.; Powell, H.; Tjersland, G.; Warnick, G. R., Clinical chemistry and laboratory medicine : CCLM / FESCC 2001, 39, 223-228.

340. Boysen, T. C.; Yanagawa, S.; Sato, F.; Sato, K., Journal of applied physiology: respiratory, environmental and exercise physiology 1984, 56, 1302-1307.

341. Baechle, D.; Flad, T.; Cansier, A.; Steffen, H.; Schittek, B.; Tolson, J.; Herrmann, T.; Dihazi, H.; Beck, A.; Mueller, G. A.; Mueller, M.; Stevanovic, S.; Garbe, C.; Mueller, C. A.; Kalbacher, H., The Journal of biological chemistry 2006, 281, 5406-5415. 
342. Horie, N.; Yokozeki, H.; Sato, K., The American journal of physiology 1986, 250, R691-698.

343. Park, J. H.; Park, G. T.; Cho, I. H.; Sim, S. M.; Yang, J. M.; Lee, D. Y., Exp Dermatol 2011, 20, 369-371.

344. Raiszadeh, M. M.; Ross, M. M.; Russo, P. S.; Schaepper, M. A.; Zhou, W.; Deng, J.; Ng, D.; Dickson, A.; Dickson, C.; Strom, M.; Osorio, C.; Soeprono, T.; Wulfkuhle, J. D.; Petricoin, E. F.; Liotta, L. A.; Kirsch, W. M., Journal of proteome research 2012, 11, 2127-2139.

345. Ceperuelo-Mallafre, V.; Naf, S.; Escote, X.; Caubet, E.; Gomez, J. M.; Miranda, M.; Chacon, M. R.; Gonzalez-Clemente, J. M.; Gallart, L.; Gutierrez, C.; Vendrell, J., The Journal of clinical endocrinology and metabolism 2009, 94, 5062-5069.

346. Dubois, V.; Delort, L.; Mishellany, F.; Jarde, T.; Billard, H.; Lequeux, C.; Damour, O.; Penault-Llorca, F.; Vasson, M. P.; Caldefie-Chezet, F., Anticancer research 2010, 30, 2919-2925.

347. Hassan, M. I.; Waheed, A.; Yadav, S.; Singh, T. P.; Ahmad, F., Molecular cancer research : MCR 2008, 6, 892-906.

348. Mracek, T.; Stephens, N. A.; Gao, D.; Bao, Y.; Ross, J. A.; Ryden, M.; Arner, P.; Trayhurn, P.; Fearon, K. C.; Bing, C., British journal of cancer 2011, 104, 441-447.

349. Ryden, M.; Agustsson, T.; Andersson, J.; Bolinder, J.; Toft, E.; Arner, P., Journal of internal medicine 2012, 271, 414-420.

350. Yang, M.; Liu, R.; Li, S.; Luo, Y.; Zhang, Y.; Zhang, L.; Liu, D.; Wang, Y.; Xiong, Z.; Boden, G.; Chen, S.; Li, L.; Yang, G., Diabetes care 2013, 36, 1074-1082.

351. Smith, C.; Emsley, H.; Gavin, C.; Georgiou, R.; Vail, A.; Barberan, E.; del Zoppo, G.; Hallenbeck, J.; Rothwell, N.; Hopkins, S.; Tyrrell, P., BMC Neurology 2004, 4, 2.

352. Hwang, S. Y.; Kim, J. Y.; Kim, K. W.; Park, M. K.; Moon, Y.; Kim, W. U.; Kim, H. Y., Arthritis research \& therapy 2004, 6, R120-128.

353. Bonfield, T. L.; Colton, E.; Marchant, R. E.; Anderson, J. M., J Biomed Mater Res 1992, 26, 837-850.

354. Helle, M.; Boeije, L.; de Groot, E.; de Vos, A.; Aarden, L., Journal of immunological methods 1991, 138, 47-56.

355. Park, H. S.; Park, J. Y.; Yu, R., Diabetes Research and Clinical Practice 2005, 69, 29-35.

356. Stoltenburg, R.; Reinemann, C.; Strehlitz, B., Biomolecular engineering 2007, 24, 381-403. 
357. Pultar, J.; Sauer, U.; Domnanich, P.; Preininger, C., Biosensors and Bioelectronics 2009, 24, 1456-1461.

358. Song, S.; Wang, L.; Li, J.; Fan, C.; Zhao, J., TrAC Trends in Analytical Chemistry 2008, 27, 108-117.

359. Jayasena, S. D., Clinical chemistry 1999, 45, 1628-1650.

360. Jayasena, S. D., Clinical chemistry 1999, 45, 1628-1650.

361. Mpourmpakis, G.; Tylianakis, E.; Froudakis, G. E., Nano Letters 2007, 7, 1893-1897.

362. Braga, S. F.; Coluci, V. R.; Baughman, R. H.; Galvão, D. S., Chemical Physics Letters 2007, 441, 78-82.

363. Braga, S. F.; Coluci, V. R.; Legoas, S. B.; Giro, R.; Galvão, D. S.; Baughman, R. H., Nano Letters 2004, 4, 881-884.

364. Shi, X.; Pugno, N. M.; Gao, H., Journal of Computational and Theoretical Nanoscience 2010, 7, 517-521.

365. Xie, X.; Ju, L.; Feng, X.; Sun, Y.; Zhou, R.; Liu, K.; Fan, S.; Li, Q.; Jiang, K., Nano Letters 2009, 9, 2565-2570.

366. Viculis, L. M.; Mack, J. J.; Kaner, R. B., Science 2003, 299, 1361.

367. Gao, Y.; Chen, X.; Xu, H.; Zou, Y.; Gu, R.; Xu, M.; Jen, A. K. Y.; Chen, H., Carbon 2010, 48, 4475-4482. 


\section{ANNEX: LIST OF SCIENTIFIC}

\section{Publications and ACHIEVEMENTS}

\section{$\underline{\text { SCIENTIFIC PUbLiCATIONS }}$}

IF $(>5)$

1. Huang, J.; Larisika, M.; Fam, W. H. D.; He, Q.; Nimmo, M. A.; Nowak, C.; Tok, I. Y. A., The extended growth of graphene oxide flakes using ethanol CVD. Nanoscale 2013, 5, 2945-2951.

2. Huang, J.; Fam, D.; He, Q.; Chen, H.; Zhan, D.; Faulkner, S. H.; Nimmo, M. A.; Tok, I. Y. A., The mechanism of graphene oxide as a growth template for complete reduced graphene oxide coverage on an $\mathrm{SiO} 2$ substrate. Journal of Materials Chemistry C 2014, 2, 109-114.

3. Teng, X.; Zhu, Y.; Wei, W.; Wang, S.; Huang, J.; Naccache, R.; Hu, W.; Tok, A. I. Y.; Han, Y.; Zhang, Q.; Fan, Q.; Huang, W.; Capobianco, J. A.; Huang, L., Lanthanide-Doped NaxScF3+x Nanocrystals: Crystal Structure Evolution and Multicolor Tuning. Journal of the American Chemical Society 2012, 134, 8340-8343.

4. Niu, W.; Chen, H.; Chen, R.; Huang, J.; Palaniappan, A.; Sun, H.; Liedberg, B. G. and Tok, A. I. Y.; Synergetically Enhanced Near-Infrared Photoresponse of Reduced Graphene Oxide by Upconversion and Gold Plasmon. Small 2014.

5. Huang, J.; Chen, H.; Niu, W.; Fam, D.; Palaniappan, A.; Larisika, M.; Faulkner, S. H.; Nowak, C.; Liedberg, B.; Nimmo, M. A.; Tok, I. Y. A., Graphene Oxide Biosensors for Interleukin-6 Detection. Biosensors and Bioelectronics 2015. (Submitted)

6. Niu, W.; Chen, H.; Chen, R.; Huang, J.; Palaniappan, A.; Sun, H.; Liedberg, B. G.; Tok, A. I. Y., NaYF4:Yb,Er/MoS2: from synthesis, surface ligands-stripping, to negative infrared photoresponse. Chem Comm 2015. (Accepted)

\section{ARChival PAPERS}

7. Huang, J.; Harvey, J.; Fam, W. H. D.; Nimmo, M. A.; Tok, I. Y. A., Novel Biosensor for InterLeukin-6 Detection. Procedia Engineering 2013, 60, 195-200. 
8. $\quad$ Huang, J.; Harvey, J.; Chen, H.; Fam, W. H. D.; Nimmo, M. A.; Tok, I. Y. A., Complete coverage of reduced graphene oxide on silicon dioxide substrates. Chin. Phys. B, 2014-08-15 2014, 23, 88103-88103.

9. Huang, J.; Harvey, J.; Chen, H.; Nimmo, M. A.; Tok, I. Y. A. Fully Organic Graphene Oxide-based Sensor with Integrated Pump for Sodium Detection, Conference Proceeding of icSports, Vilamoura, Portugal, Vilamoura, Portugal, 2013, 83-88. (Best Student Paper)

10. Huang, J.; Chen, H.; Fam, W. H. D.; Faulkner, S.; Niu, W.; Larisika, M.; Nowak, C.; Nimmo, M. A.; Tok, I. Y. A., Growth of Reduced Graphene Oxide. MRS Proceedings, 2014, 1702.

11. Larisika, M.; Huang, J.; Tok, A.; Knoll, W.; Nowak, C., An improved synthesis route to graphene for molecular sensor applications. Materials Chemistry and Physics 2012, 136, 304-308.

12. Faulkner, S.; Spilsbury, K.; Harvey, J.; Jackson, A.; Huang, J.; Platt, M.; Tok, A.; Nimmo, M., The detection and measurement of interleukin-6 in venous and capillary blood samples, and in sweat collected at rest and during exercise. Eur J Appl Physiol, 2014, 1-10.

13. Chen, H.; Lim, S. K.; Chen, P.; Huang, J.; Wang, Y.; Palaniappan, A.; Platt, M.; Liedberg, B.; Tok, A. I. Y., Reporter-encapsulated liposomes on graphene field effect transistors for signal enhanced detection of physiological enzymes. Physical Chemistry Chemical Physics, 2015, 17 (5), 3451-3456.

14. Huang, J.; Chen, H.; Fam, D.; Faulkner, S. H.; Niu, W.; Larisika, M.; Nowak, C.; Nimmo, M. A.; Tok, I. Y. A., Growth of Reduced Graphene Oxide for Biosensors, Conference proceedings for International Conference in Sports Science and Technology, Advanced Materials for Sports Technology, 2014, 1, 17-23.

15. Chen, H.; Huang, J., Platt, M.; Tok, I. Y., Novel Electronic Biosensors based on Horizontally Aligned Carbon Nanotubes for Applications in Sports Science, Conference proceedings for International Conference in Sports Science and Technology, Advanced Materials for Sports Technology, 2014, 1, 99-105.

16. Huang, J.*; Chen, H.*; M.Platt; M. A.; Tok, I. Y. A., A facile detection route for prostate specific antigen based on horizontally aligned carbon nanotubes, International Conference on Advanced Material and Practical Nanotechnology (ICAMPN), Materials Science Procedia, 2014. (Accepted) 
17. Fam, W. H. D.; Azoubel, S.; Liu, L.; Huang, J.; Mandler, D.; Magdassi, S.; Tok, I. Y. A., Novel Felt Pseudocapacitor based on Carbon Nanotube/Metal Oxides, Journal of Material Science, 2015. (Accepted)

\section{REVIEWS AND CHAPTERS IN BOOKS}

18. Huang, J.; Larisika, M.; Nowak, C.; Tok, I. Y. A., New Methods in Aqueous Graphene (Graphene Oxide) Synthesis for Biosensor Devices. Taylor \& Francis Group: Oxford, UK, 2015; Vol. 1. (Accepted)

19. Huang, J.; Hng, H.H.; Tok, I. Y. A., Nanostructured ZnO Thermoelectric Materials for Microelectronics. Lambert Academic Publishing: Saarbrücken, Germany, 2013.

\section{TECHNOLOGY DisCLOSURES}

20. Huang, J.; Tok, A. I. Y., Facile Low Temperature Reflux Treatment between Pristine CNT and As-synthesized GO to form a new Pristine CNTGO-CNT hybrid structure, 2012 (NTU, TD/094/12)

21. Huang, J.; Tok, A. I. Y.; Nimmo, M.A., Fully Organic Graphene Oxide-based Sensor with Integrated Pump for Sodium Detection, 2013 (LU, D710, 9/9/2013)

\section{HIGHLIGHTED WORK IN MEDIA}

22. Berger, M., We can grow graphene. But graphene oxide. Nanowerk Nanotechnology Spotlight 8/3/2013, 2013. 


\section{PRESENTATIONS AT INTERNATIONAL CONFERENCES AND WORKSHOPS}

1. Report Drafting Committee, ASEAN Committee on Science and Technology, 2008-Current.

2. Huang, J.; Harvey, J.; Hu, C.; Fam, W. H. D.; Nimmo, M. A.; Tok, I. Y. A., Graphene Oxide Growth. Oral Presentation at 13th Joint Symposium on Materials Science and Engineering for the 21st Century, Hsinchu, Taiwan 2013. (Session chair)

3. Huang, J.; Larisika, M.; Chen, H.; Harvey, J.; Fam, D.; Nowak, C.; Nimmo, M. A.; Tok, A., Growth of Graphene Oxide. Oral Presentation at Materials Research Society Spring 2014 Conference, San Francisco, US 2014.

4. Huang, J.; Harvey, J.; Chen, H.; Nimmo, M. A.; Tok, I. Y. A. Fully Organic Graphene Oxide-based Sensor with Integrated Pump for Sodium Detection, Oral Presentation at icSports, Vilamoura, Portugal 2013.

5. Huang, J.; Nimmo, M. A.; Tok, A. I. Y., Post-Treatment of Carbon for Molecular Sensing. Poster presentation at Materials Congress, Carlton House London, UK 2012.

6. Huang, J.; Larisika, M.; Chen, H.; Harvey, J.; Fam, D.; Nowak, C.; Nimmo, M. A.; Tok, A., Growth of Graphene Oxide Using Ethanol CVD. Oral Presentation at 7th International Conference on Materials for Advanced Technologies (ICMAT), Suntec City, Singapore, 2013.

7. Huang, J.; Harvey, J.; Nimmo, M. A.; Tok, I. Y. A., Sensitive Detection of Interleukin-6 using Advanced Carbon-based Transducers. Poster presentation at Small Sciences Symposium, Mandarin Orchard, Singapore 2012.

8. Huang, J.; Harvey, J.; Faulkner, S. H.; Nimmo, M. A.; Tok, I. Y. A., Detection of biomarkers using graphene transducers. Poster Presentation at 4 th M3 Conference, Biopolis, Singapore 2014.

9. Larisika, M.; Huang, J.; Herrera, A.; Nowak, C.; Knoll, W.; Tok, I. Y. A., Ultra-sensitive graphene-based sensor for detection of biomolecules. Poster presentation at International Conference on Materials for Advanced Technologies, Suntec City, Singapore 2011.

10. Huang, J.; Harvey, J.; Faulkner, S.; Nimmo, M. A.; Tok, I. Y. A., Biomarker detection using advanced carbon-based tranducer. Poster presentation at Health and Life Sciences Research Student Conference, Loughborough, UK 2013. 
11. Huang, J.; Harvey, J.; Faulkner, S.; Nimmo, M. A.; Tok, I. Y. A., Reducing the electrical variability in Graphene to enable bio-sensing of Interleukin-6. Poster presentation at School of Sport, Exercise and Health Sciences Post Graduate Research Conference, Loughborough, UK 2013.

12. Huang, J.; Chen, H.; Platt, M; Tok, I. Y. A., A facile detection route for prostate specific antigen based on horizontally aligned carbon nanotubes, International Conference on Advanced Material and Practical Nanotechnology, Jakarta International Expo, Indonesia 2014.

13. Huang, J.; Chen, H.; Faulkner, S. H.; Nimmo, M. A.; Tok, I. Y. A., Enabling the 2-D super-material (Graphene) in Electronics. Poster Presentation at Nanyang Technological University Research Open House, Singapore 2014.

(Awarded Top 5 Technology Poster Prize)

14. Chen, H.; Huang, J.; Fam, W. H. D.; Nimmo, M. A.; Tok, I. Y. A., Coupled-SPR Enhanced Carbon-based Biosensor. Poster presentation at New Diamond and Nano Carbons Conference, Novotel Clarke Quay, Singapore 2013.

\section{ATTENDED INTERNATIONAL CONFERENCES AND WORKSHOPS}

1. Nobel Laureates Panel Discussion at NTU, $7^{\text {th }}$ International Science Youth Forum, NTU, Singapore, 2015.

2. $4^{\text {th }}$ Global Forum on Intellectual Property, Marina Bay Sands, Singapore, 2014.

3. Techventure, Marina Bay Sands, Singapore, 2014.

4. IP Week@ SG, Marina Bay Sands, Singapore, 2014. 


\section{UNIVERSITY TEACHING EXPERIENCE}

\section{Teaching Assistant}

Undergraduate Year 3: Thermodynamics

Undergraduate Year 2: Materials and Design, Mathematics \& Laboratory

Experiments

Post-graduate level: Laboratory Experiments

\section{Supervised}

Final Year Projects (1 year): 6

College Out-reach Projects (1 month each): 4 


\section{RESEARCH AWARDS}

1. Best research blog award, Nanyang Technological University, School of Materials Science and Engineering, 2012.

2. Best student paper award, Oral Presentation at icSports, Vilamoura, Portugal, 2013.

3. Top 10 Finalist poster award, Materials Week, Nanyang Technological University, School of Materials Science and Engineering, 2014.

4. MSE Incentive/Reward Scheme for outstanding performances, Nanyang Technological University, School of Materials Science and Engineering, 2014.

5. Top 5 Cool Tech Research Poster Award, Nanyang Technological University Research Open House, 2014.

\section{UNIVERSITY MODULES}

1. Nanoelectronics and Molecular Electronics, Grade: A+ (5.00)

2. Materials Laboratory Techniques, Grade: A (5.00)

3. Scanning Tunnelling Microscopy and Molecular Self-Assembly, Grade: A (5.00)

4. Advanced Materials, Grade: B (4.00)

5. Materials Characterisation, Grade: $\mathrm{C}+(2.50)$

6. University Teaching for Teaching Assistant

7. Writing Successful Research Grant Proposals

8. Advanced Platforms for Mammalian Cell Bioengineering

9. Working with Media for Research Staff and Postgraduate Researchers

\section{Cumulative GPA: $\underline{4.3 / 5.0}$}

